\title{
Swept-Frequency Sampled Grating Distributed Bragg Reflector Lasers Optimized for Optical Coherence Tomography Applications
}

\author{
A Thesis Presented to the \\ Electrical Engineering Department Faculty of \\ California Polytechnic State University, San Luis Obispo \\ In Partial Fulfillment \\ of the Requirements for the Degree \\ Master of Science Degree in Electrical Engineering
}

by

Brandon J George

December, 2009 
(C) 2009

Brandon J George

ALL RIGHTS RESERVED 


\section{Committee Membership}

Title: Swept-Frequency Sampled Grating Distributed Bragg Reflector Lasers Optimized for Optical Coherence Tomography Applications

Author: Brandon J George

Date Submitted: December, 2009

Committee Chair: Dr. Dennis Derickson

Committee Member: Dr. William L. Ahlgren

Committee Member: Dr. Xiaomin Jin 


\section{Abstract}

Title: Swept-Frequency Sampled Grating Distributed Bragg Reflector Lasers Optimized for Optical Coherence Tomography Applications

Author: Brandon J George

Swept Frequency Source Optical Coherence Tomography (OCT) requires high repetition rate and wide spectral width wavelength tunable sources at a low cost. The sampled grating distributed Bragg reflector (SG-DBR) laser provides wide wavelength tuning range while exhibiting a wavelength switching speed that is among the fastest currently available. The SG-DBR laser is used to generate linear frequency ramps with high repetition rates. Since the SG-DBR laser is currently used for the telecommunications industry in high volume, the price of the system is much lower than current OCT sources. Therefore the SG-DBR laser provides a practical solution for Swept Source OCT.

Four synchronized waveforms sent to the inputs of the laser control the linear frequency ramp. Three of the waveforms control the output frequency, while the fourth waveform controls the output amplifier of the laser to keep the output power stable. Two SG-DBR lasers with overlapping wavelength coverage are also concatenated to increase the bandwidth of the swept frequency source.

The linear ramp stitching points in the frequency ramps are investigated and methods are outlined to reduce them. Finally, experimental OCT tests are performed using the swept frequency sources created to analyze the linearity of our sources. From these test results, an evaluation can be made on the specifications and capabilities of the swept frequency sources and analyze their value for OCT applications. 


\section{Acknowledgments}

I would like to share my extreme gratitude with Dr. Dennis Derickson. Not only has his guidance though my thesis been nothing short of remarkable, but also he has made sure to further my knowledge and my career as well as the project.

Thank you Dr. Xiaomin Jin and Dr. William L. Ahlgren for participating in my thesis committee and for your support in your lectures.

Great appreciation goes to Shane O'Connor and the other students who contributed to the SG-DBR project before me.

Thank you to all the people and organizations that provided funding and made this research possible.

I would like to quickly acknowledge all my friends from Twain Harte, Santa Barbara, New York City, San Luis Obispo, and abroad who keep me sane when the weight of deadlines and new projects bear down on me.

Lastly, I would like to thank my family for their generous and unwavering support of my studies and my career. I certainly would not have made it this far without them. 


\section{Table of Contents}

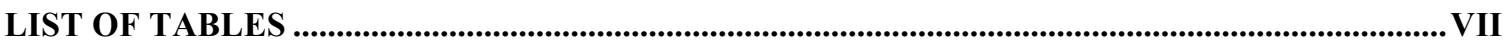

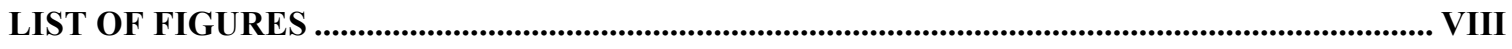

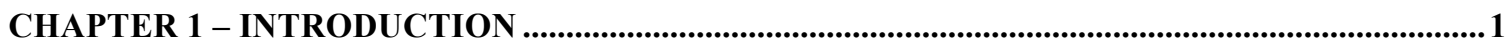

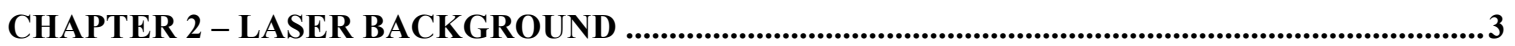

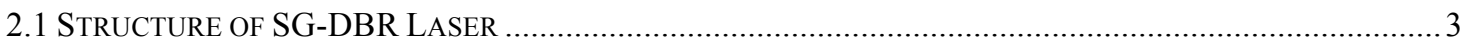

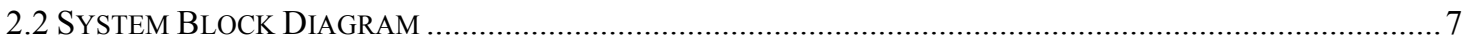

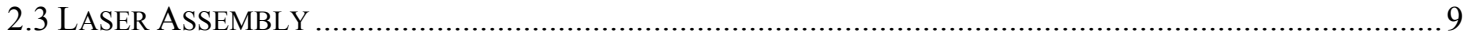

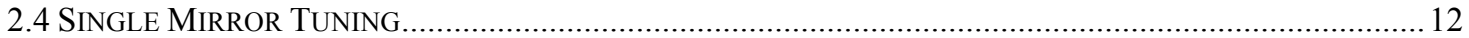

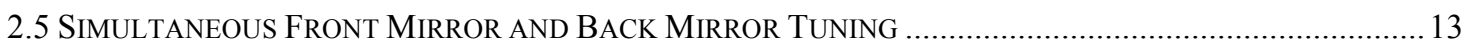

2.6 Simultaneous Front Mirror, BaCK Mirror, AND PHase Tuning ........................................... 14

CHAPTER 3 - OPTICAL COHERENCE TOMOGRAPHY BACKGROUND ...............................17

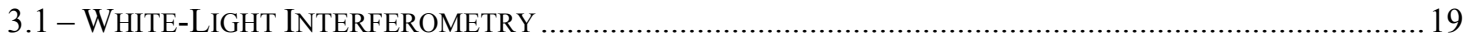

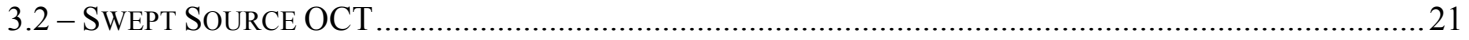

CHAPTER 4 - FREQUENCY CALIBRATION OF A L-BAND AND C-BAND LASER...................26

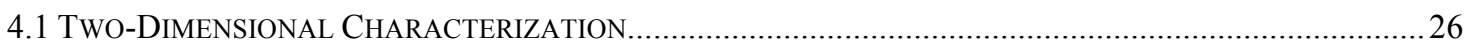

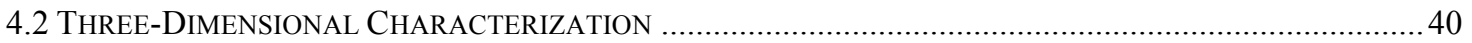

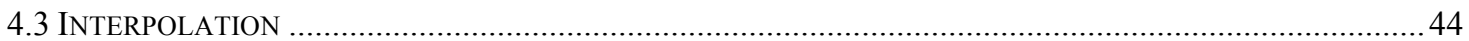

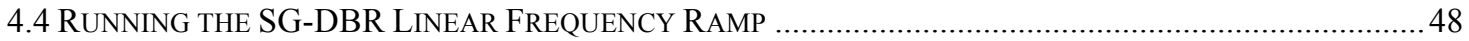

CHAPTER 5 - POWER CALIBRATION AND POWER LEVELING OF A L-BAND AND

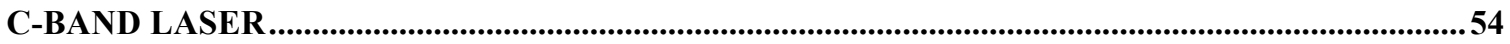

5.1 - CHARACTERIZATION OF THE SEMICONDUCTOR OPTICAL AMPLIFIER............................................5

5.2 - REDUCING THE AMPLITUDE RIPPLE OF THE FREQUENCY RAMP...............................................6 60

CHAPTER 6 - CONCATENATION OF L-BAND AND C-BAND LASERS TO GET A

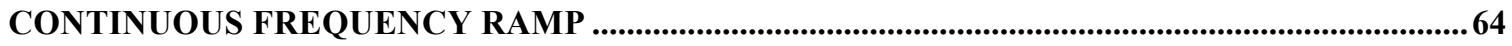

CHAPTER 7 - STITCHING ERRORS AND OCT SYSTEM IMPLICATIONS..............................77

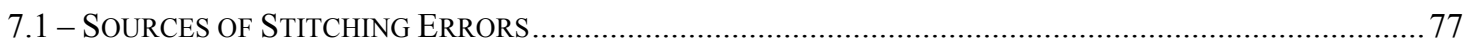

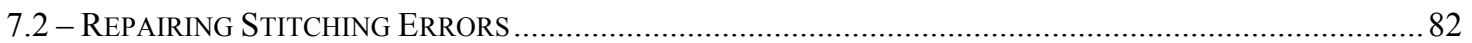

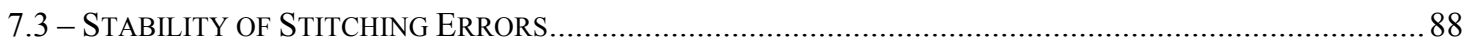

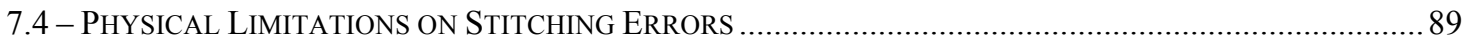

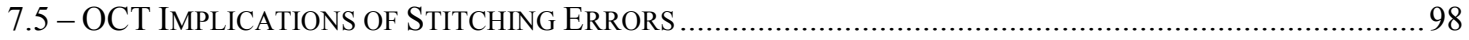

CHAPTER 8 - CONCLUSIONS AND FUTURE WORK............................................................108

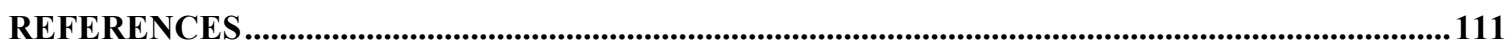

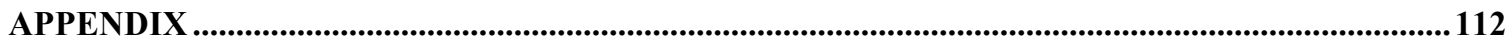




\section{List of Tables}

TABLE 4.1: CHARACTERIZES L-BAND AT THE START AND END POINTS OF THE PATHS ALONG THE FINE. ........33

TABLE 4.2: CHARACTERIZES L-BAND AT THE START AND END POINTS OF THE PATHS ALONG THE NORM......33

TABLE 4.3: CHARACTERIZES C-BAND AT THE START AND END POINTS OF THE PATHS ALONG THE FINE .........33

TABLE 4.4: CHARACTERIZES C-BAND AT THE START AND END POINTS OF THE PATHS ALONG THE NORM...... 34

TABLE 4.5: VALUES OF CURRENT LIMITING RESISTORS USED TO CONVERT CURRENT LIMITS OF PATHS ..........37

TABLE 4.6: CONVERTS TABLE (4.1) TO VOLTAGES FROM CURRENTS BY DIVIDING BY THE...........................38

TABLE 4.7: CONVERTS TABLE (4.2) TO VOLTAGES FROM CURRENTS BY DIVIDING BY THE................................38

TABLE 4.8: CONVERTS TABLE (4.3) TO VOLTAGES FROM CURRENTS BY DIVIDING BY THE..............................38

TABLE 4.9: CONVERTS TABLE (4.4) TO VOLTAGES FROM CURRENTS BY DIVIDING BY THE.............................39

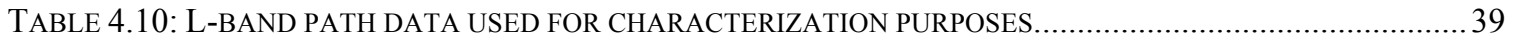

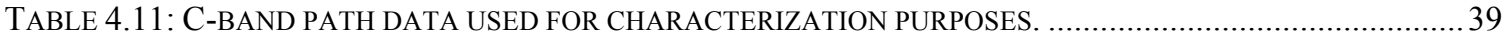

TABLE 5.1: TABLE GIVES VALUES FOR THE AVERAGE VALUES AND STANDARD DEVIATION ..........................62

TABLE 6.1: BIAS POINTS FOR SINGLE FREQUENCY L-BAND AND C-BAND SWITCHING TEST.........................70

TABLE 7.1: L-BAND PORT IMPEDANCE TEST DATA FOR FOUR OF THE PRIMARY SECTIONS IN .........................96

TABLE 7.2: C-BAND PORT IMPEDANCE TEST DATA FOR FOUR OF THE PRIMARY SECTIONS IN........................96

TABLE 7.3: -3 DB BANDWIDTHS FOR THE THREE TRACES SHOWN IN FIGURE (7.21)................................ 100

TABLE 7.4: BEAT SIGNAL SPECTRAL WIDTH DATA FOR THE L-BAND LASER AT 100 Hz AND 10 KHz .......... 106

TABLE 7.5: BEAT SIGNAL SPECTRAL WIDTH DATA FOR THE C-BAND LASER AT 100 Hz AND 10 KHz .......... 106 


\section{List of Figures}

FIGURE 2.1: TOP VIEW OF LASER CHIP SHOWING FIVE PRIMARY SECTIONS OF THE SG-DBR LASER ................4 FIGURE 2.2: SIDE VIEW OF THE CROSS SECTION OF THE SG-DBR LASER ..................................................

FIGURE 2.3: BRAGG REFLECTORS AND THEIR REFLECTIVITY FOR THE FRONT MIRROR AND BACK MIRROR ......6

FIGURE 2.4: LASER OUTPUT WAVELENGTH CONTOUR PLOT DETERMINED BY CURRENTS INTO FRONT..............7

FIGURE 2.5: BLOCK DIAGRAM OF SG-DBR CONTROL. .................................................................

FigurE 2.6: L-BAND LASER ASSEMBLY. FM, BM, AND $\phi$ ARE USED TO CONTROL THE LASER ...................... 10

FIGURE 2.7: C-BAND LASER ASSEMBLY. FM, BM, AND $\phi$ ARE USED TO CONTROL THE LASER ....................... 11

FIGURE 2.8: THE OPTICAL ISOLATOR PREVENTS REFLECTION FROM THE EXTERNAL CIRCUIT FROM ............... 12

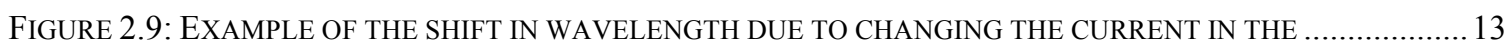

FIGURE 2.10: EXAMPLE OF THE SHIFT IN WAVELENGTH DUE TO CHANGING THE CURRENT IN THE ................14

FIGURE 2.11: DECREASING THE CURRENT INTO THE PHASE SECTION RELATES TO AN INCREASE OF .............. 15

FIGURE 3.1: ONE-DIMENSIONAL OCT TEST EXAMPLE SHOWING HOW REFLECTED SIGNALS GIVE ................. 18

FIGURE 3.2: SAMPLE TWO-DIMENSIONAL OCT IMAGE OF THE SKIN OF A HUMAN FINGER............................ 19

FIGURE 3.3: WHITE-LIGHT INTERFEROMETRY TEST SETUP BLOCK DIAGRAM............................................ 19

FIGURE 3.4: RESULTS OF A WHITE-LIGHT INTERFEROMETRY OCT TEST SHOWING THE MANY ........................20

FIGURE 3.5: SWEPT SOURCE OCT TEST SETUP. MOVABLE MIRROR, SHOWN IN FigURE (3.3) .......................22

FIGURE 3.6: COMBINED SIGNALS FROM THE STATIONARY MIRROR AND THE DUT. $\Delta$ T IS THE TIME .............23

FIGURE 4.1: BLOCK DIAGRAM OF THE TWO-DIMENSIONAL LASER CHARACTERIZATION SETUP .....................227

FIGURE 4.2: THREE-DIMENSIONAL (MODE MAP) PLOT OF WAVELENGTH VS. MIRROR CURRENTS ...................28

FIGURE 4.3: THREE-DIMENSIONAL (MODE MAP) PLOT OF WAVELENGTH VS. MIRROR CURRENTS ..................29

FIGURE 4.4: CONTOUR PLOT FOR L-BAND LASER SHOWING FINE RESOLUTION CHARACTERIZATION ...............30

FIGURE 4.5: CONTOUR PLOT FOR L-BAND LASER SHOWING NORMAL RESOLUTION CHARACTERIZATION ........30

FIGURE 4.6: CONTOUR PLOT FOR C-BAND LASER SHOWING FINE RESOLUTION CHARACTERIZATION ..............31

FIGURE 4.7: CONTOUR PLOT FOR C-BAND LASER SHOWING NORMAL RESOLUTION CHARACTERIZATION ........ 31

FIGURE 4.8: L-BAND LASER PLOT SHOWING THE WAVELENGTH VERSUS CURRENT CURVES ..........................32

FIGURE 4.9: BLOCK DIAGRAM OF SG-DBR CONTROL USING VOLTAGE SOURCES AND CURRENT ...................36

FIGURE 4.10: BLOCK DIAGRAM OF THREE-DIMENSIONAL CHARACTERIZATION TEST SETUP ...........................41

FIGURE 4.11: TWO SEPARATE BIAS POINTS FOR THE SAME CHARACTERIZATION POINT .................................42

FIGURE 4.12: DEMONSTRATION OF TWO TYPES OF STITCHING ERRORS. THE RED FREQUENCY RAMP ............ 43

FIGURE 4.13: DIFFERENCES DUE TO A LINEAR FREQUENCY RAMP AND A LINEAR WAVELENGTH RAMP .......... 46

FIGURE 4.14: INPUT ARB WAVEFORMS THAT ARE USED TO CONTROL THE LINEAR FREQUENCY RAMPS ........48

FIGURE 4.15: BLOCK DIAGRAM OF THE TEST SETUP TO RUN A LINEAR FREQUENCY RAMP .........................49

FIGURE 4.16: CURRENT LIMITING CIRCUIT USED IN LASER TEST SETUPS TO PREVENT OVERLOADING ............50

FIGURE 4.17: HIGH POWER RESISTOR PROTECTS THE LASER SECTION FROM EXCEEDING THE MAXIMUM .......50

FIGURE 4.18: TEST SETUPS FOR THE L-BAND AND C-BAND LASER. THE TEST SETUP ON THE LEFT .................51

FIGURE 4.19: FREQUENCY RAMPS OF L-BAND AND C-BAND LASERS RUN AT A 1 HZ REPETITION ..................53

FIGURE 5.1: LASER OUTPUT POWER DETERMINED FROM THREE-DIMENSIONAL CHARACTERIZATION.............55

FIGURE 5.2: SOA CHARACTERIZATION CURVES FOR L-BAND LASER. LEGEND REFERS TO THE ....................56

FIGURE 5.3: SOA CHARACTERIZATION CURVES FOR C-BAND LASER. LEGEND REFERS TO THE ....................57

FIGURE 5.4: SHIFTED SOA CHARACTERIZATION CURVES FOR L-BAND LASER. LEGEND REFERS ..................58

FIGURE 5.5: SHIFTED SOA CHARACTERIZATION CURVES FOR C-BAND LASER. LEGEND REFERS ...................59

FIGURE 5.6: CURVES DEMONSTRATE THE WAVELENGTH DEPENDENCE OF THE SOA LASER OUTPUT .............60

FIGURE 5.7: TEST SETUP USED FOR A POWER LEVELED LINEAR FREQUENCY RAMP. ANOTHER ARB .............61

FIGURE 5.8: POWER LEVELED FREQUENCY RAMPS OF L-BAND AND C-BAND LASERS RUN AT A $1 \mathrm{~Hz}$............62

FIGURE 6.1: CONCATENATION OF L-BAND AND C-BAND LASERS USING A -3 DB COUPLER. ........................64

FIGURE 6.2: TEST SETUP USED TO FIND THE ATTENUATION OF THE SOA VERSUS WAVELENGTH ..................65

FIGURE 6.3: SOA ATTENUATION VERSUS WAVELENGTH MEASUREMENT DUE TO L-BAND ARB SOA ...........66

FIGURE 6.4: SOA ATTENUATION VERSUS WAVELENGTH MEASUREMENT DUE TO C-BAND ARB SOA ...........66

FIGURE 6.5: TEST SETUP USED TO ANALYZE THE LASER OUTPUT, IN FIGURE (6.6) WHEN THE ARB ..............67

FIGURE 6.6: FIGURE SHOWS THE ATTENUATION OF THE LASER OUTPUT DUE TO TURNING OFF THE SOA .......68

FIGURE 6.7: SHOWS C-BAND SOA RISE WAVEFORM, L-BAND SOA FALL WAVEFORM, AND THE ARB ..........69

FIGURE 6.8: TEST SETUP USED FOR SWITCHING BETWEEN THE TWO LASERS AT A SINGLE FREQUENCY.........70

FIGURE 6.9: ZOOMED IN PLOT OF SWITCHING POINT BETWEEN L-BAND AND C-BAND LASERS ......................71 
FIGURE 6.10: TEST SETUP FOR THE CONCATENATION OF TWO POWER LEVELED LINEAR FREQUENCY ............72

FIGURE 6.11: ARB TUNING WAVEFORMS NEEDED FOR CREATING A SINGLE POWER LEVELED ......................74

FIGURE 6.12: CONCATENATED POWER LEVELED LINEAR FREQUENCY RAMPS FROM AN L-BAND AND ............75

FIGURE 7.1: IMAGE SHOWS AN EXAMPLE OF THE 8 TUNING PATHS STITCHED TOGETHER TO CREATE .............78

FIGURE 7.2: DEMONSTRATION OF TWO TYPES OF STITCHING ERRORS. THE RED FREQUENCY RAMP ..............79

FIGURE 7.3: BLOCK DIAGRAM OF THE TEST SETUP NEEDED TO ANALYZE THE LINEARITY OF .......................8

FIGURE 7.4: OSCILLOSCOPE OUTPUT FROM 100 PM INTERFEROMETER SHOWING THE FIRST ..........................81

FIGURE 7.5: ZOOMED IN PLOT OF OSCILLOSCOPE OUTPUT FROM 100 PM INTERFEROMETER ..........................81

FIGURE 7.6: FIRST STITCH POINT FIXED FOR THE L-BAND LASER TRACE SHOWN IN FIGURE (7.4) .................82

FIGURE 7.7: ZOOMED IN PLOT OF FIRST STITCH POINT FIXED FROM THE L-BAND TRACE SHOWN...................83

FIGURE 7.8: OSCILLOSCOPE DATA FOR THE FIRST STITCHING ERROR IN THE L-BAND LASER AT ....................84

FIGURE 7.9: DEMONSTRATION OF DELAYING THE FREQUENCY RAMP TO WAIT APPROXIMATELY ...................85

FIGURE 7.10: PLOTS OF PHASE, FRONT MIRROR, BACK MIRROR, AND SOA WAVEFORMS SHOWING ...............85

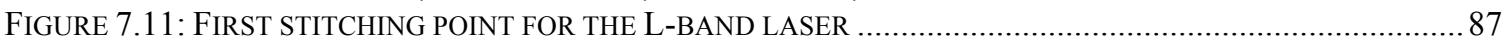

FIGURE 7.12: ZOOMED IN PLOT OF FIRST STITCHING POINT FIXED AT A 10 KHZ REPETITION RATE.................87

FIGURE 7.13: STABILITY TEST FOR THE FIRST STITCHING ERROR OF THE L-BAND POWER LEVELED ...............89

FIGURE 7.14: RLC MODEL USED FOR EACH SECTION ON THE SG-DBR LASER. .........................................90

FIGURE 7.15: PORT IMPEDANCE TEST SETUP. VNA BIAS-TEE CONNECTION AND A CURRENT ........................91

FIGURE 7.16: SWEPT FREQUENCY PORT IMPEDANCE PLOT FOR L-BAND PHASE SECTION BIASED ON .............92

FIGURE 7.17: SWEPT FREQUENCY PORT IMPEDANCE PLOT FOR L-BAND PHASE SECTION BIASED OFF ...........93

FIGURE 7.18: VNA TDR MEASUREMENT DATA FOR L-BAND PHASE SECTION BIASED “ON.” .........................94

FIGURE 7.19: VNA TDR MEASUREMENT DATA FOR L-BAND PHASE SECTION BIASED “OFF.”......................99

FIGURE 7.14: RLC MODEL CIRCUIT SHOWN AGAIN FOR QUICK REFERENCE FOR TABLE (7.1)......................96

FIGURE 7.20: VISUAL REPRESENTATION OF WAVELENGTH TUNING VERSUS TIME .......................................97

FIGURE 7.21: COMBINED SIGNALS FROM THE STATIONARY MIRROR AND THE DUT...................................98

FIGURE 7.22: ORIGINAL TRACE (TOP), “FIXED” TRACE (MIDDLE), AND QUANTIZED SINUSOID....................100

FIGURE 7.23: TEST SETUP USED FOR OCT TESTING OF THE L-BAND AND C-BAND FREQUENCY RAMPS. ...... 101

FIGURE 7.24: SPECTRUM ANALYZER OUTPUT RESULTING FROM A 3 MM DELAY SETTING ON THE 8504B..... 102

FIGURE 7.25: COMBINED OCT MEASUREMENTS SHOWING THE BEAT SIGNALS FOR SELECTED .................... 103

FIGURE 7.26: OCT PEAK POWER DATA FOR L-BAND LASER AT 100 HZ REPETITION RATE FOR.................... 104

FIGURE 7.27: OCT PEAK POWER DATA FOR C-BAND LASER AT 100 HZ REPETITION RATE FOR ................... 104

FIGURE 7.28: OCT PEAK POWER DATA FOR L-BAND LASER AT 10 KHZ REPETITION RATE FOR. .................. 105

FIGURE 7.29: OCT PEAK POWER DATA FOR C-BAND LASER AT 10 KHZ REPETITION RATE FOR................... 105 


\section{CHAPTER 1 - Introduction}

Optical sensing techniques such as Light Detection and Ranging (LIDAR) and Swept Frequency Source Optical Coherence Tomography (OCT) require new high sweep repetition rate and wide spectral width optical sources with low cost. Sampled grating distributed Bragg reflector (SG-DBR) lasers fulfill these requirements. The SG-DBR laser is one of the fastest wavelength switching lasers of its class (low power wavelength tunable semiconductor lasers) and has a wide wavelength tuning range of approximately 50 nanometers $(\mathrm{nm})$.

Chapter 2 of this thesis delves into structure and specifications of the SG-DBR laser. A quick introduction into the processes of controlling a SG-DBR is then shown. Finally, the tuning characteristics of the SG-DBR laser are presented, which will outline some of the positive and negative attributes of the laser.

A background of Optical Coherence Tomography measurements is established in Chapter 3. OCT can produce high resolution three-dimensional images of objects when set at a close distance to the optical source. These three-dimensional images are extremely useful in medical applications. Advantages of Swept Frequency Source Interferometry over White-Light Interferometry OCT measurements will be explained.

In Chapter 4, a method for producing a linear frequency ramp over then entire wavelength tuning range of the laser is created. The first step, in Section 4.1 creates a laser mode map of the full laser wavelength tuning range. In Section 4.2 optimization of the laser output occurs for a set number of points, which span the tuning range of the laser. Section 4.3 linearly interpolates the optimized points giving bias waveforms that will create a continuous frequency ramp spanning the tuning range of the laser. The main 
contribution from this chapter is the linear frequency interpolation since previous research focused on linear wavelength interpolation.

All further material presented in Chapters 5 - 7 can be attributed entirely to this thesis, unless cited. A negative attribute of the SG-DBR laser stems from that the output power is somewhat dependant to the currents applied to the frequency bias ports.

Chapter 5 creates a procedure for reducing the variance of the output power as the output frequency of the laser is changed.

Large wavelength tuning range optical sources are desirable for OCT applications, since time resolution is directly proportional to the frequency scan range. Chapter 6 attempts to concatenate two separate SG-DBR lasers with overlapping wavelength coverage to approximately double the bandwidth of the swept frequency source.

Finally, Chapter 7 investigates some of the faults of the linear frequency ramps, presents method for dealing with or removing the errors, and discovers how detrimental they are for an OCT measurement. 


\section{CHAPTER 2 - Laser Background}

The sampled grating distributed Bragg reflector InGaAs/InP diode laser is a fast tuning, wide wavelength tuning range solution for many applications. It can be configured to cover approximately 50 nanometer $(\mathrm{nm})$ range at various wavelengths in the infrared band.

The laser consists of five sections, each with its own input. Two sections control the output power of the laser. The gain section provides the cavity of the laser with the population inversion needed for laser gain [4]. The semiconductor optical amplifier (SOA) section provides gain to boost the laser output to more than $10 \mathrm{dBm}$ [3]. The SOA section can be used to modulate the output power as desired and is also used as a switch to shutter the output light with more than 50 decibels $(\mathrm{dB})$ of attenuation. Three sections of the SG-DBR laser control the output wavelength. Front mirror (FM) and back mirror (BM) sections are sampled grating distributed Bragg reflectors (SG-DBR). The phase section of the laser adjusts the effective "length" of the cavity therefore continually adjusting the lasing wavelength by small amounts.

\subsection{Structure of SG-DBR Laser}

Figure (2.1) and Figure (2.2) show the structure of the SG-DBR laser. A SGDBR laser has the capability of tuning wavelengths quickly, approximately 5 nanoseconds (ns), over its entire $50 \mathrm{~nm}$ bandwidth [11]. The front mirror (FM), back mirror (BM), and phase $(\phi)$ sections control the output frequency of the SG-DBR laser and utilize high bandgap materials to reduce optical loss. The gain and the semiconductor optical amplifier (SOA) sections control the output power and use low bandgap materials to provide gain [1]. 


\section{Back Gain Phase Front Output Mirror Segment Adjust Mirror Amplifier}

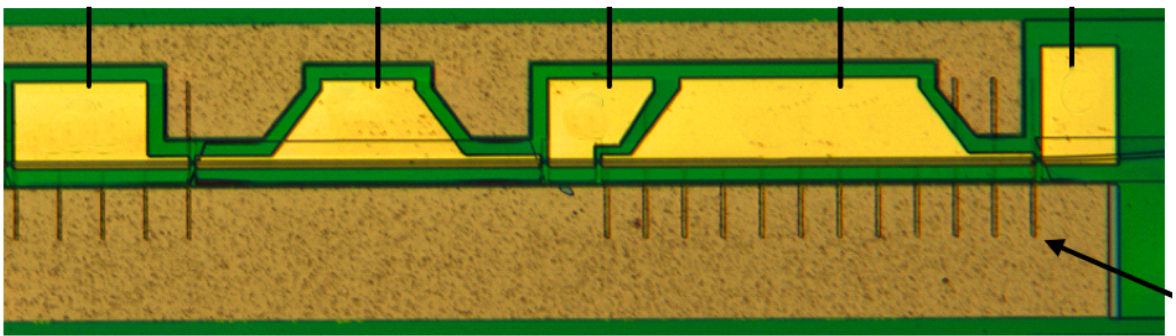

Bragg Grating periodically removed etch lines

Figure 2.1: Top view of laser chip showing five primary sections of the SG-DBR laser [1].

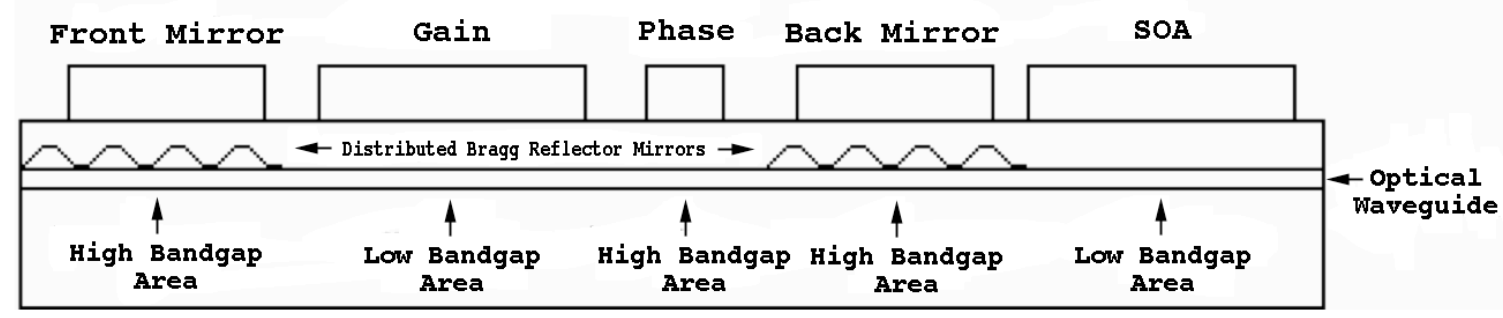

Figure 2.2: Side view of the cross section of the SG-DBR laser [1].

The front mirror and back mirror sections provide the reflectors for the laser cavity. Both mirrors are Bragg reflectors with the Bragg grating created by etching interruptions in the Bragg grating in the laser. The front mirror and back mirror sections give wide frequency tuning ranges from $1525 \mathrm{~nm}$ to $1575 \mathrm{~nm}$ for a C-band laser and 1575 $\mathrm{nm}$ to $1625 \mathrm{~nm}$ for a L-band laser, however the tuning is not continuous [1].

The phase section provides for continuous fine tuning of the output frequency by modulating the effective length of the laser cavity. The current applied to the phase section controls the delay of the laser path and therefore controls the longitudinal mode spacing since the laser must have an integer number of $2 \pi$ radians phase shift around the laser cavity [1][4]. The phase section can continuously vary the output wavelength approximately $1 \mathrm{~nm}[2]$. 
The gain section provides the stimulated emission and optical gain needed for laser operation [4]. The SOA provides further amplification of the laser signal outside the laser cavity allowing greater than 10 milliwatts $(\mathrm{mW})$ of output power as well as providing a method of leveling the output power of the laser with respect to frequency $[3]$.

The frequency at which a laser operates at is determined by two factors. First since a laser needs positive feedback to operate, a laser only can lase in discrete longitudinal modes according to Equation 2.1 (where $f$ is frequency, $\mathrm{m}$ is an integer, $\mathrm{c}$ is the speed of light in a vacuum, $n$ is the refractive index of the medium).

$$
f=\frac{m c}{2 n L}
$$

Next, one of these modes needs a round trip gain greater than 1 to create a laser (usually a dominant mode) [4]. The front mirror, back mirror and phase sections all play a part in the round trip phase. The phase section provides a "fine tune" for the round trip phase condition by continuously shifting the longitudinal modes.

The periodic interruptions in the Bragg gratings of the front mirror and the back mirror have slightly different spacings, which produces slightly different reflectivity versus wavelength profiles, since the reflectivity of a Bragg reflector is dependant on the periodic spacing. Since there is a different periodicity in the reflectivity versus wavelength for the front mirror and back mirror, only one alignment of the front mirror and back mirror peaks will occur. Figure (2.3) illustrates the Bragg reflectors and their reflectivity profiles [1]. 
Back Mirror
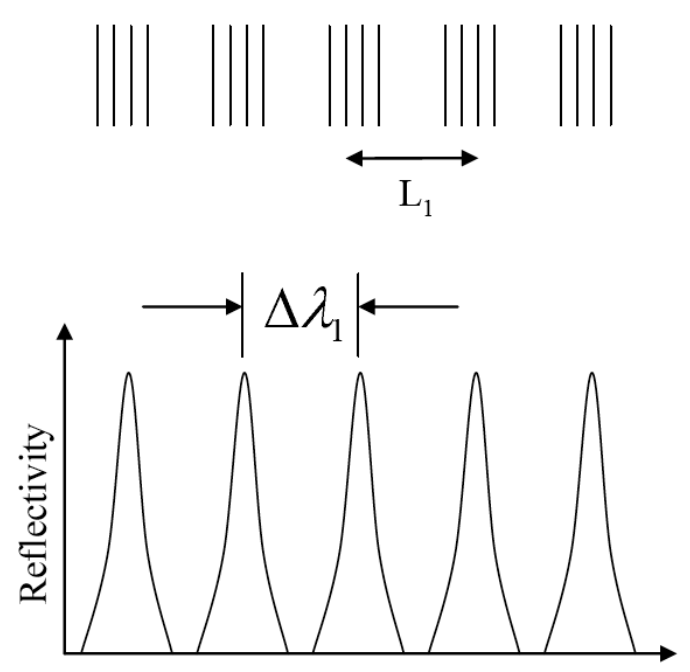

Wavelength
Front Mirror
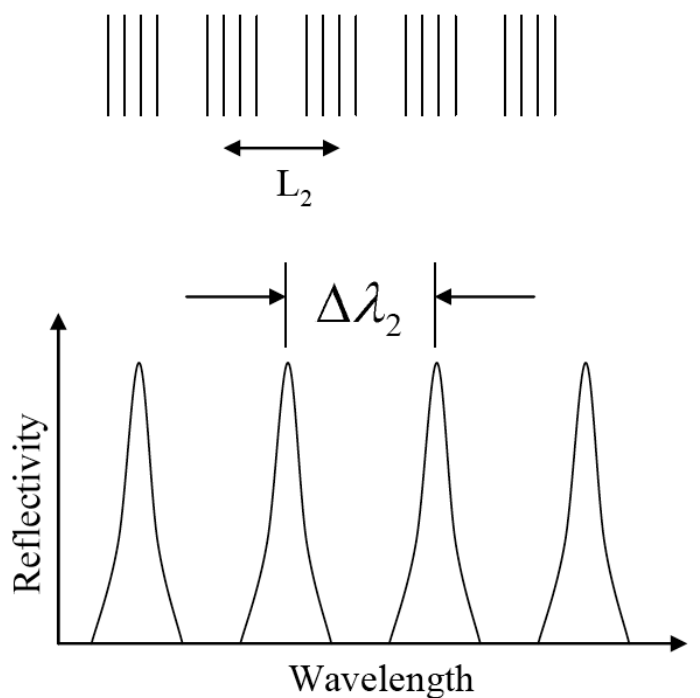

Figure 2.3: Bragg reflectors and their reflectivity for the front mirror and back mirror. The periodic interruptions in the Bragg grating cause a periodic peaks in reflectivity versus wavelength. The lengths between the gratings are different in the front mirror and back mirror to assure only one set of peaks occur at the same wavelength [2].

The reflectivity peak spacing is determined by the following [1].

$$
\Delta \lambda=\frac{\lambda^{2}}{2 n L}
$$

With the spacing between Bragg segments equal to approximately 72 microns $(\mu \mathrm{m})$, this relates to approximately $5 \mathrm{~nm}$ between reflectivity peaks. Applying current to the front mirror and back mirror sections will change the index of refraction in the segments, which will shift the reflectivity peaks and change the wavelength at which two peaks overlap [1]. Figure (2.4) shows the wavelengths of the laser output depending on the currents into the front mirror and back mirror. 


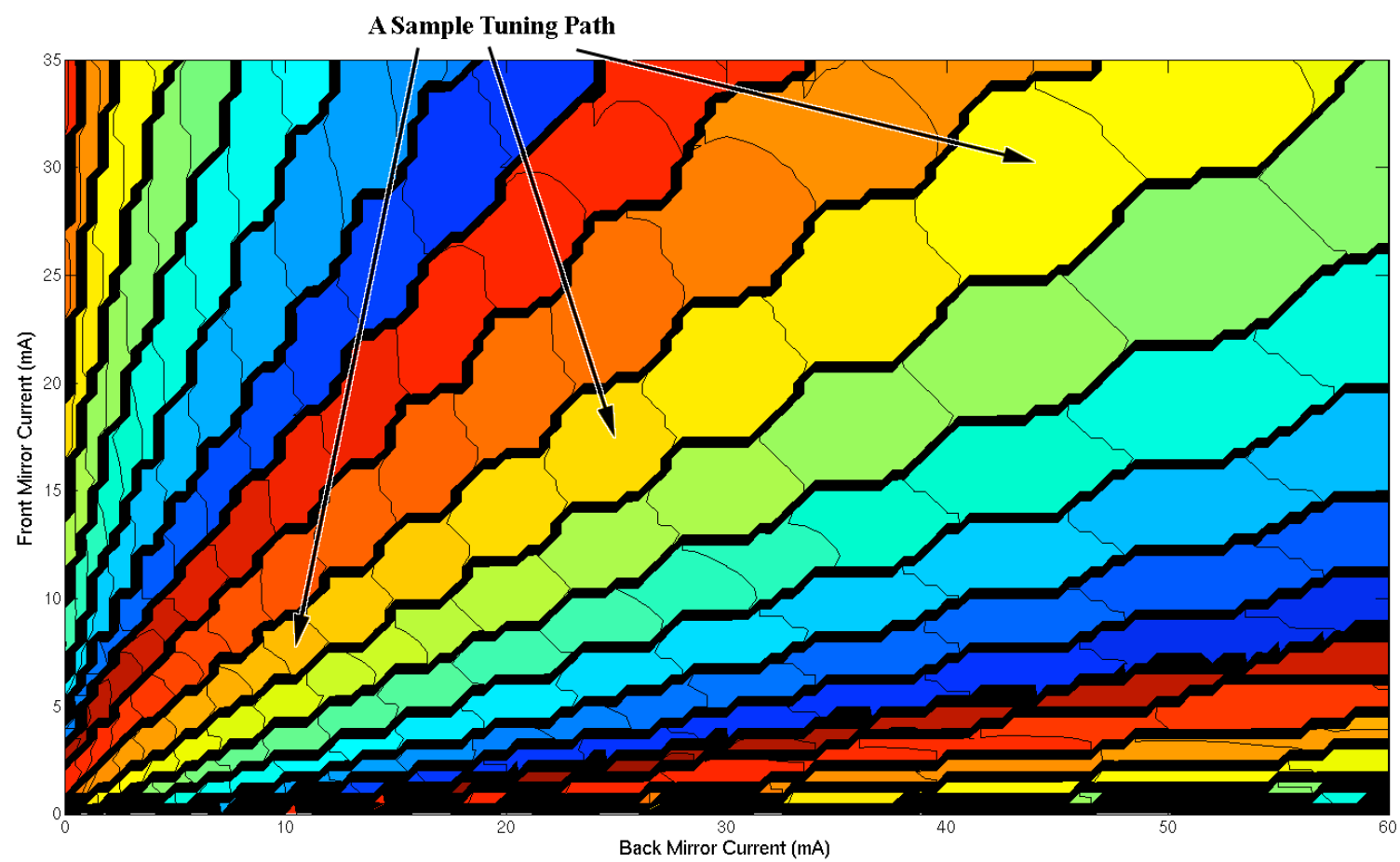

Figure 2.4: Laser output wavelength contour plot determined by currents into front mirror and back mirror with the color in the plot representing wavelength (red higher wavelengths, blue lower wavelengths). Semi-continuous tuning with mode hops of $0.3 \mathrm{~nm}$ can be attained by following the paths of like color.

\subsection{System Block Diagram}

To control the five segments of the SG-DBR laser as well as analyze the output frequency and power, the laser is connected as shown in Figure (2.5). 


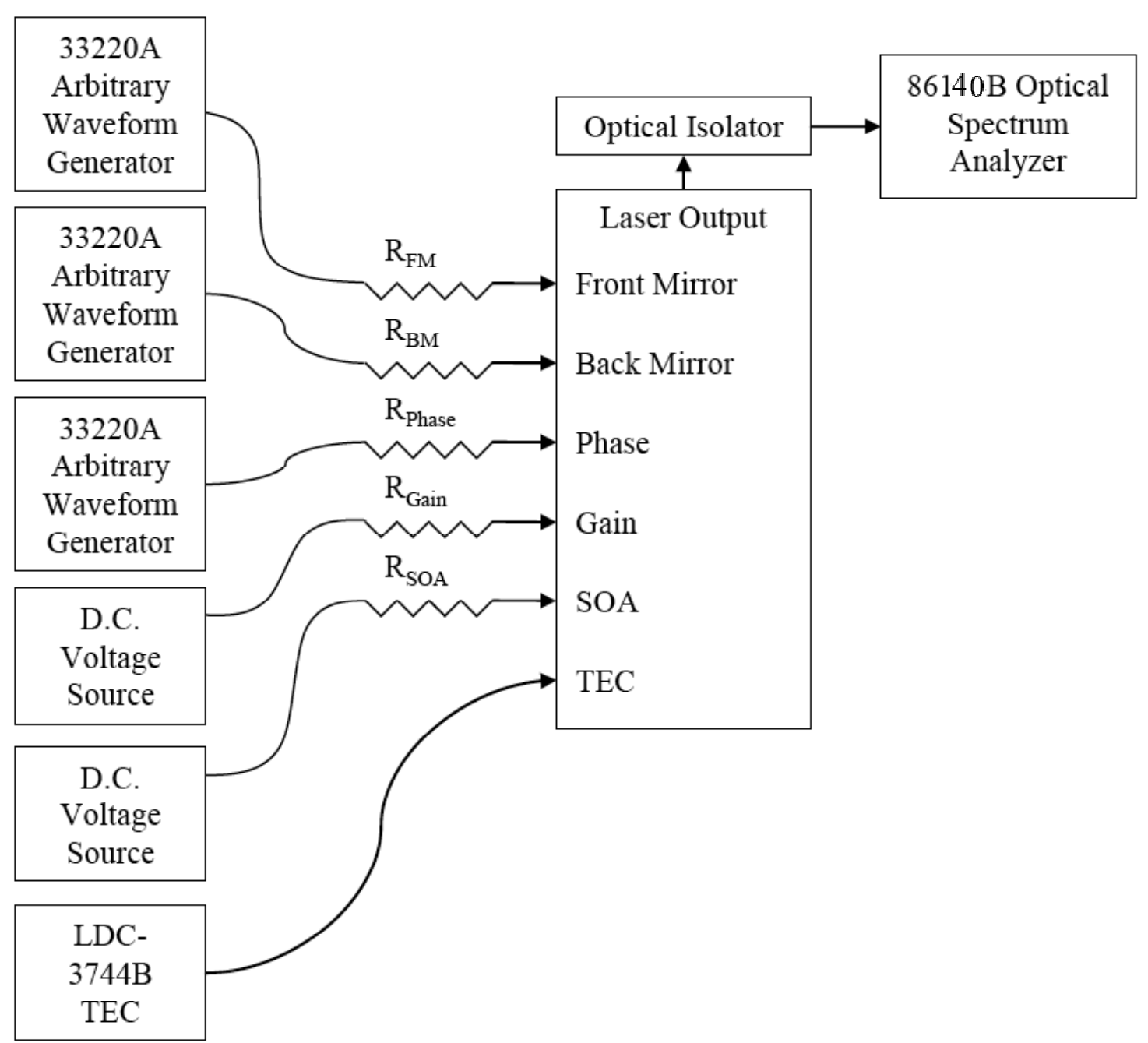

Figure 2.5: Block diagram of SG-DBR control. Three Arbitrary Waveform Generators (ARBs) are used with current limiting resistors to control the front mirror, back mirror, and phase sections. Gain and SOA sections, also with current limiting resistors, are powered by D.C. voltage sources. The SG-DBR laser is actively cooled by a thermoelectric cooler (TEC). An optical isolator is used to attenuate reflected light.

The front mirror, back mirror and phase sections are controlled with Agilent 33220A Arbitrary Waveform Generators (ARBs) that can be used as DC voltage sources as well. The gain and SOA sections are controlled with a D.C. voltage source. The resistors are used to limit the current into each section as a precaution to prevent damage to the laser. A thermoelectric cooler (TEC) and a thermister temperature sensor are required to keep the SG-DBR laser at $20{ }^{\circ} \mathrm{C}$. An optical isolator is needed at the output 
of the laser to reduce the reflected signal into the output of the laser. A $30 \mathrm{~dB}$ isolator is included inside the laser package, but this is generally not sufficient, so an extra $60 \mathrm{~dB}$ isolator was added outside the laser. An Agilent 86140B Optical Spectrum Analyzer records the output frequency and power information.

\subsection{Laser Assembly}

The SG-DBR Widely Tunable Laser Diode Module is mounted in a 24-pin butterfly package. The laser is packaged without filtering capacitors on each of the inputs for this project, since in telecommunications applications these capacitors are used to reduce current noise to the wavelength control sections of the laser [3]. To allow high frequency drive currents in the laser, stray capacitances and inductances need to be minimized. Semi-rigid coaxial cables are soldered to the pins, connecting the laser to the ARB or D.C. voltage source [2]. These cables allow for a $50 \Omega$ environment to extend to the pins of the laser package. To increase the capacitance to ground of the pins, which will bring the pins closer to a $50 \Omega$, a square piece of RT/Duroid is mounted between the

pins and the ground plane [2]. Figure (2.6) and Figure (2.7) show the completed L-band and C-band laser assemblies. 


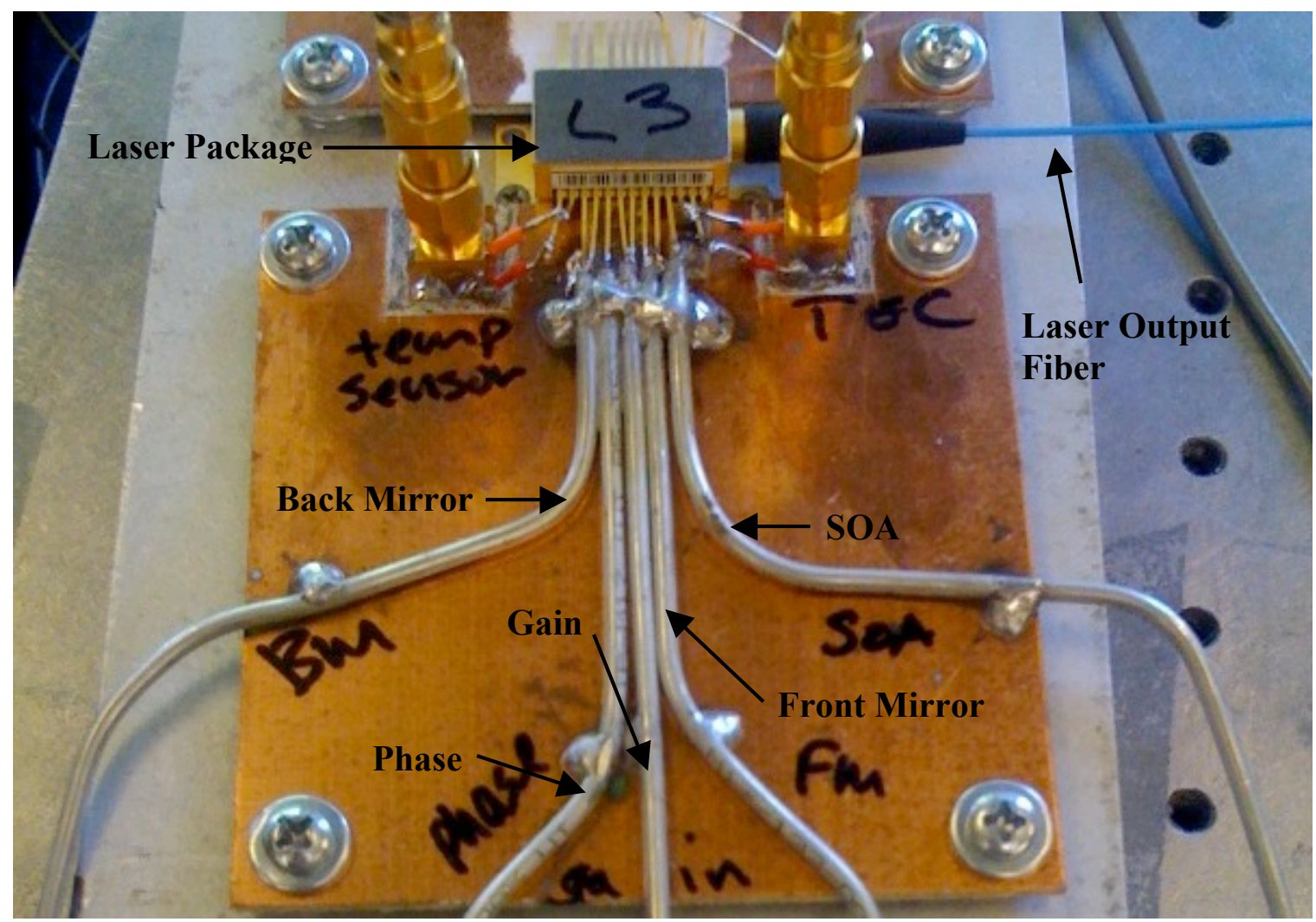

Figure 2.6: L-band Laser Assembly. FM, BM, and $\phi$ are used to control the laser wavelength. The Gain and SOA are used to control the output power. The temperature sensor monitors the temperature inside the laser and the TEC provides cooling to keep the laser at $20^{\circ} \mathrm{C}$. A fiber pigtail provides the laser output. The laser is mounted on copper for its low heat and electrical resistance. 


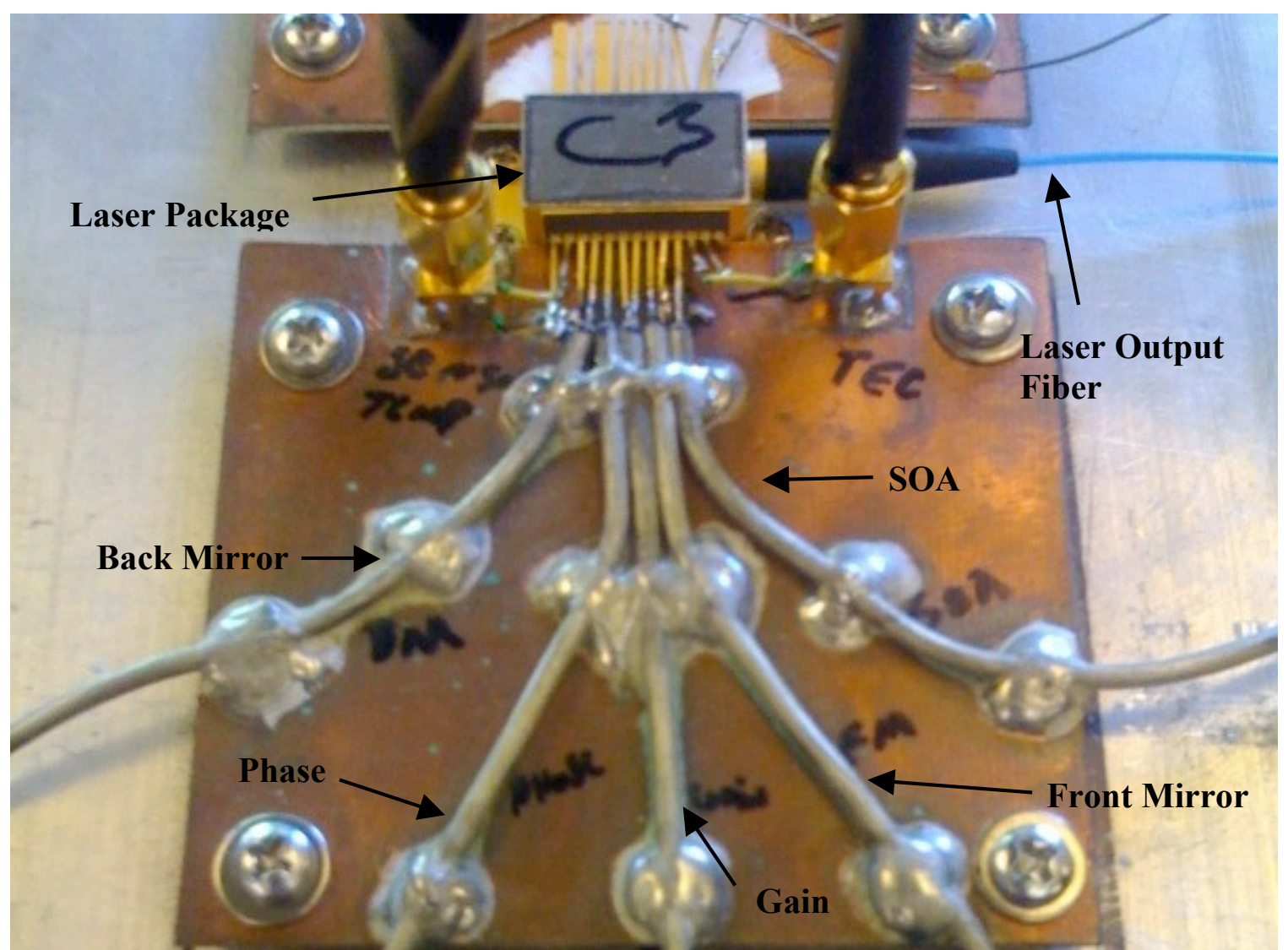

Figure 2.7: C-band Laser Assembly. FM, BM, and $\phi$ are used to control the laser wavelength. The Gain and SOA are used to control the output power. The temperature sensor monitors the temperature inside the laser and the TEC provides cooling to keep the laser at $20^{\circ} \mathrm{C}$. A fiber pigtail provides the laser output. The laser is mounted on copper for its low heat and electrical resistance.

The laser also needs to be mounted to a heat sink and cooled to $20{ }^{\circ} \mathrm{C}$. A Thermoelectric cooler (TEC) is connected as shown in Figure (2.5) to keep the laser at $20{ }^{\circ} \mathrm{C}$. The TEC measures the temperature of the laser from an analog feed back loop provided by the thermistor output and then adjusts the current into the temperature control port of the laser. The TEC inputs and outputs do not require low capacitance or inductance since the TEC does not use high frequency currents [2]. The TEC is always used while the SG-DBR laser is on, therefore the TEC may be left out of block diagrams from this point on. 
The laser provides the optical output coupled directly into a fiber pigtail. The laser package includes a built in isolator, however, an additional external isolator is needed before the laser output can be utilized. Figure (2.8) shows the optical isolator used to increase stability in the laser by blocking reflections the external system might introduce. The optical isolator has very little loss and is always connected to the output of the laser and so the output of the isolator will be considered the output of the laser [2].

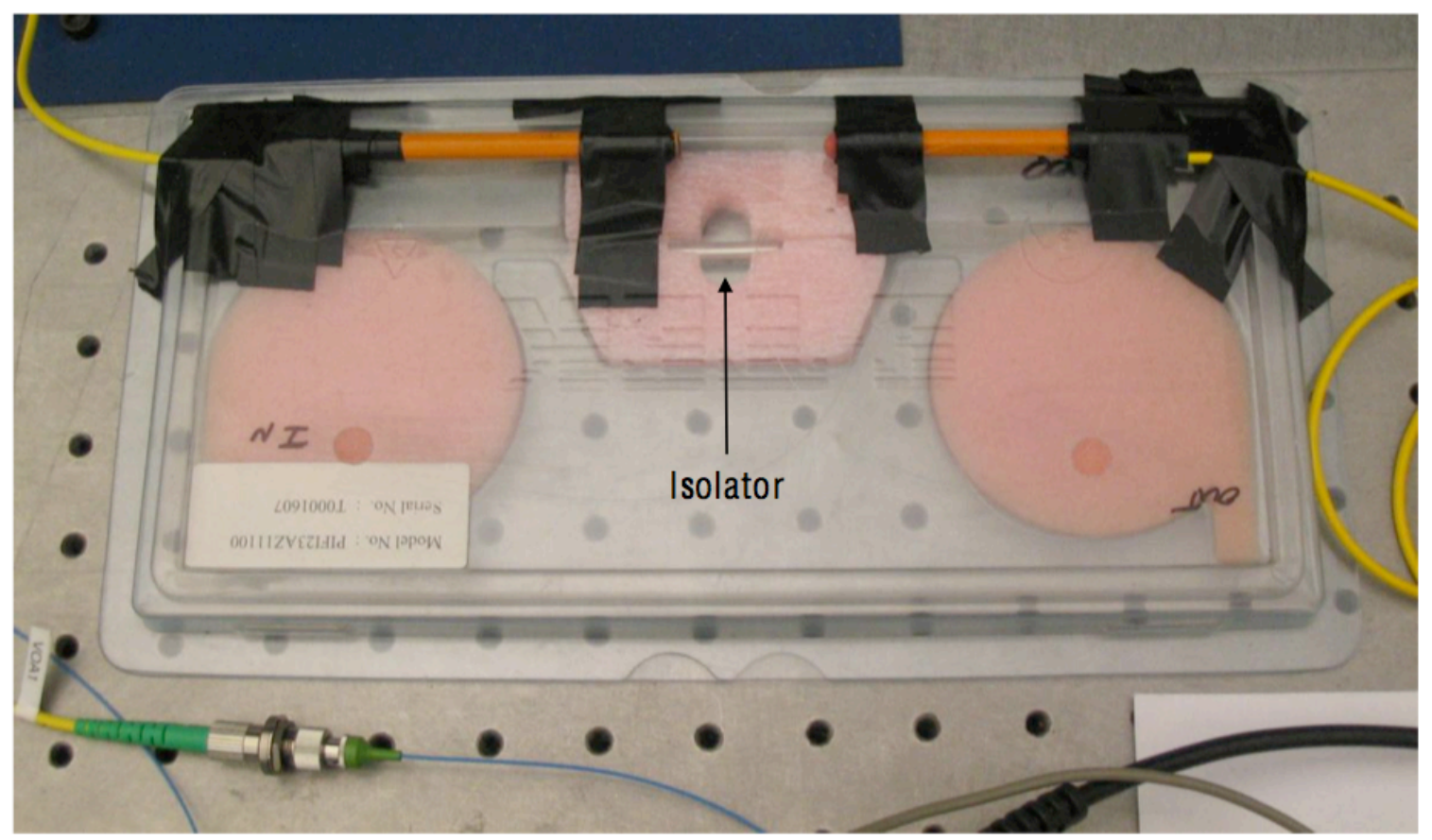

Figure 2.8: The optical isolator prevents reflection from the external circuit from returning to the laser [2].

\subsection{Single Mirror Tuning}

Each primary section in the SG-DBR consists of a PIN diode [3]. The refractive index in the front mirror and back mirror is nominally approximately 3.3. Applying current only to the front mirror or back mirror will change the index of refraction in the section and that in turn will cause a shift in the reflectivity peaks versus wavelength. As 
the reflectivity peaks shift, the aligned peaks will jump modes in approximately $5 \mathrm{~nm}$ steps [1]. Figure (2.9) visually represents single mirror tuning.
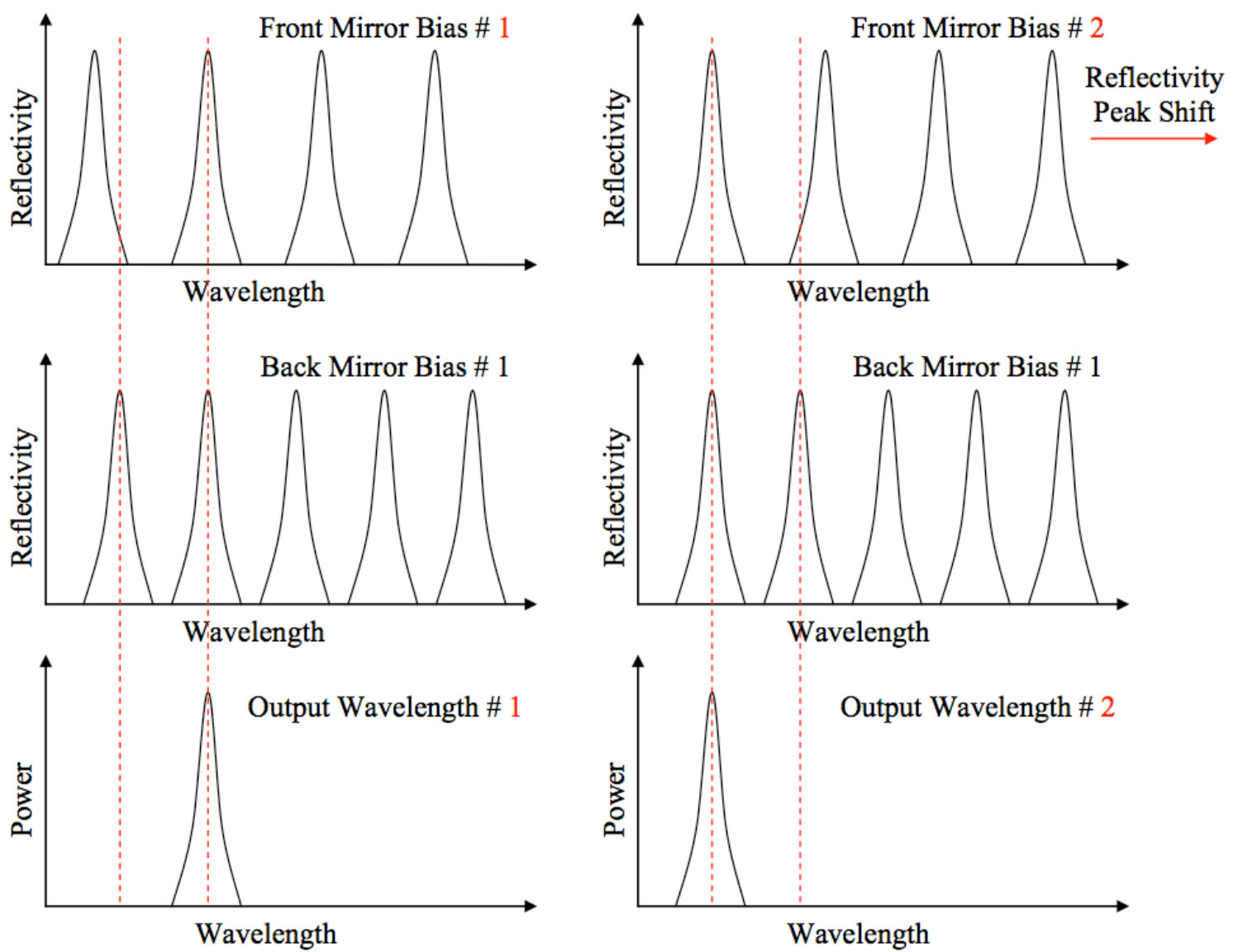

Figure 2.9: Example of the shift in wavelength due to changing the current in the front mirror section. The difference between output wavelength \#1 and output wavelength \#2 is one mode hop (5 nm) [2].

\subsection{Simultaneous Front Mirror and Back Mirror Tuning}

Mode hops of 300 picometers (pm) can be achieved when tuning the front mirror and back mirror simultaneously along the paths mentioned in Figure (2.4). Mode hops of $300 \mathrm{pm}$ are due to the cavity mode spacing of the SG-DBR laser [5]. Figure (2.10) demonstrates simultaneous front mirror and back mirror tuning with 300 pm mode hops. 


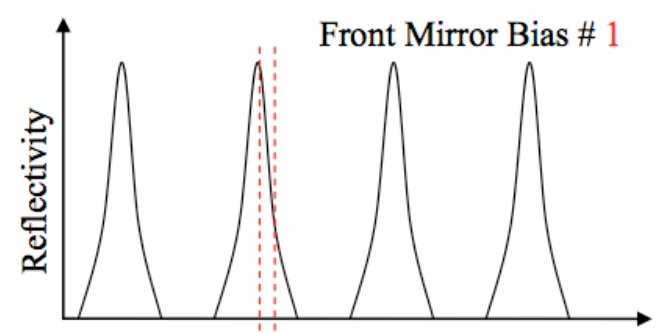

Wavelength

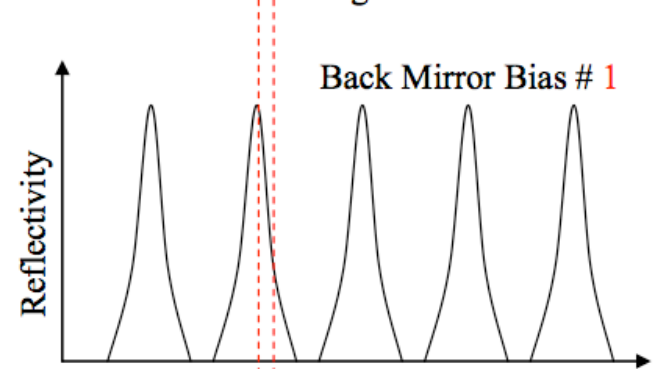

Wavelength

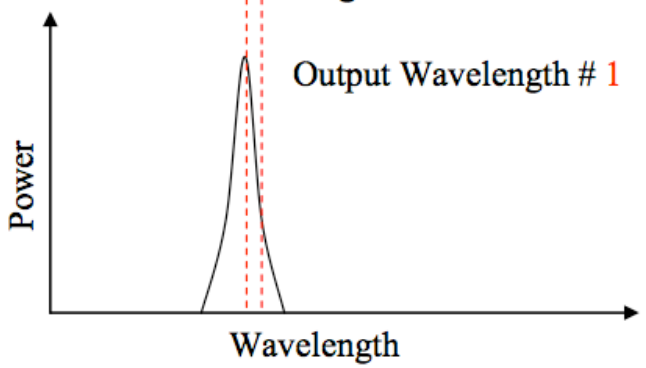

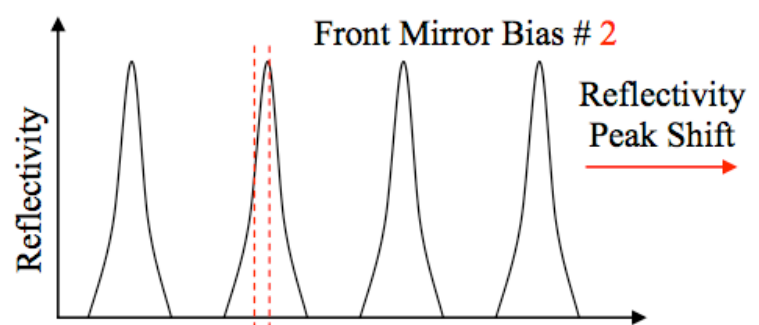

Wavelength

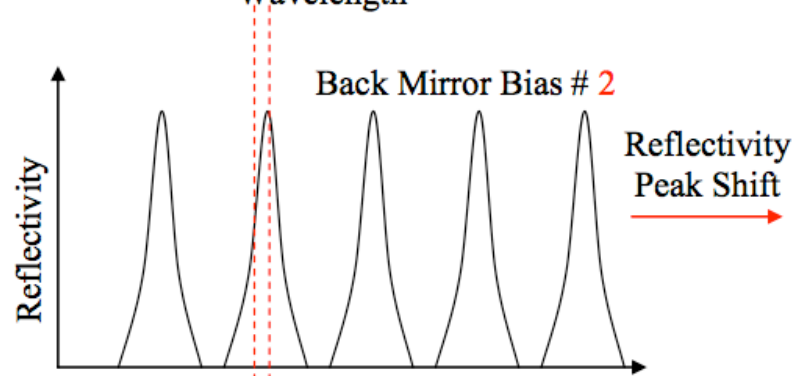

Wavelength

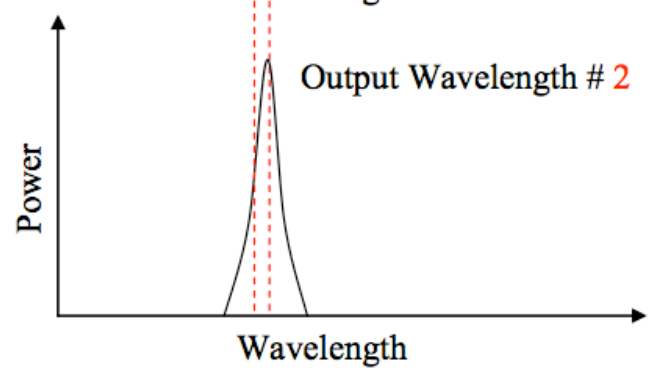

Figure 2.10: Example of the shift in wavelength due to changing the current in the front mirror and back mirror sections simultaneously. The difference between output wavelength \#1 and output wavelength \#2 is one mode hop $(0.3 \mathrm{~nm})$ [2].

\subsection{Simultaneous Front Mirror, Back Mirror, and Phase Tuning}

Since simultaneous tuning of the front mirror and back mirror can only achieve mode hops equal to the cavity mode spacing, a third tuning method is needed to attain continuous tuning. The phase section is used to create continuous tuning. The phase section tunes as the front mirror and back mirror do, by injecting current that changes the refractive index in the section. The longitudinal modes are determined by Equation 2.1 and so by changing the refractive index in the cavity, the laser output wavelength can be continuously tuned within a small bandwidth [1][4]. 
Decreasing the current into the phase section increases the laser output wavelength. Figure (2.11) demonstrates how the change of refractive index in the phase section affects the modes of the laser output. Image "a" of Figure (2.11) to image "c" shows the continuous tuning within a $300 \mathrm{pm}$ cavity mode from the low wavelength side (image "a") to the center of the mode (image "b") to the high wavelength side (image "c"). If the current is decreased past the value shown in image "c", the mode will "jump" back to the mode shown in image "a". Image "d" graphs the wavelength of the laser output, as the current into the phase section is monotonically decreased [2].
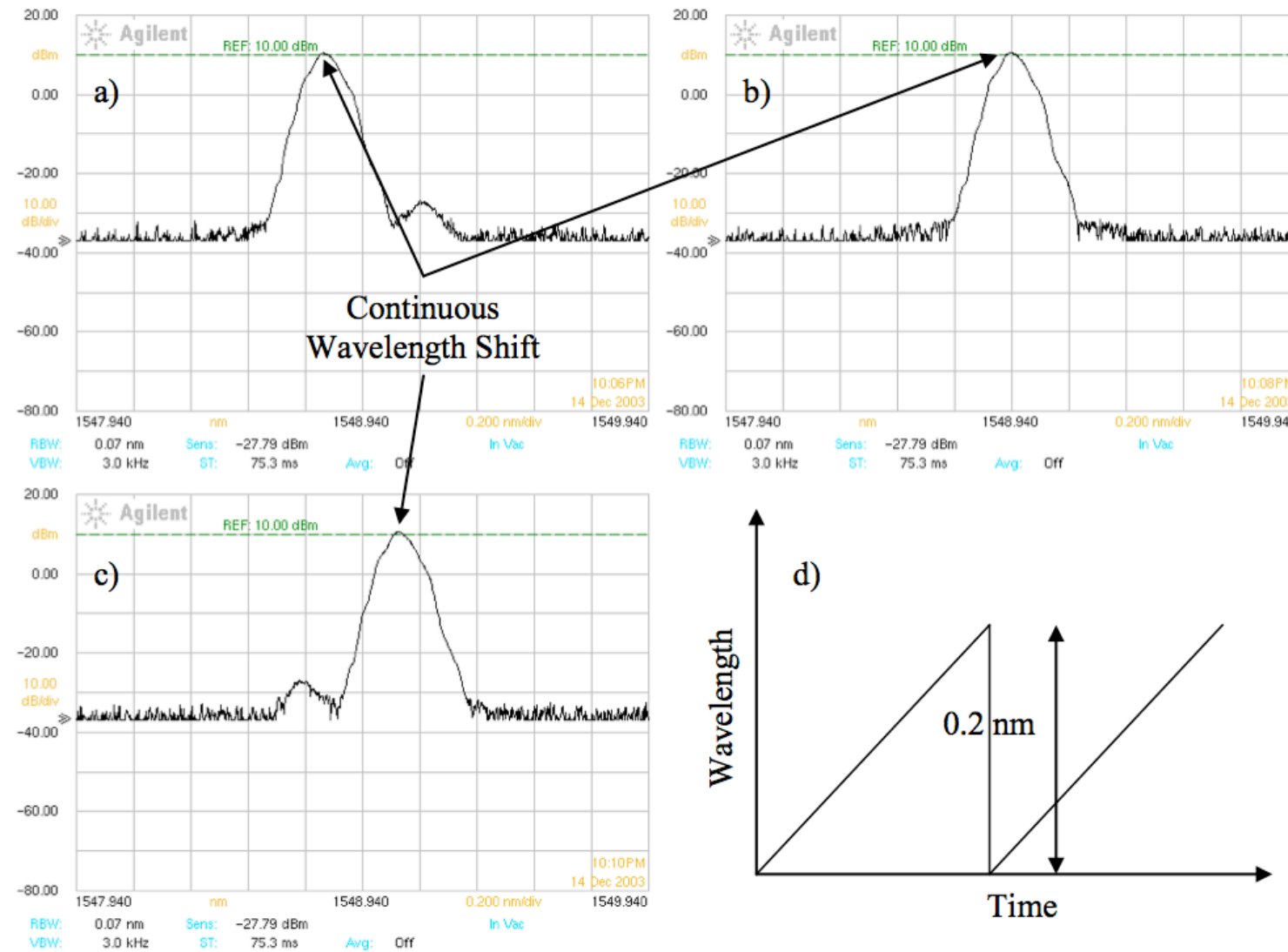

Figure 2.11: Decreasing the current into the phase section relates to an increase of wavelength depicted in images "a", "b", and "c". Image "d" shows the tuning range the phase section has before the output switches between modes [2]. 
The 300 pm mode hopping simultaneous front mirror and back mirror section tuning added with the continuous phase section tuning, gives a method for continuously tuning the laser from the minimum value of the bandwidth of the laser to the maximum value [2]. This method will be adapted and expanded upon in Chapter 4 to create a linear frequency ramp.

Some of the characteristics of the SG-DBR laser have been shown in this chapter. The five sections of the SG-DBR laser give flexibility in the wavelength tuning and output power of the laser. This flexibility will be useful when using the SG-DBR laser as a swept frequency OCT source. 


\section{CHAPTER 3 - Optical Coherence Tomography Background}

New medical optical imaging techniques are currently being adapted from older microwave remote sensing applications, such as RADAR [6]. RADAR cannot be used for medical applications, since microwave and millimeter wave radiations are highly attenuated by water or human tissue [6]. RADAR sensing techniques can be adapted for use with optical wavelengths that penetrate tissue approximately 3 millimeters $(\mathrm{mm})$. The penetration depth of the light depends on the output power of the source, wavelength of the source, and the type of material imaged. This type of optical imaging used for short range measurements is known as Optical Coherence Tomography (OCT). For medical imaging, OCT uses interferometry to extract reflectivity versus distance information of the structures being imaged [7]. Figure (3.1) shows an example of how an OCT test can image a structure in one-dimension. 


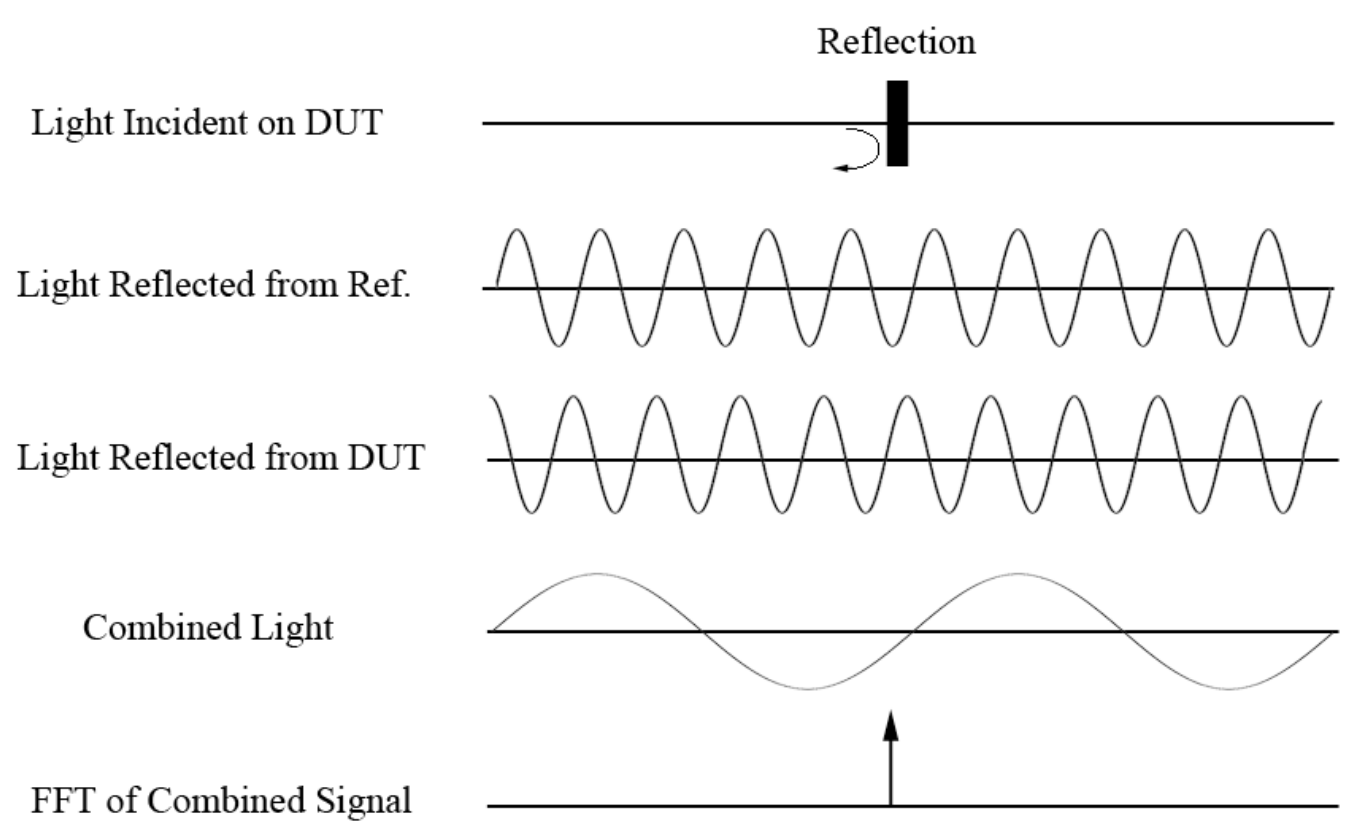

Figure 3.1: One-dimensional OCT test example showing how reflected signals give distance information about the test sample. A reflection in the DUT combined with the reference reflection gives a beat signal. Performing a FFT on the beat signal results in a delta function at the frequency of the beat signal. See Figure (3.6) for more a frequency domain representation of this figure. Figure not to scale.

One-dimensional, two-dimensional, or three-dimensional images can be created using OCT measurements. A one-dimensional OCT image consists of a single pixel imaged in the X-Y plane (parallel with imaging surface) and a set amount of pixels in the Z-direction (into the imaging material). A two-dimensional OCT test contains the set number of pixels in the Z-direction again and adds a set number of pixels in the $\mathrm{X}$ direction or Y-direction. This gives a cross sectional image of the test material, see Figure (3.2) for a sample two-dimensional OCT image. A two-dimensional OCT test consists of a set number of one-dimensional OCT tests along a line. A three-dimensional OCT image includes the two-dimensional data and then adds the missing X-direction or Y-direction previously missing from the two-dimensional image. A three-dimensional 
OCT test is a series of one-dimensional OCT tests in a plane. A common size for a threedimensional OCT image is $500 \times 500 \times 500$ pixels [8].

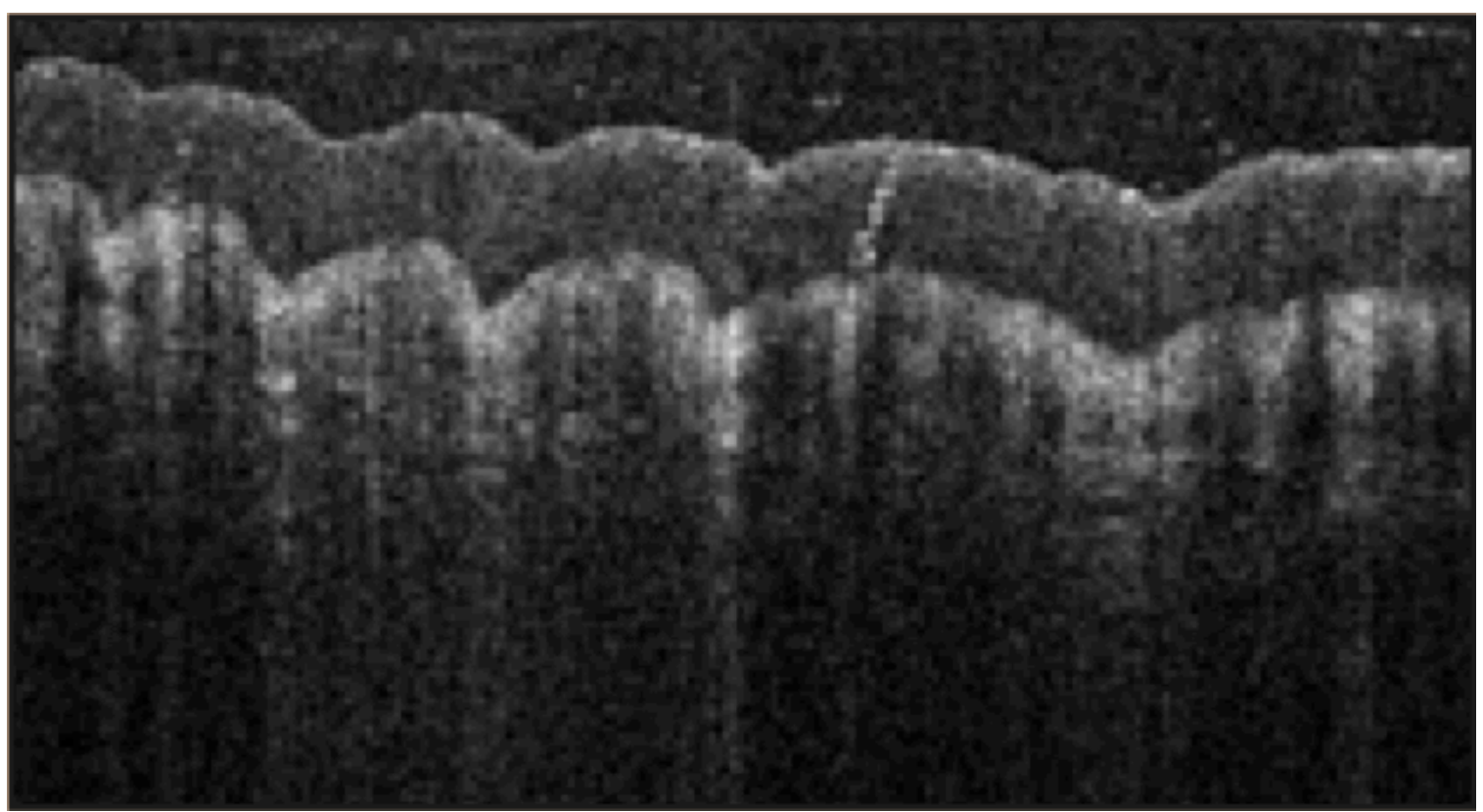

Figure 3.2: Sample two-dimensional OCT image of the skin of a human finger. Dimensions of the image are $5 \mathrm{~mm}$ (width) by $2.5 \mathrm{~mm}$ (depth) [8].

\section{1 - White-Light Interferometry}

White-Light Interferometry uses a low coherence source, such as a light emitting diode with a movable mirror and a Michelson interferometer. Figure (3.3) shows a White-Light Interferometry test setup for an OCT measurement on the device under test (DUT).

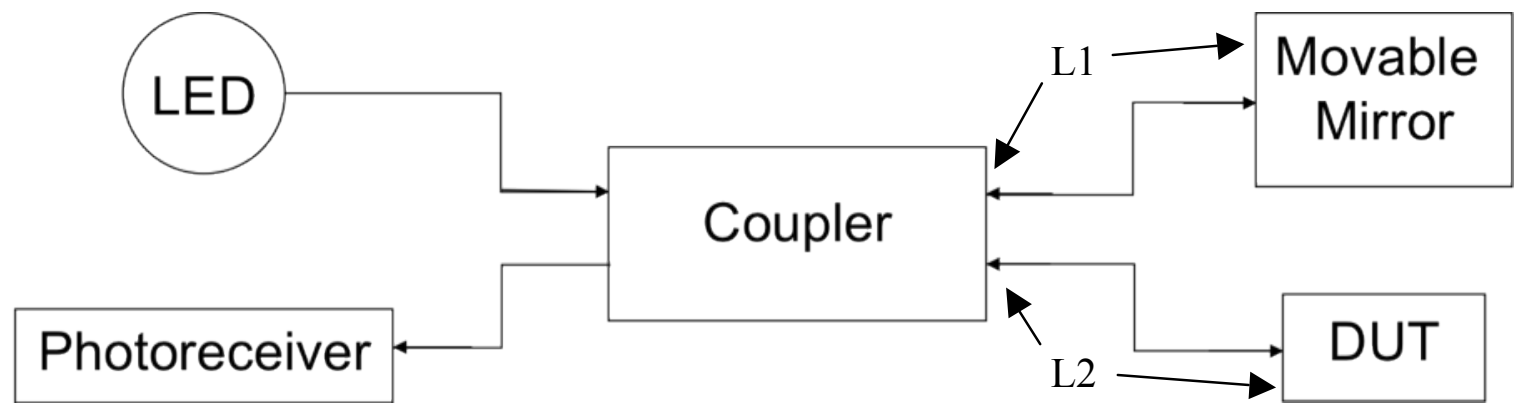

Figure 3.3: White-Light Interferometry test setup block diagram. The LED provides the low coherence source. The movable mirror scans over the small coherence range, which will change $L 1$ and change the interference between the reflected signals from the mirror and the DUT. See Figure

(3.4) to see how the interference between the two signals changes as the movable mirror shifts. 
When the distance between the coupler and the movable mirror change with respect to the distance between the coupler and a reflection from the DUT, constructive and destructive interference will occur. Also, when the mirror is moved out of the coherence range of the source, only a level power is observed [9]. Figure (3.4) shows the results of a sample White-Light Interferometry test.

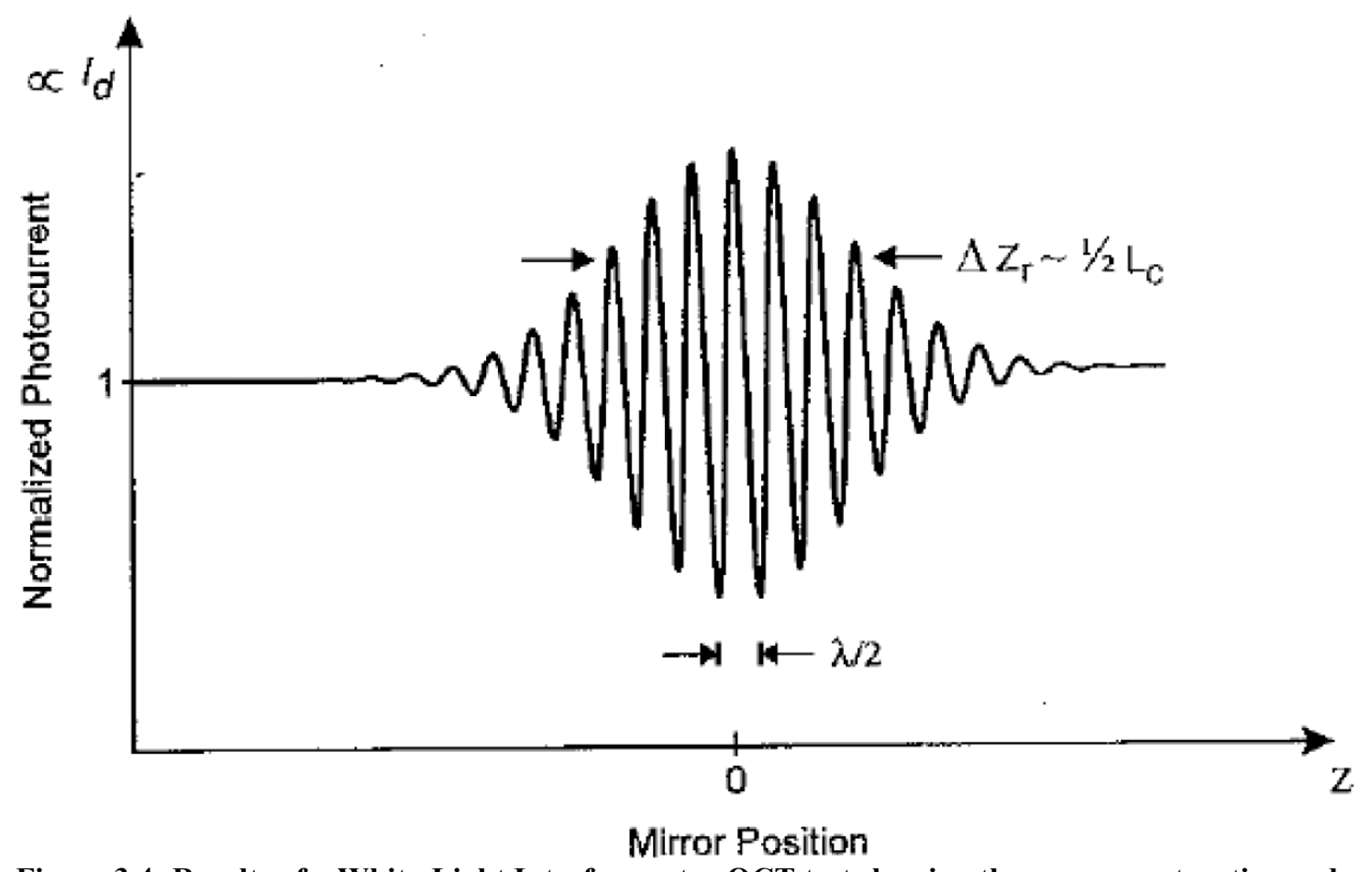

Figure 3.4: Results of a White-Light Interferometry OCT test showing the many constructive and destructive interference peaks, which repeat every $0.5 \lambda$. The maximum range of mirror position, $\Delta Z_{r}$ is given above as approximately half the coherence length of the source, $L_{c}$. See Figure (3.3) for the test setup used to create this plot [9].

White-Light Interferomtery can be useful, but it has drawbacks. Using a lowcoherence source results in a poor signal to noise ratio. This occurs since the approximately $10 \mathrm{dBm}$ output power is spread over the entire spectral range of the source. Another issue stems from the need for a movable mirror in the testing. Movable components can be expensive and are also usually slow. As stated before, a common 
OCT measurement is a $500 \times 500 \times 500$ pixel three-dimensional image. Real time OCT images are usually desired for medical applications. To calculate how fast the mirror would have to move to construct the $500 \times 500 \times 500$ pixel image at a common refresh rate, use the following.

$$
f_{\text {repetition }}=\frac{W H}{T}
$$

Where $\mathrm{W}$ is the image width in pixels, $\mathrm{H}$ the image height in pixels, and $\mathrm{T}$ the time needed to create the image. For a twenty second refresh time (as used in [8]), a width of 500 pixels, and a height of 500 pixels the mirror would have to move across its entire range 12,500 times per second. This is a difficult speed to achieve for movable components.

\section{2 - Swept Source OCT}

Most new OCT optical sensing technologies use a swept frequency source. A swept frequency optical source consists of a chirped coherent light source that ramps through a range of frequencies. Using Swept Source Interferometry, fast frequency ramps replace the action of the movable mirror. This method measures the magnitude and time delay of interference spectrums by detecting the reflected light from the sample [8]. Since common technologies currently exist to sweep the range of frequencies of the source at rates of $16 \mathrm{kHz}$ and above, Swept Source Interferometry has become the primary method for OCT measurements. Swept source OCT also has much high signal to noise ratios since the output power of the laser is mostly contained within the narrow linewidth of the source. Figure (3.5) shows the test setup used for a Swept Source OCT measurement. 


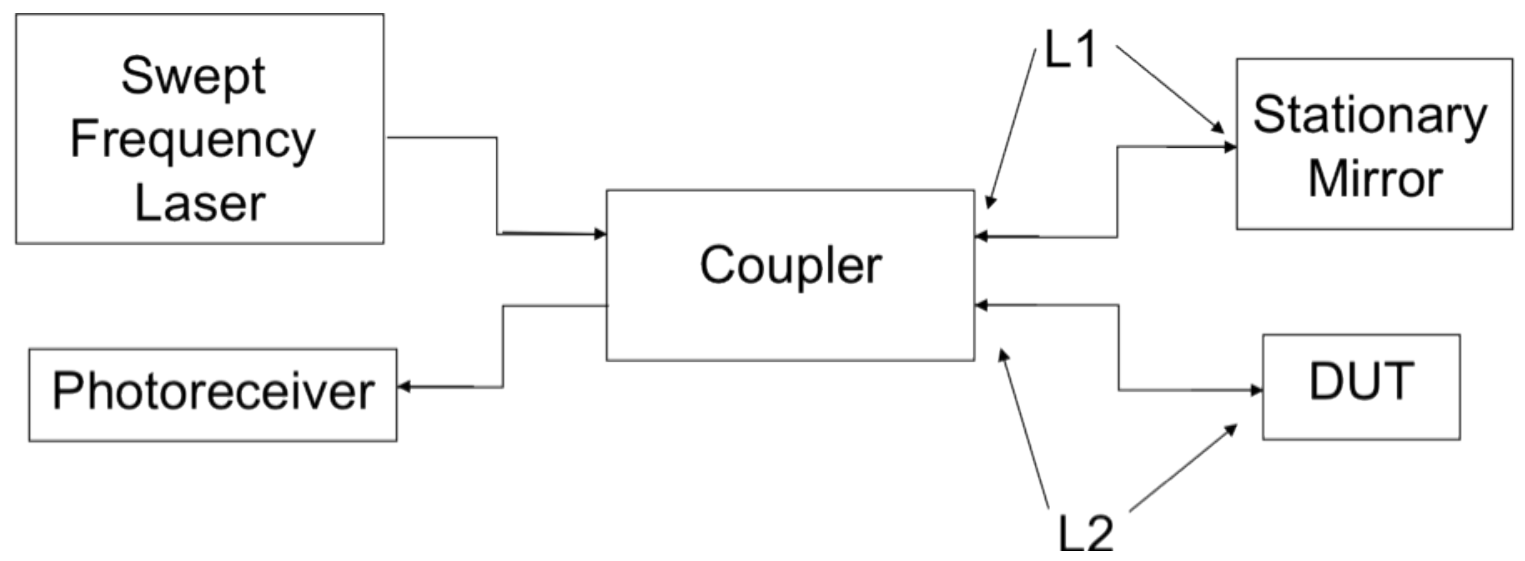

Figure 3.5: Swept Source OCT test setup. Movable mirror, shown in Figure (3.3), is replaced with a stationary mirror and the low coherence source is replaced with a high coherence swept frequency laser.

Figure (3.6) shows the two signals present at the photoreceiver if the DUT is a mirror. The two signals in Figure (3.6) interfere and the result is a sinusoid with frequency, $\mathrm{f}_{\text {photoreceiver }}$ that is equal to $\Delta \mathrm{f}$. 


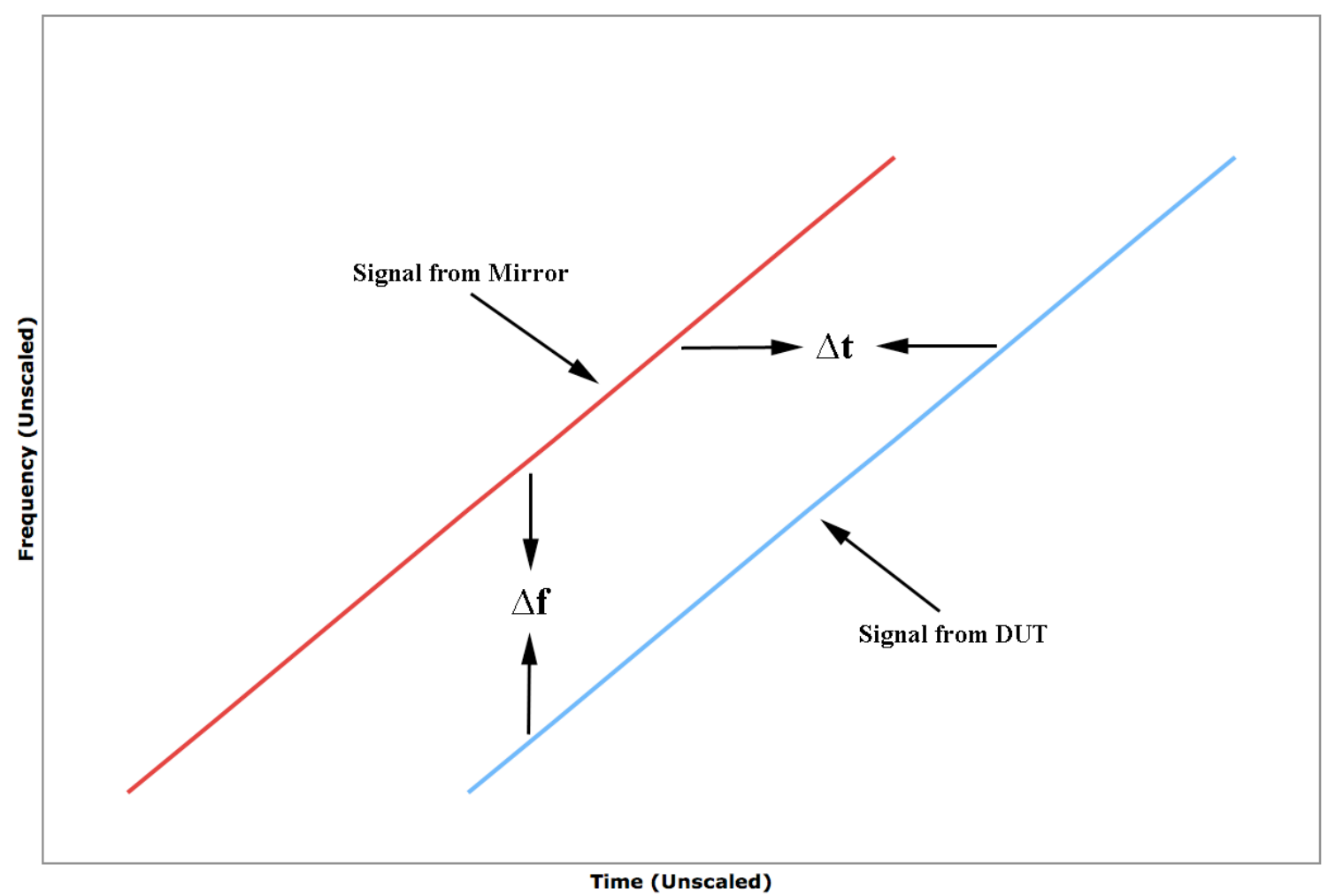

Figure 3.6: Combined signals from the stationary mirror and the DUT. $\Delta t$ is the time difference between the path lengths $L 1$ and $L 2$. $\Delta f$ is the beat frequency between the two signals, which also gives the time delay of the DUT signal. See Figure (3.1).

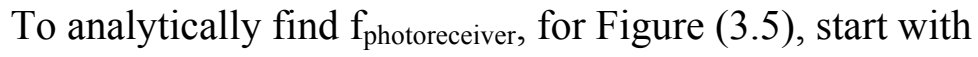

$$
t=\frac{d}{v}
$$

where $\mathrm{t}$ is time, $\mathrm{d}$ is distance, and $\mathrm{v}$ is the speed of light in the medium. Since the signal reflects off the mirror and DUT, $d=L_{1}$ and $d=L_{2}$ respectively. Subtracting Equation (3.2) for the stationary mirror from Equation (3.2) for the DUT to get Equation (3.3).

$$
t_{1}-t_{2}=\frac{2 L_{1}}{v}-\frac{2 L_{2}}{v}
$$

Simplifying Equation (3.3) obtains

$$
\Delta t=\frac{2 \Delta L}{v}
$$

where $\Delta \mathrm{t}=\mathrm{t}_{1}-\mathrm{t}_{2}$ and $\Delta \mathrm{L}=\mathrm{L}_{1}-\mathrm{L}_{2}$. The slope of the lines in Figure (3.5) equal 


$$
m=\frac{\Delta f}{\Delta t}
$$

Putting these equations together gives the frequency of the combined signal at the photodiode.

$$
f_{\text {photodiode }}=\left[\frac{2 \Delta L}{v}\right]\left[\frac{\Delta f}{\Delta t}\right]
$$

Inserting Equation (3.4) into Equation (3.6) gives Equation (3.7).

$$
f_{\text {photodiode }}=\Delta f
$$

Using Swept Source Interferometery, the time resolution of the measurement depends on the source. The primary parameters for a proper swept source laser are repetition rate, coherence length (which depends on the laser linewidth), output power, laser spectral coverage, and the linearity of the laser frequency ramp.

The laser linewidth, which influences the coherence length of the laser, is a property of the source chosen and cannot be changed easily. Output power is also dependant on the type of laser chosen, however, optical amplifiers can increase the output power if necessary. The laser spectral coverage depends on several factors but usually can be increased to larger values [2][8]. The linearity of the laser frequency ramp depends entirely on how the swept frequency source is implemented. Any non-linearity of the swept frequency source will decrease the resolution of the OCT measurement as will be shown in Chapter 7.

The spectral coverage of the laser relates to the bandwidth of the frequency sweep. This parameter, in particular, is important because the distance resolution of an OCT measurement is directly controlled by the bandwidth. Equation (3.8) exhibits this relationship [10]. 


$$
\Delta R=\frac{c}{2 B}
$$

where $\Delta \mathrm{R}$ is the distance resolution of the OCT measurement, $\mathrm{c}$ is the speed of light in vacuum, and $\mathrm{B}$ is the laser tuning range. When choosing a swept frequency OCT source, a trade off exists between the spectral coverage and the repetition rate of the laser, at some maximum tuning rate of the laser in gigahertz per second. As the bandwidth for any specific source doubles, the repetition rate will be halved. A specific repetition rate and bandwidth should be optimized depending on the specific application. 


\section{CHAPTER 4 - Frequency Calibration of a L-band and C-band Laser}

As stated in Chapter 3, OCT measurements require a highly linear frequency ramp with fast repetition rates. Chapter 4 details methods to create a linear frequency ramp for a L-band and a C-band SG-DBR laser. A few separate characterizations and analyses are required to generate the needed frequency ramp.

In Section 4.1, a two-dimensional laser mode map must be created for each laser. This map is a three-dimensional plot with the $\mathrm{X}-\mathrm{Y}$ plane representing the range of currents into the front mirror and back mirror sections and mapping the wavelength for a set number of points in the Z-axis. Tuning "paths" are chosen in the two-dimensional space to cover the entire bandwidth of the laser.

Next in Section 4.2, approximately 500 set points are selected along these tuning paths and the phase section is manually changed, at each point, to give optimum suppression of side modes along these points. This process is called three-dimensional characterization.

The last step to create a linear frequency ramp is to interpolate these points in linear frequency steps in Section 4.3. This process will give a "tuning dictionary" in which to ramp the laser linearly in frequency.

In Section 4.4, the new waveforms are inputted to the SG-DBR laser to create a linear frequency ramp for the L-band and C-band lasers.

\subsection{Two-Dimensional Characterization}

The LabVIEW program used to characterize the laser modes was originally created by Shane O'Conner and was passed down for others to use [2]. Two-dimensional characterization involves describing the laser output wavelength versus front mirror and 
back mirror currents. The block diagram used for the two-dimensional characterization of the laser modes is shown in Figure (4.1).

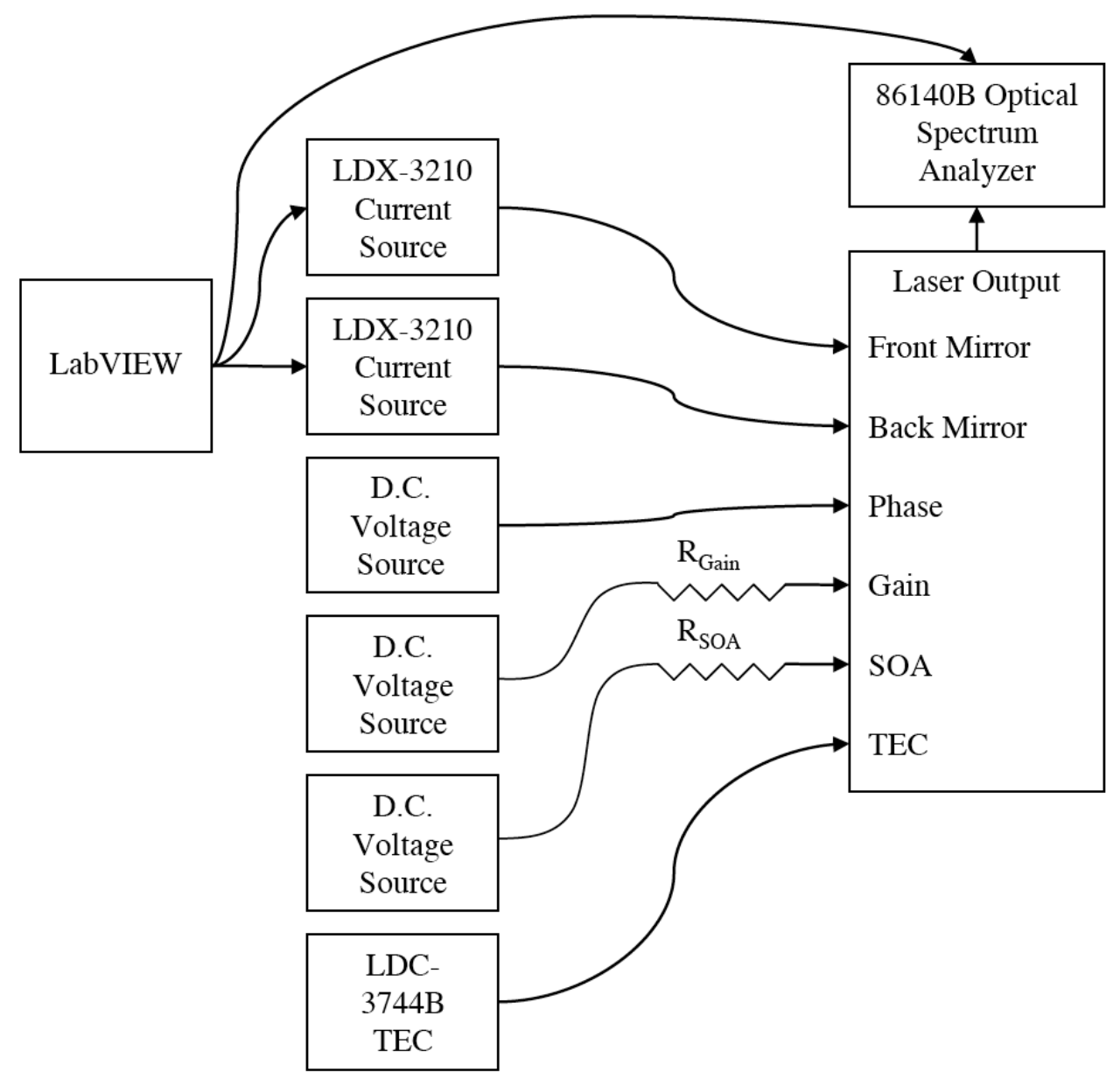

Figure 4.1: Block Diagram of the two-dimensional laser characterization setup configuration. The gain and SOA sections are kept at constant bias current while the phase voltage is set to zero. The currents into the front mirror and back mirror are set by constant current sources and are controlled by LabVIEW.

The LabVIEW program is designed to ask for the range of front mirror and back mirror currents as well as the step size between increments in front mirror and back mirror currents. The other three inputs are controlled by D.C. voltage sources. The gain section is biased at 100 milliamps $(\mathrm{mA})$, the SOA section is biased at $150 \mathrm{~mA}$, and the phase section input voltage is set to 0 volts $(\mathrm{V})$ for this test. Due to the large maximum input currents of the front mirror and back mirror sections $(35 \mathrm{~mA}$ and $60 \mathrm{~mA}$ 
respectively) and the need for high resolution (small step size increments), two separate tests were performed. The first test was a "fine" resolution test, characterizing each mirror from $0 \mathrm{~mA}$ to $12.5 \mathrm{~mA}$ at increments of $0.125 \mathrm{~mA}$. The second test was a "normal" resolution test, characterizing each mirror from $0 \mathrm{~mA}$ to the maximum current in the section at step sizes of $0.5 \mathrm{~mA}$. At approximately 7 seconds per measurement, the fine and normal resolution test durations are 19.83 and 17.70 hours respectively.

The resulting output file from the LabVIEW program gives three-dimensional data that when plotted gives a staircase type function with laser wavelength plotted on the Z-axis. The plot in Figure (4.2) (L-band) and Figure (4.3) (C-band) show that varying the front mirror and back mirror currents can cover all the laser bandwidth for the normal resolution characterization.

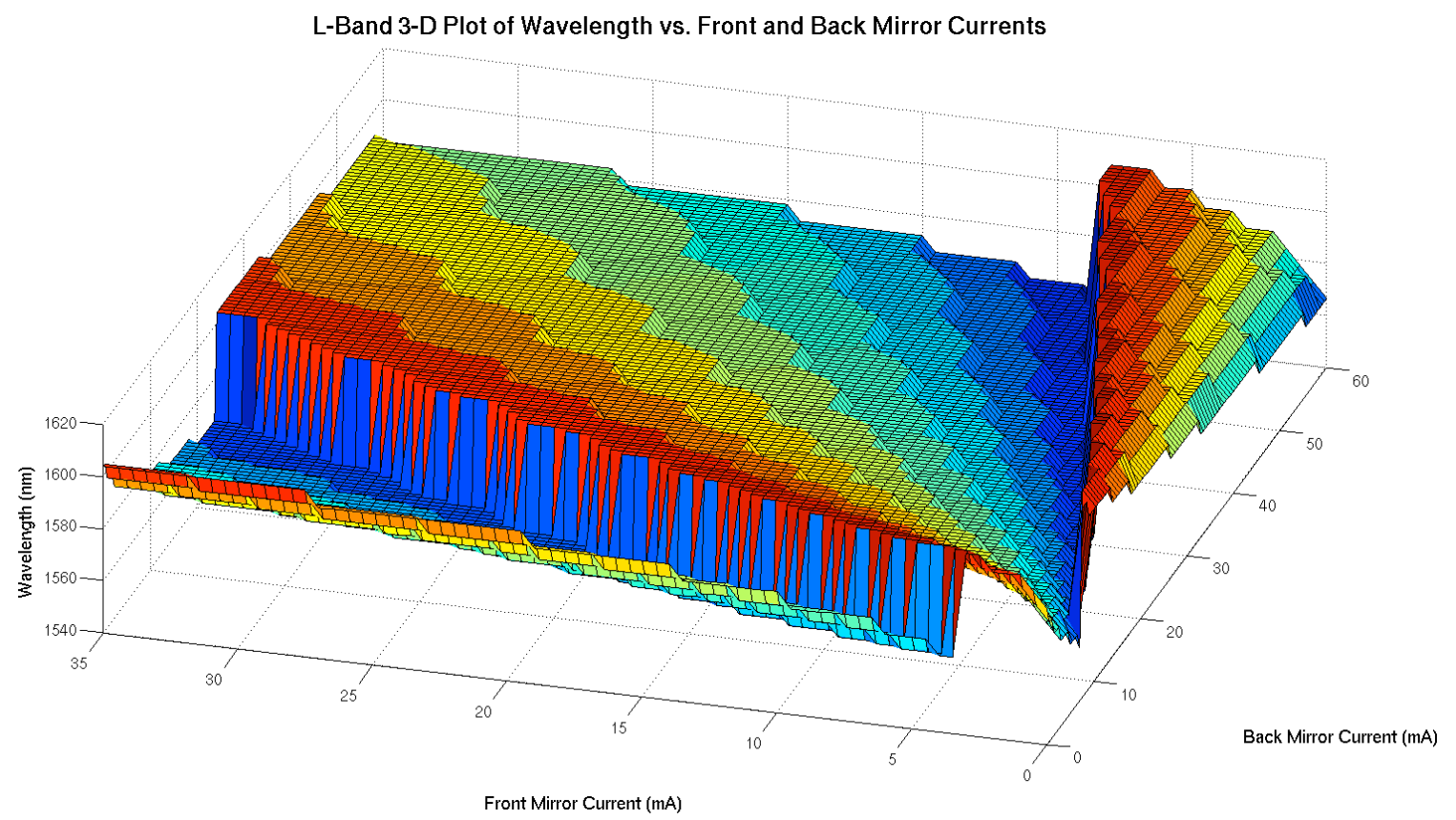

Figure 4.2: Three-dimensional (mode map) plot of wavelength vs. mirror currents for the L-band laser. Each region of color represents a tuning path to create a continuous wavelength ramp. See Figure (4.5) to see the eight tuning paths chosen to cover the wavelength tuning range of the laser. 


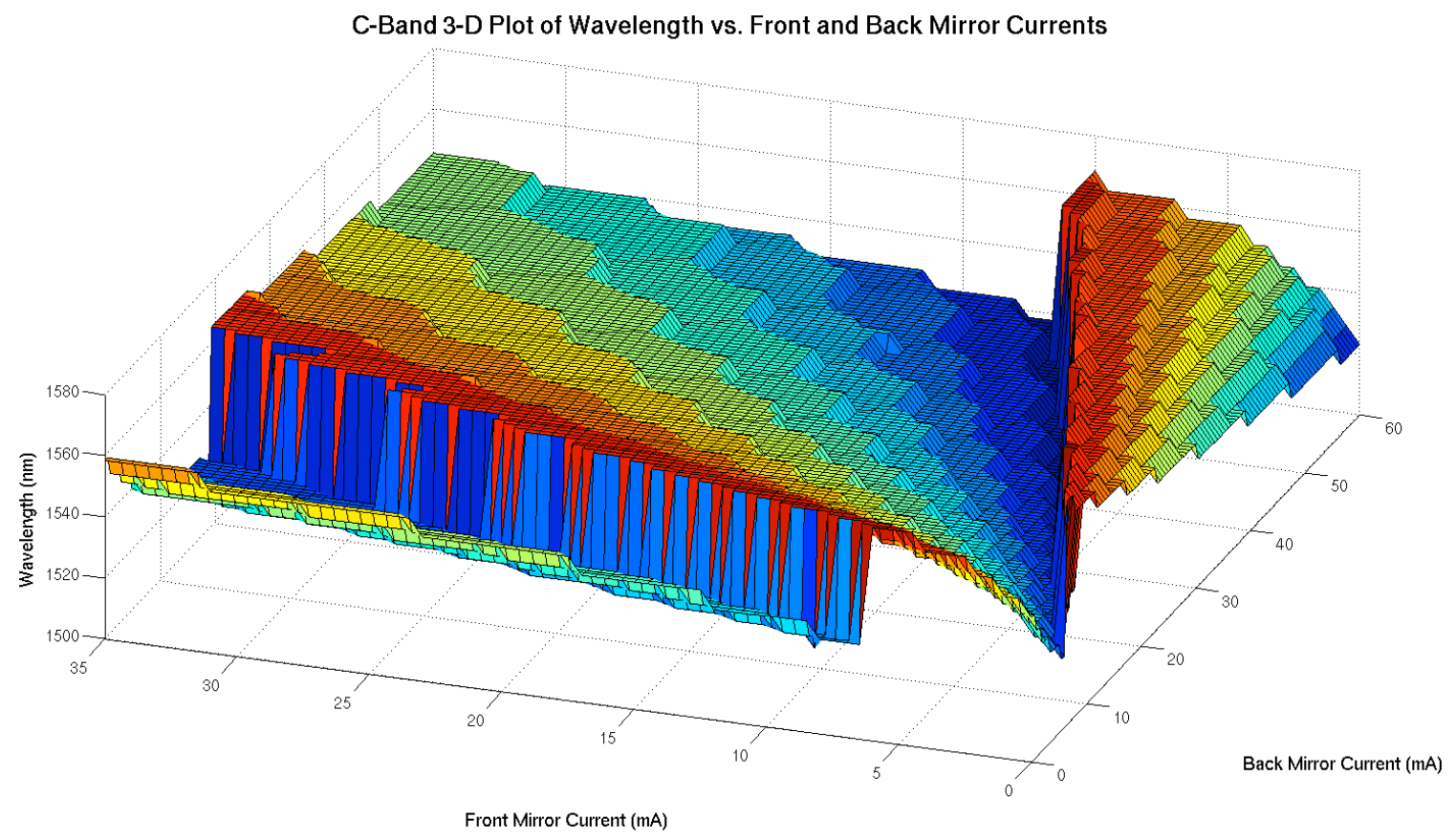

Figure 4.3: Three-dimensional (mode map) plot of wavelength vs. mirror currents for the C-band laser. Each region of color represents a tuning path to create a continuous wavelength ramp. See Figure (4.7) to see the eight tuning paths chosen to cover the wavelength tuning range of the laser.

The areas of like color in Figure (4.2) and Figure (4.3), are called tuning paths since following a tuning path will give near continuous wavelength tuning with $300 \mathrm{pm}$ jumps. The range of paths along the steps from low to high in the center of each plot (see Figure (4.5) and Figure (4.7) to see paths one through eight) are what is used to tune the laser from low wavelengths to high wavelengths over the bandwidth of the laser [2]. This data is re-plotted using a contour plot in Figure (4.4), Figure (4.5), Figure (4.6), and Figure (4.7). Figure (4.4) and Figure (4.5) are the contour plots for the L-band "fine" resolution and "normal" resolution respectively. Figure (4.6) and Figure (4.7) are the contour plots for the C-band "fine" resolution and "normal" resolution respectively. Figure (4.4) and Figure (4.6) characterize the area below 12.5 milliamps input current, with a smaller step size, for Figure (4.5) and Figure (4.7) respectively. 


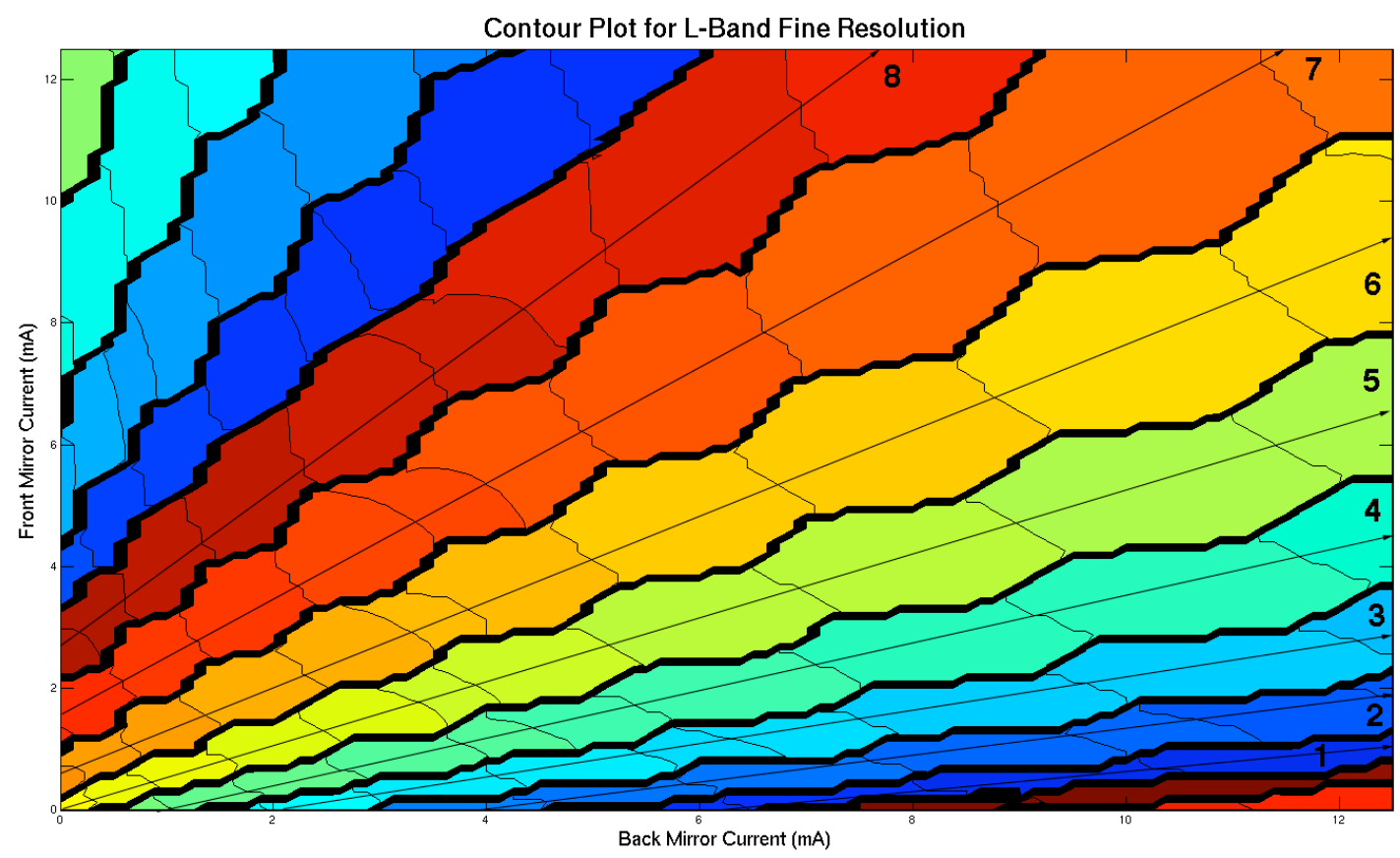

Figure 4.4: Contour plot for $\mathrm{L}-$ band laser showing fine resolution characterization up to $12.5 \mathrm{~mA}$ input current in the mirrors. Note the eight tuning paths covering low wavelengths to high wavelengths.

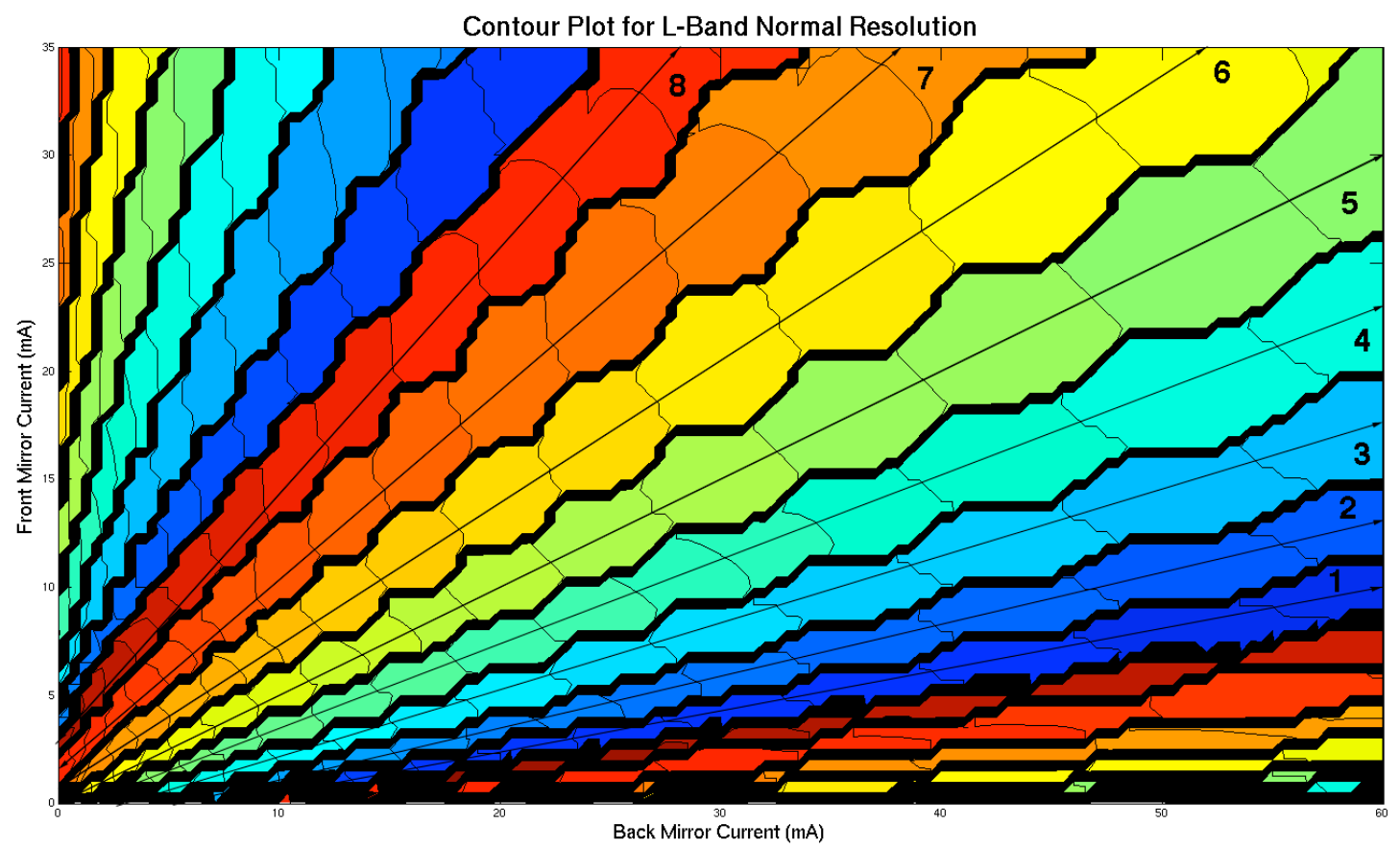

Figure 4.5: Contour plot for L-band laser showing normal resolution characterization up to the maximum current in the mirrors. Note the eight tuning paths covering low wavelengths to high wavelengths. Also see Figure (4.2) to see a three-dimensional plot of this data. 


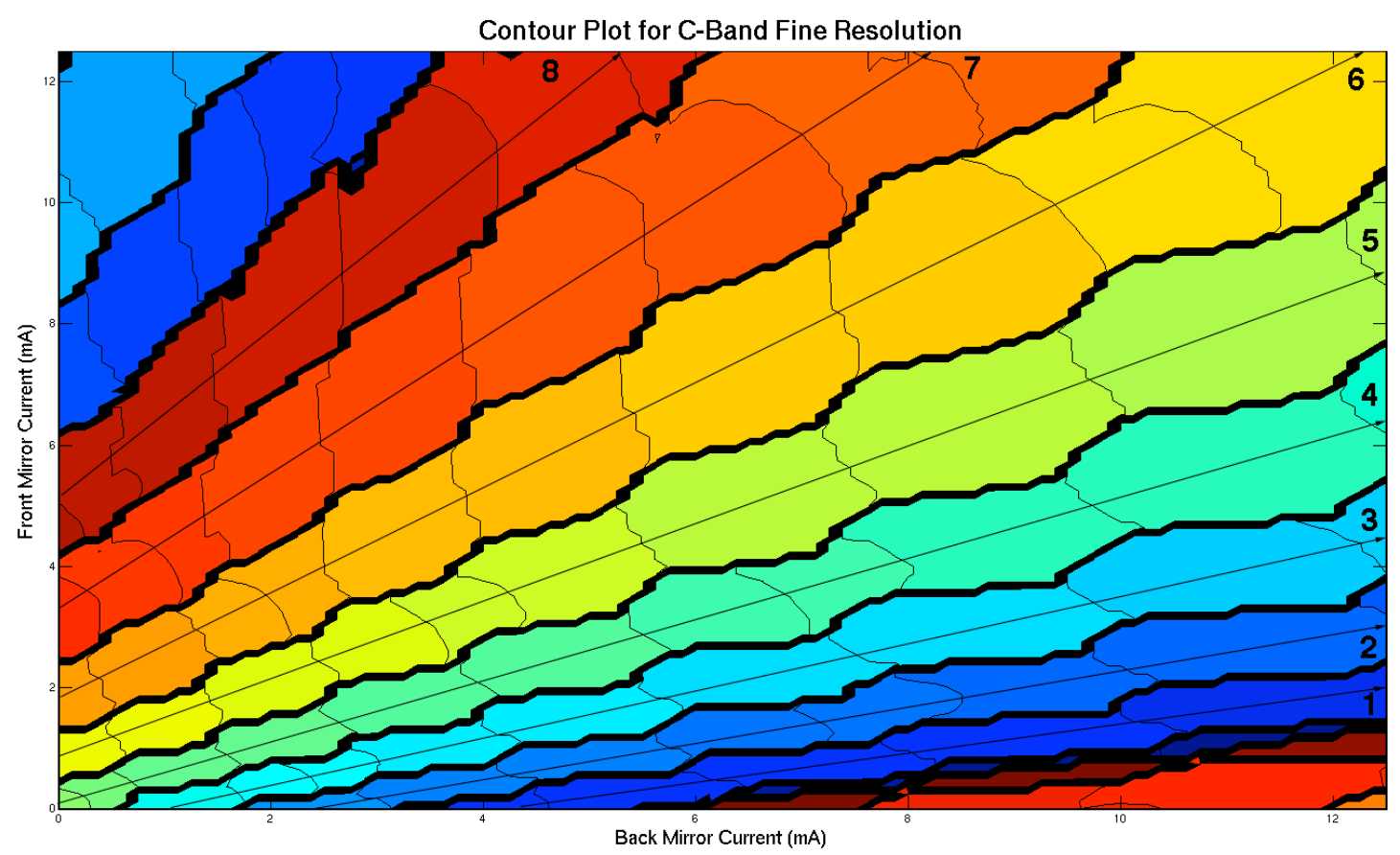

Figure 4.6: Contour plot for C-band laser showing fine resolution characterization up to $12.5 \mathrm{~mA}$ input current in the mirrors. Note the eight tuning paths covering low wavelengths to high wavelengths.

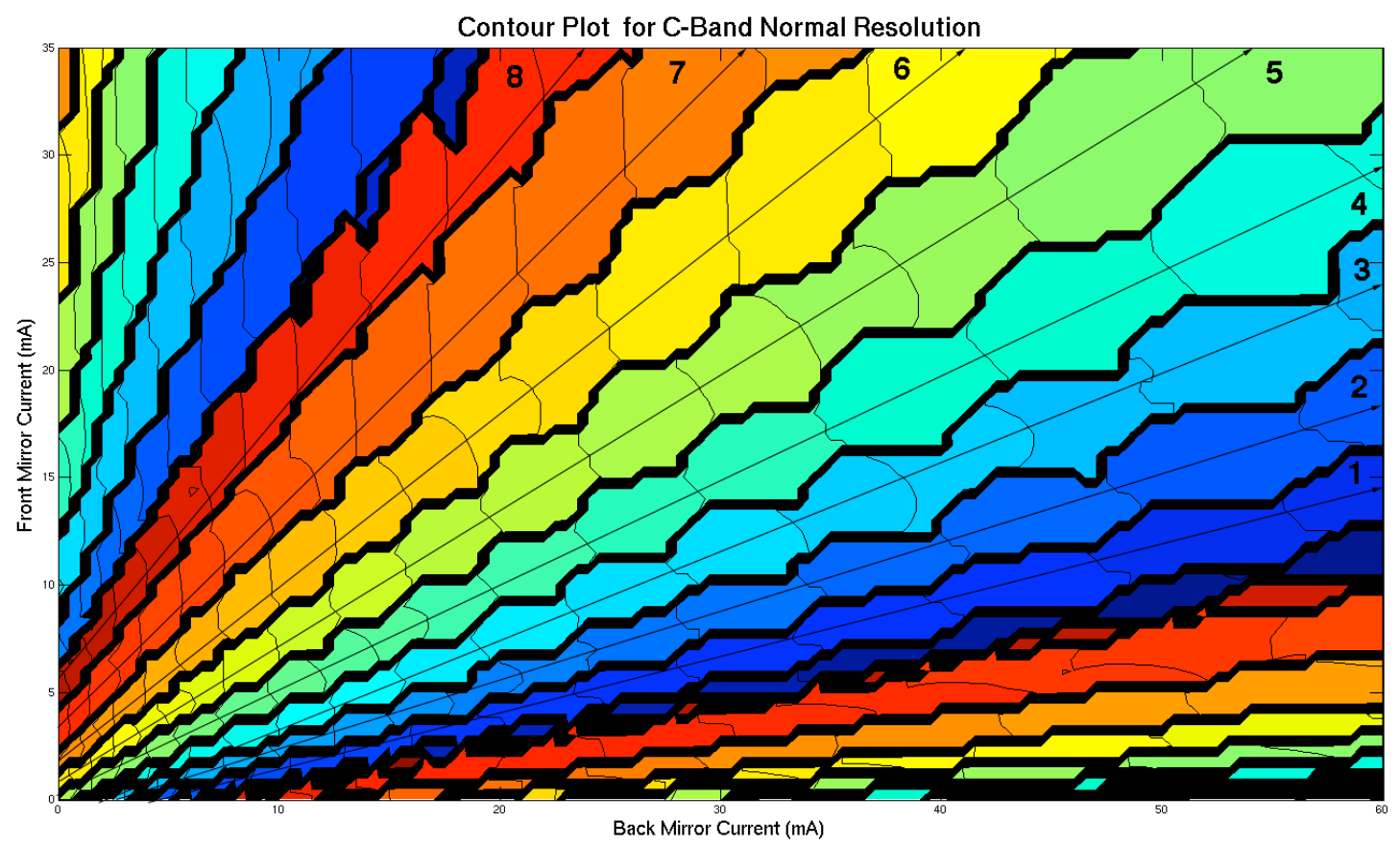

Figure 4.7: Contour plot for C-band laser showing normal resolution characterization up to the maximum current in the mirrors. Note the eight tuning paths covering low wavelengths to high wavelengths. Also see Figure (4.3) to see a three-dimensional plot of this data. 
Figure (4.4) and Figure (4.6) are used since the width of each tuning path decreases as both the front mirror and back mirror currents decrease. Care must be taken when describing tuning paths to ensure that the laser output does not change modes.

Another less obvious feature of these mode maps consists of a quadratic wavelength versus tuning path attribute, which is expanded upon in Figure (4.8).

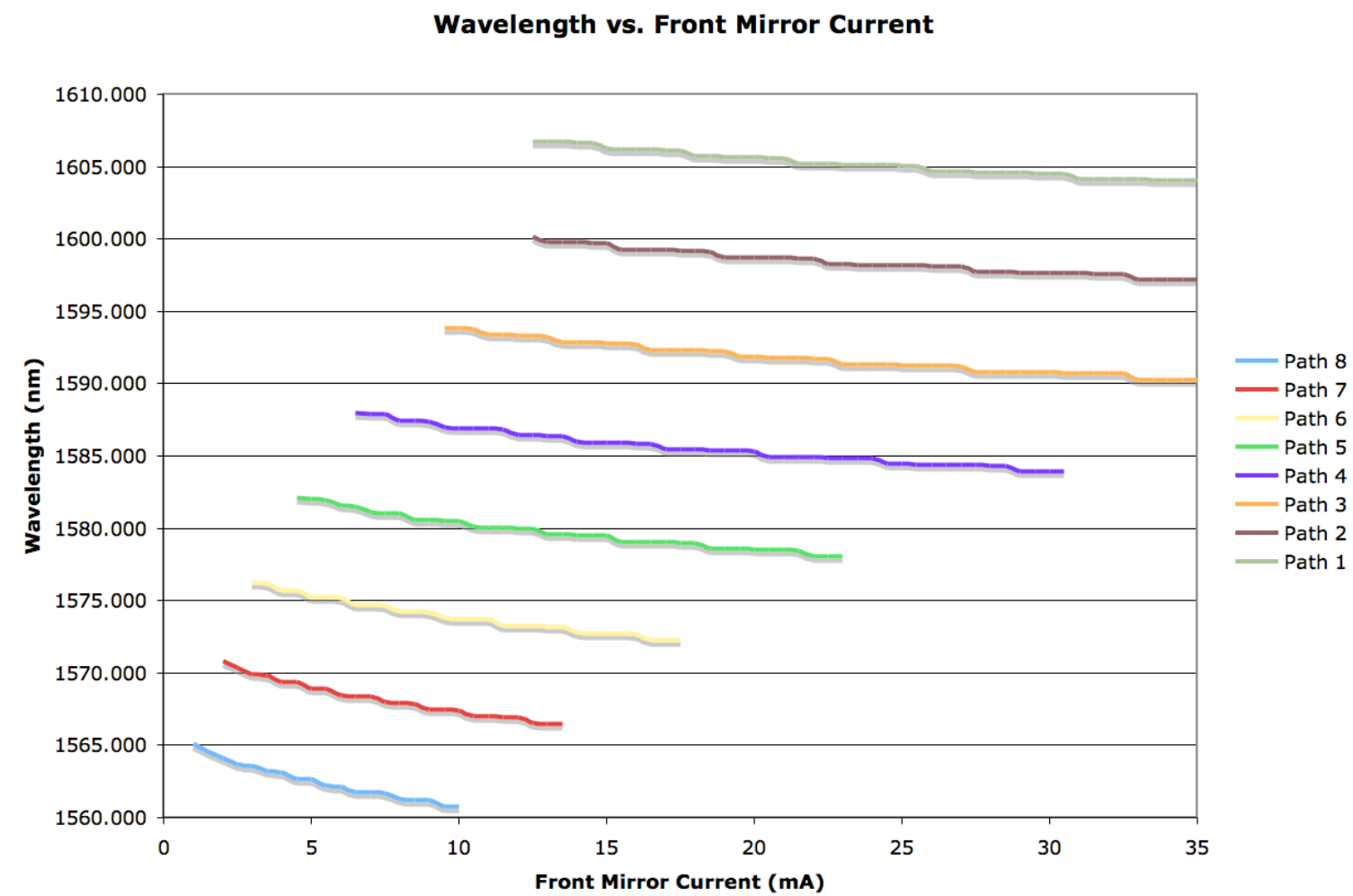

Figure 4.8: L-band laser plot showing the wavelength versus current curves. The have a slightly quadratic nature that will be approximated as linear. Also note the $0.3 \mathrm{~nm}$ mode hops along each path. Paths are referenced from Figure (4.5).

With the laser mode maps and the ideal paths drawn out, the start points and end points of each tuning path can be recorded. To take advantage of the fine characterization, Table (4.1) shows the start and end points as well as the wavelength data for the fine L-band characterization. Table (4.2) records the data from where the fine paths leave off and continues to the maximum currents available for the L-band. Table (4.3) and Table (4.4) show the same data but for the C-band. 


\begin{tabular}{|c|c|c|c|c|c|c|}
\hline \multirow{2}{*}{$\begin{array}{c}\text { Path } \\
\text { Number }\end{array}$} & \multicolumn{3}{|c|}{ Beginning of Path } & \multicolumn{3}{c|}{ End of Path } \\
\cline { 2 - 7 } & $\begin{array}{c}\text { Wavelength } \\
(\mathrm{nm})\end{array}$ & $\begin{array}{c}\mathbf{I}_{\text {back mirror }} \\
(\mathrm{m} \mathrm{A})\end{array}$ & $\begin{array}{c}\mathbf{I}_{\text {front mirror }} \\
(\mathrm{mA})\end{array}$ & $\begin{array}{c}\text { Wavelength } \\
(\mathrm{nm})\end{array}$ & $\begin{array}{c}\mathbf{I}_{\text {back mirror }} \\
(\mathrm{mA})\end{array}$ & $\begin{array}{c}\mathbf{I}_{\text {front mirror }} \\
(\mathrm{m} \mathrm{A})\end{array}$ \\
\hline $1 \mathrm{~A}$ & 1566.520 & 6 & 0 & 1565.060 & 12.5 & 1 \\
\hline $2 \mathrm{~A}$ & 1572.825 & 3.75 & 0 & 1570.800 & 12.5 & 1.75 \\
\hline $3 \mathrm{~A}$ & 1579.175 & 2.125 & 0 & 1576.225 & 12.5 & 2.875 \\
\hline $4 \mathrm{~A}$ & 1585.955 & 0.875 & 0 & 1582.065 & 12.5 & 4.375 \\
\hline $5 \mathrm{~A}$ & 1592.440 & 0 & 0 & 1587.930 & 12.5 & 6.5 \\
\hline $6 \mathrm{~A}$ & 1598.375 & 0 & 0.5 & 1593.840 & 12.5 & 9.375 \\
\hline $7 \mathrm{~A}$ & 1603.930 & 0 & 1.625 & 1599.795 & 11.375 & 12.5 \\
\hline $8 \mathrm{~A}$ & 1609.920 & 0 & 2.75 & 1606.740 & 7.625 & 12.5 \\
\hline
\end{tabular}

Table 4.1: Characterizes L-Band at the start and end points of the paths along the fine twodimensional characterization.

\begin{tabular}{|c|c|c|c|c|c|c|}
\hline \multirow{2}{*}{$\begin{array}{c}\text { Path } \\
\text { Number }\end{array}$} & \multicolumn{3}{|c|}{ Beginning of Path } & \multicolumn{3}{c|}{ End of Path } \\
\cline { 2 - 7 } & $\begin{array}{c}\text { Wavelength } \\
(\mathrm{nm})\end{array}$ & $\begin{array}{c}\mathbf{I}_{\text {back mirror }} \\
(\mathrm{mA})\end{array}$ & $\begin{array}{c}\mathbf{I}_{\text {front mirror }} \\
(\mathrm{mA})\end{array}$ & $\begin{array}{c}\text { Wavelength } \\
(\mathrm{nm})\end{array}$ & $\begin{array}{c}\mathbf{I}_{\text {back mirror }} \\
(\mathrm{mA})\end{array}$ & $\begin{array}{c}\mathbf{I}_{\text {front mirror }} \\
(\mathrm{m} \mathrm{A})\end{array}$ \\
\hline 1B & 1565.060 & 12.5 & 1 & 1560.735 & 60 & 9.5 \\
\hline 2B & 1570.800 & 12.5 & 1.75 & 1566.460 & 60 & 12.5 \\
\hline 3B & 1576.225 & 12.5 & 2.875 & 1572.235 & 60 & 16 \\
\hline 4B & 1582.065 & 12.5 & 4.375 & 1578.035 & 60 & 22 \\
\hline 5B & 1587.930 & 12.5 & 6.5 & 1583.880 & 60 & 29 \\
\hline 6B & 1593.840 & 12.5 & 9.375 & 1590.215 & 53.5 & 35 \\
\hline 7B & 1599.795 & 11.375 & 12.5 & 1597.080 & 40 & 35 \\
\hline 8B* & 1606.740 & 7.625 & 12.5 & 1604.080 & 29.76 & 35.8 \\
\hline
\end{tabular}

Table 4.2: Characterizes L-Band at the start and end points of the paths along the normal twodimensional characterization. Path 8 is extended slightly to give full wavelength coverage.

\begin{tabular}{|c|c|c|c|c|c|c|}
\hline \multirow{2}{*}{$\begin{array}{c}\text { Path } \\
\text { Number }\end{array}$} & \multicolumn{3}{|c|}{ Beginning of Path } & \multicolumn{3}{c|}{ End of Path } \\
\cline { 2 - 7 } & $\begin{array}{c}\text { Wavelength } \\
(\mathrm{nm})\end{array}$ & $\begin{array}{c}\mathbf{I}_{\text {back mirror }}(\mathrm{mA}) \\
\text { 1A }\end{array}$ & $\begin{array}{c}\mathbf{I}_{\text {front mirror }} \\
(\mathrm{mA})\end{array}$ & $\begin{array}{c}\text { Wavelength } \\
(\mathrm{nm})\end{array}$ & $\begin{array}{c}\mathbf{I}_{\text {back mirror }} \\
(\mathrm{mA})\end{array}$ & $\begin{array}{c}\mathbf{I}_{\text {front mirror }} \\
(\mathrm{mA})\end{array}$ \\
\hline $2 \mathrm{~A}$ & 1529.130 & 4.25 & 0 & 1527.235 & 12.5 & 2 \\
\hline $3 \mathrm{~A}$ & 1541.265 & 1 & 0 & 1537.915 & 12.5 & 4.625 \\
\hline $4 \mathrm{~A}$ & 1547.330 & 0 & 0.125 & 1543.470 & 12.5 & 6.5 \\
\hline $5 \mathrm{~A}$ & 1552.950 & 0 & 0.75 & 1549.065 & 12.5 & 8.75 \\
\hline $6 \mathrm{~A}$ & 1558.585 & 0 & 1.75 & 1555.010 & 12.375 & 12.5 \\
\hline $7 \mathrm{~A}$ & 1564.240 & 0 & 3.25 & 1561.295 & 8.125 & 12.5 \\
\hline $8 \mathrm{~A}$ & 1569.970 & 0 & 4.625 & 1567.830 & 5.75 & 12.5 \\
\hline
\end{tabular}

Table 4.3: Characterizes C-Band at the start and end points of the paths along the fine twodimensional characterization. 


\begin{tabular}{|c|c|c|c|c|c|c|}
\hline \multirow{2}{*}{$\begin{array}{c}\text { Path } \\
\text { Number }\end{array}$} & \multicolumn{3}{|c|}{ Beginning of Path } & \multicolumn{3}{c|}{ End of Path } \\
\cline { 2 - 7 } & $\begin{array}{c}\text { Wavelength } \\
(\mathrm{nm})\end{array}$ & $\begin{array}{c}\mathbf{I}_{\text {back mirror }} \\
(\mathrm{m} \mathrm{A})\end{array}$ & $\begin{array}{c}\mathbf{I}_{\text {front mirror }} \\
(\mathrm{mA})\end{array}$ & $\begin{array}{c}\text { Wavelength } \\
(\mathrm{nm})\end{array}$ & $\begin{array}{c}\mathbf{I}_{\text {back mirror }} \\
(\mathrm{m} \mathrm{A})\end{array}$ & $\begin{array}{c}\mathbf{I}_{\text {front mirror }} \\
(\mathrm{m} \mathrm{A})\end{array}$ \\
\hline 1B & 1527.235 & 12.5 & 2 & 1523.040 & 60 & 13.75 \\
\hline 2B & 1532.705 & 12.5 & 3.125 & 1528.495 & 60 & 17.75 \\
\hline 3B & 1537.915 & 12.5 & 4.625 & 1533.970 & 60 & 23 \\
\hline 4B & 1543.470 & 12.5 & 6.5 & 1539.800 & 60 & 30 \\
\hline 5B & 1549.065 & 12.5 & 8.75 & 1545.485 & 56 & 35 \\
\hline 6B & 1555.010 & 12.375 & 12.5 & 1552.030 & 41.5 & 35 \\
\hline 7B* & 1561.295 & 8.125 & 12.5 & 1558.495 & 31.33 & 35.83 \\
\hline 8B & 1567.830 & 5.75 & 12.5 & 1564.840 & 23.5 & 35 \\
\hline
\end{tabular}

Table 4.4: Characterizes C-Band at the start and end points of the paths along the normal twodimensional characterization. Path 7 is extended slightly to give full wavelength coverage. Path 8 will be omitted because of poor side mode suppression.

Combining paths $1 \mathrm{~A}-8 \mathrm{~A}$ with paths $1 \mathrm{~B}-8 \mathrm{~B}$ gives full paths $1-8$ (e.g. path $3 \mathrm{~A}+$ path $3 \mathrm{~B}=$ path 3$)$. For the L-band laser, all the paths overlap in wavelength except for at the end of path $8 \mathrm{~B}$ and the start of path $7 \mathrm{~A}$. So, path $8 \mathrm{~B}$ was extended by $0.8 \mathrm{~mA}$ front mirror current giving full L-band wavelength coverage from $1560.735 \mathrm{~nm}$ to 1609.920 $\mathrm{nm}$ or $\Delta \lambda=49.185 \mathrm{~nm}$. For the C-band laser, path 8 is redundant with the L-band laser's path 1. As seen on Figure (4.5) and Figure (4.7), path 8 is poorly defined compared to the other paths 1-7. This gives path 8 the worst side mode suppression of the eight paths. So the C-band laser's path 8 was omitted in favor of the L-band laser's path 1. Also, the end of path 7B and the start of path 6A do not overlap. So path 7B was extended by 0.83 $\mathrm{mA}$ front mirror current to give full wavelength coverage from $1523.040 \mathrm{~nm}$ to 1564.240 $\mathrm{nm}$ or $\Delta \lambda=41.2 \mathrm{~nm}$, with path 8 omitted.

With the start and end points of the paths needed for full wavelength coverage properly described, the next step is creating a list of points to follow along these paths. At each point in this list, the phase current will be adjusted to optimize the side mode suppression in the output signal. This process will be outlined in the next section. 
Shane O'Connor previously choose points along each path in $0.1 \mathrm{~nm}$ steps [2]. Figure (4.8) shows how the quadratic change in output wavelength for a linear change in front mirror current. The curves have a small quadratic component that will be ignored. This means that the characterization points that will be chosen along each path will be equally spaced in current instead of equally spaced in wavelength as was Shane O'Connor's focus [2]. By choosing linear jumps in current, the wavelength steps will start off larger than $0.1 \mathrm{~nm}$ and finish smaller than $0.1 \mathrm{~nm}$ at the end of each path. However, the wavelength step size will average out to $0.1 \mathrm{~nm}$. The linear steps in current will be changed to linear steps in frequency during interpolation in Section 4.3

The system, shown in Figure (4.9), uses voltage sources and current limiting resistors instead of current sources. 


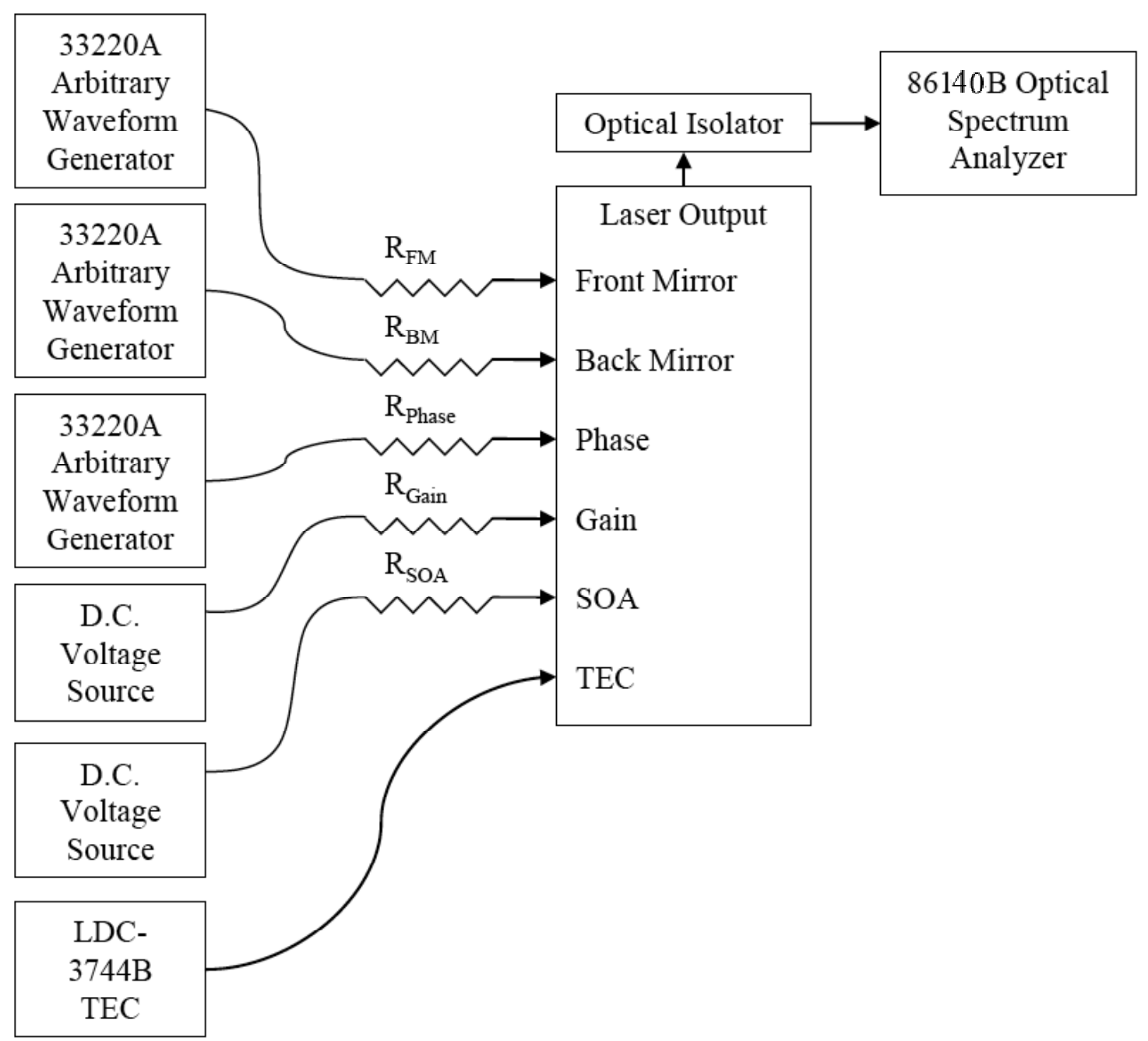

Figure 4.9: Block diagram of SG-DBR control using voltage sources and current limiting resistors instead of current sources. Three Arbitrary Waveform Generators (ARBs) are used with current limiting resistors to control the front mirror, back mirror, and phase sections. Gain and SOA sections, also with current limiting resistors, are powered by D.C. voltage sources. The SG-DBR laser is actively cooled by a thermoelectric cooler (TEC). An optical isolator is used to attenuate reflected light.

First, the start and stop currents are converted to voltages by dividing by the current limiting resistor value for each section. A multimeter will be used in Section 4.2 to calculate the voltage in each resistor.

$$
V=\frac{I}{R}
$$


Table (4.5) shows the values of the current limiting resistors. Table (4.6), Table (4.7), Table (4.8), and Table (4.9) are converted to voltages in this way from Table (4.1), Table (4.2), Table (4.3), and Table (4.4) respectively. Next, the number of characterization points per path is found by Equation (4.2), where the ceiling function rounds the contents of the parentheses up to the nearest integer and $\Delta \lambda_{\text {path }}$ is the change in wavelength over a single tuning path.

$$
N=\text { ceiling }\left(\frac{\Delta \lambda_{\text {path }}}{0.1 n m}\right)
$$

Finally the Equation (4.3) finds the voltage step size for the front mirror and back mirror voltage sources. Vstop and Vstart are the parameters for each path shown in Table (4.6), Table (4.7), Table (4.8), and Table (4.9). $\mathrm{N}$ is found from Equation (4.2).

$$
\Delta V_{\text {path }}=\frac{V_{\text {stop }}-V_{\text {start }}}{N}
$$

Table (4.10) and Table (4.11) compile this information for the L-band and C-band lasers respectively.

\begin{tabular}{|c|c|c|c|c|}
\hline & L-Band FM & L-Band BM & C-Band FM & C-Band BM \\
\hline Resistor Values & $116.2 \Omega$ & $75 \Omega$ & $121 \Omega$ & $76 \Omega$ \\
\hline
\end{tabular}

Table 4.5: Values of current limiting resistors used to convert current limits of paths to voltage limits. 


\begin{tabular}{|c|c|c|c|c|c|c|}
\hline \multirow{2}{*}{$\begin{array}{c}\text { Path } \\
\text { Number }\end{array}$} & \multicolumn{3}{|c|}{ Beginning of Path } & \multicolumn{3}{|c|}{ End of Path } \\
\hline & $\begin{array}{c}\text { Wavelength } \\
\text { (nm) }\end{array}$ & $\begin{array}{c}\mathbf{V}_{\text {back mirror }} \\
\text { (V) }\end{array}$ & $\begin{array}{l}V_{\text {front mirror }} \\
\text { (V) }\end{array}$ & $\begin{array}{c}\text { Wavelength } \\
\text { (nm) }\end{array}$ & $\begin{array}{c}\text { V }_{\text {back mirror }} \\
\text { (V) }\end{array}$ & $\begin{array}{c}V_{\text {front mirror }} \\
\text { (V) }\end{array}$ \\
\hline $\mathbf{1 A}$ & 1566.520 & 0.4500 & $\mathbf{0}$ & 1565.060 & 0.9375 & 0.1162 \\
\hline $2 \mathrm{~A}$ & 1572.825 & 0.2813 & $\mathbf{0}$ & 1570.800 & 0.9375 & 0.2034 \\
\hline $\mathbf{3 A}$ & $\mathbf{1 5 7 9 . 1 7 5}$ & 0.1594 & $\mathbf{0}$ & 1576.225 & 0.9375 & 0.3341 \\
\hline $4 \mathrm{~A}$ & 1585.955 & 0.0656 & $\mathbf{0}$ & 1582.065 & 0.9375 & 0.5084 \\
\hline $5 \mathbf{A}$ & 1592.440 & $\mathbf{0}$ & $\mathbf{0}$ & 1587.930 & 0.9375 & 0.7553 \\
\hline $6 A$ & 1598.375 & $\mathbf{0}$ & 0.0581 & 1593.840 & 0.9375 & 1.0894 \\
\hline $7 \mathrm{~A}$ & 1603.930 & $\mathbf{0}$ & 0.1888 & 1599.795 & 0.8531 & 1.4525 \\
\hline $\mathbf{8 A}$ & 1609.920 & $\mathbf{0}$ & 0.3196 & 1606.740 & 0.5719 & 1.4525 \\
\hline
\end{tabular}

Table 4.6: Converts Table (4.1) to voltages from currents by dividing by the appropriate current limiting resistor for the L-band laser.

\begin{tabular}{|c|c|c|c|c|c|c|}
\hline \multirow{2}{*}{$\begin{array}{c}\text { Path } \\
\text { Number }\end{array}$} & \multicolumn{3}{|c|}{ Beginning of Path } & \multicolumn{3}{|c|}{ End of Path } \\
\hline & $\begin{array}{l}\text { Wavelength } \\
\text { (nm) }\end{array}$ & $\begin{array}{l}V_{\text {back mirror }} \\
\text { (V) }\end{array}$ & $\begin{array}{l}\mathbf{V}_{\text {front mirror }} \\
\text { (V) }\end{array}$ & $\begin{array}{l}\text { Wavelength } \\
\text { (nm) }\end{array}$ & $\begin{array}{l}\mathbf{V}_{\text {back mirror }} \\
\text { (V) }\end{array}$ & $\begin{array}{l}V_{\text {front mirror }} \\
\text { (V) }\end{array}$ \\
\hline 1B & 1565.060 & 0.9375 & 0.1162 & 1560.735 & 4.5000 & 1.1039 \\
\hline $2 \mathrm{~B}$ & 1570.800 & 0.9375 & 0.2034 & 1566.460 & 4.5000 & 1.4525 \\
\hline 3B & 1576.225 & 0.9375 & 0.3341 & 1572.235 & 4.5000 & 1.8592 \\
\hline 4B & 1582.065 & 0.9375 & 0.5084 & 1578.035 & 4.5000 & 2.5564 \\
\hline 5B & 1587.930 & 0.9375 & 0.7553 & 1583.880 & 4.5000 & 3.3698 \\
\hline $6 \mathrm{~B}$ & 1593.840 & 0.9375 & 1.0894 & 1590.215 & 4.0125 & 4.0670 \\
\hline $7 \mathrm{~B}$ & 1599.795 & 0.8531 & 1.4525 & 1597.080 & 3.0000 & 4.0670 \\
\hline $8 \mathrm{~B}^{*}$ & 1606.740 & 0.5719 & 1.4525 & 1604.080 & 2.2320 & 4.1600 \\
\hline
\end{tabular}

Table 4.7: Converts Table (4.2) to voltages from currents by dividing by the appropriate current limiting resistor for the L-band laser.

\begin{tabular}{|c|c|c|c|c|c|c|}
\hline \multirow{2}{*}{$\begin{array}{c}\text { Path } \\
\text { Number }\end{array}$} & \multicolumn{3}{|c|}{ Beginning of Path } & \multicolumn{3}{|c|}{ End of Path } \\
\hline & $\begin{array}{c}\text { Wavelength } \\
\text { (nm) }\end{array}$ & $\begin{array}{l}\mathrm{V}_{\text {back mirror }} \\
\text { (V) }\end{array}$ & $\begin{array}{l}V_{\text {front mirror }} \\
\text { (V) }\end{array}$ & $\begin{array}{l}\text { Wavelength } \\
\text { (nm) }\end{array}$ & $\begin{array}{l}\mathrm{V}_{\text {back mirror }} \\
\text { (V) }\end{array}$ & $\begin{array}{l}\mathbf{V}_{\text {front mirror }} \\
\text { (V) }\end{array}$ \\
\hline$\overline{1 A}$ & 1529.130 & 0.3230 & $\mathbf{0}$ & 1527.235 & 0.9500 & 0.2420 \\
\hline $2 \mathrm{~A}$ & 1535.165 & 0.1900 & $\mathbf{0}$ & 1532.705 & 0.9500 & 0.3781 \\
\hline$\overline{\mathbf{3 A}}$ & 1541.265 & 0.0760 & $\mathbf{0}$ & 1537.915 & 0.9500 & 0.5596 \\
\hline $\mathbf{4 A}$ & 1547.330 & $\mathbf{0}$ & 0.0151 & 1543.470 & 0.9500 & 0.7865 \\
\hline $5 \mathrm{AA}$ & 1552.950 & $\mathbf{0}$ & 0.0908 & 1549.065 & 0.9500 & 1.0588 \\
\hline $6 \mathrm{~A}$ & 1558.585 & $\mathbf{0}$ & 0.2118 & 1555.010 & 0.9405 & 1.5125 \\
\hline$\overline{7 A}$ & 1564.240 & $\mathbf{0}$ & 0.3933 & 1561.295 & 0.6175 & 1.5125 \\
\hline$\overline{8 A}$ & 1569.970 & $\mathbf{0}$ & 0.5596 & 1567.830 & 0.4370 & 1.5125 \\
\hline
\end{tabular}

Table 4.8: Converts Table (4.3) to voltages from currents by dividing by the appropriate current limiting resistor for the $\mathrm{C}$-band laser. 


\begin{tabular}{|c|c|c|c|c|c|c|}
\hline \multirow{2}{*}{$\begin{array}{c}\text { Path } \\
\text { Number }\end{array}$} & \multicolumn{3}{|c|}{ Beginning of Path } & \multicolumn{3}{|c|}{ End of Path } \\
\hline & $\begin{array}{c}\text { Wavelength } \\
\text { (nm) }\end{array}$ & $\begin{array}{l}V_{\text {back mirror }} \\
\text { (V) }\end{array}$ & $\begin{array}{l}\mathbf{V}_{\text {front mirror }} \\
\text { (V) }\end{array}$ & $\begin{array}{c}\text { Wavelength } \\
\text { (nm) }\end{array}$ & $\begin{array}{l}V_{\text {back mirror }} \\
\text { (V) }\end{array}$ & $\begin{array}{l}\mathbf{V}_{\text {front mirror }} \\
\text { (V) }\end{array}$ \\
\hline 1B & 1527.235 & 0.9500 & 0.2420 & 1523.040 & 4.5600 & 1.6638 \\
\hline $2 \mathrm{~B}$ & 1532.705 & 0.9500 & 0.3781 & 1528.495 & 4.5600 & 2.1478 \\
\hline 3B & 1537.915 & 0.9500 & 0.5596 & 1533.970 & 4.5600 & 2.7830 \\
\hline 4B & 1543.470 & 0.9500 & 0.7865 & 1539.800 & 4.5600 & 3.6300 \\
\hline 5B & 1549.065 & 0.9500 & 1.0588 & 1545.485 & 4.2560 & 4.2350 \\
\hline 6B & 1555.010 & 0.9405 & 1.5125 & 1552.030 & 3.1540 & 4.2350 \\
\hline $7 B^{*}$ & 1561.295 & 0.6175 & 1.5125 & 1558.495 & 2.3811 & 4.3354 \\
\hline 8B & 1567.830 & 0.4370 & 1.5125 & 1564.840 & 1.7860 & 4.2350 \\
\hline
\end{tabular}

Table 4.9: Converts Table (4.4) to voltages from currents by dividing by the appropriate current limiting resistor for the $\mathrm{C}$-band laser.

\begin{tabular}{|c|c|c|c|c|c|c|c|}
\hline $\begin{array}{c}\text { Path } \\
\text { Number }\end{array}$ & $\begin{array}{c}\text { Number } \\
\text { of Points }\end{array}$ & $\begin{array}{c}\text { FM Step } \\
\text { Size }(\mathbf{m V})\end{array}$ & $\begin{array}{c}\text { BM Step } \\
\text { Size }(\mathbf{m V})\end{array}$ & $\begin{array}{c}\text { Path } \\
\text { Number }\end{array}$ & $\begin{array}{c}\text { Number } \\
\text { of Points }\end{array}$ & $\begin{array}{c}\text { FM Step } \\
\text { Size (mV) }\end{array}$ & $\begin{array}{c}\text { BM Step } \\
\text { Size (mV) }\end{array}$ \\
\hline 1A & 15 & 7.75 & 32.50 & 1B & 44 & 22.45 & 80.97 \\
\hline 2A & 21 & 9.68 & 31.25 & 2B & 40 & 31.23 & 89.06 \\
\hline 3A & 30 & 11.14 & 25.94 & 3B & 40 & 38.13 & 89.06 \\
\hline 4A & 39 & 13.04 & 22.36 & 4B & 41 & 49.95 & 86.89 \\
\hline 5A & 46 & 16.42 & 20.38 & 5B & 41 & 63.77 & 86.89 \\
\hline 6A & 46 & 22.42 & 20.38 & 6B & 37 & 80.48 & 83.11 \\
\hline 7A & 42 & 30.09 & 20.31 & 7B & 28 & 93.38 & 76.67 \\
\hline 8A & 32 & 35.40 & 17.87 & 8B & 28 & 93.38 & 57.25 \\
\hline
\end{tabular}

Table 4.10: L-band path data used for characterization purposes.

\begin{tabular}{|c|c|c|c|c|c|c|c|}
\hline $\begin{array}{c}\text { Path } \\
\text { Number }\end{array}$ & $\begin{array}{l}\text { Number } \\
\text { of Points }\end{array}$ & $\begin{array}{c}\text { FM Step } \\
\text { Size }(\mathrm{mV}) \\
\end{array}$ & $\begin{array}{c}\text { BM Step } \\
\text { Size (mV) }\end{array}$ & $\begin{array}{c}\text { Path } \\
\text { Number }\end{array}$ & $\begin{array}{l}\text { Number } \\
\text { of Points }\end{array}$ & $\begin{array}{c}\text { FM Step } \\
\text { Size }(m V)\end{array}$ & $\begin{array}{c}\text { BM Step } \\
\text { Size }(\mathrm{mV})\end{array}$ \\
\hline $1 \mathrm{~A}$ & 19 & 12.74 & 33.00 & 1B & 42 & 33.85 & 85.95 \\
\hline $2 \mathrm{~A}$ & 25 & 15.13 & 30.40 & $2 B$ & 43 & 41.15 & 83.95 \\
\hline 3A & 34 & 16.46 & 25.71 & 3B & 40 & 55.58 & 90.25 \\
\hline $4 \mathrm{~A}$ & 39 & 19.78 & 24.36 & 4B & 32 & 76.85 & 97.57 \\
\hline $5 \mathrm{~A}$ & 39 & 24.82 & 24.36 & 5B & 36 & 88.23 & 91.83 \\
\hline 6A & 36 & 36.13 & 26.13 & 6B & 30 & 90.75 & 73.78 \\
\hline $7 \mathrm{~A}$ & 30 & 37.31 & 20.58 & $7 B$ & 27 & 100.83 & 62.98 \\
\hline
\end{tabular}

Table 4.11: C-band path data used for characterization purposes.

Table (4.6), Table (4.7), Table (4.8), Table (4.9), Table (4.10), and Table (4.11) are used for the three-dimensional characterization process. 


\subsection{Three-Dimensional Characterization}

Now that the L-band and C-band lasers are characterized two-dimensionally and the paths are properly formed, characterization of the phase section can begin. This is called three-dimensional characterization.

To construct a linear continuous ramp in frequency or wavelength, two requirements exist. First, currents in the front mirror and back mirror sections are needed that follow the tuning paths and were previously discussed in the two-dimensional characterization section. Secondly, to achieve full continuity in the ramp, a continuous injection current must also be applied to the phase input of the laser. Simultaneously increasing the injection current on the front mirror, back mirror, and phase sections continuously will increase the frequency or decrease the wavelength continuously [2].

Shane O'Connor previously opted to assure the steps along the tuning paths were linear in wavelength (refer back to Figure (4.8) to see the nonlinearities in the paths). As stated before, this procedure prefers to ignore the quadratic nature of the wavelength versus input current and deal with this nonlinearity later during interpolation.

As before, Shane O'Connor's LabVIEW program is used [2]. However, the program was modified slightly to automatically jump along the set tuning paths in the chosen step size shown in Table (4.10) and Table (4.11). The three-dimensional characterization program works in conjunction with the test setup described in the block diagram in Figure (4.10). LabVIEW controls the Agilent 33220A Arbitrary Waveform Generators (ARB) voltage sources, records the voltages off the ARBs at each data point, and records the wavelength and output power at each data point. An Agilent 86140B 
Optical Spectrum Analyzer (OSA) computes the wavelength and output power data as well as provides the necessary waveform display to view the side modes.

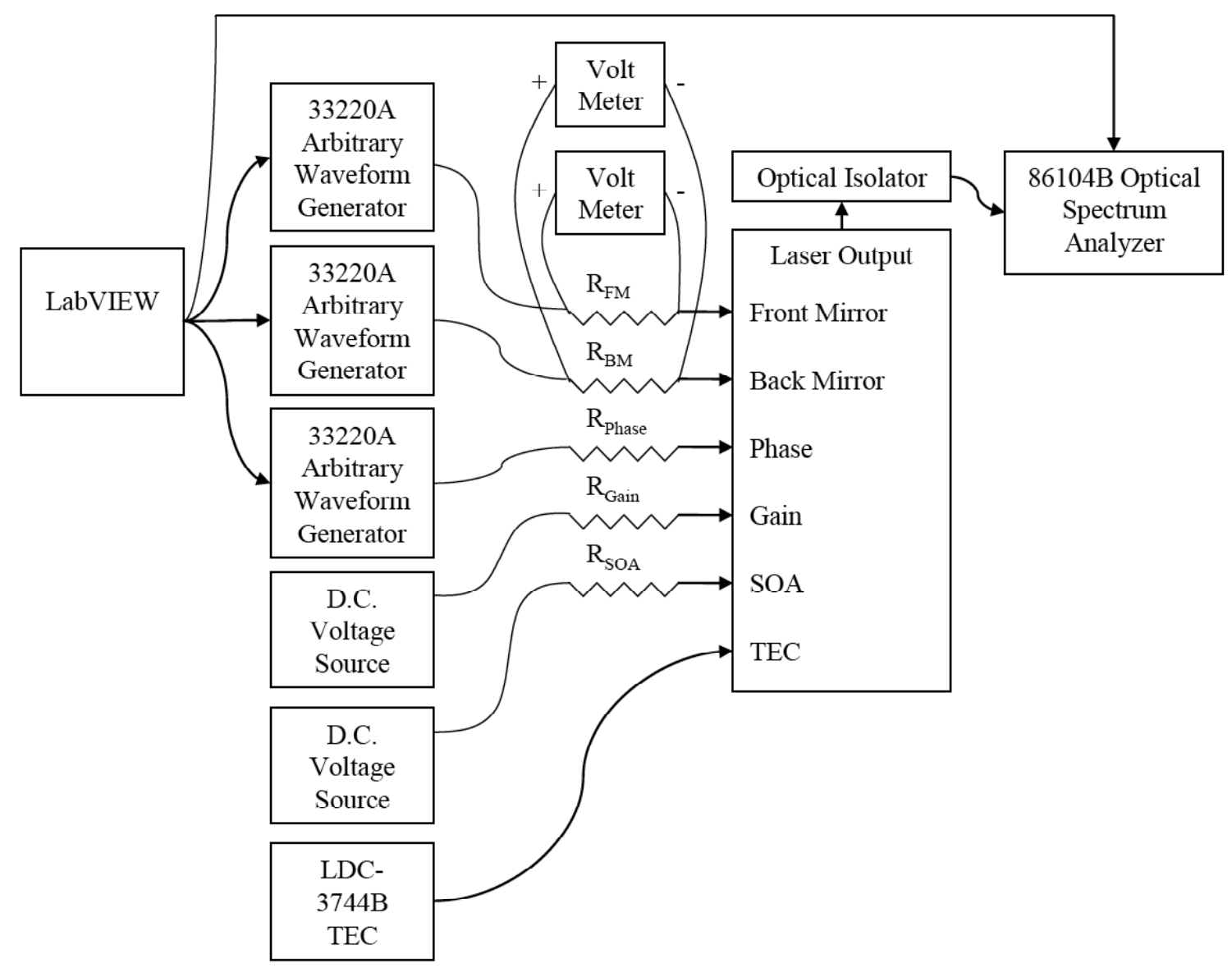

Figure 4.10: Block diagram of three-dimensional characterization test setup. The LabVIEW program controls the output voltage of the three ARBs. The program also records the ARB voltages for the front mirror, back mirror, and phase with the laser output power and wavelength. The two volt meters are needed since the voltages found earlier in Table (4.6), Table (4.7), Table (4.8), Table

(4.9), Table (4.10), and Table (4.11) are given across the current limiting resistors. D.C. voltage sources bias the gain and $S O A$ sections at $100 \mathrm{~mA}$ and $150 \mathrm{~mA}$ respectfully [2].

Creating wavelength tuning ramps along the paths described by Table (4.6), Table (4.7), Table (4.8), Table (4.9), Table (4.10), and Table (4.11) requires several steps. The first step is to recognize that since the voltages that were calculated from the currents into each section were found by dividing by each current limiting resistor, these voltages must be measured across each resistor and not at the ARB input. This means attaching 
voltmeters across the front mirror and back mirror current limiting resistors. The phase section also receives current from the ARB in the same way.

It is helpful to next calculate, in a Microsoft Excel spreadsheet, the series of ARB voltages necessary to follow each path from each start point to each ending point by stepping in the predetermined voltage step sizes shown in Table (4.10) and Table (4.11). Each of these points will need to be optimized to give the maximum side mode suppression. Image "b" in Figure (4.11) shows a bias point with maximum side mode suppression.

At each characterization point along the paths, the phase ARB voltage must be incremented up until a suitable bias point is found that ideally suppresses the side modes. Figure (4.11) demonstrates two separate bias points, one improperly chosen, and one properly chosen. Each side mode originates from the adjacent cavity modes, which were described in Section 2.5. Therefore the ideal phase voltage for each bias point selects a wavelength that corresponds with a cavity mode in the laser. Refer back to Section 2.6 for more information.

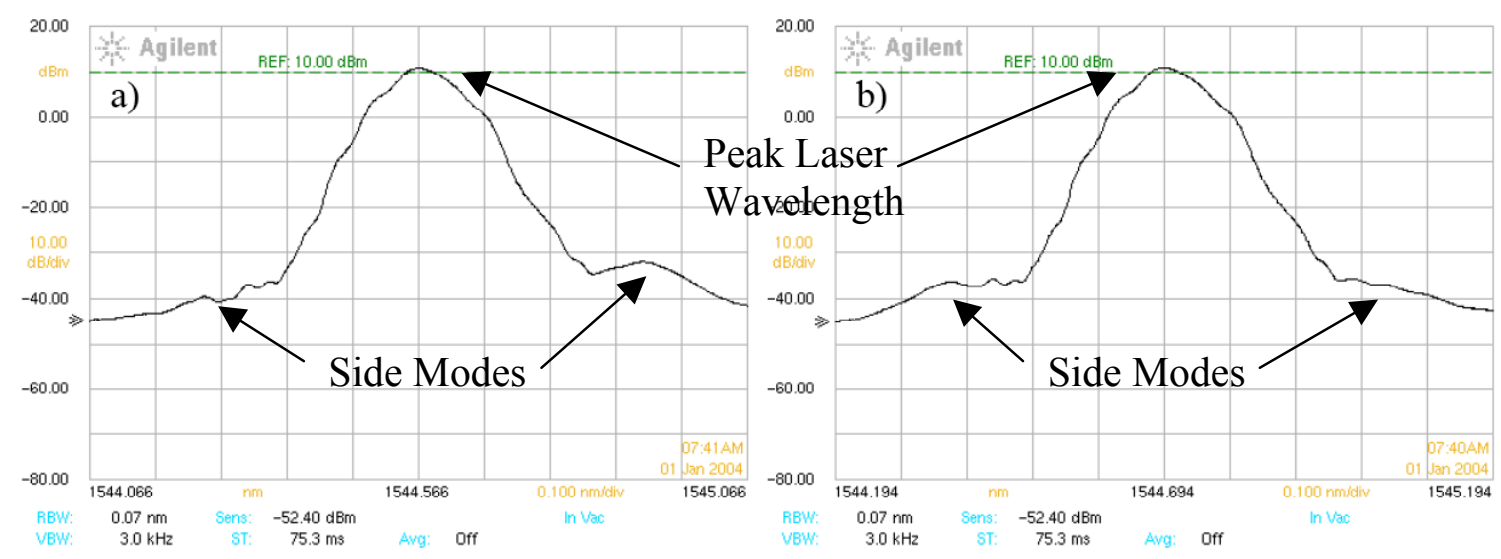

Figure 4.11: Two separate bias points for the same characterization point. Image a) demonstrates a non-ideal suppression of the side modes. Note how the high wavelength $0.3 \mathrm{~nm}$ side mode has higher power than the low wavelength $0.3 \mathrm{~nm}$ side mode. This means the side modes are not optimally suppressed. Image b) exhibits an ideal suppression of the side modes. Note how the low and high wavelength side modes have equal power levels and are optimally suppressed. [2] 
After the characterization point is successfully optimized, the same procedure is repeated for each point along each path described by Table (4.6), Table (4.7), Table (4.8), Table (4.9), Table (4.10), and Table (4.11). However, the ARB voltage has a finite range (maximum 5 V D.C.), as does the input current into the phase section $(20 \mathrm{~mA})$. As the ARB voltage increases and the phase section current becomes limited by the $20 \mathrm{~mA}$ maximum value, one must find a phase voltage near $0 \mathrm{~V}$ (commonly between $0.34 \mathrm{~V}$ and $0.7 \mathrm{~V}$ ) that exactly matches the wavelength at the last point before the phase current was limited. Only then can a new continuous tuning segment be appended to the previous continuous tuning segment. This process is known as "stitching."

Stitching continuous tuning segments together leaves the frequency ramp vulnerable to stitching errors. A stitching error occurs when the last frequency of one continuous segment does not match the first frequency of the next tuning segment. Chapter 7 discusses the sources of, methods for decreasing, and implications of stitching errors. Figure (4.12) shows a stitching error that repeats frequency coverage and another that omits frequency coverage.
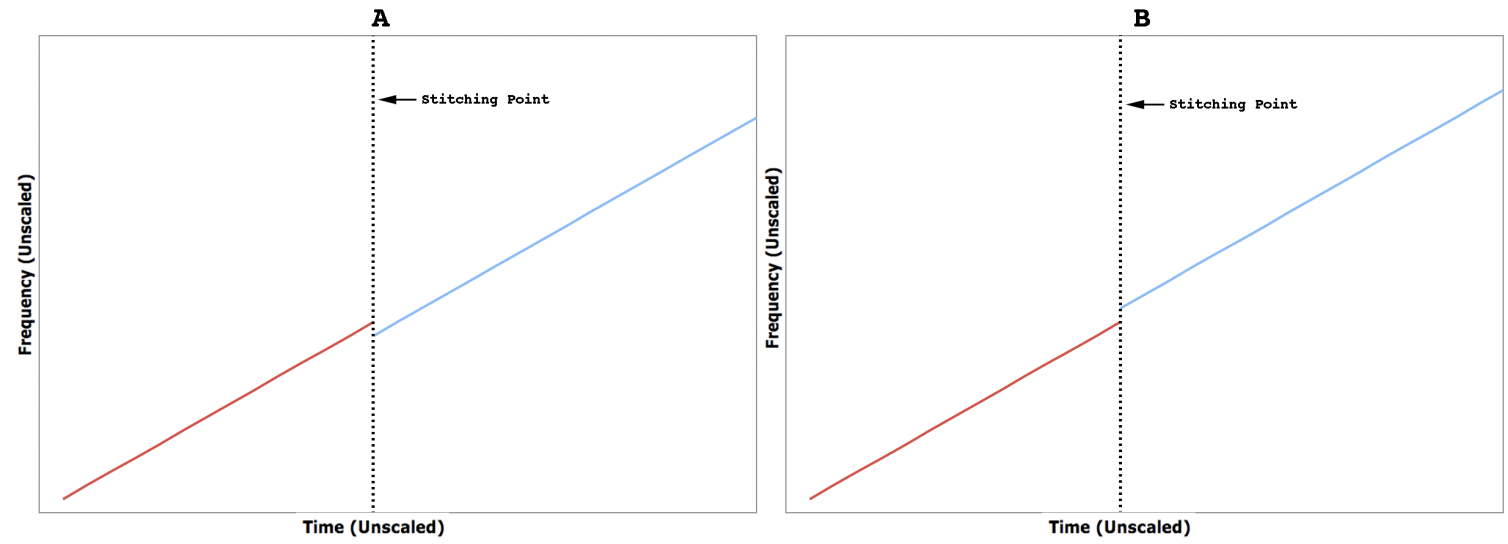

Figure 4.12: Demonstration of two types of stitching errors. The red frequency ramp represents the first continuous tuning segment while the blue frequency ramp stands for the second. The stitching error in image " $A$ " repeats frequency coverage to the frequency ramp. The stitching error in image " $B$ " omits frequency coverage to the frequency ramp. 
To characterize each laser at points approximately every $0.1 \mathrm{~nm}$ results in 299 points to be characterized for the L-band laser and 250 points for the C-band laser. Since the process is not automated, the time to three dimensionally characterize a point varies. However, after some practice, about 90 seconds of time per point can be expected. This relates to approximately 7.5 hours of characterization time for the L-band laser and approximately 6.25 hours for the C-band laser.

Stitching becomes one of the largest sources of error in the laser. Not only does the switching times of the ARB cause a $50 \mathrm{~ns}$ time after a stitch where the output frequency is undetermined, but after a stitch point, a host of potential measurement errors will cause the new path not start off exactly where the last path ended. Stitching errors will be analyzed in further in Chapter 7.

After each point along each path described by Table (4.6), Table (4.7), Table (4.8), Table (4.9), Table (4.10), and Table (4.11) is successfully characterized and recorded, the output from the LabVIEW program gives path characterization files. Each file includes the three ARB voltages (FM, BM, and phase), the wavelength of the peak signal, and the power level of that peak for each characterization point. Since each characterization point is approximately $100 \mathrm{pm}$ apart from adjacent points, interpolation is needed to reduce this to $1 \mathrm{pm}$ or less.

\subsection{Interpolation}

Previous work by Shane O'Connor focused on creating a linear wavelength ramp from the SG-DBR laser. While this was a large undertaking and a great leap forward, OCT and other types of optical testing require linear frequency ramps instead of linear 
wavelength ramps. The following series of equations demonstrates why a constant change in wavelength does not translate to a constant change in frequency. Start with:

$$
\lambda=\frac{c}{f}
$$

find the difference between two wavelengths:

$$
\lambda_{2}-\lambda_{1}=\frac{c}{f_{2}}-\frac{c}{f_{1}}
$$

which when simplified becomes Equation (4.6)

$$
\Delta \lambda=\frac{c \Delta f}{f_{1} f_{2}}
$$

One can see that the following term:

$$
\frac{1}{f_{1} f_{2}}
$$

causes a nonlinearity in the relationship between constant wavelength and constant frequency. Figure (4.13) shows the difference between linear frequency ramps vs. linear wavelength ramps. The maximum frequency difference between the two ramps is 47.11 GHz. The deviation from linearity during a linear wavelength ramp causes spectral broadening during OCT measurements, which results in decreased resolution, as stated in Section 3.1. 


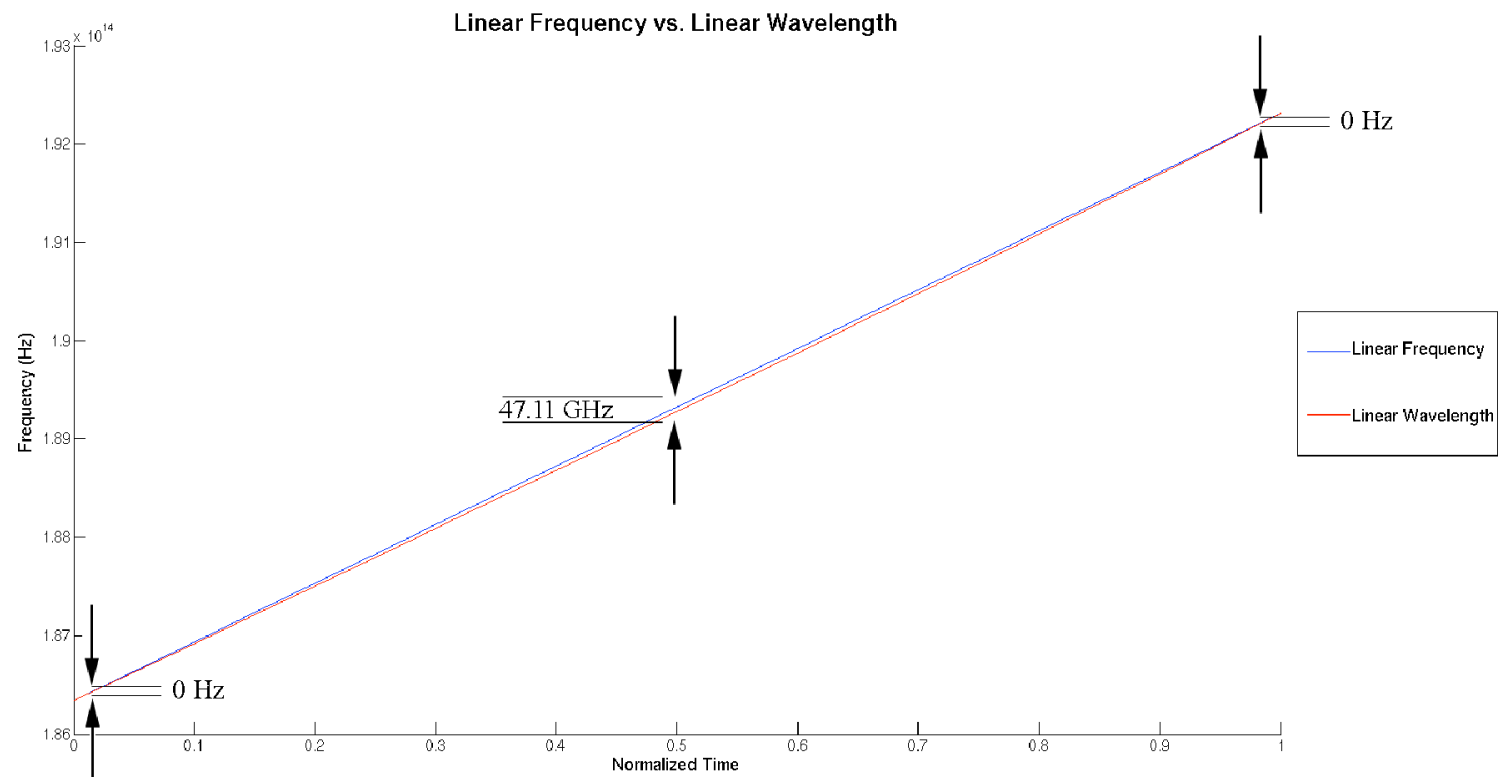

Figure 4.13: Differences due to a linear frequency ramp and a linear wavelength ramp. The linear frequency ramp is a linear frequency vs. time line while the linear frequency ramp deviates from linear, a maximum of $47.11 \mathrm{GHz}$.

To create a tuning dictionary to generate a linear wavelength ramp, the threedimensional path characterization files were interpolated at $1 \mathrm{pm}$ intervals. This was done by drawing a line between two characterization points and essentially filling in the blanks every $1 \mathrm{pm}$.

To create the needed tuning dictionary for a linear frequency ramp, a MATLAB program was created. The first step to interpolate in frequency instead of wavelength, is to convert the wavelengths in the three-dimensional characterization files to frequency by the formula:

$$
f=\frac{c}{\lambda}
$$

The linear wavelength ramp was interpolated at $1 \mathrm{pm}$ steps, so a similar resolution is needed at a convenient step size. 100 megahertz (MHz) steps, which corresponds to approximately $0.82 \mathrm{pm}$, from Equation (4.6), was chosen for simplicity and since it has 
slightly decreased spacing. Each set of points characterized for each path was then interpolated in $100 \mathrm{MHz}$ steps.

To finish creating a full linear frequency ramp, the individual linear frequency interpolated path data needs to be assembled into one continuous linear frequency ramp. If there is an overlap between frequency path data, the lower current data will be cut from the overlapping paths to decrease the chance of the ramp accidentally changing modes. Once the linear frequency ramp is assembled from the separate paths, the data needs to be modified before acceptable ARB input files can be created. The Agilent 33220A Arbitrary Waveform Generator input data needs to be between $0-1$ and then scaling is performed as the file is inputted. All input data must be first divided by $5 \mathrm{~V}$. Then, to find the scaling factor needed at the time of the ARB initialization, the equation:

$$
F=5(\max (X)-\min (X))
$$

was applied in Excel. $\mathrm{F}$ is the scaling factor and $\mathrm{X}$ is the range of data for each ARB input (i.e. front mirror, back mirror, and phase). Now a new text file needs to be created for each ARB voltage source using the scaled full linear frequency ramp data. Figure (4.14) shows the ARB waveforms for the L-band and C-band lasers. 

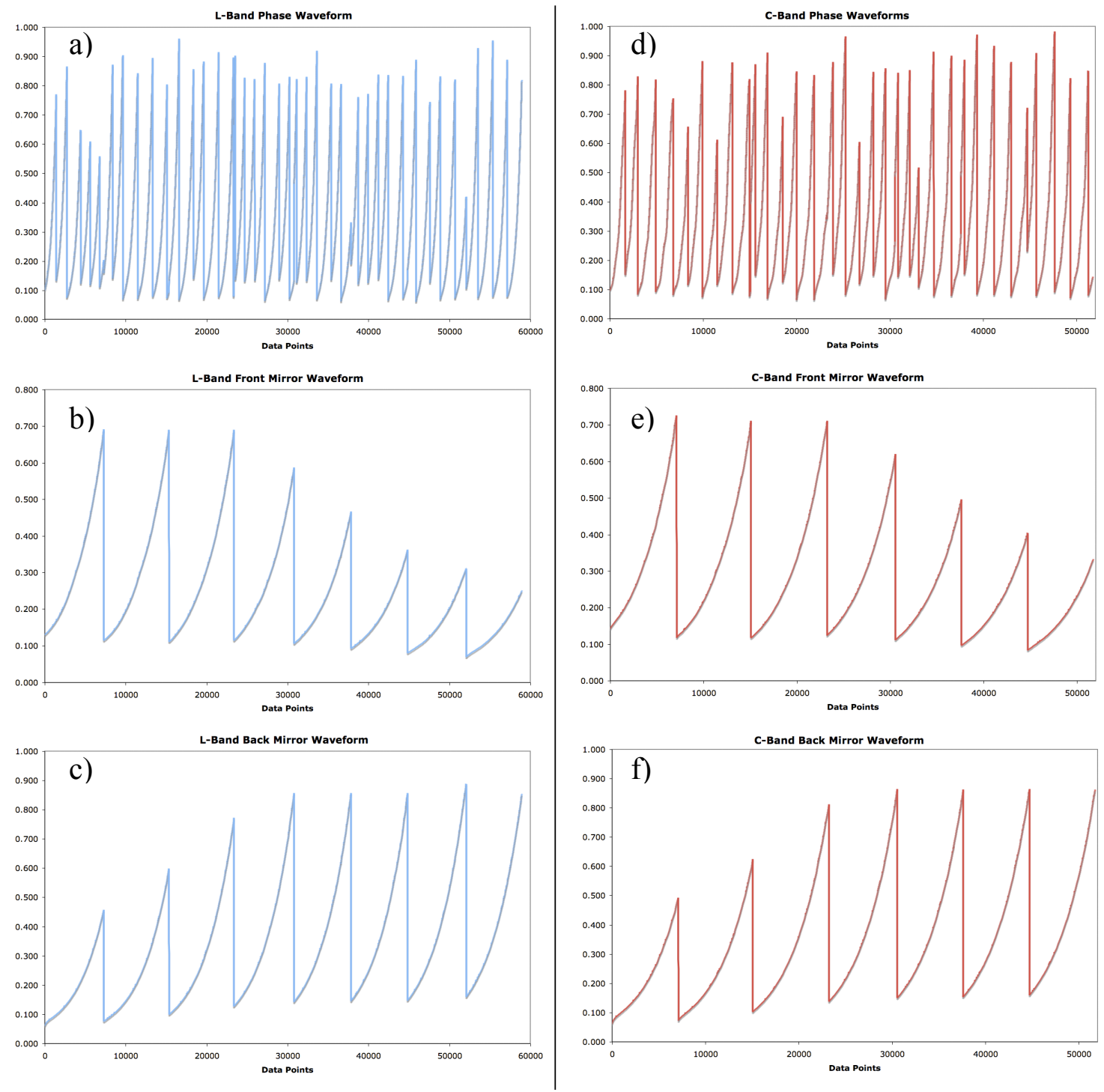

Figure 4.14: Input ARB waveforms that are used to control the linear frequency ramps for L-band and C-band lasers. Image " $a$ " is the input waveform to the L-band phase section. Image "b" is the input waveform to the $L$-band front mirror section. Image "c" is the input waveform to the $L-b a n d$ back mirror section. Image " $d$ " is the input waveform to the $C$-band phase section. Image " $e$ " is the input waveform to the $\mathrm{C}$-band front mirror section. Image "f $\mathrm{f}$ " is the input waveform to the $\mathrm{C}$-band back mirror section. Image "a", " $b$ ", and "c" are run together to create a L-band linear frequency ramp. Image " $d$ ", " $e$ ", and "f" are run together to create a $C$-band linear frequency ramp. X-axis represent the data points of each waveform while the $Y$-axis represents the normalized ARB voltage of each waveform.

\subsection{Running the SG-DBR Linear Frequency Ramp}

To implement the three frequency control waveforms in Figure (4.14) for each laser, the Agilent ARB program asks for the waveform files one at a time. As each file is inputted, the Agilent program asks for the repetition frequency and amplitude information 
associated with the laser sweep. Figure (4.15) shows a block diagram of the test setup used to run the waveforms shown in Figure (4.14) to create a linear wavelength ramp.

Figure (4.18) shows a picture of the test setups for the L-band and C-band lasers.

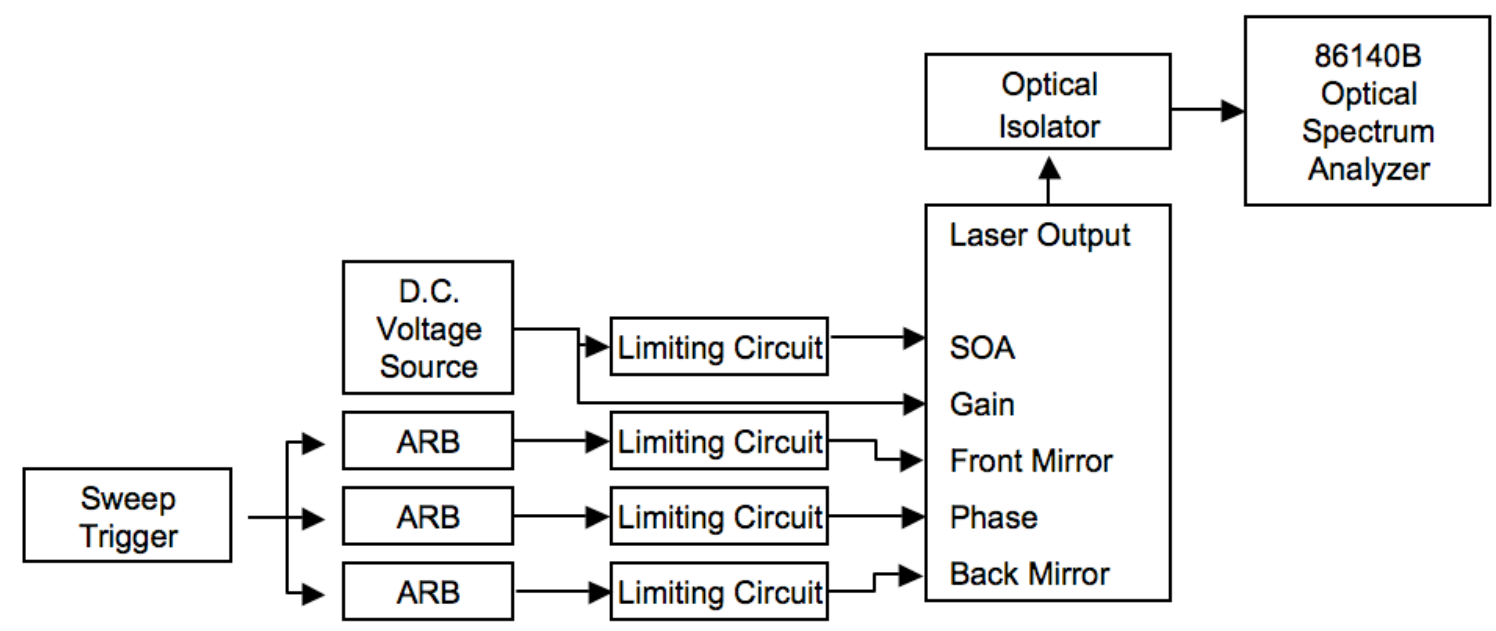

Figure 4.15: Block diagram of the test setup to run a linear frequency ramp on a L-band or C-band laser. The waveforms "a", "b", and "c" shown in Figure (4.14) are used to create a L-band linear frequency ramp. The waveforms " $d$ ", " $e$ ", and "f $f$ " shown in Figure (4.14) are used to create a C-band linear frequency ramp. Figure (4.16) shows a block diagram of the limiting circuit. Figure (4.17) shows a picture of a limiting circuit.

Figure (4.16) expands the limiting circuits in Figure (4.15), which prevent the maximum current and negative voltages from being inputted to each laser port by incorporating a current limiting resistor and a reversed biased diode with small reverse breakdown voltage. Figure (4.17) shows a picture of the circuit in Figure (4.16). 
To
ARB

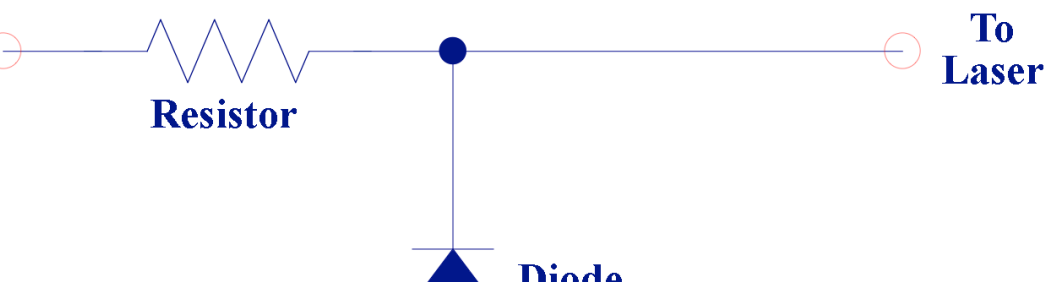

Diode

Figure 4.16: Current limiting circuit used in laser test setups to prevent overloading and negative voltages in each laser port except the gain section. When the ARB voltage is set to its maximum value, the current limiting resistor is chosen to keep the current into the laser section below the maximum value. See Figure (4.17) for a picture of one of these circuits.

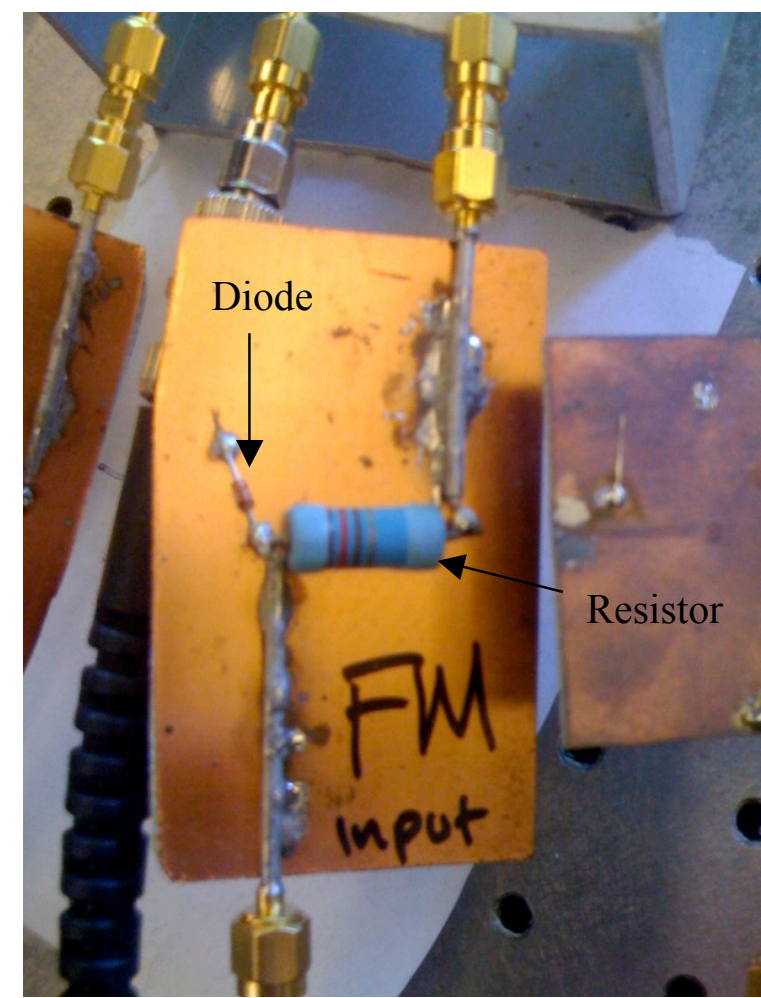

Figure 4.17: High power resistor protects the laser section from exceeding the maximum current. The low reverse breakdown voltage diode protects the laser section from negative currents. See Figure (4.16) for a schematic of this circuit. 


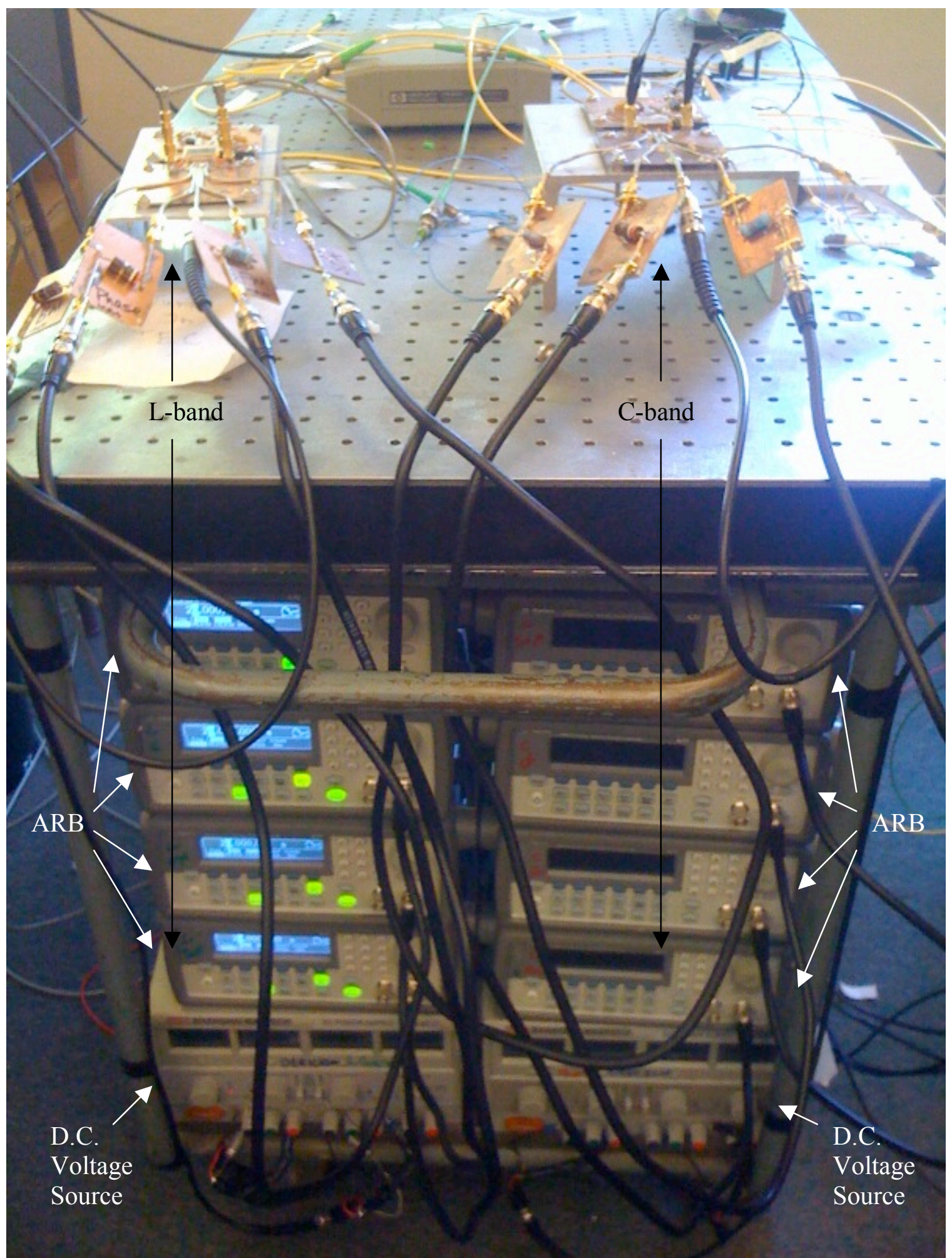

Figure 4.18: Test setups for the L-band and C-band laser. The test setup on the left side is used on the L-band laser while the right side is used for the $\mathrm{C}$-band laser. For this case, one of the ARBs in each stack is used in D.C. voltage source mode to bias the SOA of each laser. The three other ARBs are used to control the front mirror, back mirror, and phase of each laser. The L-band ARBs are "on" in the picture. 
The waveforms, shown in Figure (4.14), can be set to run at any equal repetition rate, however the performance of the frequency ramp will always be more linear at lower speeds due mostly to the effects of stitching errors. See Chapter 7 for more information on stitching errors.

Not only do the waveforms need to run at the same repetition rate, but also the three waveforms must be synchronized by applying the 1 cycle "burst" function on the ARBs. A function generator with a square wave or pulse output triggers the three synchronized waveforms. A slightly lower frequency setting is needed on the trigger signal to guarantee all waveforms are triggered simultaneously. Trigger jitter and other non-ideal effects prevent perfect waveform synchronization [2].

Figure (4.19) shows the output linear frequency ramp waveform from each laser running at slow speeds from the Agilent 86140B Optical Spectrum Analyzer. The trace is set to "MAX HOLD" which keeps the maximum value recorded in the sweep bandwidth. Note the overlap in frequency coverage between the L-band and C-band frequency ramps, which allows for the concatenation of the two frequency ramps into a single frequency ramp. Concatenation will be outlined in Chapter 6. Also note the amplitude "ripple" in which the output power depends on the frequency. Power leveling will be used to significantly reduce the amplitude ripple in each frequency ramp in Chapter 5. 


\section{Linear Frequency Ramps}

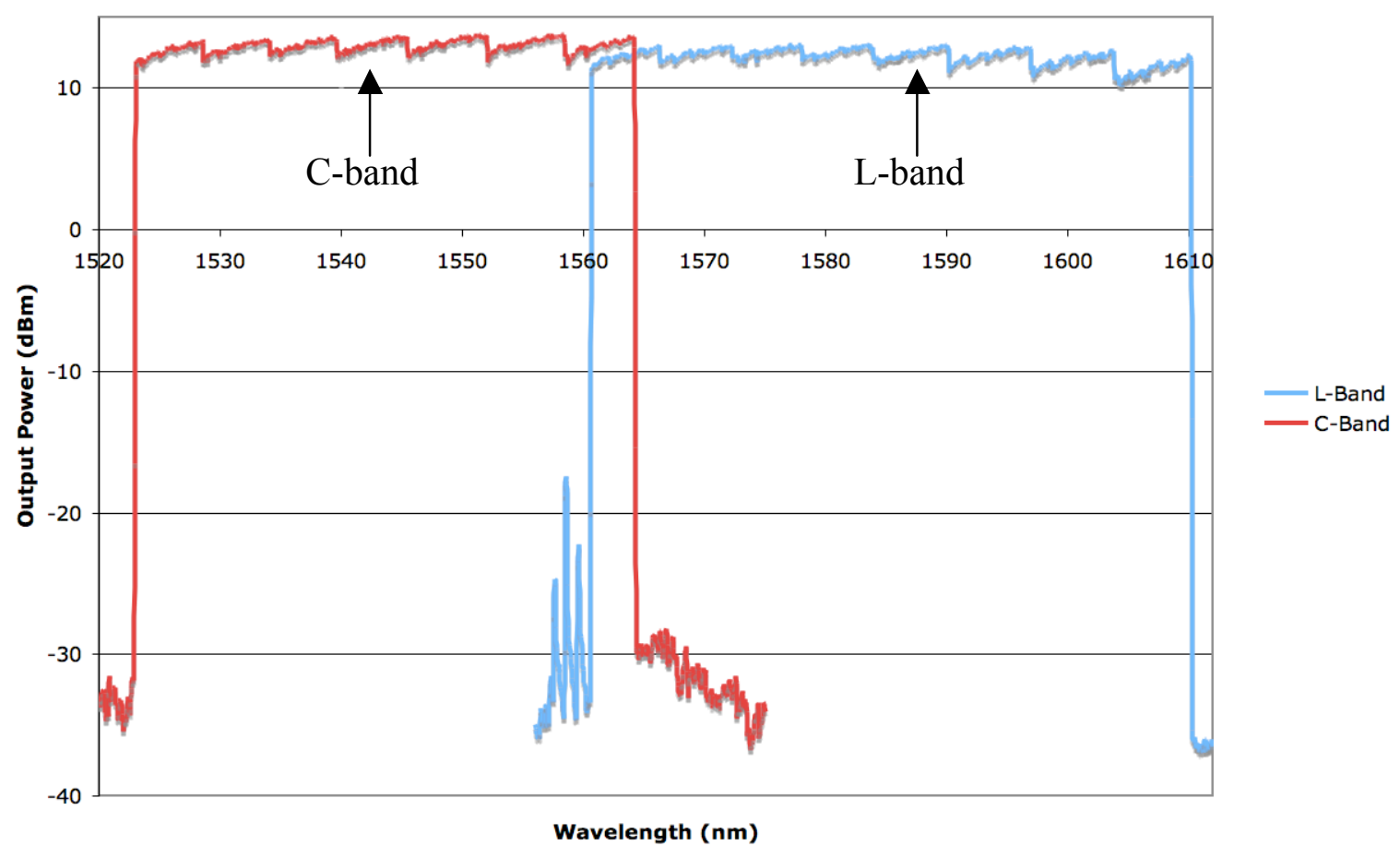

Figure 4.19: Frequency ramps of $L$-band and $C$-band lasers run at a $1 \mathrm{~Hz}$ repetition rate. Note the overlap of the L-band and C-band frequency ramps, which allow for concatenation of the two ramps into a single ramp. Also note the variation of the output power versus frequency, which will be expanded upon in the next chapter. 


\section{CHAPTER 5 - Power Calibration and Power Leveling of a L-band and C-band Laser}

Modulating the currents entering the three frequency tuning ports controls the wavelength of the SG-DBR laser. A property of a SG-DBR laser is that the output power of the laser is dependant on the input currents that tune the laser. A frequency ramp with level output power versus frequency is needed for various types of optical sensing techniques, including OCT. The SOA of the SG-DBR laser can vary the output power by modulating the input current to the section. Characterization of the SOA port, performed in Section 5.1 will relate the input current to the output power for various laser output frequencies. Once the output power versus input current relationship of the SOA is known, Section 5.2 constructs a procedure to reduce the ripple in the frequency ramp.

\section{1 - Characterization of the Semiconductor Optical Amplifier}

The output power of the laser is dependant on the input currents into the front mirror, back mirror, and phase sections. The higher the current inputted into a section, the higher the carrier density in the laser. Since the optical absorption in the laser rises when the carrier density increases, the output power of the laser decreases when the currents in each section rise.

The three-dimensional characterization in Section 4.2 recorded the amplitude data for each characterization point. This amplitude data, as well as the various ARB voltages, were interpolated in Section 4.3. Figure (5.1) plots the output power data for the L-band and C-band lasers versus frequency. Each discontinuity in the output power is directly related to a point in which the input currents to the laser jump from high to low. This occurs since a discontinuity in the current waveforms will abruptly change the attenuation inside the laser. The large discontinuities in Figure (5.1) are a result of 
stitching switching between paths, since the front mirror and back mirror currents change from high to low suddenly. The small discontinuities are due to stitching two smaller continuous tuning segments together by changing the phase current from high to low, as discussed in Section 4.2.

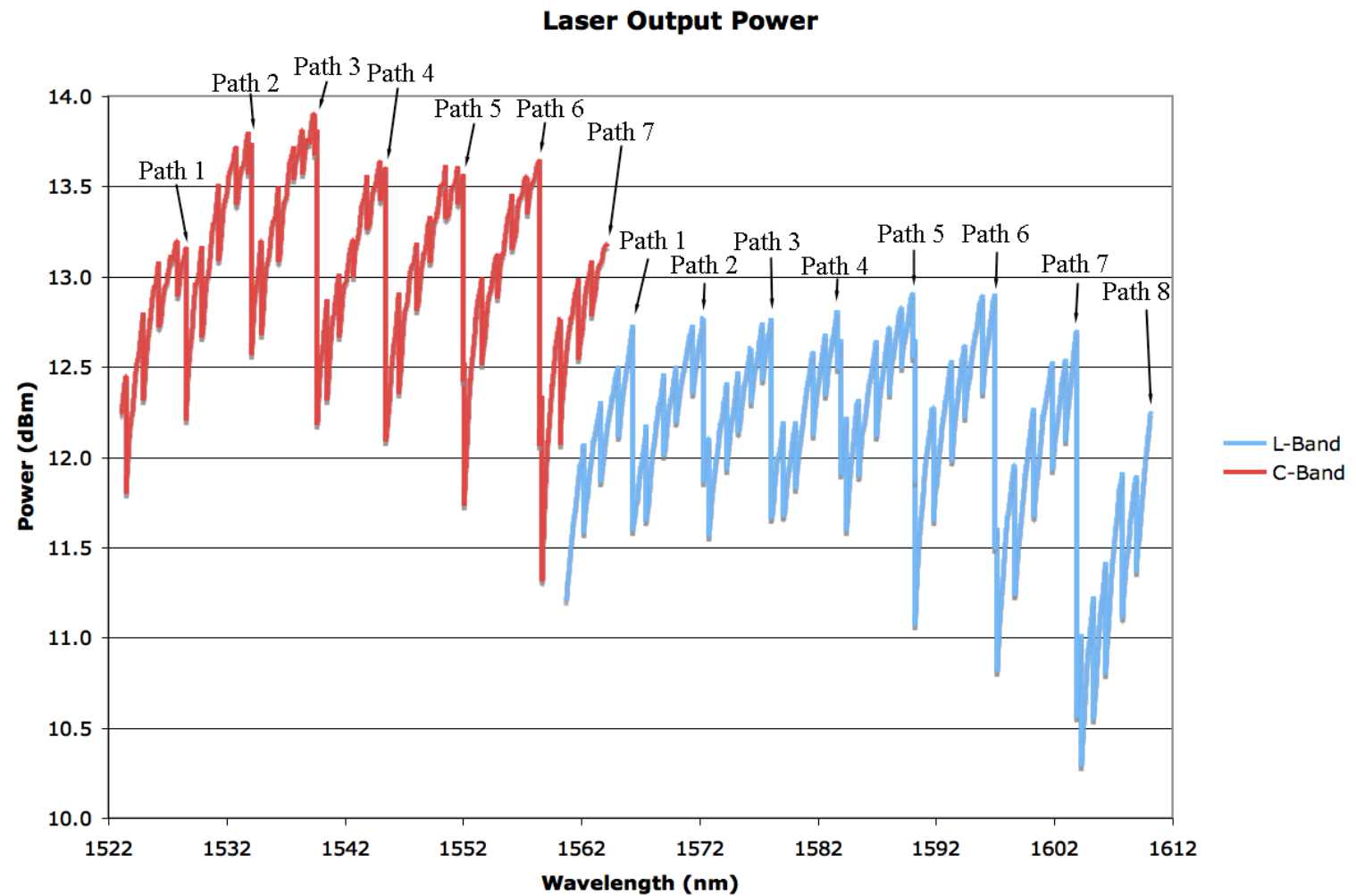

Figure 5.1: Laser output power determined from three-dimensional characterization of the two lasers. Data will be used to power level the two linear frequency ramps. Also note the start of each path for a linear frequency ramp. Note: An increasing frequency ramp results in decreasing wavelength. Large discontinuities are a result of changing the front mirror and back mirror currents from high to low. The small discontinuities are due to switching the phase current from high to low.

The SOA must be successfully characterized so that the relationship between the ARB output voltage and the laser output power as well as any frequency dependence is known. Not only does the SOA have a nonlinear input current vs. output power relationship, but also this relationship changes with respect to frequency. So to properly characterize the ARB output voltage versus the laser output power the voltage/power curves were computed at the low, middle, and high wavelength points of the two lasers. 
At stitching points there are two sets of input currents that result in the same output frequency. By characterizing the SOA at these points, curves could be computed for a "high power" case (when the input currents are lower) and a "low power" case (when the input currents are higher), at the same frequency. Each of these curves are shown in Figure (5.2) and Figure (5.3) for the L-band and C-band lasers respectfully.

\section{L-Band SOA Characterization Curves}

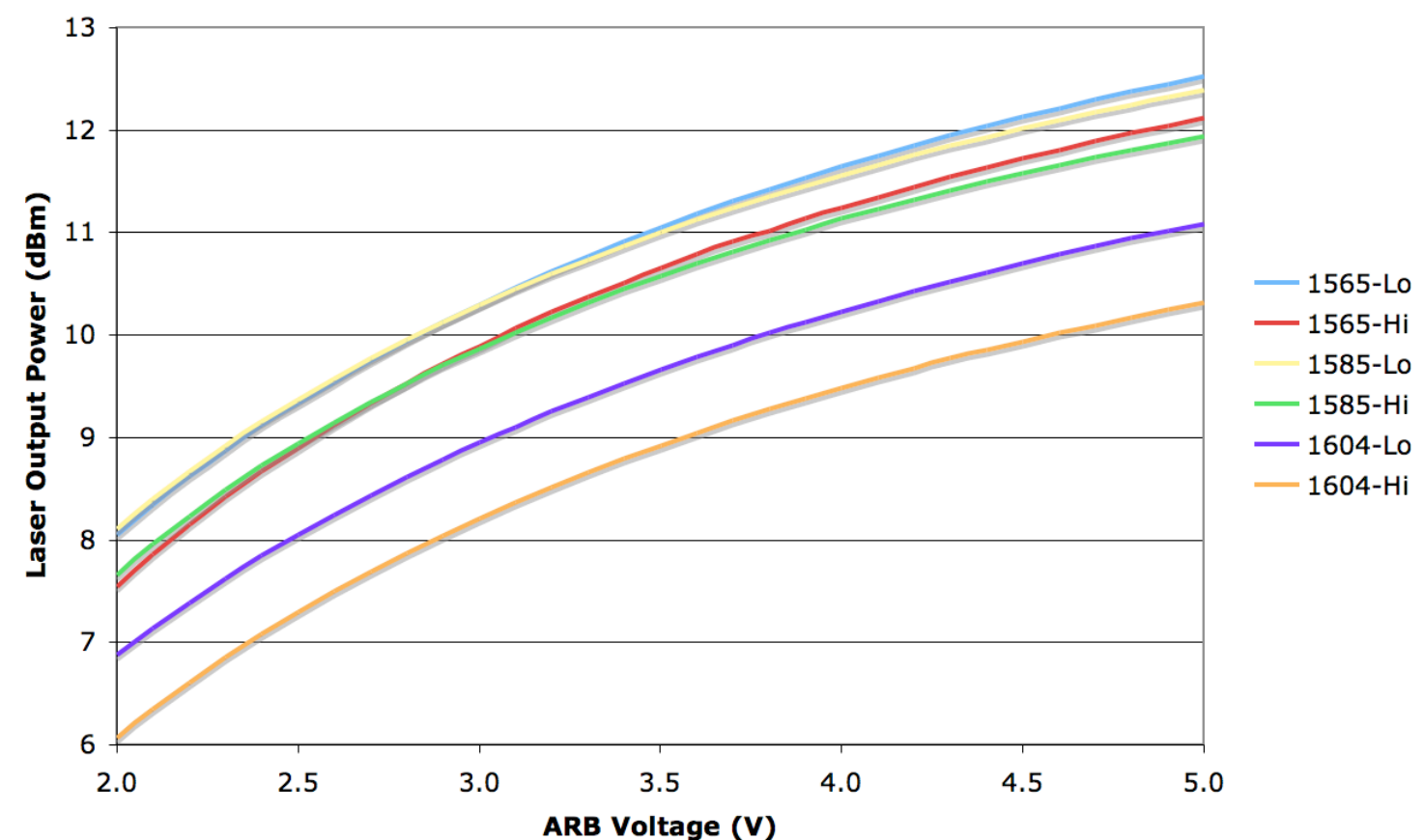

Figure 5.2: SOA Characterization Curves for L-band laser. Legend refers to the wavelength and whether the curve is low or high power characterization. The range of ARB voltages from $2 \mathrm{~V}$ to $5 \mathrm{~V}$ correspond to approximately $66 \mathrm{~mA}$ to $166 \mathrm{~mA}$. The SOA section is nominally biased at $4.5 \mathrm{~V}$, which corresponds to $150 \mathrm{~mA}$. 
C-Band SOA Characterization Curves

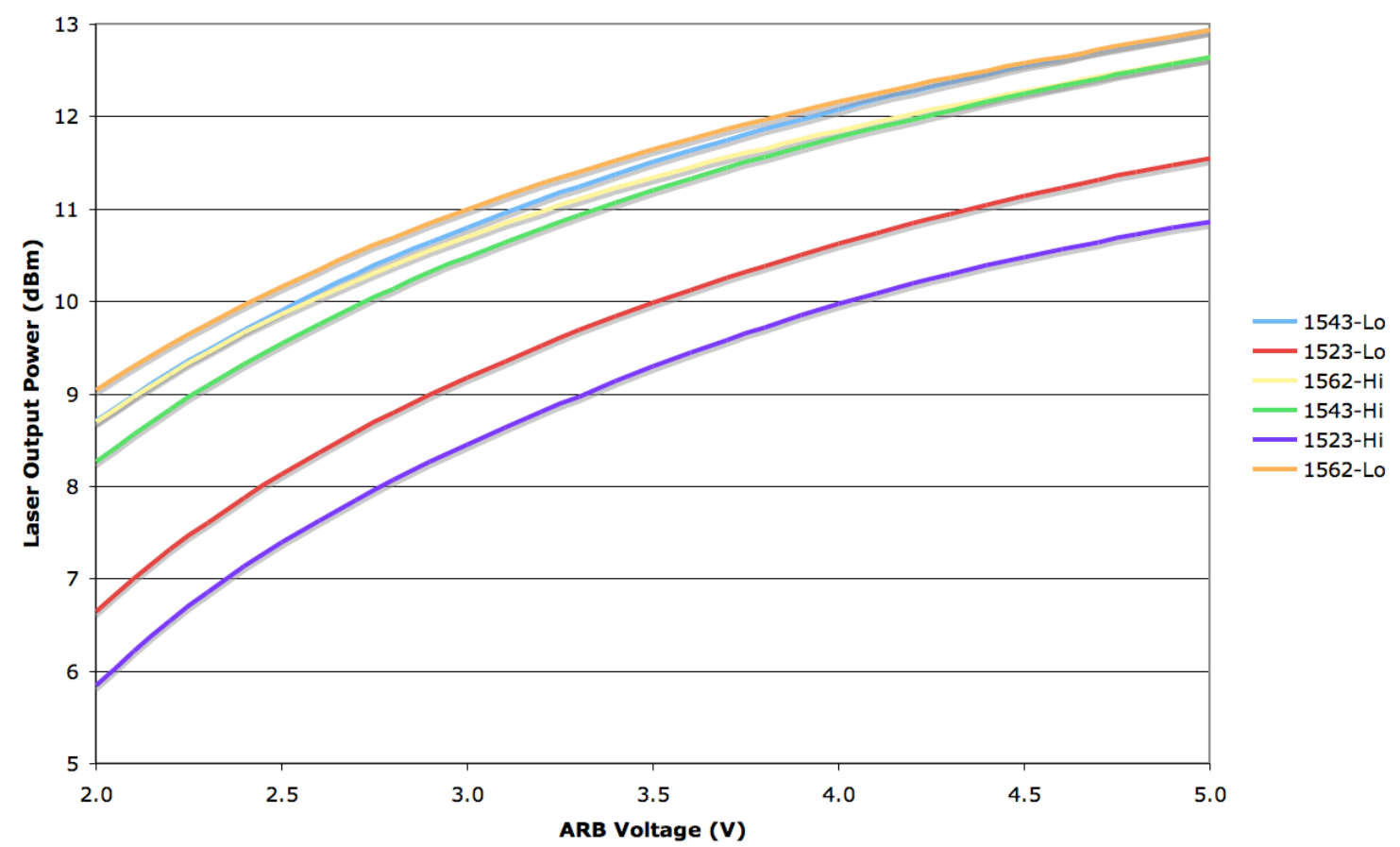

Figure 5.3: SOA Characterization Curves for C-band laser. Legend refers to the wavelength and whether the curve is low or high power characterization. The range of ARB voltages from $2 \mathrm{~V}$ to $5 \mathrm{~V}$ correspond to approximately $66 \mathrm{~mA}$ to $166 \mathrm{~mA}$. The SOA section is nominally biased at $4.5 \mathrm{~V}$, which corresponds to $150 \mathrm{~mA}$.

Without power leveling, the SOA section is D.C. biased with $4.5 \mathrm{~V}$ from an ARB (which corresponded with $150 \mathrm{~mA}$ of current). The power leveling will be implemented by varying the voltage supplied from this $\mathrm{ARB}$ by an appropriate amount. Since $4.5 \mathrm{~V}$ is the nominal amount, all the curves (shown in Figure (5.2) and Figure (5.3)) are shifted down by the value of the respective curve at 4.5 V. Figure (5.4) and Figure (5.5) show the shifted versions of the characterization curves for the L-band and C-band lasers respectively. Both Figure (5.4) and Figure (5.5) are used by giving a voltage needed to achieve a particular output power shift. These figures also show that the C-band laser's curves vary more than the L-band with respect to wavelength and power level. This will correspond to the C-band laser possessing a less ideal power leveled linear frequency ramp than the L-band laser's ramp. 


\section{L-Band Normalized SOA Characterization Curves}

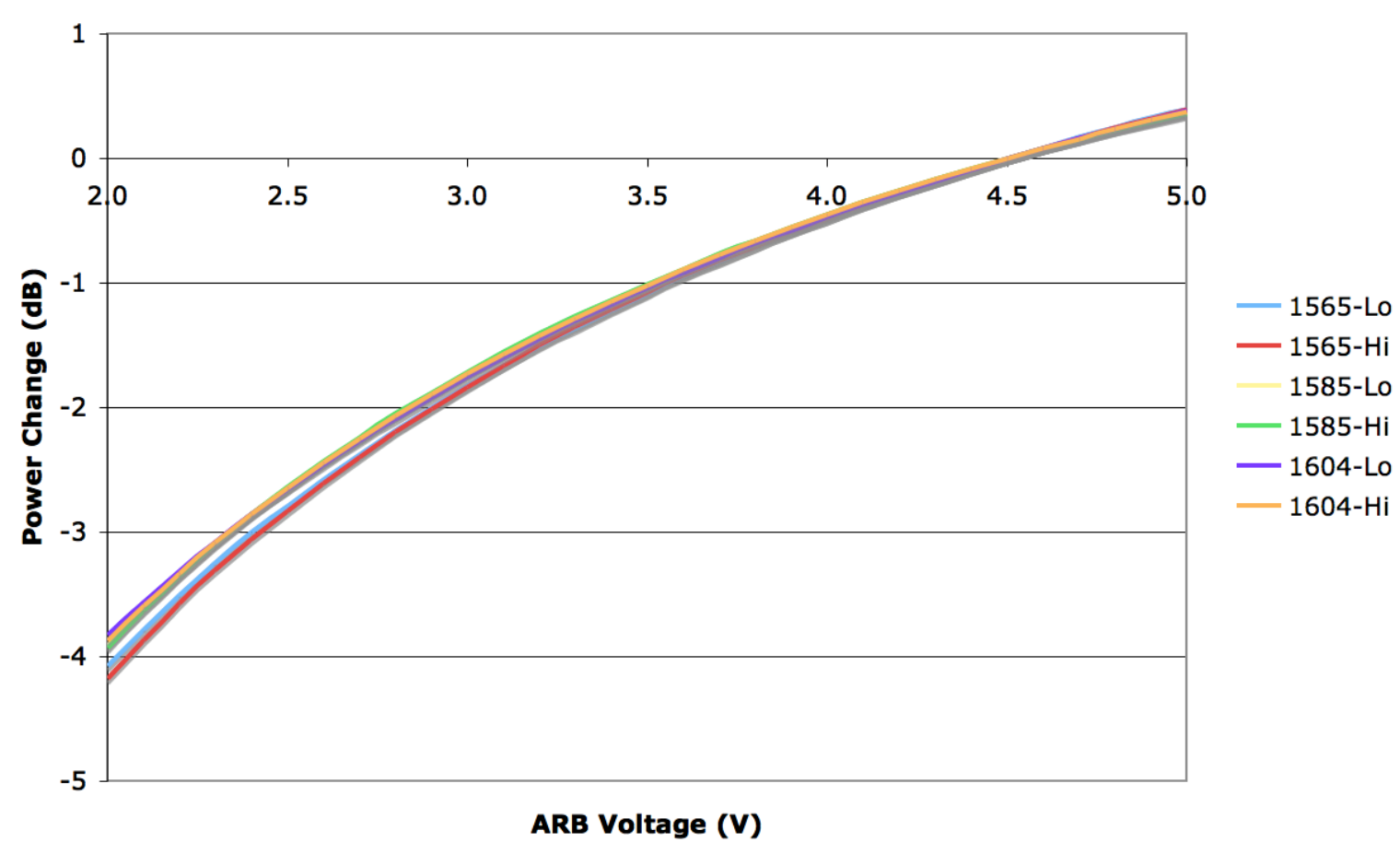

Figure 5.4: Shifted SOA Characterization Curves for L-band laser. Legend refers to the wavelength and whether the curve is low or high power characterization. The range of ARB voltages from $2 \mathrm{~V}$ to $5 \mathrm{~V}$ correspond to approximately $66 \mathrm{~mA}$ to $166 \mathrm{~mA}$. The SOA section is nominally biased at $4.5 \mathrm{~V}$, which corresponds to $150 \mathrm{~mA}$. Starting with Figure (5.2), each curve was shifted down by its value at $4.5 \mathrm{~V}$, which gives a set of curves when relate the change in output power to ARB voltage. 


\section{C-Band Normalized SOA Characterization Curves}

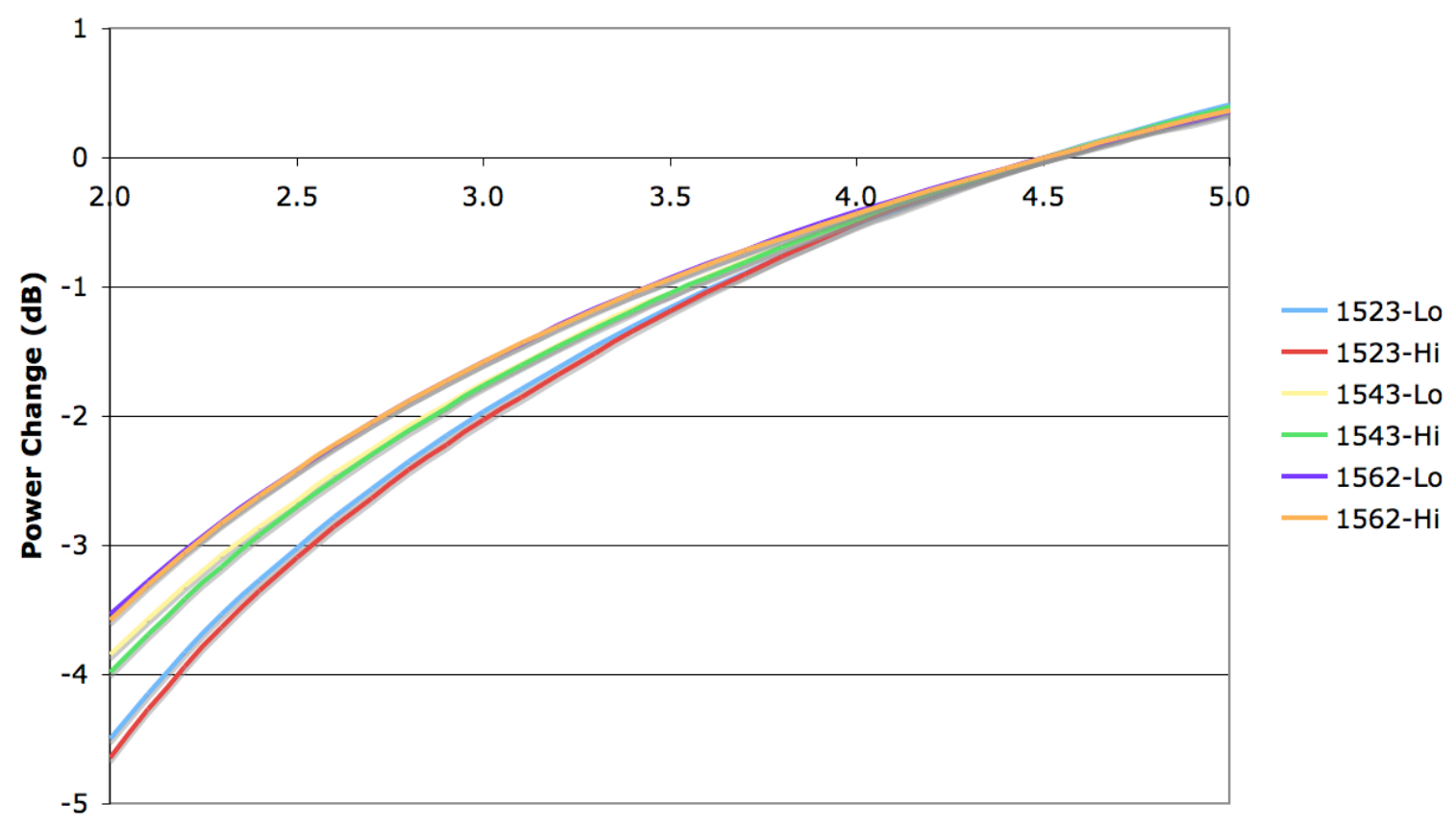

ARB Voltage (V)

Figure 5.5: Shifted SOA Characterization Curves for C-band laser. Legend refers to the wavelength and whether the curve is low or high power characterization. The range of ARB voltages from $2 \mathrm{~V}$ to

$5 \mathrm{~V}$ correspond to approximately $66 \mathrm{~mA}$ to $166 \mathrm{~mA}$. The SOA section is nominally biased at $4.5 \mathrm{~V}$, which corresponds to $150 \mathrm{~mA}$. Starting with Figure (5.3), each curve was shifted down by its value at 4.5 $\mathrm{V}$, which gives a set of curves when relate the change in output power to ARB voltage.

Next, the two sets of normalized SOA characterization curves for each wavelength are averaged together since they do not vary much. Each of the normalized SOA characterization curves contains thirty-one points and second order equations need to be fitted at each of these points that describe the change in power versus wavelength. Some of these curves for the L-band laser are shown in Figure (5.6). 
SOA Wavelength Dependence

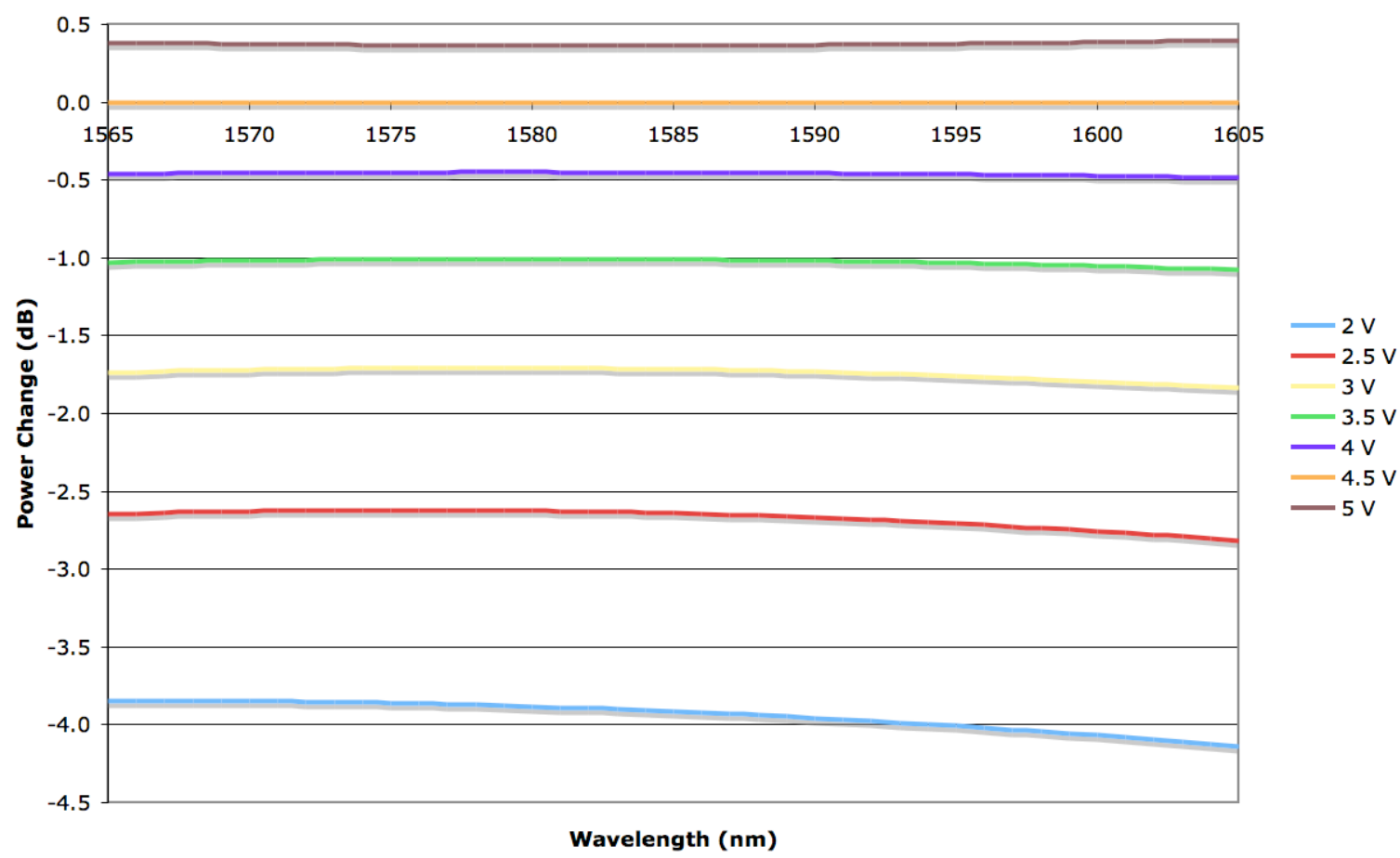

Figure 5.6: Curves demonstrate the wavelength dependence of the SOA laser output for various points along normalized SOA characterization curves. The $4.5 \mathrm{~V}$ curve, which is the reference level at $0 \mathrm{~dB}$, is the nominal power of the SOA. The range of ARB voltages from $2 \mathrm{~V}$ to $5 \mathrm{~V}$ correspond to approximately $66 \mathrm{~mA}$ to $166 \mathrm{~mA}$.

\section{2 - Reducing the Amplitude Ripple of the Frequency Ramp}

Changing the output power of the SG-DBR laser can be achieved relatively easily by modulating the current into the Semiconductor Optical Amplifier (SOA) section using another ARB. A new test setup, shown in Figure (5.7) is needed since four ARBs will be used. 


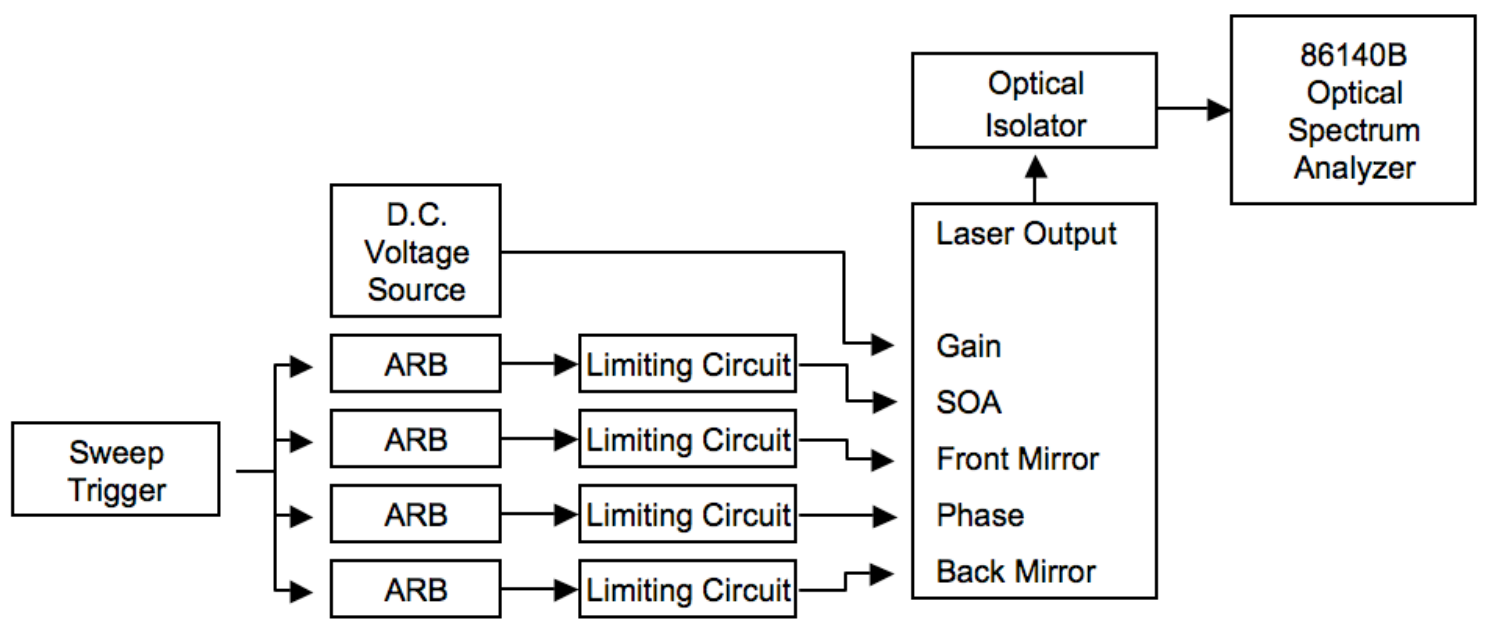

Figure 5.7: Test setup used for a power leveled linear frequency ramp. Another ARB is added to control the SOA voltage.

The leveled L-band and $\mathrm{C}$ band laser output levels were chosen to be $10.6 \mathrm{dBm}$ and $11.625 \mathrm{dBm}$ respectively. When the power level, shown in Figure (5.1), is higher than the power level chosen above; the SOA lowers the output power by decreasing the ARB voltage. When the power level is lower than the power level chosen above, the SOA raises the output power by increasing the ARB voltage. The SOA characterization curves shown in Figure (5.4) and Figure (5.5) are used to find a calculated SOA characterization curve that describes the ARB voltage versus laser output power for a particular wavelength. After this SOA characterization curve is calculated for a particular wavelength, the next step is to find the ARB voltage needed to level the output power. The difference between the power the laser should be leveled to and the power at the current point to be leveled is used in the particular characterization curve to find the ARB voltage that will successfully level the output. A text file that will control the output power level is created from the array of leveling ARB voltages found.

Figure (5.8) shows the output linear frequency ramp waveform from each laser running at slow speeds from the Agilent 86140B Optical Spectrum Analyzer as before in 
Figure (4.19). Note the drastic improvement in the output power ripple compared to

Figure (4.19).

Power Leveled Linear Frequency Ramps

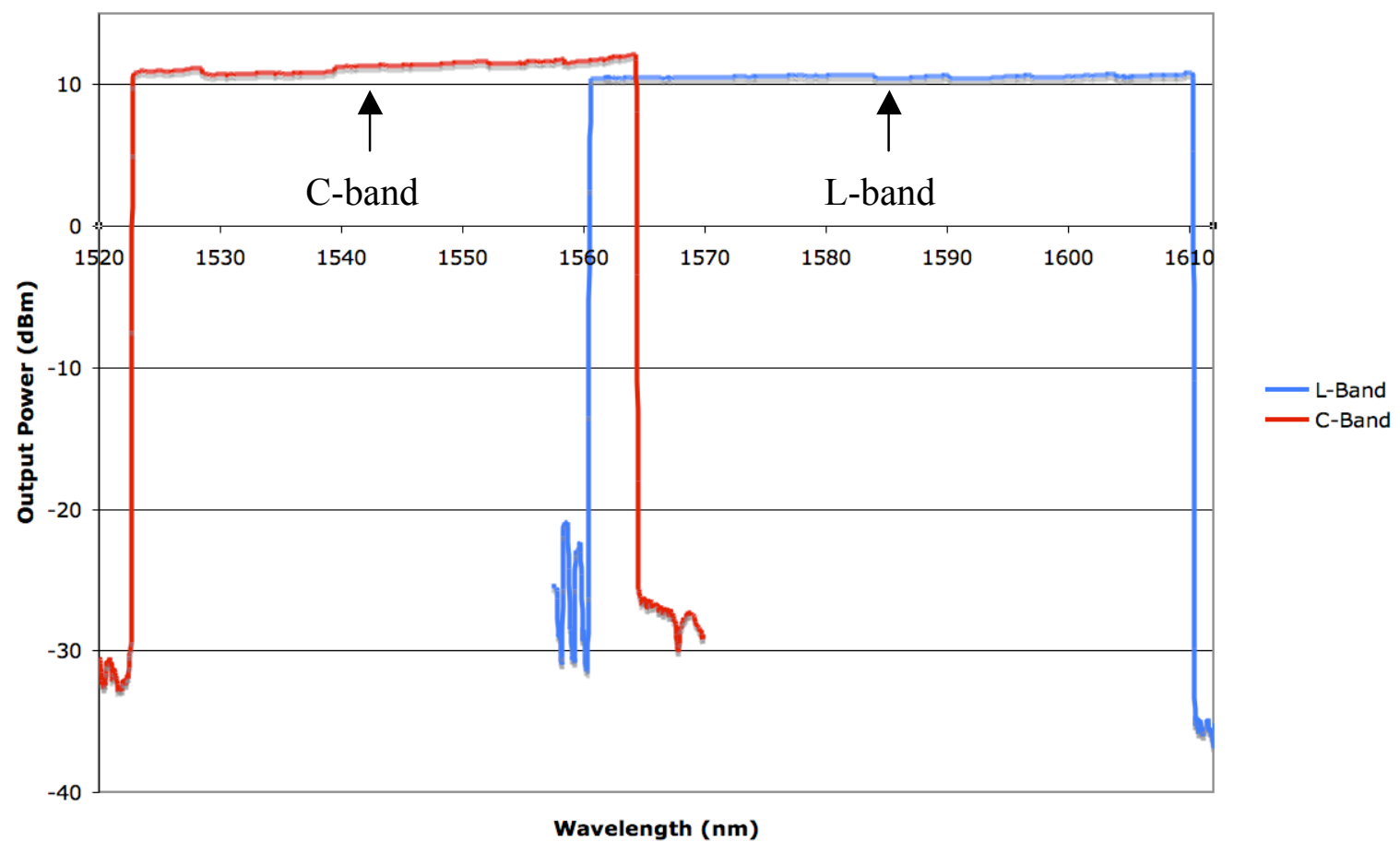

Figure 5.8: Power leveled frequency ramps of $\mathrm{L}$-band and $\mathrm{C}$-band lasers run at a $1 \mathrm{~Hz}$ repetition rate. Note the overlap of the $\mathbf{L}$-band and $\mathrm{C}$-band frequency ramps, which allow for concatenation of the two ramps into a single ramp. Also note the reduced amplitude ripple compared to Figure (4.19).

Table (5.1) compares statistical data for the original frequency ramp shown in Figure (4.19) and the power leveled frequency ramp shown in Figure (5.8). The table shows average and standard deviation values for the output power of the lasers.

\begin{tabular}{|c|c|c|c|c|}
\hline \multicolumn{5}{|c|}{ Statistics for the Reduction of Amplitude Ripple During Frequency Ramp } \\
\hline Type & Original L-band & Original C-band & P.L. L-band & P.L. C-band \\
\hline Average & $12.200 \mathrm{dBm}$ & $12.952 \mathrm{dBm}$ & $10.563 \mathrm{dBm}$ & $11.282 \mathrm{dBm}$ \\
\hline Std. Deviation & $0.5526 \mathrm{dBm}$ & $0.4821 \mathrm{dBm}$ & $\mathbf{0 . 0 8 7 5} \mathrm{dBm}$ & $0.3747 \mathrm{dBm}$ \\
\hline
\end{tabular}

Table 5.1: Table gives values for the average values and standard deviation of the output power for the original L-band, original C-band, power leveled L-band, and power leveled C-band frequency ramps.

Power leveling, as described in this chapter, drastically reduces the amplitude ripple in the L-band laser. The ripple in the power leveled version is more than six times less than 
in the original frequency ramp. Standard deviation of the C-band power leveled frequency ramp's amplitude ripple, however, only decreases by $22.3 \%$. Even though the L-band laser's amplitude ripple was originally worse than the C-band, the L-band laser's SOA characterization curves (shown in Figure (5.4)) are closer together than the C-band laser's (shown in Figure (5.5)). This results in less reduction of the amplitude ripple. However, one should note that the high frequency ripple of the C-band frequency ramp is successfully reduced.

The frequency ramps shown in Figure (5.8) are a result of a single pass of leveling. Further passes of leveling the power would result in greatly increased amplitude ripple attenuation, especially the C-band. A more advanced test structure would abandon the open loop structure shown in Figure (5.7) for a closed loop system that would monitor the power level and adjust the SOA accordingly to reduce the amplitude ripple even more. A closed loop test structure would be needed to completely remove the L-band and C-band laser's amplitude ripple. 


\section{Chapter 6 - Concatenation of L-band and C-band Lasers to Get a Continuous Frequency Ramp}

50 continuous tuning segments are stitched together to create the L-band and Cband linear frequency ramps. In a similar way, the two linear frequency ramps can be concatenated to create a single linear frequency ramp with approximately twice the bandwidth. This procedure could also be expanded by concatenating $\mathrm{N}$ number of linear frequency ramps, with overlapping frequency coverage, together to create a single linear frequency ramp with approximately $\mathrm{N}$ times the original bandwidth. This is important since time resolution in an OCT measurement is directly proportional to the spectral width of the source. The two SG-DBR lasers are concatenated by attaching each laser output to a $-3 \mathrm{~dB}$ coupler as shown in Figure (6.1).

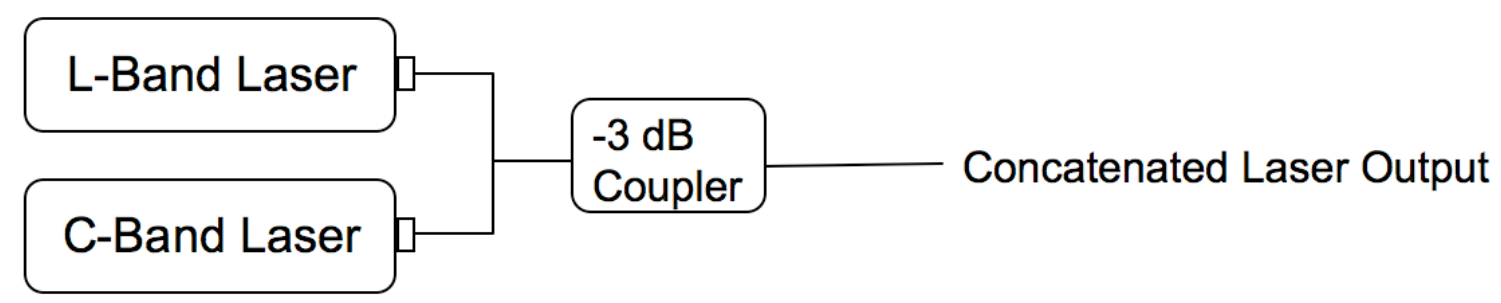

Figure 6.1: Concatenation of L-band and C-band lasers using a -3 dB coupler.

Using this procedure, a method is needed for shuttering the output of the laser not currently in operation. The SOA section of the laser can provide approximately $50 \mathrm{~dB}$ of attenuation when the input voltage of the ARB is set to zero. With the input voltage of the ARB set to zero the input becomes a short, which turns the semiconductor optical amplifier into an optical attenuator. Although the SOA can provide $50 \mathrm{~dB}$ of attenuation, some wavelengths are attenuated less. Figure (6.2) shows a test setup created to scan over the bandwidth of the laser with the SOA shorted to measure the attenuation versus wavelength. Shorting the SOA results in maximum attenuation. 


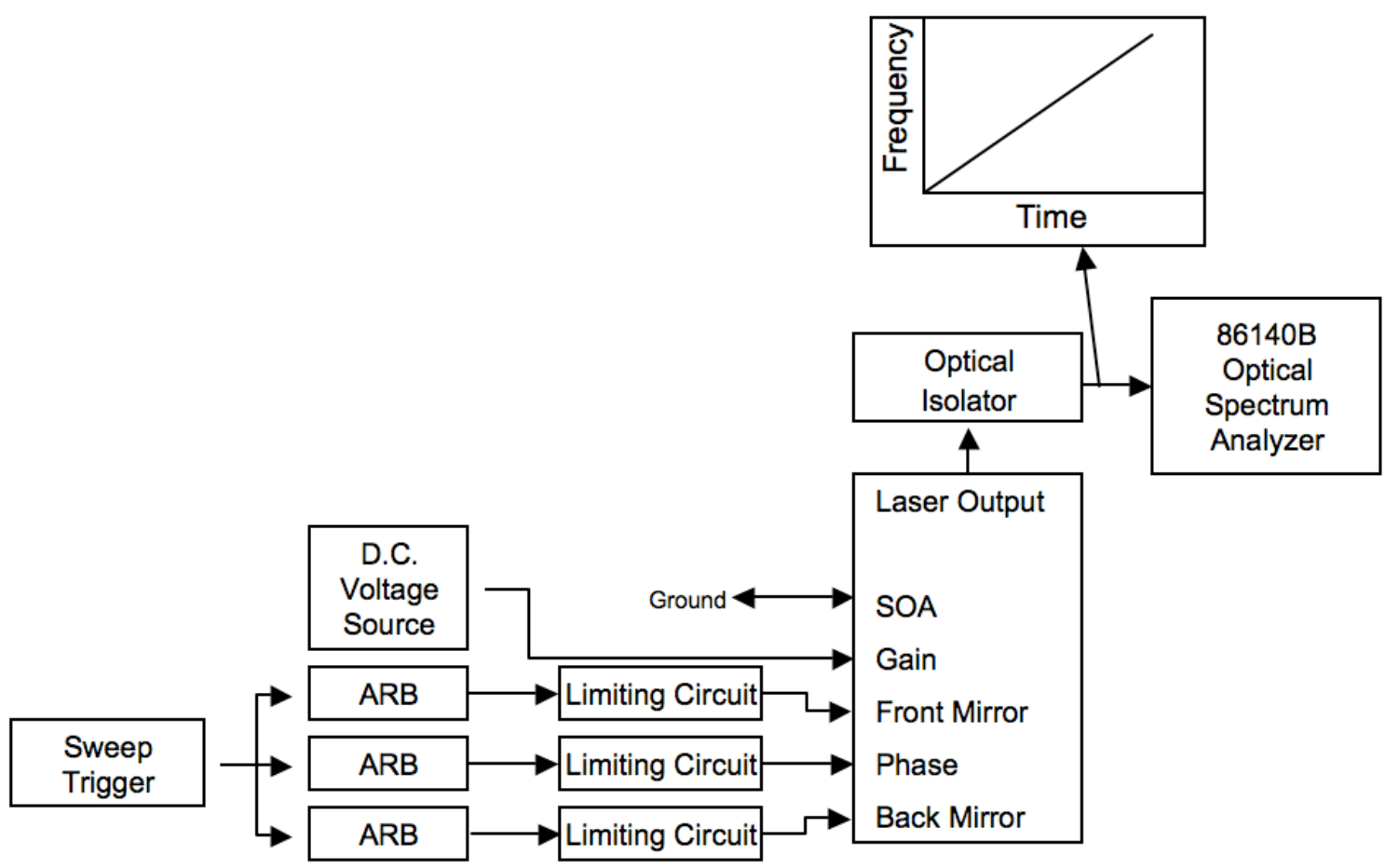

Figure 6.2: Test setup used to find the attenuation of the SOA versus wavelength shown in Figure (6.3) and Figure (6.4). The three ARBs control the front mirror, back mirror, and phase sections to create the linear frequency ramp. The SOA is shorted, which occurs when the SOA is set to $0 \mathrm{~V}$, which attenuates the laser output. The gain is set to $100 \mathrm{~mA}$. The OSA records the attenuation of the laser as the frequency ramp runs.

Figure (6.3) and Figure (6.4) show the attenuation due to the SOA port shorted with respect to wavelength from the setup used in Figure (6.2). 
L-Band Laser Minimum SOA Attenuation

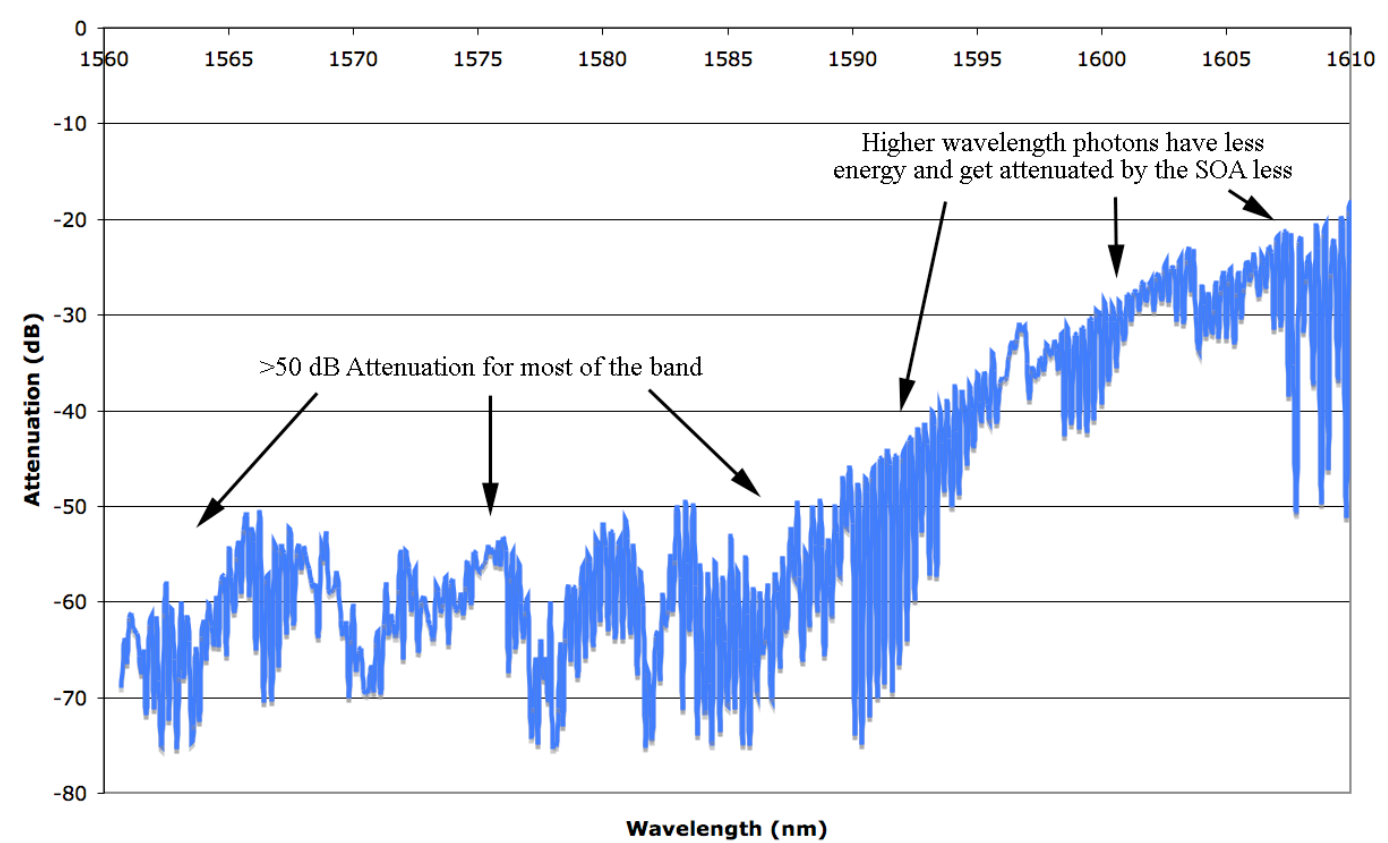

Figure 6.3: SOA attenuation versus wavelength measurement due to L-band ARB SOA input voltage set to zero volts. The higher wavelength (lower energy) photons get absorbed less since the bandgap of the SOA is larger than the energy of the incident photons.

\section{C-Band Laser Minimum SOA Attenuation}

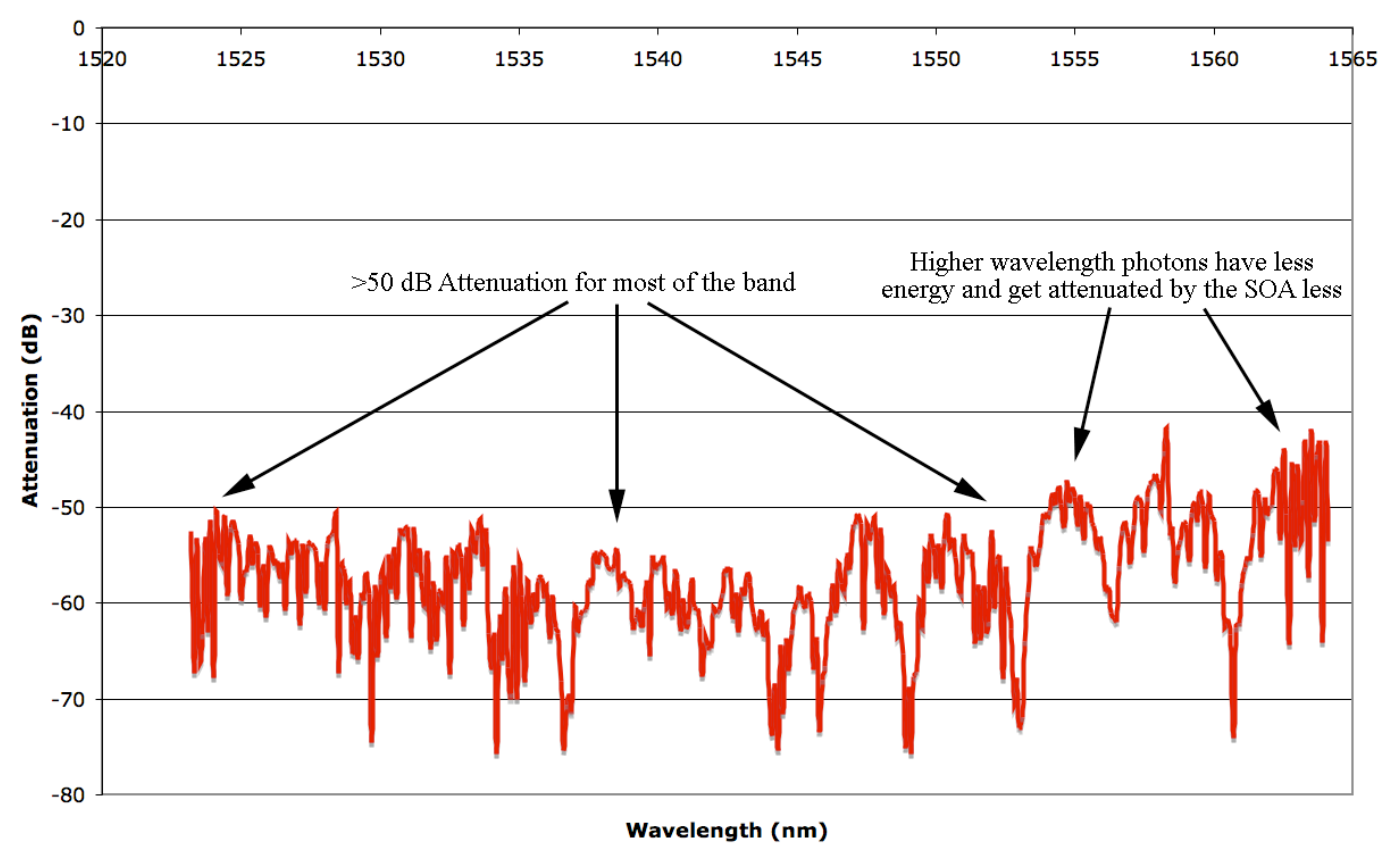

Figure 6.4: SOA attenuation versus wavelength measurement due to C-band ARB SOA input voltage set to zero volts. The higher wavelength (lower energy) photons get absorbed less since the bandgap of the SOA is larger than the energy of the incident photons. 
One can see that the higher wavelengths of the L-band laser do not get attenuated nearly as much as the L-band lasers lower wavelengths. A similar, but smaller, effect occurs on the C-band laser. This is due to the inability of the built-in bandgap of the SOA to absorb the lower energy photons of the higher wavelength output. To combat this, when the SOA is set to 'off' the laser's output wavelength will be set to the lowest value, $1564 \mathrm{~nm}$ and $1523 \mathrm{~nm}$ for the L-band and C-band respectively during the sweep. When a proper SOA attenuation wavelength is set, Figure (6.5) shows the block diagram test setup needed to analyze the laser attenuation of the laser output of the C-band laser with the SOA 'on' and 'off,' plot shown in Figure (6.6). Note the SOA cannot attenuate the higher wavelength amplified spontaneous emission (ASE) noise as well as the lower wavelength ASE noise. Once again this is due to the bandgap of the SOA being too large to stop the lower energy photons.

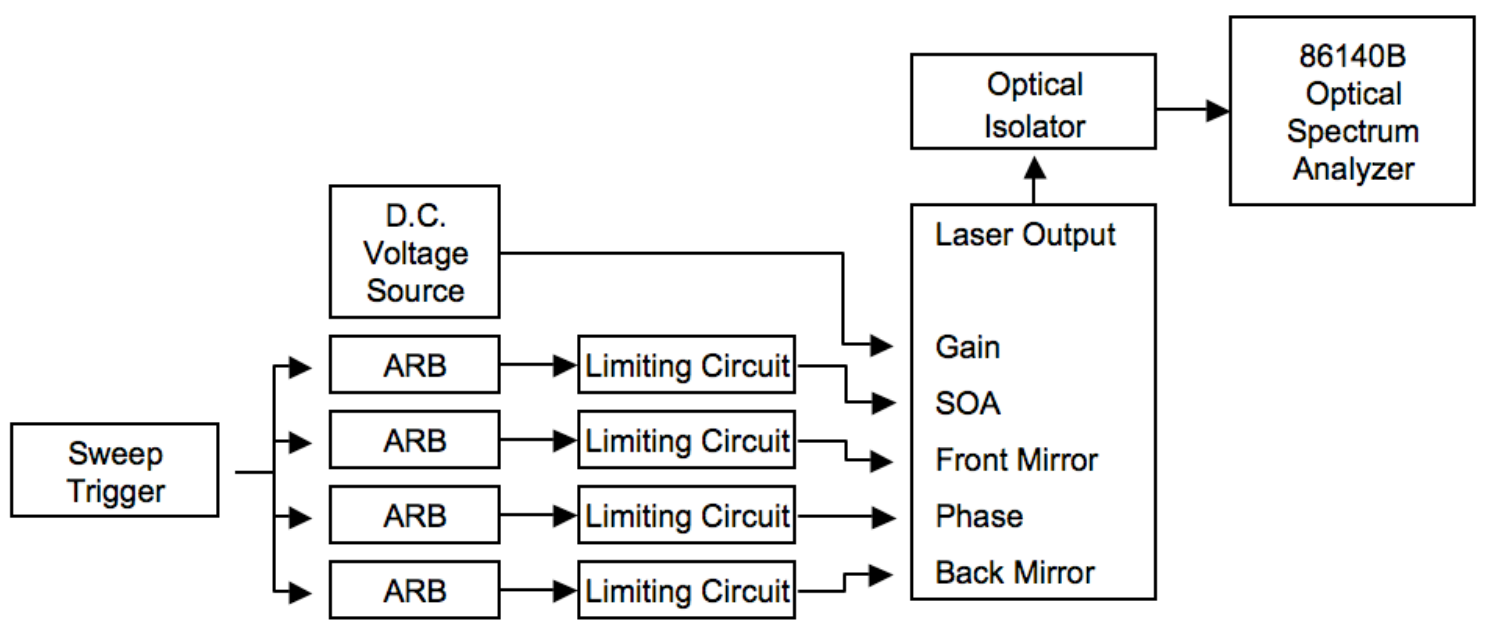

Figure 6.5: Test setup used to analyze the laser output, in Figure (6.6) when the ARB SOA is biased at $4.5 \mathrm{~V}$ and set to $0 \mathrm{~V}$ which shorts the input. Setup also used in the test shown in Figure (6.7) which analyzes the switching times of the SOA section. 
SOA Laser Output Attenuation

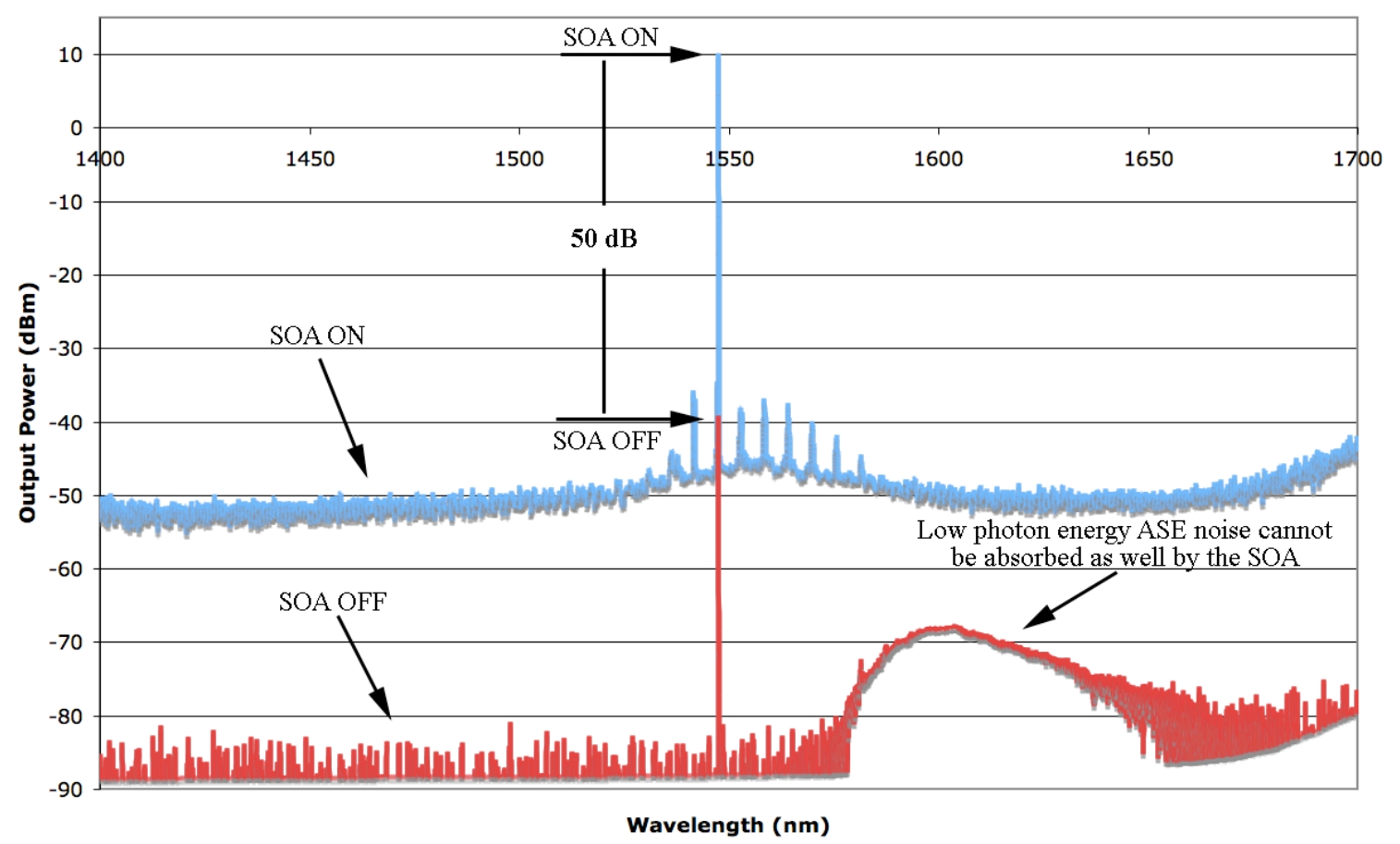

Figure 6.6: Figure shows the attenuation of the laser output due to turning off the SOA section. When the SOA is "on" the laser provides $>10 \mathrm{dBm}$ of peak output power. When the SOA is "off," which shorts the input, the SOA becomes an optical attenuator with approximately $50 \mathrm{~dB}$ of attenuation. The SOA cannot stop the lower energy photons, however, which causes low energy ASE noise to feed through at higher wavelengths.

With the optical shutter chosen, the rise and fall times of the SOA section must be determined. Since the concatenated laser ramp will begin with the L-band laser and then switch to the C-band laser, the L-band SOA fall time and the C-band SOA rise time will be calculated. The L-band SOA fall time and the C-band SOA rise time will determine the dead time between the L-band and C-band laser frequency ramps. Also, the SOA performance depends on the rise and fall times of the Agilent Arbitrary Waveform Generator since the ARBs electrically control the SOA section.

The test setup in Figure (5.7) is used to calculate the rise and fall times of the SOA section. A square wave with a high value of $4.5 \mathrm{~V}$, and low value of $0 \mathrm{~V}$, a $10 \mathrm{kHz}$ repetition rate, and a $50 \%$ duty cycle drives the SOA. Figure (6.6) shows the L-band 
SOA rise time, the C-band SOA fall time, and an example of the ARB step function.

From this data the rise and fall times are calculated to be approximately $50 \mathrm{~ns}$, however, it is also shown that these times are primarily caused by the rise and fall waveforms of the ARB.

Laser SOA Rise and Fall Waveforms

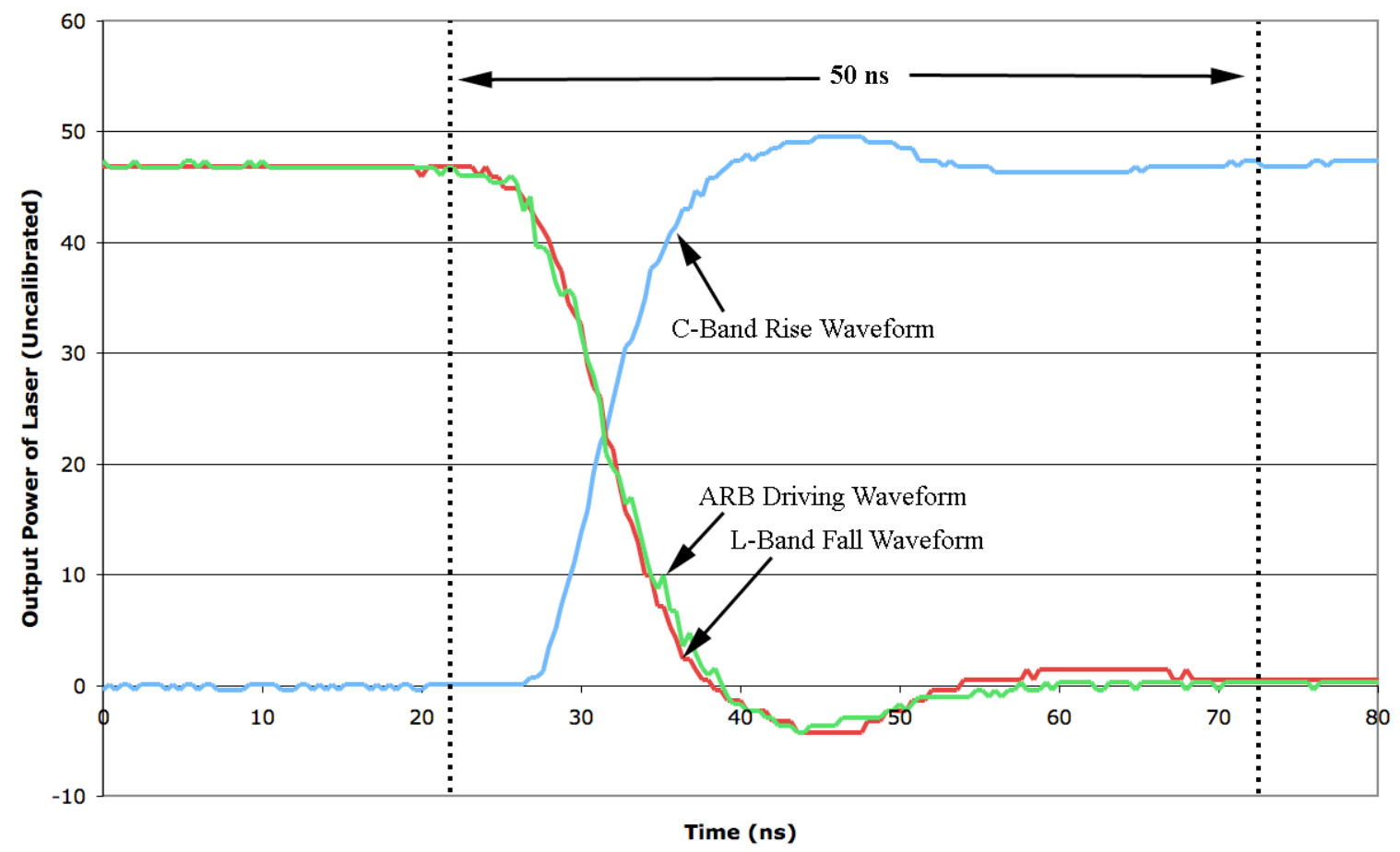

Figure 6.7: Shows C-band SOA rise waveform, L-band SOA fall waveform, and the ARB driving waveform for the L-band SOA section. Test setup in Figure (6.5) is used to for the test shown in Figure (6.7). There is a 50 ns dead time while the ARB either turns the SOA on or off.

The $50 \mathrm{~ns}$ needed to switch from either on to off or off to on will correspond to a $50 \mathrm{~ns}$ time when the laser output is undetermined. The errors caused by the concatenation point are comparable to a single stitching error needed to append two continuous tuning segments.

Switching between the L-band and C-band lasers while both are biased at a single wavelength will isolate the effects of the concatenation point on the lasers. A new test 
setup, shown in Figure (6.8), is needed to switch between two single frequency biased SG-DBR lasers.

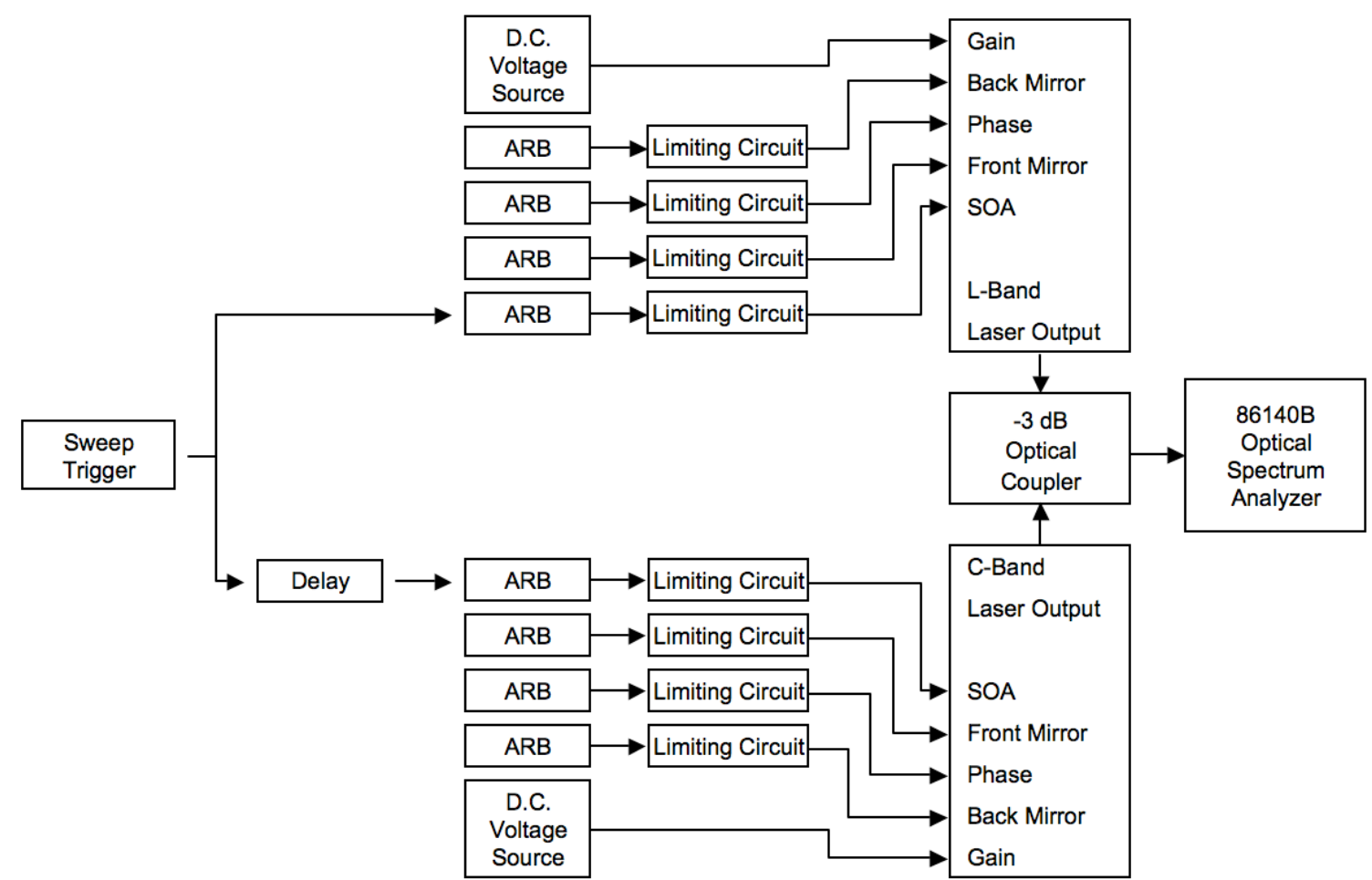

Figure 6.8: Test setup used for switching between the two lasers at a single frequency.

A cross-over frequency that is contained in both the L-band and C-band tuning dictionaries must be chosen first. The phase, front mirror, and back mirror ARBs for each laser must be set to voltages that correspond to the chosen frequency. Table (6.1) contains biasing information for the two lasers.

\begin{tabular}{|c|c|c|}
\hline & L-Band & C-Band \\
\hline Switching Wavelength & $1568.500 \mathrm{~nm}$ & $1568.500 \mathrm{~nm}$ \\
\hline Phase Voltage & $3.4221709 \mathrm{~V}$ & $1.4450000 \mathrm{~V}$ \\
\hline Front Mirror Voltage & $0.9441109 \mathrm{~V}$ & $1.6376000 \mathrm{~V}$ \\
\hline Back Mirror Voltage & $2.3064531 \mathrm{~V}$ & $\mathbf{0 . 6 6 2 7 4 0 0} \mathrm{V}$ \\
\hline
\end{tabular}

Table 6.1: Bias points for single frequency $\mathrm{L}$-band and C-band switching test.

Since there are two separate paths of the sweep trigger signal, they reach the Lband and the C-band ARBs at different times. The delay of the signal reaching the C- 
band ARB after it reached the L-band ARB is $\Delta \mathrm{t}=160.4 \mathrm{~ns}$ for the particular test setup shown in Figure (6.8). This delay can be calibrated out since the C-band trigger signal path incorporates a variable delay. The time that each laser is on or off is set to $10 \mu \mathrm{s}$ since this corresponds to the maximum repetition rate of the linear frequency ramp. This corresponds to a sweep trigger function generator frequency setting of $f=50 \mathrm{kHz}$. Figure (6.9) shows the results of the single frequency switching test. The variable delay was fine tuned to a value of $\mathrm{t}_{\text {delay }}=2.45 \mu \mathrm{s}$ to optimize the stitching point, meaning the L-band fall and C-band rise waveforms overlap as much as possible (shown in Figure (6.7)).

Figure (6.9) effectively shows the approximately $50 \mathrm{~ns}$ of dead time during the laser switching. At the switching point, both lasers are slightly changing frequencies quickly, which causes rapid fluctuations between constructive and destructive interference.

Single Frequency Laser Test (Small Scale)

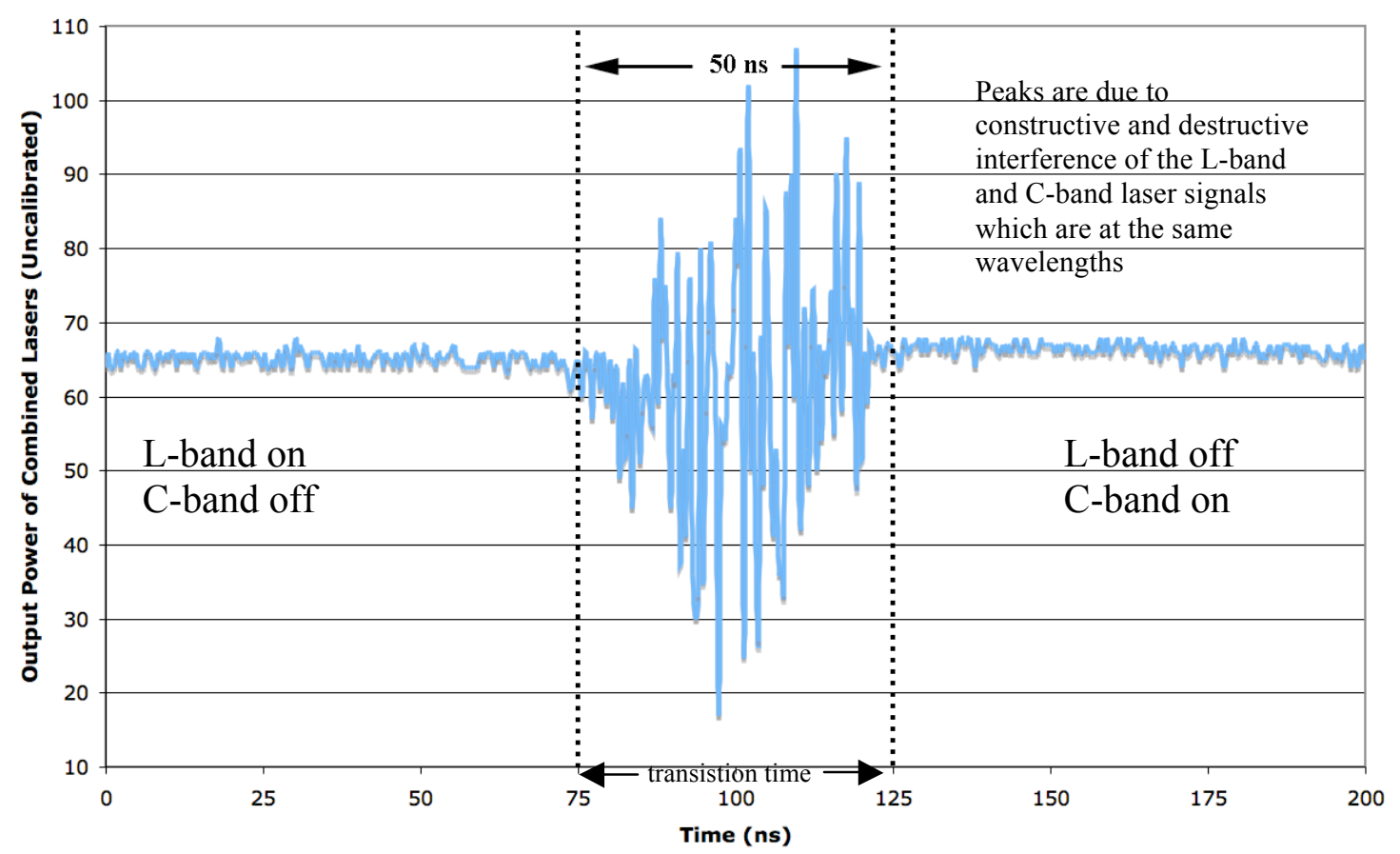

Figure 6.9: Zoomed in plot of switching point between L-band and C-band lasers, each biased at $1568.500 \mathrm{~nm}$ and outputs combined. During the $50 \mathrm{~ns}$ dead time the two laser outputs changes between constructive and destructive interference quickly. Lasers both run at a $50 \mathrm{kHz}$ repetition rate. 
A revised version of Figure (6.8) is needed to switch between lasers as well as allow for linear frequency ramps. Putting these two requirements together will give a test setup, shown in Figure (6.10), for concatenating two power leveled linear frequency ramps.

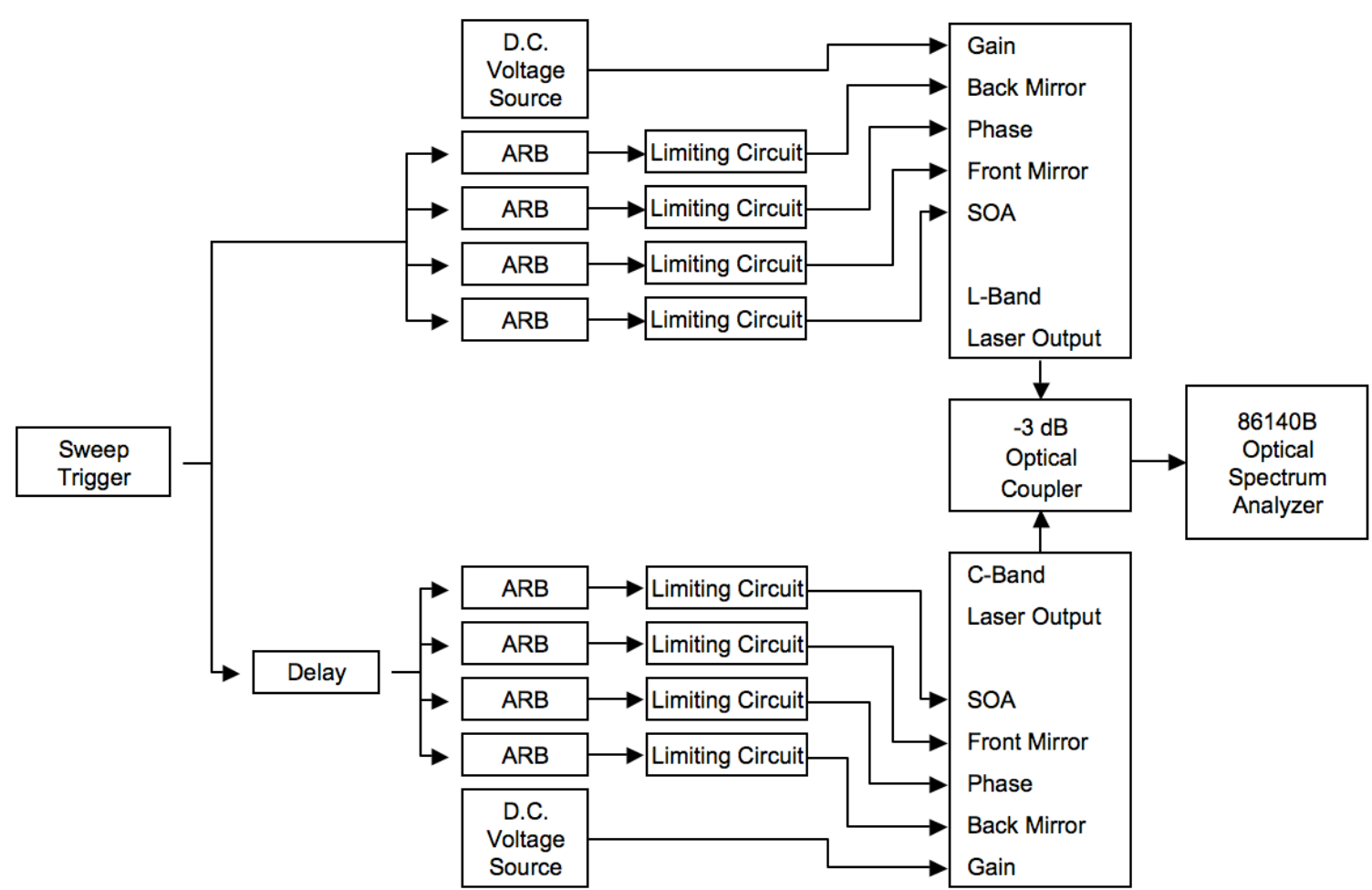

Figure 6.10: Test setup for the concatenation of two power leveled linear frequency ramps into a single power leveled linear frequency ramp.

Simple modifications must be performed to the L-band and C-band tuning dictionaries to concatenate two power leveled linear frequency ramps into a single continuous sweep. First, a frequency must be chosen to switch between the L-band and C-band frequency ramps. $1564 \mathrm{~nm}$ was chosen to be the switching wavelength, which corresponds to a switching frequency of $191.816 \mathrm{THz}$. Second, the L-band tuning dictionary must be modified so the ramp ends at $1564 \mathrm{~nm}$, while the C-band tuning dictionary must be cropped so the ramp starts at $1564 \mathrm{~nm}$. From this one gets the tuning 
waveforms needed, shown in Figure (6.11), for concatenating two power leveled linear frequency ramps together. The L-band laser waveforms begin with the power leveled linear frequency ramp. After the L-band laser ramp is finished, the SOA is turned off and the phase, front mirror, and back mirror are set to a frequency that gives good SOA attenuation. Conversely the C-band laser waveforms begin with the SOA turned off and the phase, front mirror, and back mirror set to a frequency that also gives good SOA attenuation. When the L-band laser ramp is completed the C-band laser ramp waveforms simultaneously continue the power leveled linear frequency ramp. 

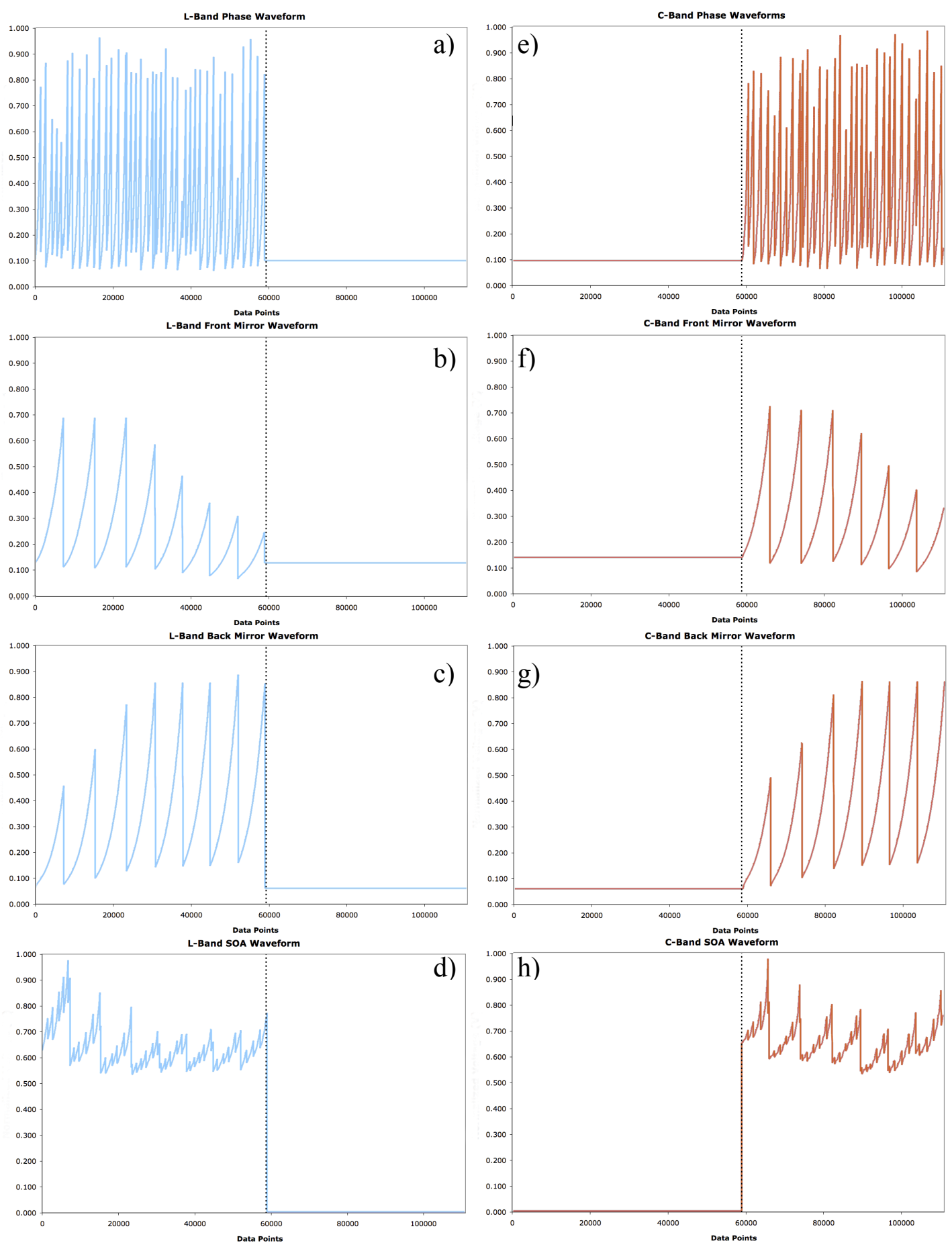

Figure 6.11: ARB tuning waveforms needed for creating a single power leveled linear frequency ramp with approximately twice the bandwidth from two separate SG-DBR laser sweeps. The dotted

lines represent the switching point between the $\mathrm{L}$-band and $\mathrm{C}$-band laser ramps. $\mathrm{X}$-axis is represented by data points of the waveforms and the $\mathrm{Y}$-axis is normalized ARB voltage. Image "a" is the input waveform to the $L$-band phase section. Image " $b$ " is the input waveform to the $L$-band front mirror section. Image " $c$ " is the input waveform to the L-band back mirror section. Image "d" is the input waveform to the $\mathrm{L}$-band SOA section. Image " $\mathrm{e}$ " is the input waveform to the C-band phase section. Image " $\mathrm{f}$ " is the input waveform to the $\mathrm{C}$-band front mirror section. Image " $\mathrm{g}$ " is the input waveform to the $C$-band back mirror section. Image " $h$ " is the input waveform to the $C$-band SOA section. 
Loading these waveforms into the ARBs and running the concatenated power leveled frequency ramp gives the trace shown in Figure (6.12).

Concatenated L-Band and C-Band Power Leveled Linear Frequency Sweep

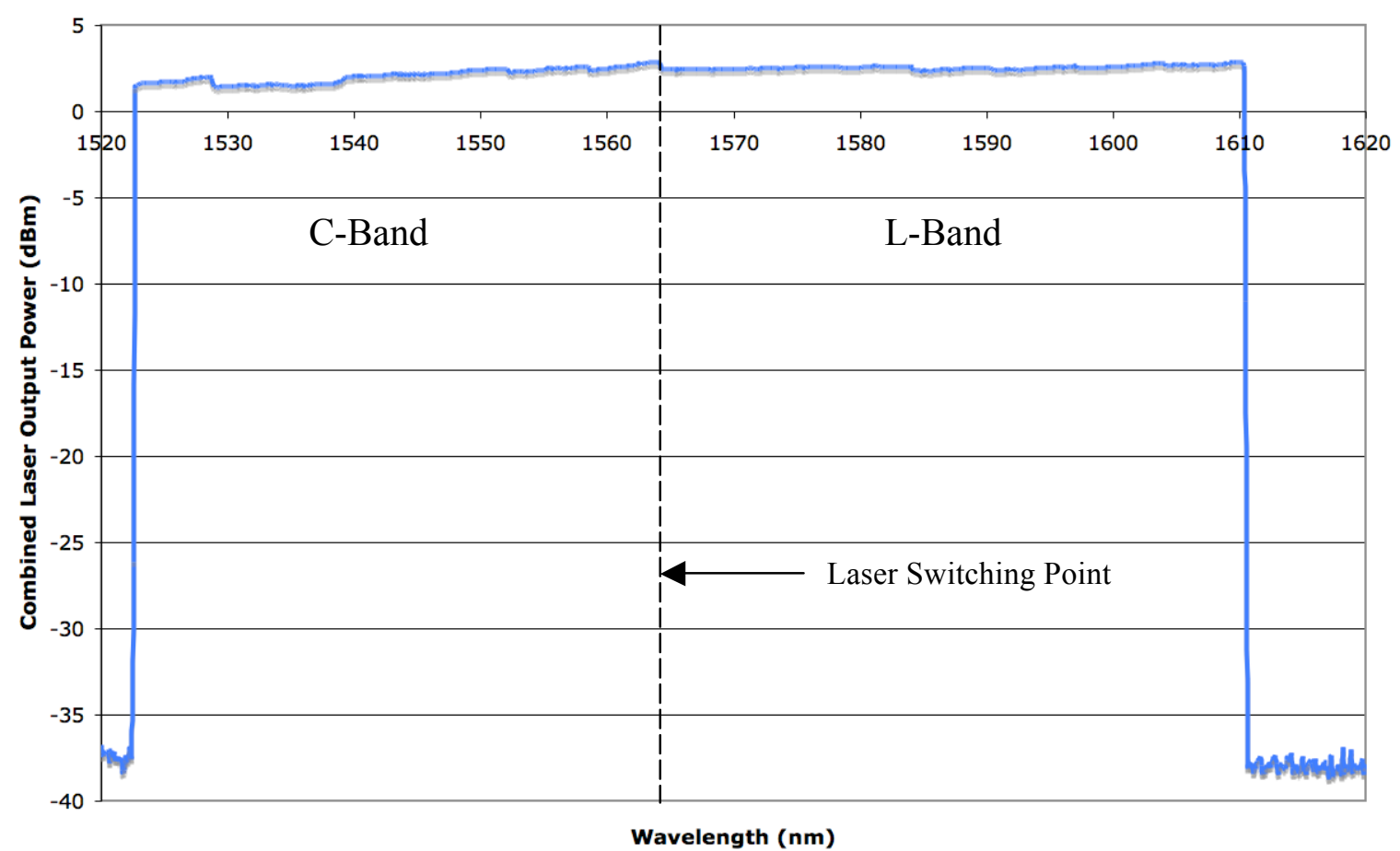

Figure 6.12: Concatenated power leveled linear frequency ramps from an L-band and C-band SGDBR laser at a $1 \mathrm{~Hz}$ repetition rate.

The single L-band and C-band linear frequency ramps have $49.7 \mathrm{~nm}$ and $41.2 \mathrm{~nm}$ of bandwidth respectively. Concatenating the L-band and C-band lasers together brings the bandwidth from $49.7 \mathrm{~nm}$ (for the L-band laser) to a total of $87.3 \mathrm{~nm}$ or $75.7 \%$ more. From Equation (3.8), OCT resolution should increase $43.1 \%$.

Since concatenating the L-band and C-band laser increased the bandwidth of the OCT source. Future research should be able to concatenate additional lasers with adjacent spectral coverage to increase the bandwidth further. Increasing the bandwidth of 
the source by adding additional lasers will be advantageous for OCT since increasing bandwidth results in increased time resolution. 


\section{Chapter 7 - Stitching Errors and OCT System Implications}

Chapter 4 and Chapter 5 outlined the procedure necessary to create a power leveled linear frequency ramp using a L-band or C-band SG-DBR laser. While the power leveled linear frequency ramps generated have great attributes, such as high repetition rates and minimum $10 \mathrm{dBm}$ output power, they are not perfect. There are two parameters of concern with stitching errors. First, is how well the wavelengths matchup before and after a stitch. Second, how long does the output wavelength of the laser stay undefined after a stitch.

Chapter 7 analyzes the linearity of the SG-DBR laser frequency ramps. Section 7.1 outlines the causes of stitching errors. Techniques for minimizing stitching errors at slow $(100 \mathrm{~Hz})$ and fast $(10 \mathrm{kHz})$ rates are detailed in Section 7.2. Physical limits on stitching errors are calculated in Section 7.3. Finally in Section 7.4, the implications for imperfect stitching errors are analyzed.

\section{1 - Sources of Stitching Errors}

As previously stated in Section 4.2, stitching errors primarily limit the linearity of the frequency ramps. Continuous tuning segments are limited to approximately $1 \mathrm{~nm}$ due to the current limitations of the phase section of the SG-DBR laser. So to cover the entire $50 \mathrm{~nm}$ bandwidth of the SG-DBR laser with a linear frequency ramp, approximately 50 continuous tuning segments must be joined together into a single ramp. Figure (7.1) shows how the 8 tuning paths are stitched together. 


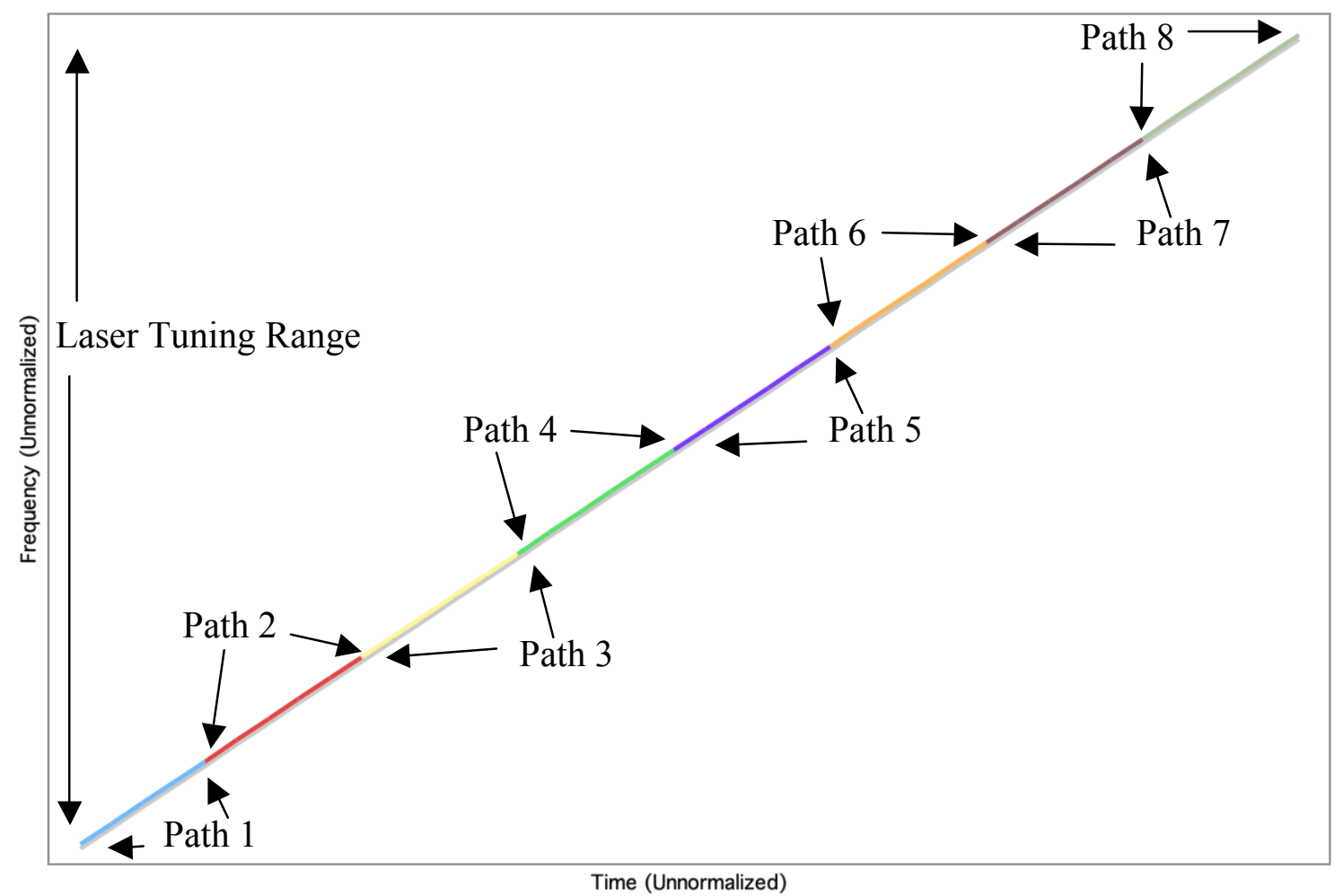

Figure 7.1: Image shows an example of the 8 tuning paths stitched together to create a single frequency ramp. Figure shows where each of the 8 paths start and end as well as the range of frequencies covered by the frequency ramp. Each path is itself made up of approximately 6 continuous tuning segments which are stitched together as above to create a path. Figure (7.2) shows how errors occur at a stitch.

The characterization of each continuous tuning segment was performed separately and care is taken to assure the continuous tuning segments do not overlap or omit frequencies. However, when running the frequency ramp, transients not present during characterization can cause a "stitching" error. A stitching error occurs when stitching two continuous tuning segments are joined and the laser frequency suddenly jumps forward or backwards at the stitching point. Figure (7.2) gives examples of these two types of stitching errors. 

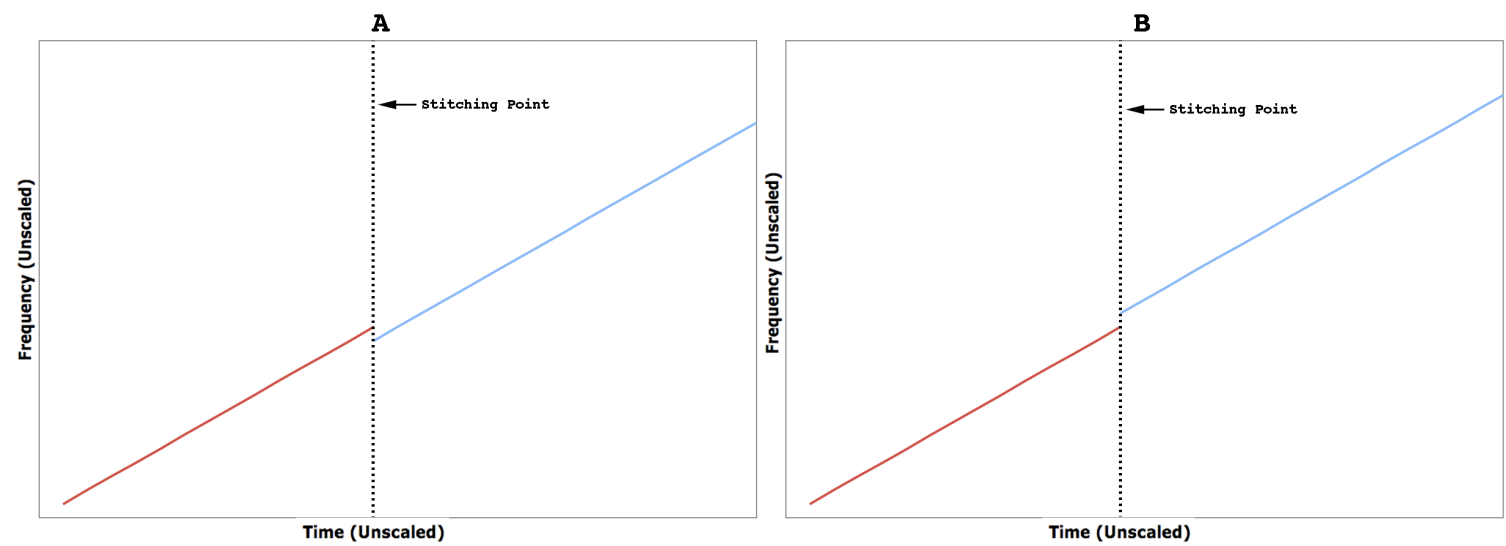

Figure 7.2: Demonstration of two types of stitching errors. The red frequency ramp represents the first continuous tuning segment while the blue frequency ramp stands for the second. The stitching error in image " $A$ " repeats frequency coverage to the frequency ramp. The stitching error in image "B" omits frequency coverage to the frequency ramp.

A $100 \mathrm{pm}$ free spectral range (FSR) interferometer is used to analyze the linearity of the frequency ramp in the time domain, including the stitching errors. Figure (7.3) shows the test setup needed to analyze the frequency ramp using the $100 \mathrm{pm}(12.5 \mathrm{GHz})$ FSR interferometer, a photodiode to convert the laser to an electrical signal, and a HP 54501A Digitizing Oscilloscope. A completely linear ramp, using the test setup in Figure (7.3), would result in a pure sinusoid signal with frequency 3 exahertz $\left(10^{18}\right)$ in the oscilloscope. The distance between to adjacent peaks of the sinusoid represents $100 \mathrm{pm}$ of bandwidth. 


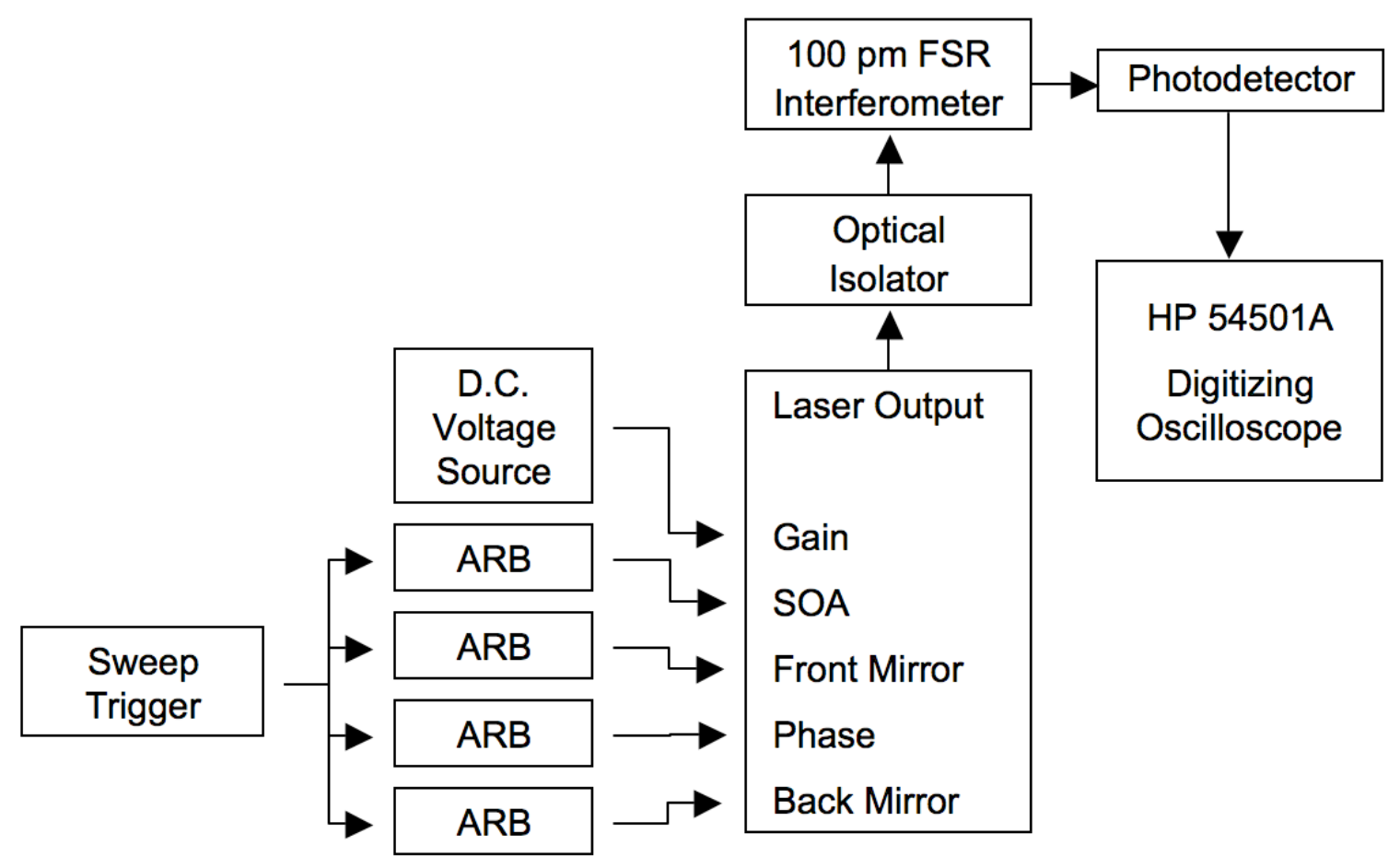

Figure 7.3: Block diagram of the test setup needed to analyze the linearity of frequency ramps.

Running the power leveled linear frequency ramp on the SG-DBR laser and taking the laser output through the $100 \mathrm{pm}$ FSR interferometer, detailed analysis of the stitching errors can be completed. Figure (7.4) and Figure (7.5) show the first stitching error of the L-band SG-DBR power leveled frequency ramp running at $100 \mathrm{~Hz}$ repetition rate. The first stitching error, as well as the other stitching errors in the frequency ramp, repeat frequency coverage as shown in Figure (7.2) Image "a". 


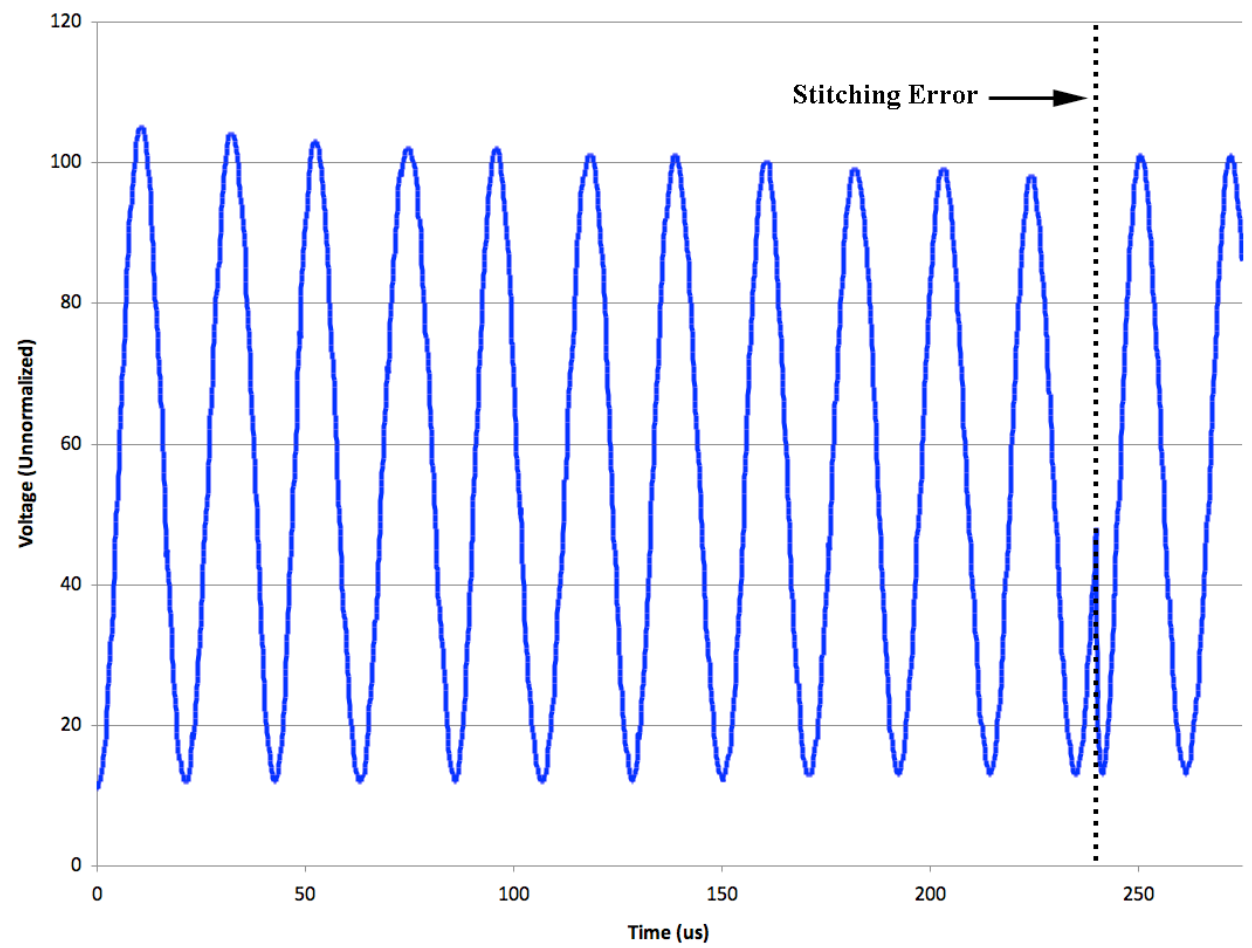

Figure 7.4: Oscilloscope output from $100 \mathrm{pm}$ interferometer showing the first stitching error, which jumps the ramp back in frequency.

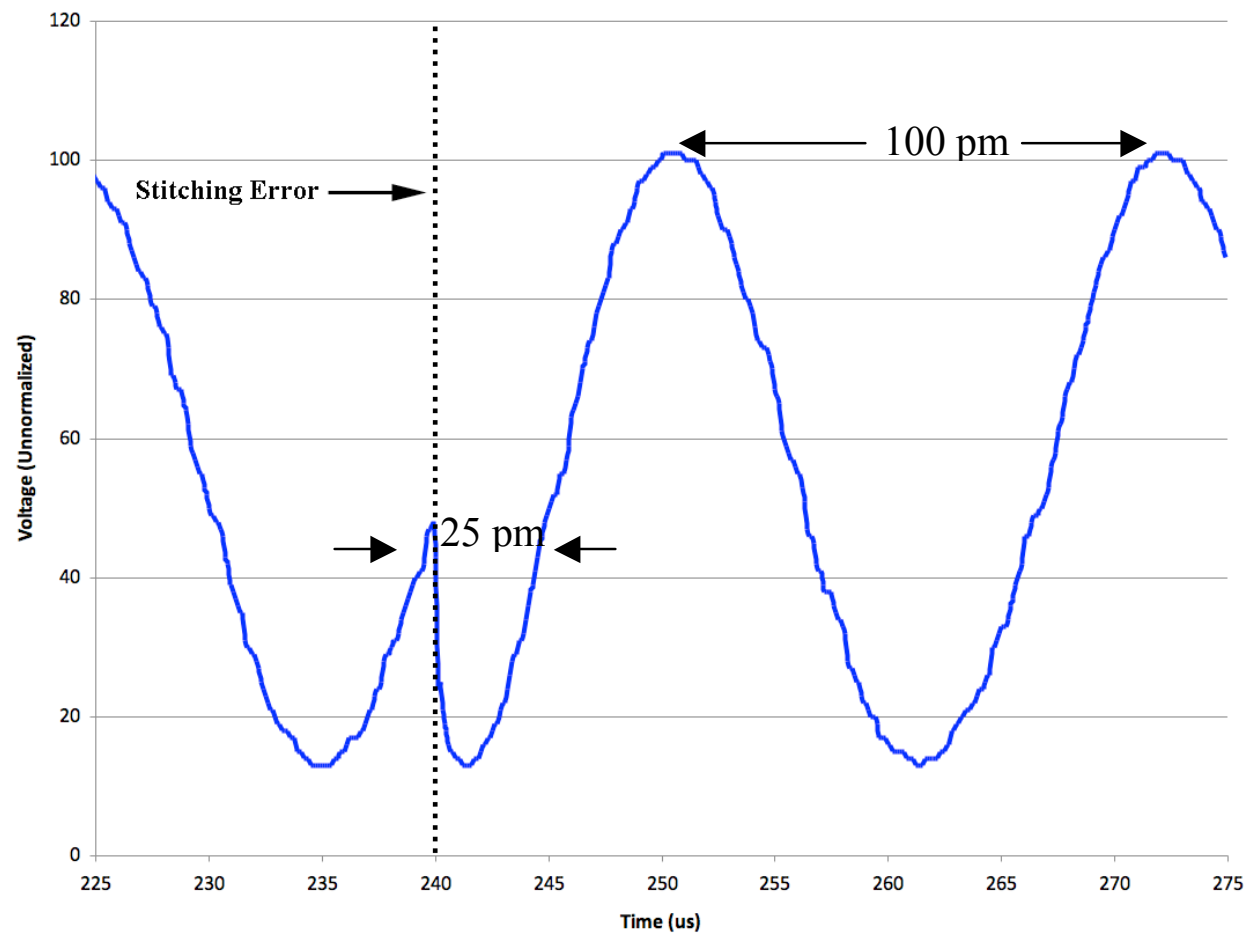

Figure 7.5: Zoomed in plot of oscilloscope output from $100 \mathrm{pm}$ interferometer showing the first stitching error, which jumps the ramp back in frequency. The stitching error repeats approximately 25 pm of wavelength. 


\section{2 - Repairing Stitching Errors}

For slower laser repetition rates, approximately $100 \mathrm{~Hz}$ or less, the method for fixing stitching errors is straightforward. Since the stitching errors of the L-band and Cband power leveled linear frequency ramps always jump backwards in frequency at the stitching point, one can simply delete the extra frequencies. The stitching errors jump back in frequency since the after the stitching error there is additional carrier density and thermal transients left over from before the stitch. The characterization points were chosen at steady state and these transients not present during characterization tend to increase the wavelength of the output frequency which relates to lower frequency. Figure (7.6) and Figure (7.7) demonstrate the fix of the first stitching error of Figure (7.4) and Figure (7.5) respectively. In Figure (7.7) a glitch remains in the waveform due to the 50 ns dead time of the Agilent Arbitrary Waveform Generator.

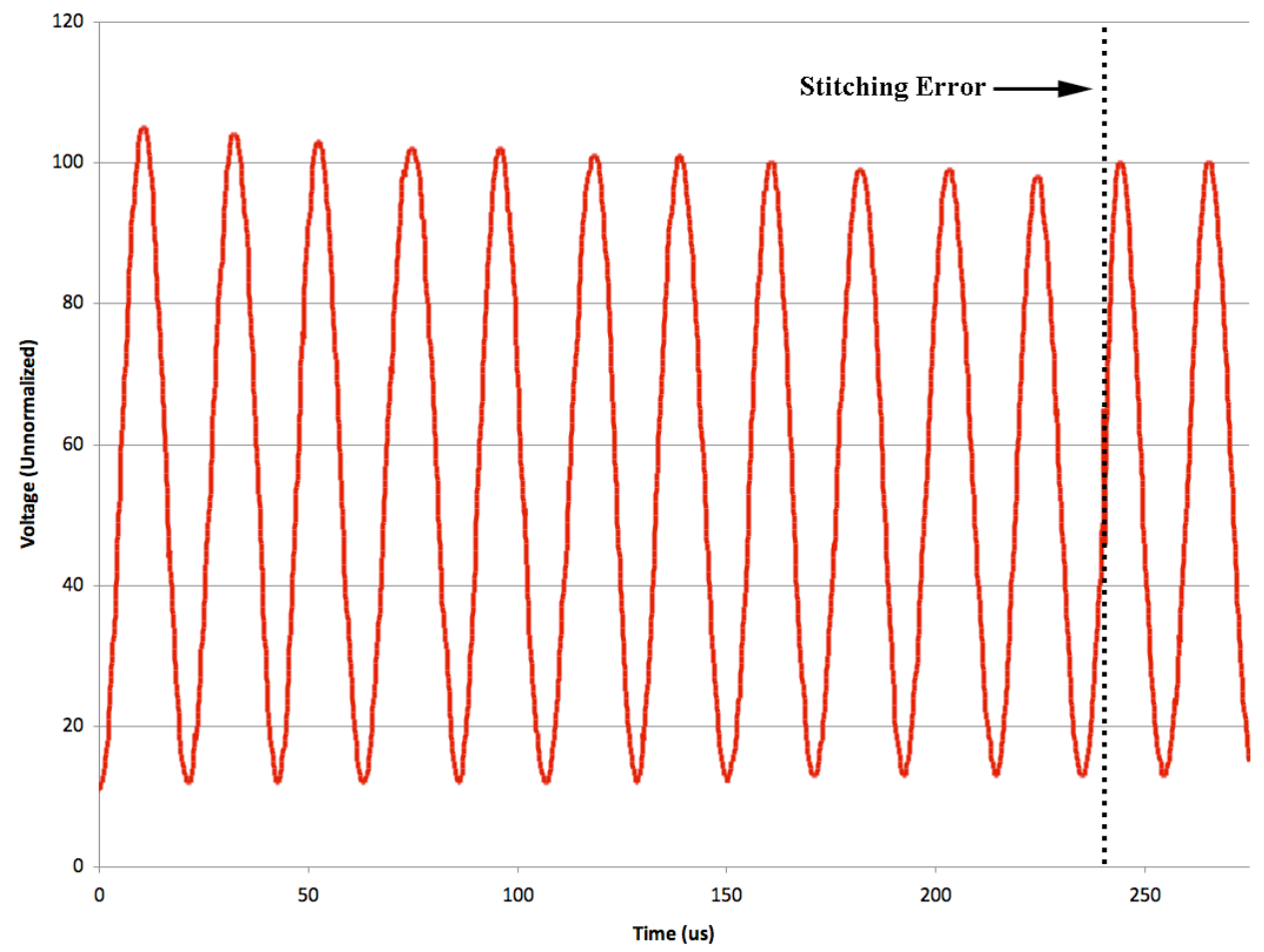

Figure 7.6: First stitch point fixed for the L-band laser trace shown in Figure (7.4) at $100 \mathrm{~Hz}$ repetition rate. 


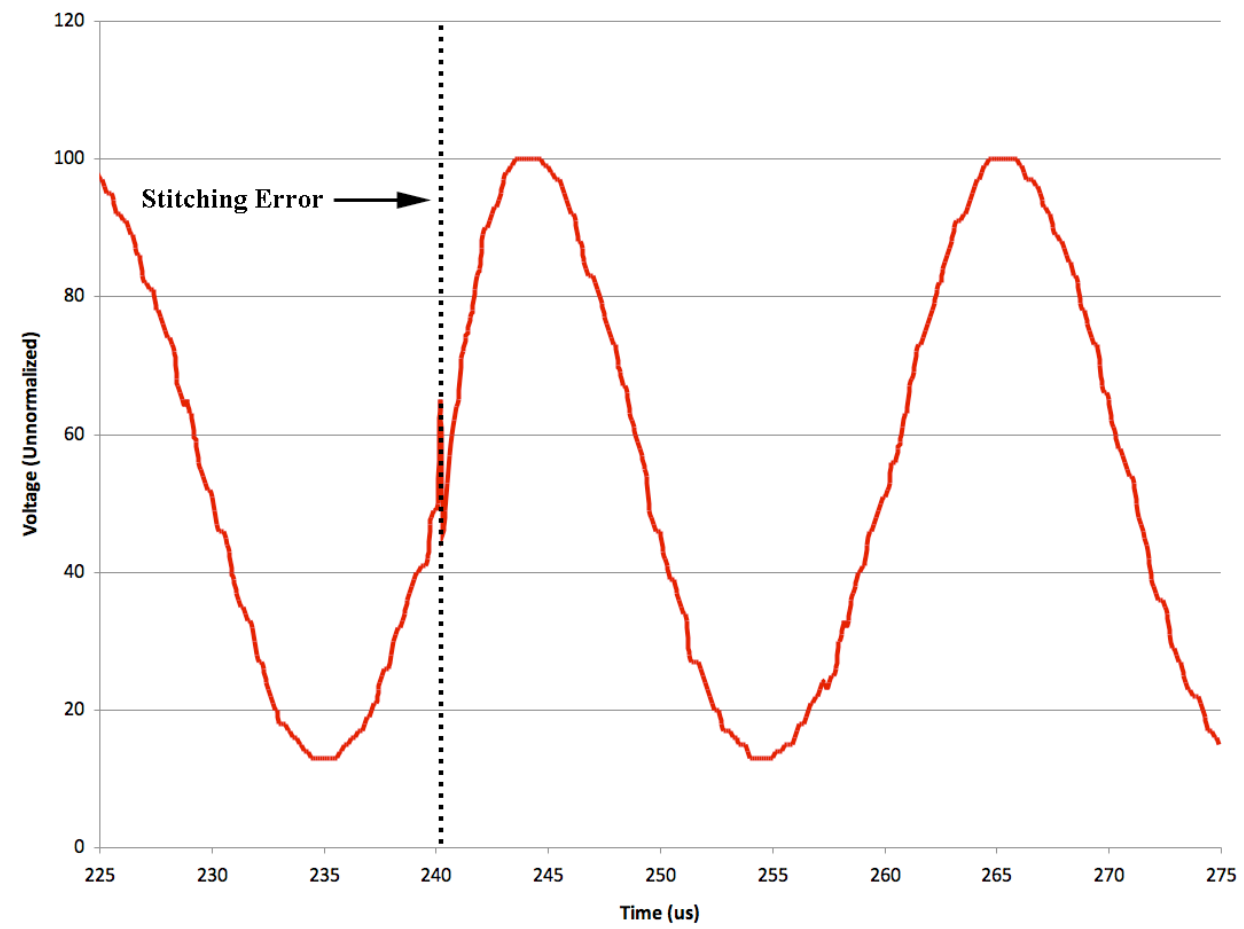

Figure 7.7: Zoomed in plot of first stitch point fixed from the L-band trace shown in Figure (7.5) at a $100 \mathrm{~Hz}$ repetition rate. A glitch from the stitching point is still evident. At the instant of the stitching error the output is undefined.

When the repetition rate increases past one hundred hertz up to $10 \mathrm{kHz}$ and beyond, the $50 \mathrm{~ns}$ dead time of the ARB becomes a large influence on the linearity of the frequency ramp. Figure (7.8) presents similar data to Figure (7.4) and Figure (7.5), only for a $10 \mathrm{kHz}$ repetition rate. 


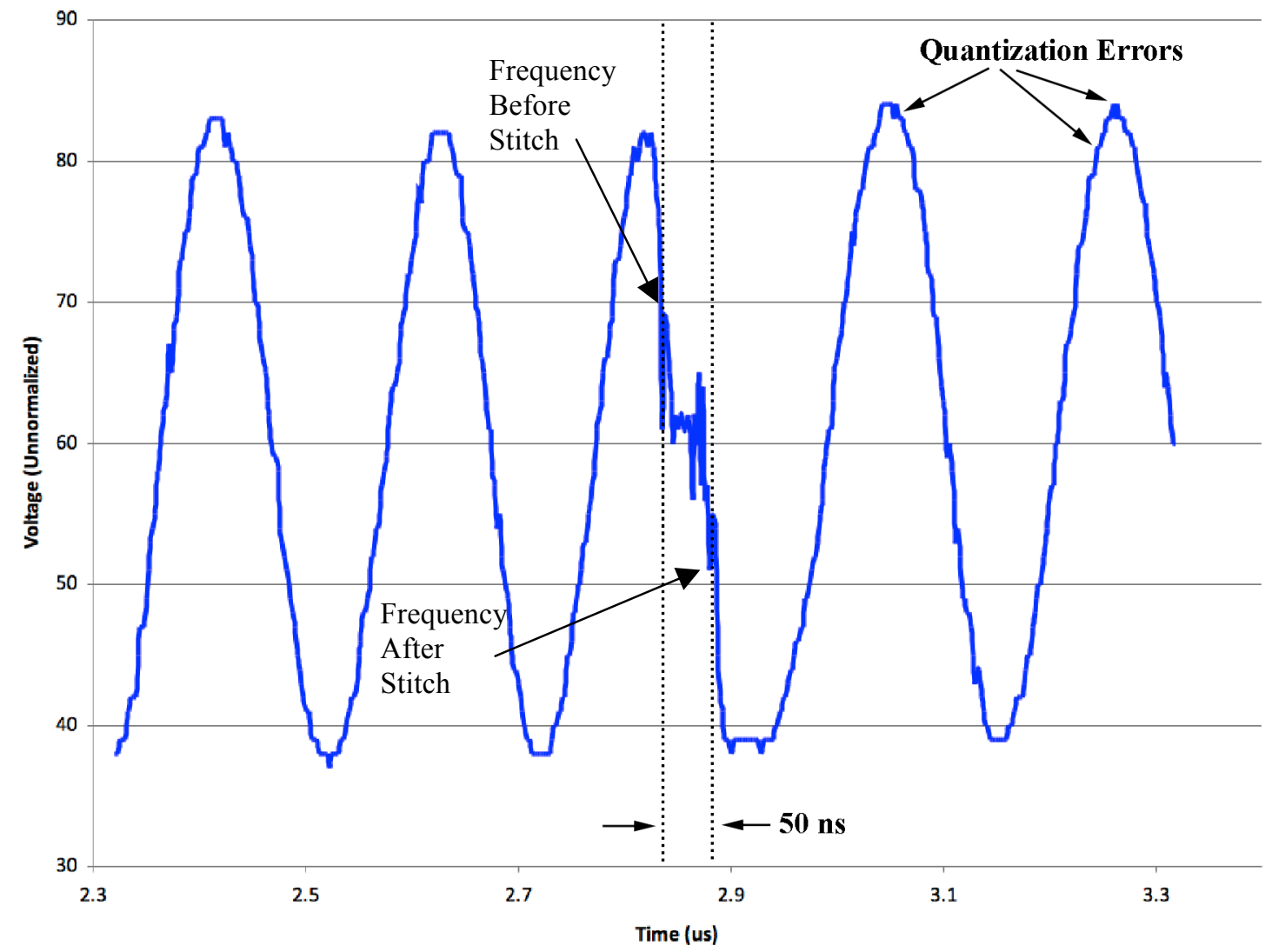

Figure 7.8: Oscilloscope data for the first stitching error in the L-band laser at a $10 \mathrm{kHz}$ repetition rate. $50 \mathrm{~ns}$ of dead time from the ARB produces a period of undefined output shown above. During the $50 \mathrm{~ns}$ shown by the window, the output is undefined. Also shown are examples of the quantization errors, which consist of discrete jumps in the voltage on the oscilloscope and cause broadband noise in FFT measurements.

Higher quality waveform generators with faster rise and fall times would bring the dead time down significantly, but that is beyond the scope of this thesis. Two additional techniques are needed to fix a stitching error at faster repetition rates. The simplest method to deal with the $50 \mathrm{~ns}$ dead time of the ARB, or the $5 \mathrm{~ns}$ dead time of the three tuning sections themselves [11], involves "delaying" the frequency ramp until the ARB and laser has settled into its new wavelength. Once the ARB has finished switching values, the frequency ramp can be continued. Figure (7.9) demonstrates the "delayed" frequency ramp and Figure (7.10) shows an example of the electrical waveforms necessary to "wait" after a stitching point. 


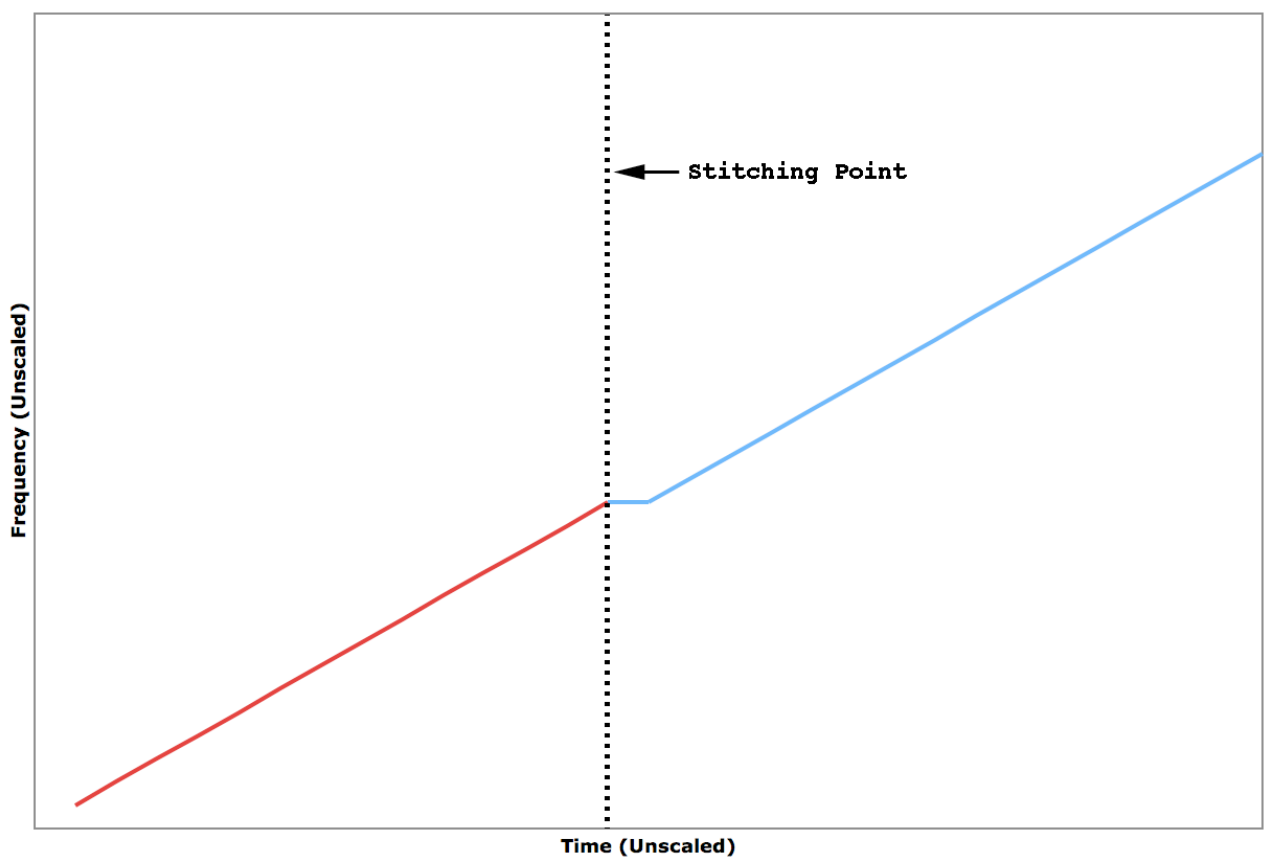

Figure 7.9: Demonstration of delaying the frequency ramp to wait approximately $50 \mathrm{~ns}$ for the ARB values to settle before continuing with the frequency ramp. The red frequency ramp represents the first continuous tuning segment while the blue frequency ramp stands for the second.
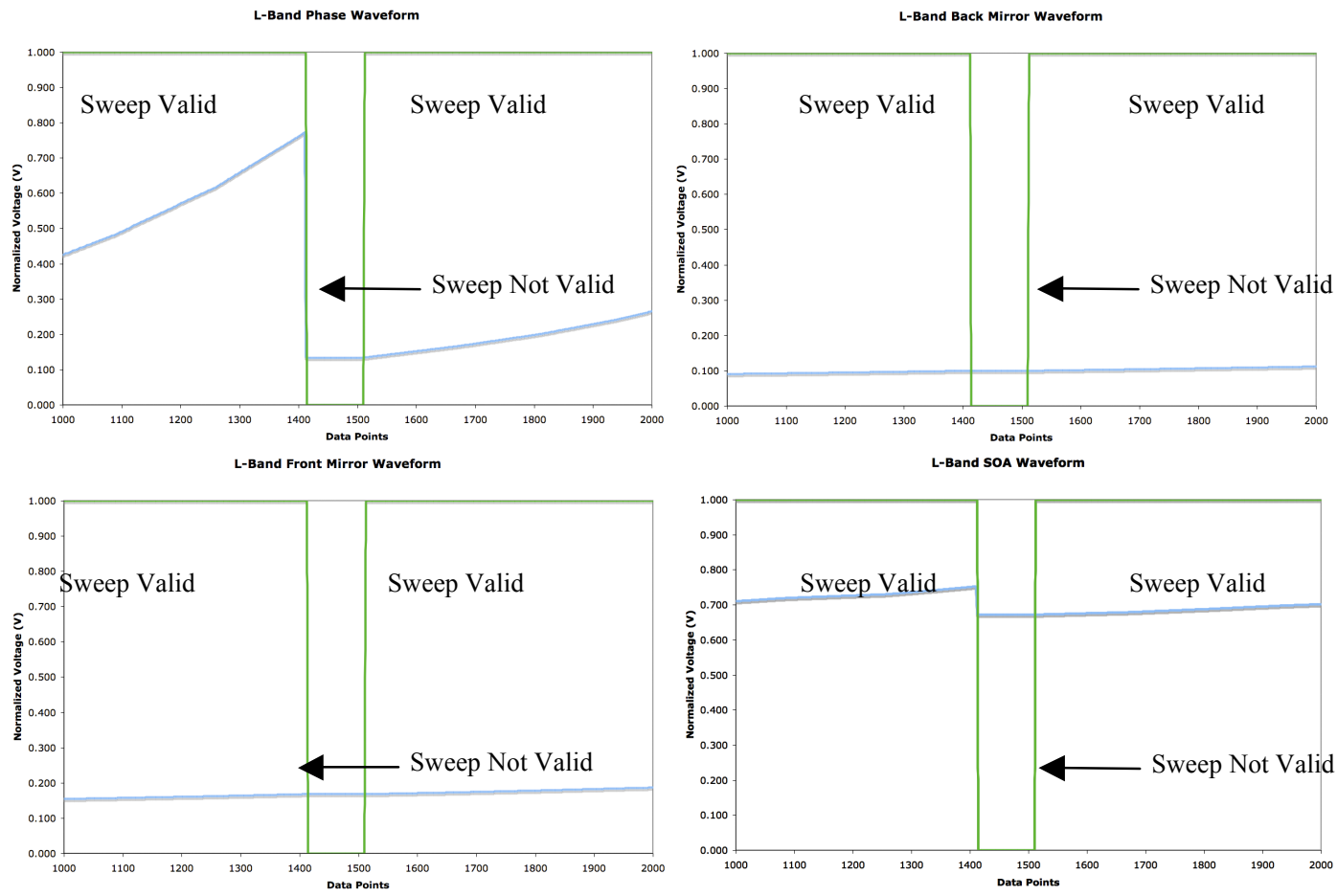

Figure 7.10: Plots of phase, front mirror, back mirror, and SOA waveforms showing the first stitching point and the intentional "delay" after the stitching point. The middle trace shows the section waveform. The notch signal trace is $1 \mathrm{~V}$ when the laser is sweeping frequencies and $0 \mathrm{~V}$ when the laser sweep is delayed, letting the ARBs settle. 
The power leveled linear frequency ramp laser output will be fed into D/A converters, in the case of OCT measurements, which will perform the FFT on the complete laser ramp. To correctly use the data, the system will need to know when the laser was ramping through frequencies and when the laser was being "delayed." Therefore, a validity signal needs to be created that will instruct the system to discard the information when the laser ramp is "delayed." The "green" traces in Figure (7.10) show the validity signal for the linear frequency ramp. The convention chosen for the validity signal is $1 \mathrm{~V}$ for "sweeping" and $0 \mathrm{~V}$ for "delayed."

Putting the first low repetition rate stitch fix technique with delayed waveforms and adding the validity signal, gives the signal shown in Figure (7.11) and Figure (7.12) is obtained. As in the case shown in Figure (7.6) and Figure (7.7), the first stitching point is analyzed and fixed (but at $10 \mathrm{kHz}$ repetition rate). 


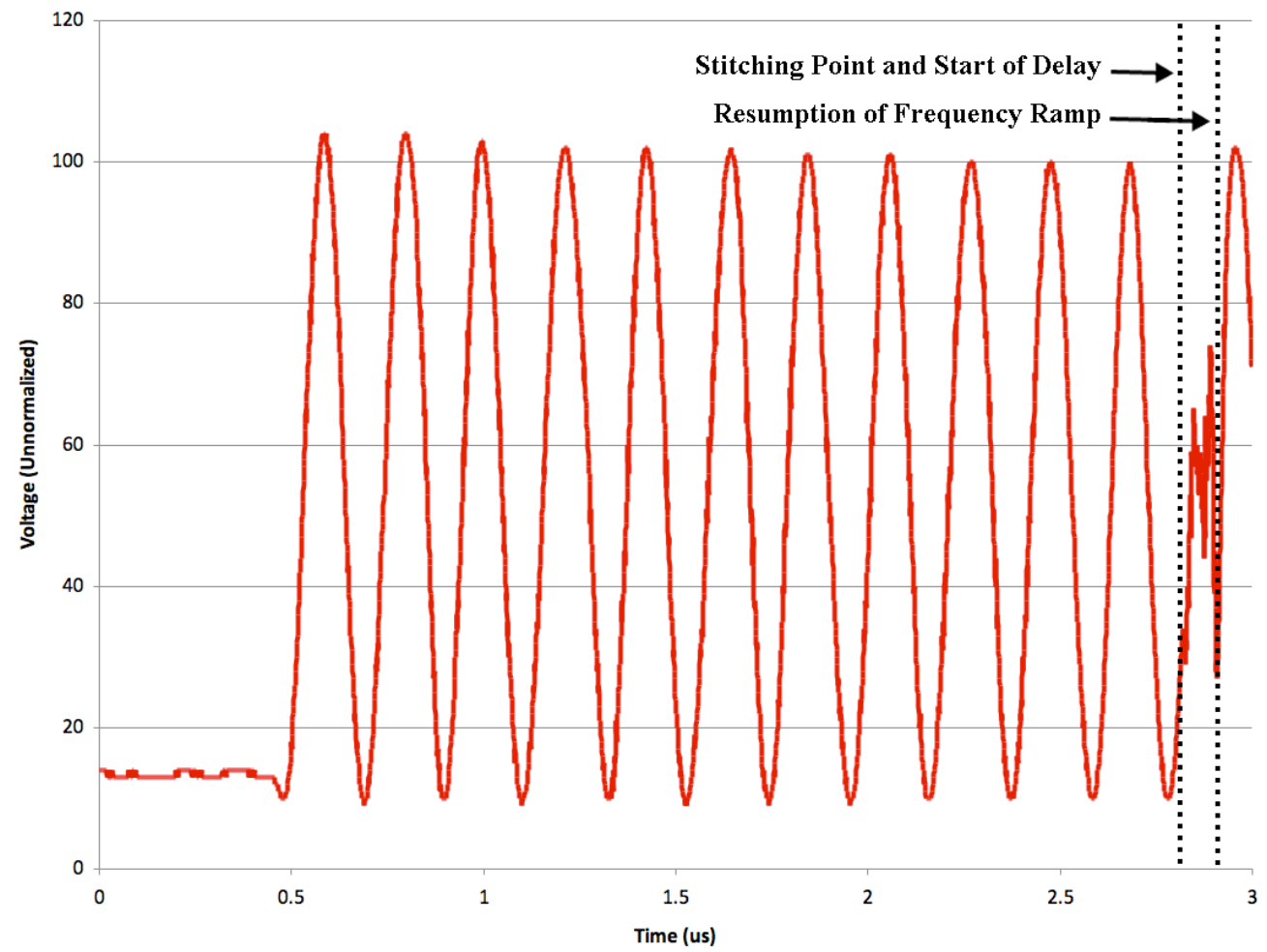

Figure 7.11: First stitching point for the L-band laser (originally shown in Figure (7.8)) delayed during the dead time of the ARB and fixed at $10 \mathrm{kHz}$ repetition rate.

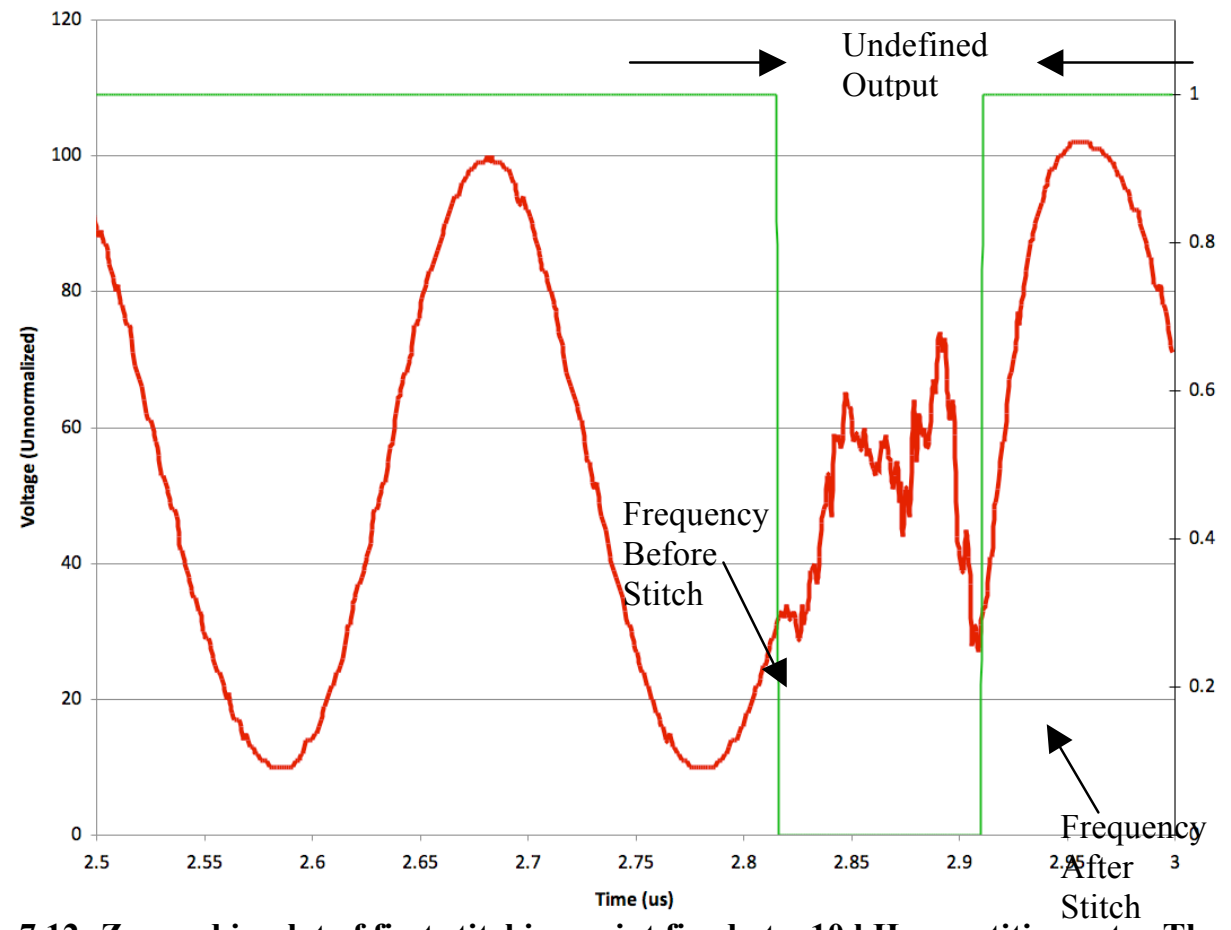

Figure 7.12: Zoomed in plot of first stitching point fixed at a $10 \mathrm{kHz}$ repetition rate. The sweep pauses until the ARBs settle and then the sweep begins again where it left off. Also, a validity signal lets the system know when to ignore the laser output. 
A final method for combating stitching errors involves pre-emphasis on the ARB voltages to force these transients to settle faster. By overshooting the destination current for a set time before switching to the final section current, tuning times less than $5 \mathrm{~ns}$ can be attained [11]. However, pre-emphasis techniques are beyond the scope of this thesis since our ARBs have tuning times of approximately $50 \mathrm{~ns}$.

The methods outlined in Section 5.2 for reducing the stitching errors during the frequency ramp with not be implemented in this thesis for reducing all the stitching errors in a linear frequency ramp. A test setup needed to effectively fix the approximately 50 stitching errors in a full $50 \mathrm{~nm}$ linear frequency ramp requires its own research.

\section{3 - Stability of Stitching Errors}

Section 7.2 showed that the stitching errors present in the linear frequency ramps could be drastically reduced. However, the stability of the stitching error fixes needs to be determined. The stability of a stitching error fix represents how much the fix changes as time passes. The frequency ramp with the first stitch fixed, shown in Figure (7.6) and Figure (7.7), was analyzed for the stability of the fix. Using the same test setup as Figure (7.3), three traces were downloaded. One trace was taken immediately after the first stitch was fixed, for the second trace the laser sweep was turned off and restarted, and the third trace was taken after the laser sweep was left on for 19 hours. Figure (7.13) combines these three traces for the L-band power leveled linear frequency ramp. 


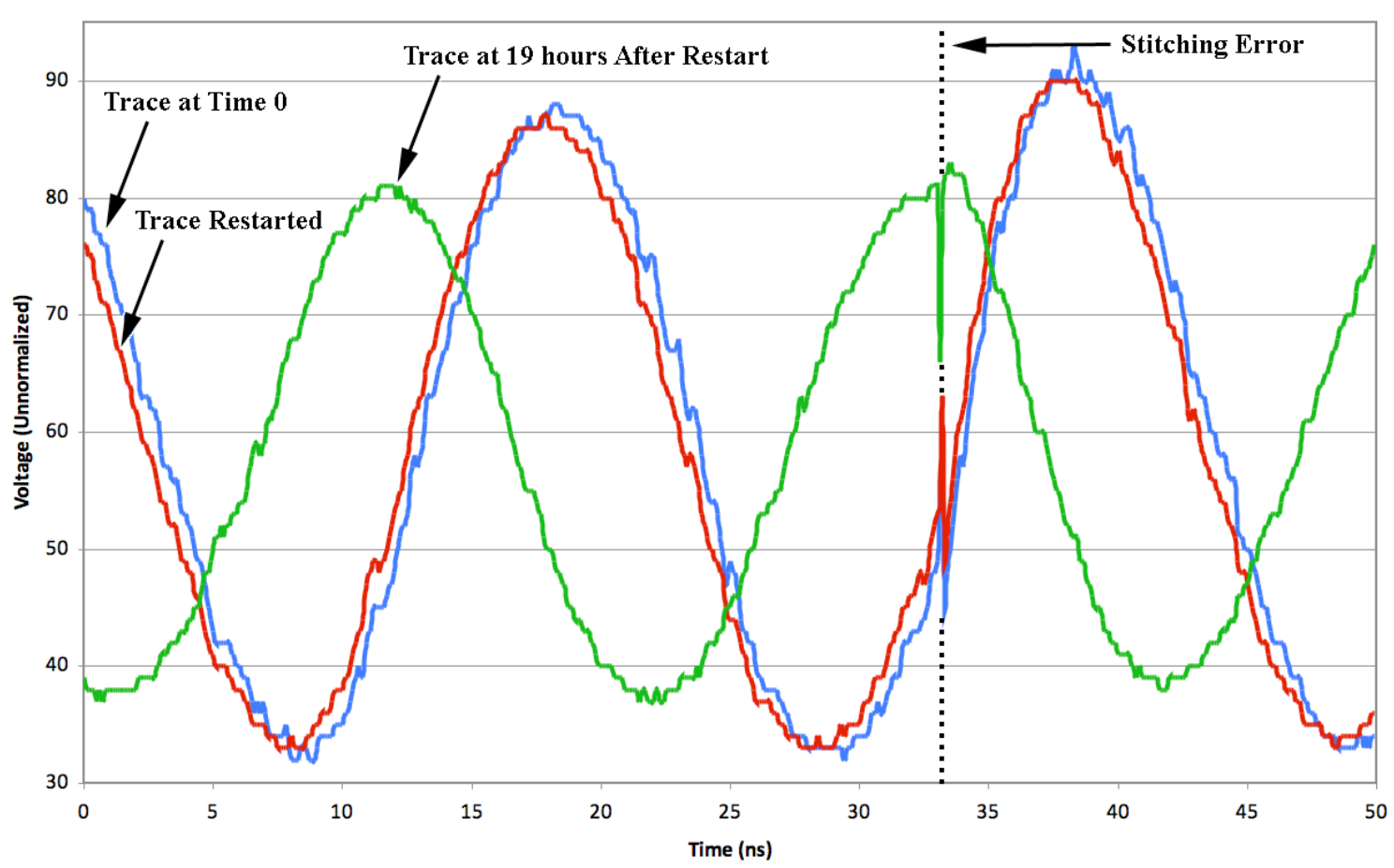

Figure 7.13: Stability test for the first stitching error of the L-band power leveled linear frequency ramp. Note that the stitches stay relatively stable even though the absolute frequency of the frequency ramp shifts by approximately 25 pm after 19 hours.

It can be seen in Figure (7.13), that the stitching error does not change much with time. Even though the position and size of the stitching error does not change, the absolute wavelength that the stitching error occurs at does change by approximately 25 pm after 19 hours. This could be due to numerous sources such as a temperature difference in the ARB or TEC. However, since the entire frequency ramp is shifted by 25 pm, this effect is negligible.

\section{4 - Physical Limitations on Stitching Errors}

One of the primary attributes of the SG-DBR laser is the small switching time, which will allow fast sweep rates. Modeling the front mirror, back mirror, phase, and SOA ports is necessary to determine the high frequency behavior of the SG-DBR laser. This will give the minimum stitching error time gap. The gain section is not modeled 
since only D.C. currents are applied. As stated previously, each primary section in the SG-DBR laser consists of a PIN diode [3]. A parallel resistor-capacitor (RC) circuit provides a simple model for a PIN diode. However, a series inductor must also be added to the parallel RC model. The butterfly pins of the SG-DBR laser package will add a series inductance of approximately 15 to $20 \mathrm{nH}$, since a small wire with a relatively large distance over a ground plane will cause a series inductance [12]. The final RLC circuit model used for this laser is shown in Figure (7.14).

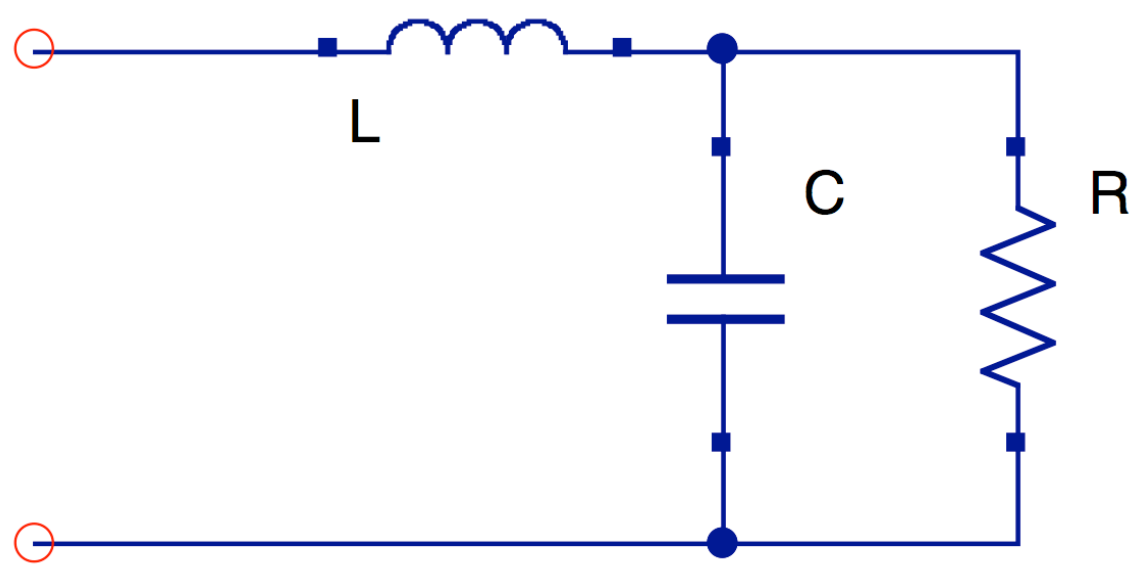

Figure 7.14: RLC model used for each section on the SG-DBR laser.

To model this RLC circuit, a vector network analyzer (VNA) will compute the port impedance for each of the sections at 300 increments from $10 \mathrm{MHz}$ to $3 \mathrm{GHz}$. Testing each port is performed without the section properly biased and another with $3 \mathrm{~V}$ (approximately $30 \mathrm{~mA}$ ) from a D.C. power supply inputted to the "port bias" on the VNA. Figure (7.15) shows the laser port impedance test setup. 


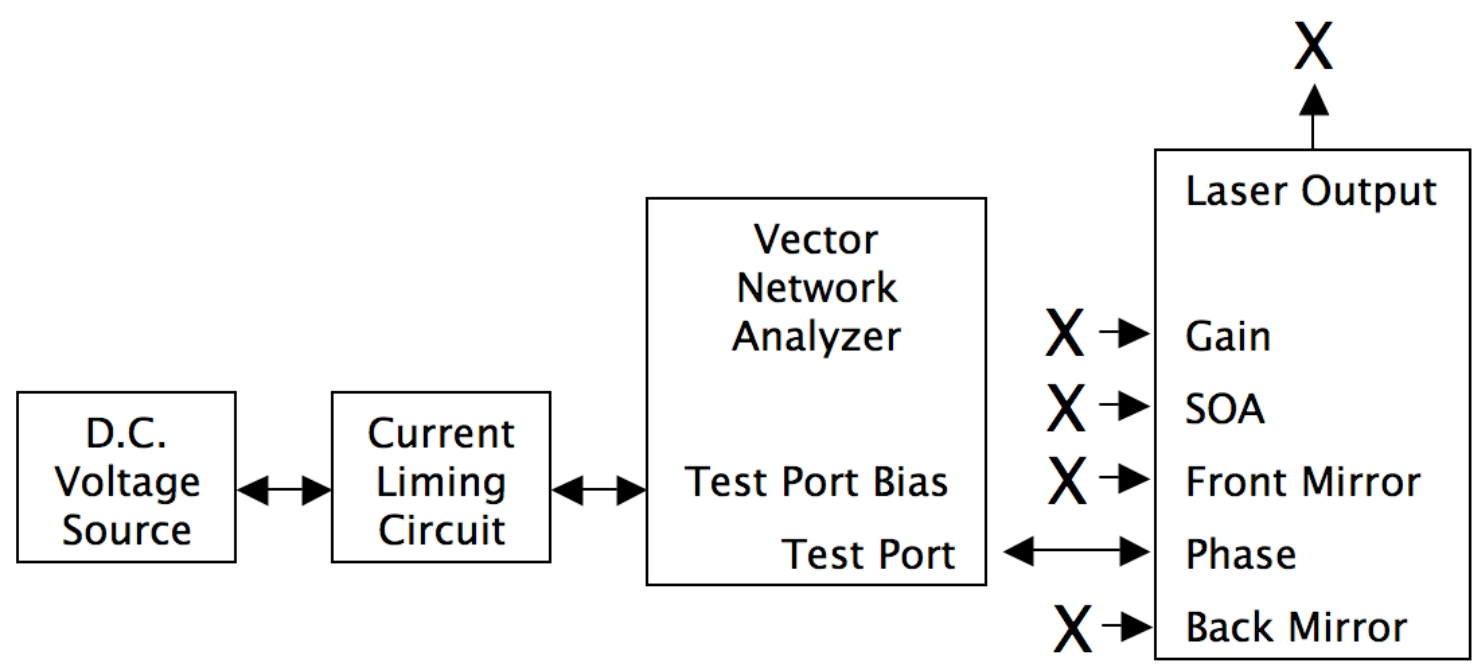

Figure 7.15: Port impedance test setup. VNA bias-tee connection and a current source can bias the section plugged into the test port while performing impedance test. Test port is plugged into front mirror, back mirror, phase, and SOA sections to record port impedances with D.C. voltage source ON and OFF. Current limiting circuits associated with the port being tested are also used. All ports not currently under test, including the laser output, are left "open."

Four measurements are downloaded from the VNA using the setup in Figure (7.15) for each port. A swept frequency port impedance test from $10 \mathrm{MHz}$ to $3 \mathrm{GHz}$ and a time domain reflectometry (TDR) test are both performed with the SG-DBR laser section port biased "on" and "off." Figure (7.16) and Figure (7.17) are examples of swept frequency port impedance plots of the phase section of the L-band laser biased “on" and "off” respectably. Figure (7.18) and Figure (7.19) are TDR measurement plots of the phase section of the L-band laser biased "on" and "off” respectably. 


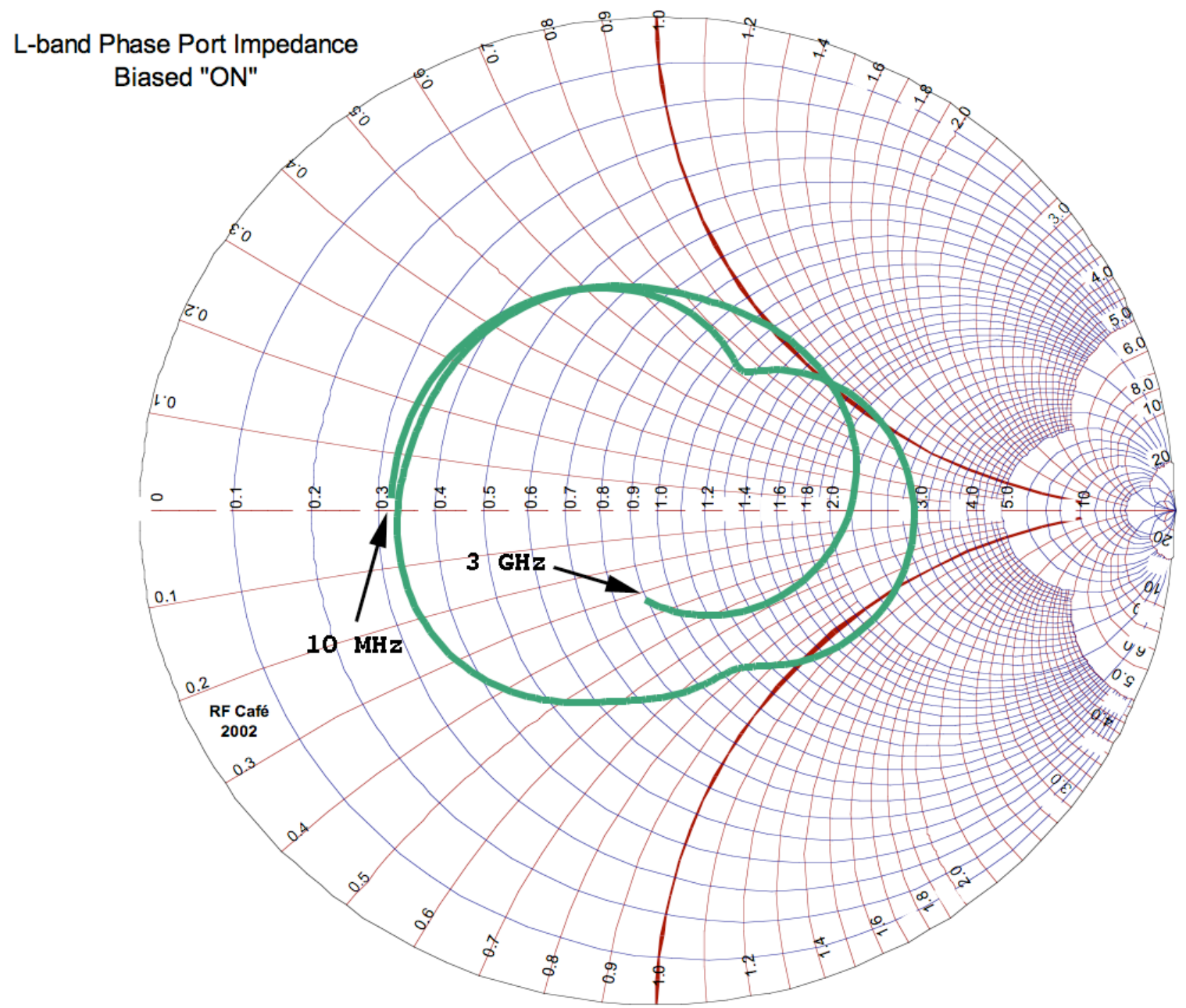

Figure 7.16: Swept frequency VNA port impedance plot for L-band phase section biased "ON." 


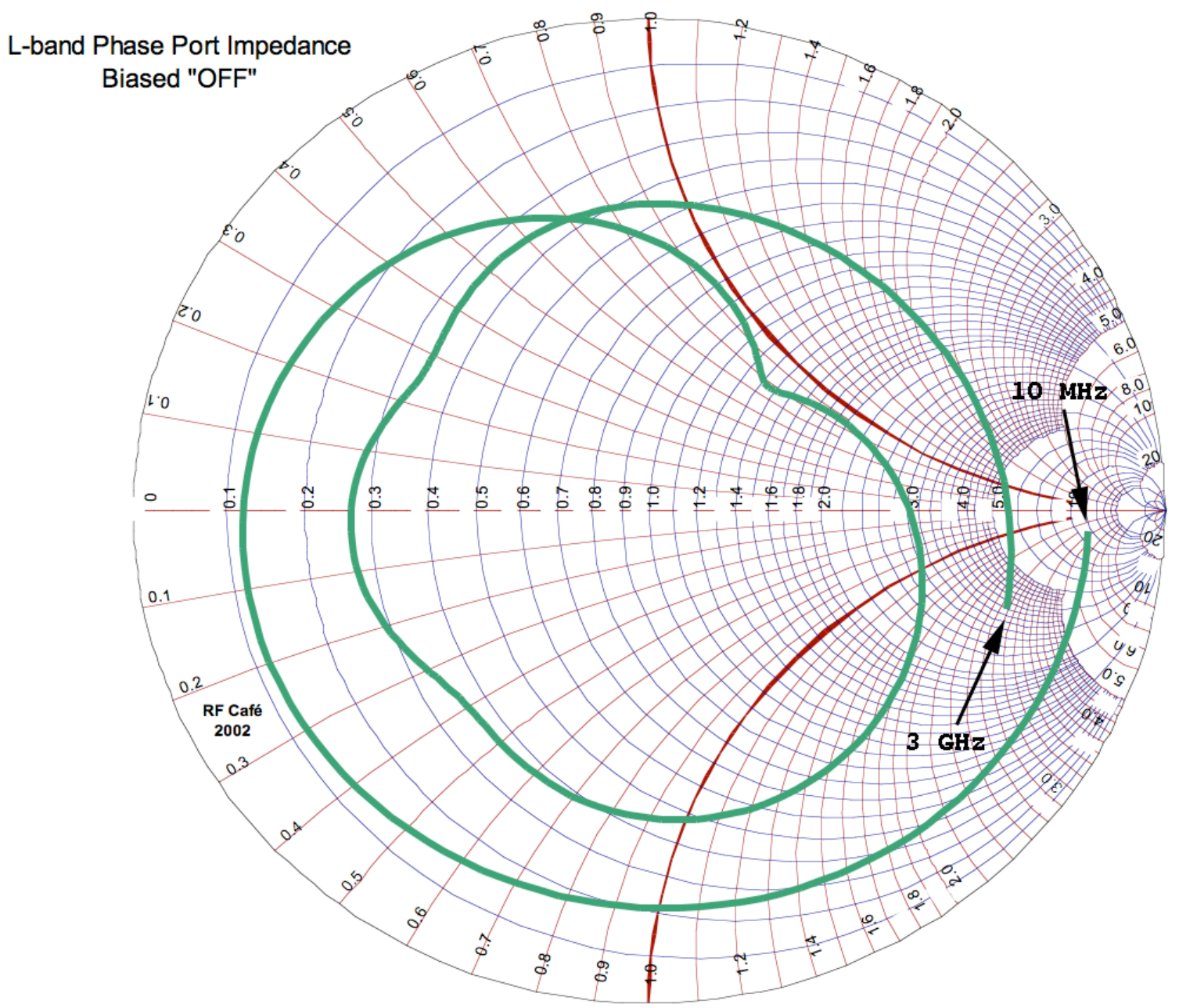

Figure 7.17: Swept frequency VNA port impedance plot for L-band phase section biased "OFF." 
TDR Measurement L-Band Phase Biased "ON"

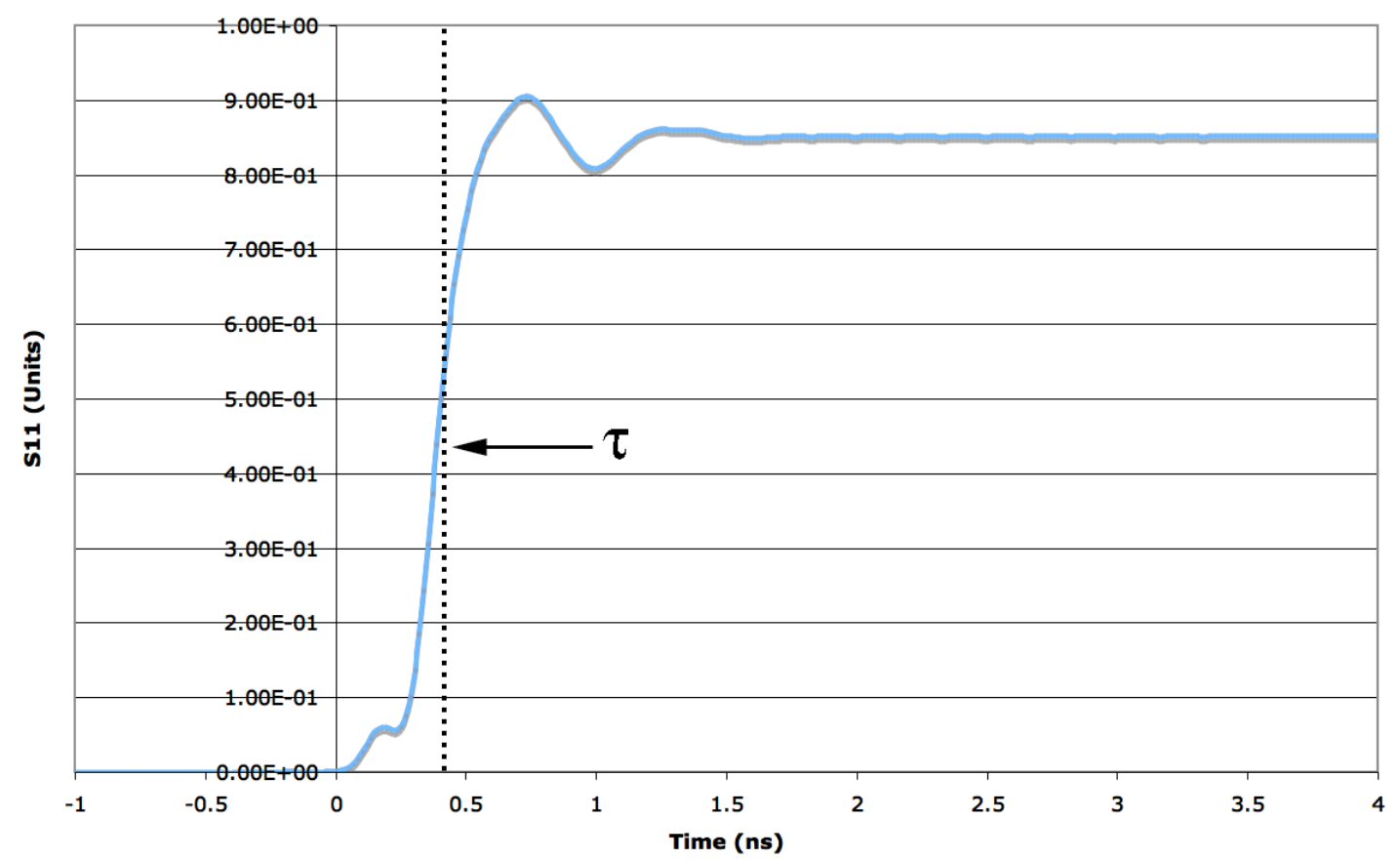

Figure 7.18: VNA TDR measurement data for L-band phase section biased "ON." Also shown is the $\mathrm{RC}$ time constant point $\tau$ (see following paragraph for more information).

TDR Measurement L-Band Phase Biased "OFF"

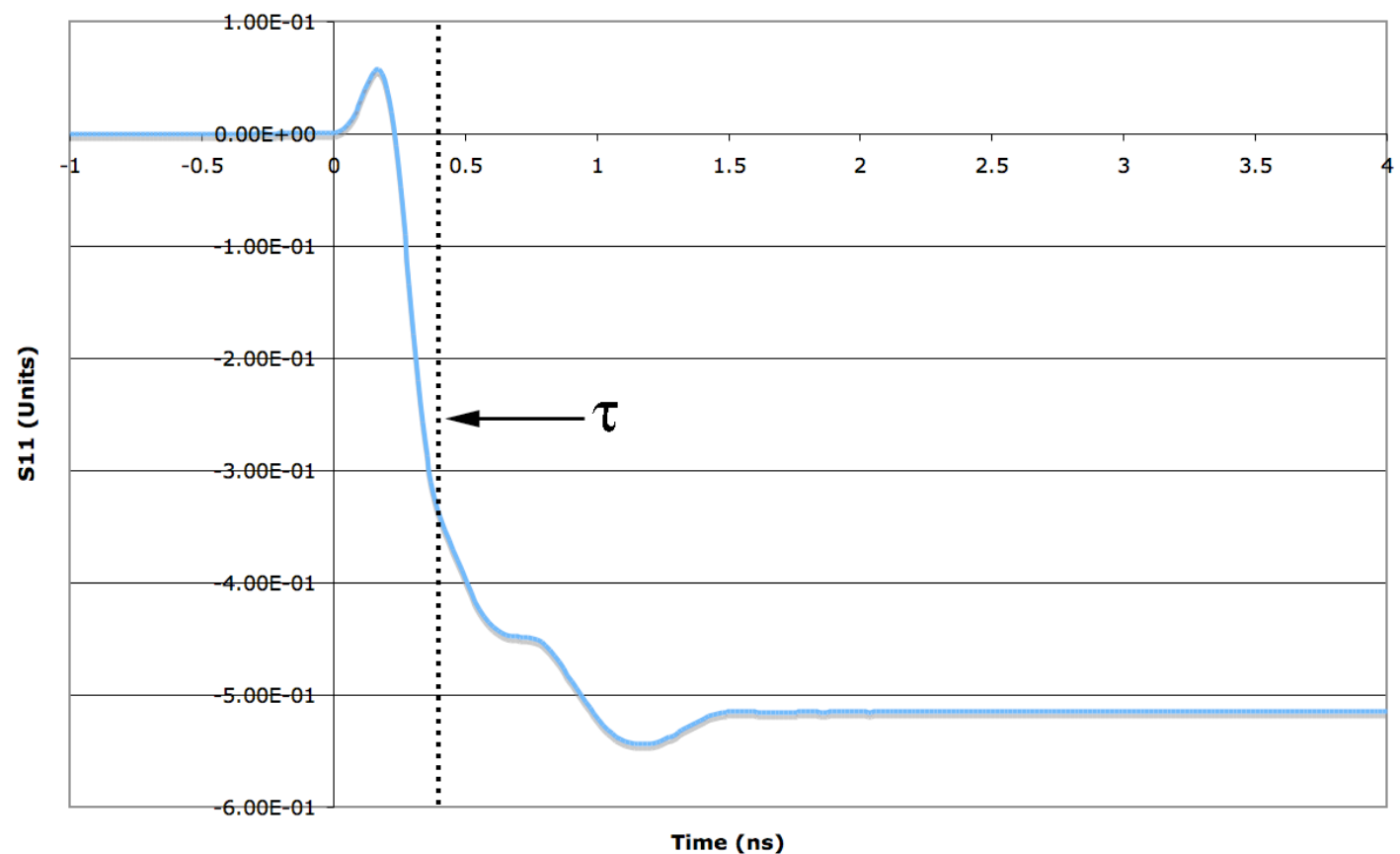

Figure 7.19: VNA TDR measurement data for L-band phase section biased "OFF." Also shown is the RC time constant point $\tau$ (see following paragraph for more information). 
The first model component to calculate from the data is the resistor, R. A small signal TDR measurement, in step function mode, performed from the VNA data gives S11 reflection data versus time. As time approaches infinity in the TDR measurement, the value of $\mathrm{S} 11$ will tend to a value $\mathrm{S} 11_{\text {final }}$. The resistance, $\mathrm{R}$, of the $\mathrm{RLC}$ model shown in Figure (7.14) can be found from solving:

$$
S 11_{\text {final }}=\frac{R-50}{R+50}
$$

The value of $\tau$ is found when S11, in the TDR measurement, reaches $63.2 \%$ of $\mathrm{S} 11_{\text {final }}$. The capacitance, $\mathrm{C}$, can then be found by:

$$
\tau=R C
$$

The series inductance can be found, when the section is biased ON with 3 V D.C., from the value of the reactance at $100 \mathrm{MHz}$ and the equation:

$$
L=\frac{X}{2 \pi 100 M H z}
$$

where $\mathrm{X}$ is the reactance at $100 \mathrm{MHz}$. Finally the cutoff frequency, $f_{\mathrm{c}}$, is found from:

$$
f_{c}=\frac{1}{2 \pi \tau}
$$

Table (7.1) and Table (7.2) give the values for the parameters found for the L-band and C-band lasers respectfully. 


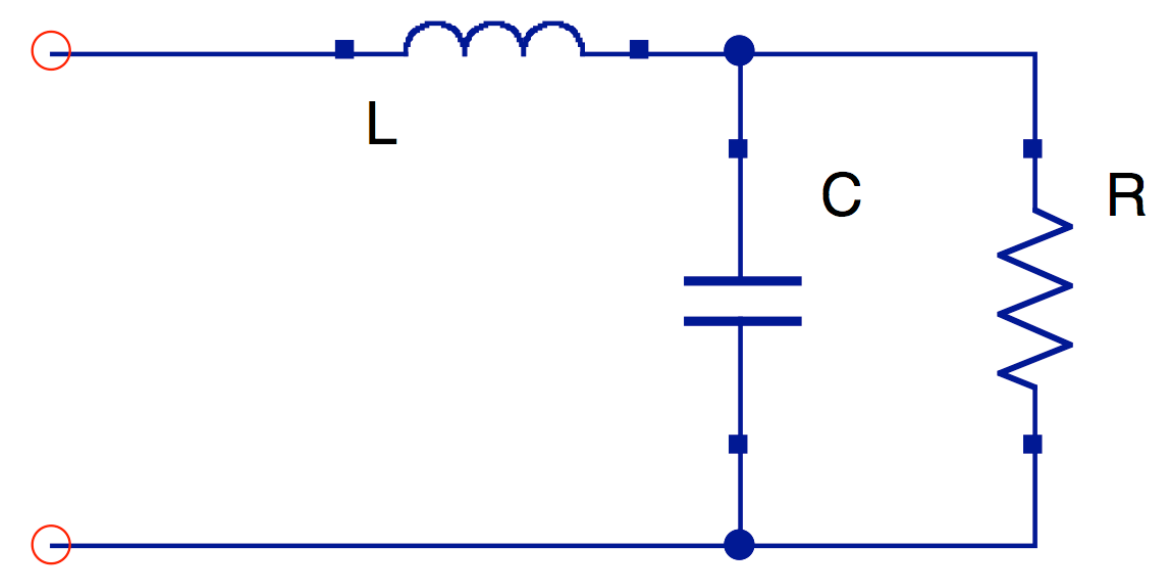

Figure 7.14: RLC model circuit shown again for quick reference for Table (7.1) and Table (7.2).

\begin{tabular}{|c|c|c|c|c|c|c|c|c|}
\hline & \multicolumn{8}{|c|}{ L-Band SG-DBR Laser Port Impedance Modeling } \\
\hline & \multicolumn{4}{|c|}{ Ports Biased "ON" with $\sim 30 \mathrm{~mA}$} & \multicolumn{4}{|c|}{ Ports Biased "OFF" } \\
\hline & FM & $\mathbf{B M}$ & Phase & SOA & FM & $\mathbf{B M}$ & Phase & SOA \\
\hline $\mathbf{R}(\Omega)$ & 7.903 & 3.670 & 16.01 & 3.416 & 731.6 & 444.7 & 626.0 & 546.3 \\
\hline $\mathrm{C}(\mathrm{pF})$ & 49.05 & 91.92 & 24.09 & 111.4 & 0.6990 & 1.312 & 0.6630 & 1.067 \\
\hline L (nH) & 21.40 & 18.55 & 19.58 & 21.17 & 21.40 & 18.55 & 19.58 & 21.17 \\
\hline$\tau(\mathrm{ns})$ & 0.3876 & $\mathbf{0 . 3 3 7 6}$ & $\mathbf{0 . 3 8 5 7}$ & 0.3806 & 0.5113 & 0.5833 & 0.4148 & 0.5828 \\
\hline$f_{\mathrm{c}}(\mathrm{MHz})$ & 410.6 & 471.4 & 412.6 & 418.1 & 311.3 & 272.9 & 283.6 & 273.1 \\
\hline
\end{tabular}

Table 7.1: L-band port impedance test data for four of the primary sections in ON and OFF modes.

\begin{tabular}{|c|c|c|c|c|c|c|c|c|}
\hline \multirow{2}{*}{} & \multicolumn{8}{|c|}{ C-Band SG-DBR Laser Port Impedance Modeling } \\
\cline { 2 - 9 } & \multicolumn{2}{|c|}{ Ports Biased “ON" with $\sim$ 30 $\mathbf{~ A ~}$} & \multicolumn{4}{c|}{ Ports Biased “OFF" } \\
\cline { 2 - 9 } & FM & BM & Phase & SOA & FM & BM & Phase & SOA \\
\hline R $(\boldsymbol{\Omega})$ & $\mathbf{7 . 3 3 3}$ & $\mathbf{5 . 3 4 0}$ & $\mathbf{1 6 . 6 5}$ & $\mathbf{3 . 9 6 5}$ & $\mathbf{5 8 8 . 2}$ & $\mathbf{2 5 5 . 1}$ & $\mathbf{1 2 0 3}$ & $\mathbf{5 8 5 . 5}$ \\
\hline $\mathbf{C}(\mathbf{p F})$ & 47.58 & $\mathbf{5 6 . 9 5}$ & $\mathbf{1 8 . 2 5}$ & $\mathbf{1 1 7 . 4}$ & $\mathbf{0 . 9 5 7 0}$ & $\mathbf{2 . 9 1 8}$ & $\mathbf{0 . 3 1 6}$ & $\mathbf{0 . 9 9 2}$ \\
\hline $\mathbf{L}(\mathbf{n H})$ & $\mathbf{1 8 . 9 2}$ & $\mathbf{1 6 . 1 6}$ & $\mathbf{1 5 . 8 5}$ & $\mathbf{1 9 . 8 2}$ & $\mathbf{1 8 . 9 2}$ & $\mathbf{1 6 . 1 6}$ & $\mathbf{1 5 . 8 5}$ & $\mathbf{1 9 . 8 2}$ \\
\hline$\tau(\mathbf{n s})$ & $\mathbf{0 . 3 4 8 9}$ & $\mathbf{0 . 3 0 4 1}$ & $\mathbf{0 . 3 0 4 0}$ & $\mathbf{0 . 4 6 5 4}$ & $\mathbf{0 . 5 6 3 0}$ & $\mathbf{0 . 7 4 4 3}$ & $\mathbf{0 . 3 8 1 3}$ & $\mathbf{0 . 5 8 0 6}$ \\
\hline$f_{\mathbf{c}}(\mathbf{M H z})$ & $\mathbf{4 5 6 . 1}$ & $\mathbf{5 2 3 . 3}$ & $\mathbf{5 2 3 . 5}$ & $\mathbf{3 4 1 . 9}$ & $\mathbf{2 8 2 . 7}$ & $\mathbf{2 1 3 . 8}$ & $\mathbf{4 1 7 . 4}$ & $\mathbf{2 7 4 . 2}$ \\
\hline
\end{tabular}

Table 7.2: C-band port impedance test data for four of the primary sections in ON and OFF modes.

From $\tau$, one can in theory, electrically tune the laser faster than 1 ns. However, the SG-DBR laser tuning times are limited by parasitic thermal time constants resulted from the switching and are limited to approximately $5 \mathrm{~ns}$ [11]. Also, the VNA measurements are small signal, meaning one can expect the time constants to rise significantly for large signal tuning. Figure (7.19) demonstrates how the thermal transients affect the laser tuning times more than the electrical tuning times. 


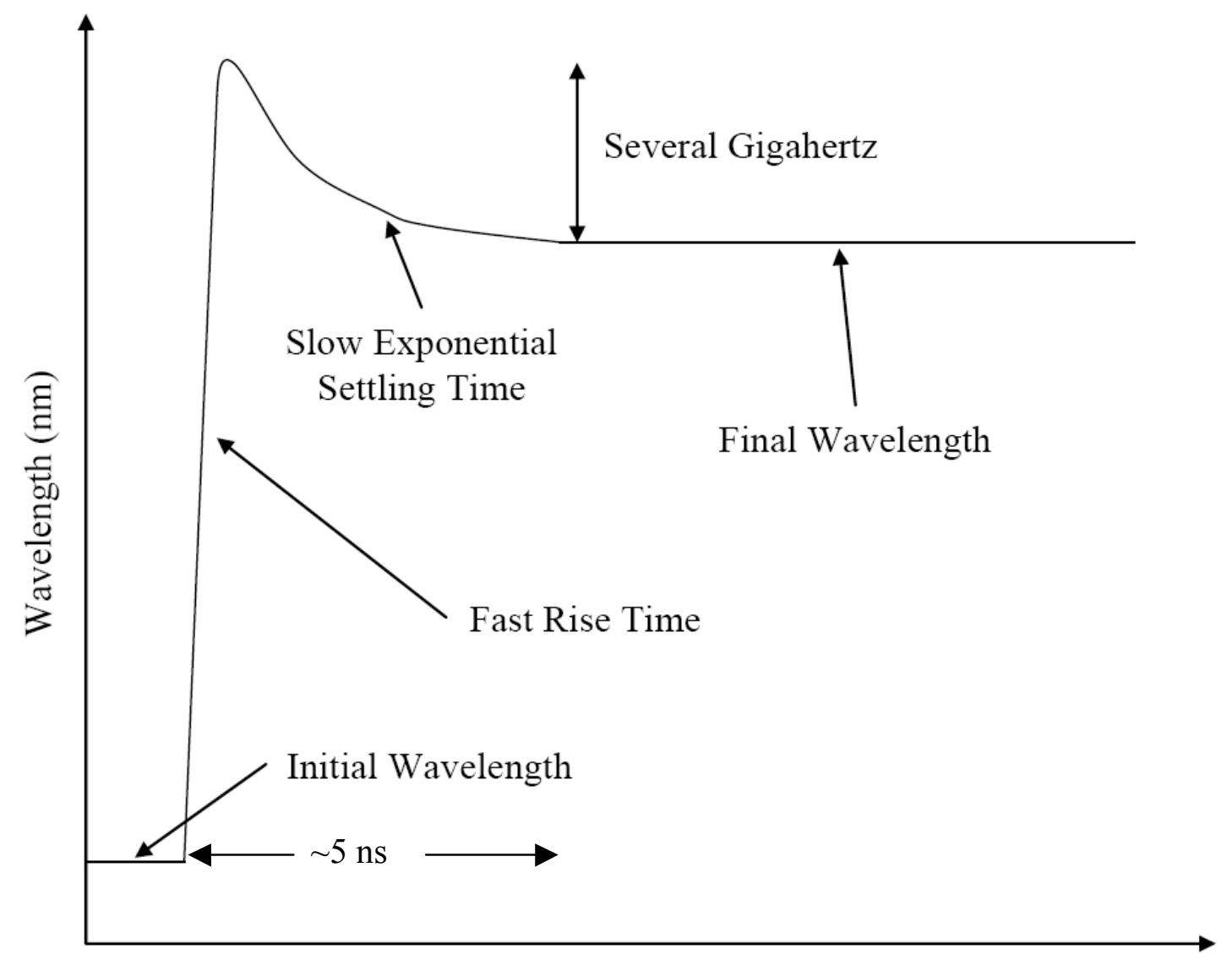

Time (ns)

Figure 7.20: Visual representation of wavelength tuning versus time. Fast rise time is dominated by the electrical characteristics of the SG-DBR laser. Slow exponential settling time is due to the thermal time constants in the laser.

With the approximately $5 \mathrm{~ns}$ of dead time caused by the thermal transients of the laser, completely removing stitching errors is not possible. However, the $5 \mathrm{~ns}$ of dead time needed for the thermal transients to settle can be dealt with in the way shown in Figure (7.12) by delaying the frequency ramp by approximately $5 \mathrm{~ns}$ to wait for the wavelength to settle to its new value. If the stitching errors minimized following the processes presented, repetition rates greater than 50 to $75 \mathrm{kHz}$ or more could be realized. At these rates, a $500 \times 500 \times 500$ pixel image could be produced in less than 4 seconds according to Equation (3.1). 


\section{5 - OCT Implications of Stitching Errors}

Analyzing the entire L-band power leveled linear frequency ramp, saved by the oscilloscope, by The Fast Fourier Transform (FFT) analysis allows a simple method to determine the impact from the stitching errors. A pure sinusoid's FFT, is a delta function at the sinusoid's frequency. The closer the FFT of the sweep data is to a delta function, the more linear the frequency ramp. Figure (7.21) shows that adding two linear ramps together (one delayed in time) will result in a pure sinusoid with frequency $\Delta \mathrm{f}$. If the linear frequency ramp has any defects, widening of the peak in the frequency domain will occur. An ideal sinusoid is a delta function in the frequency domain.

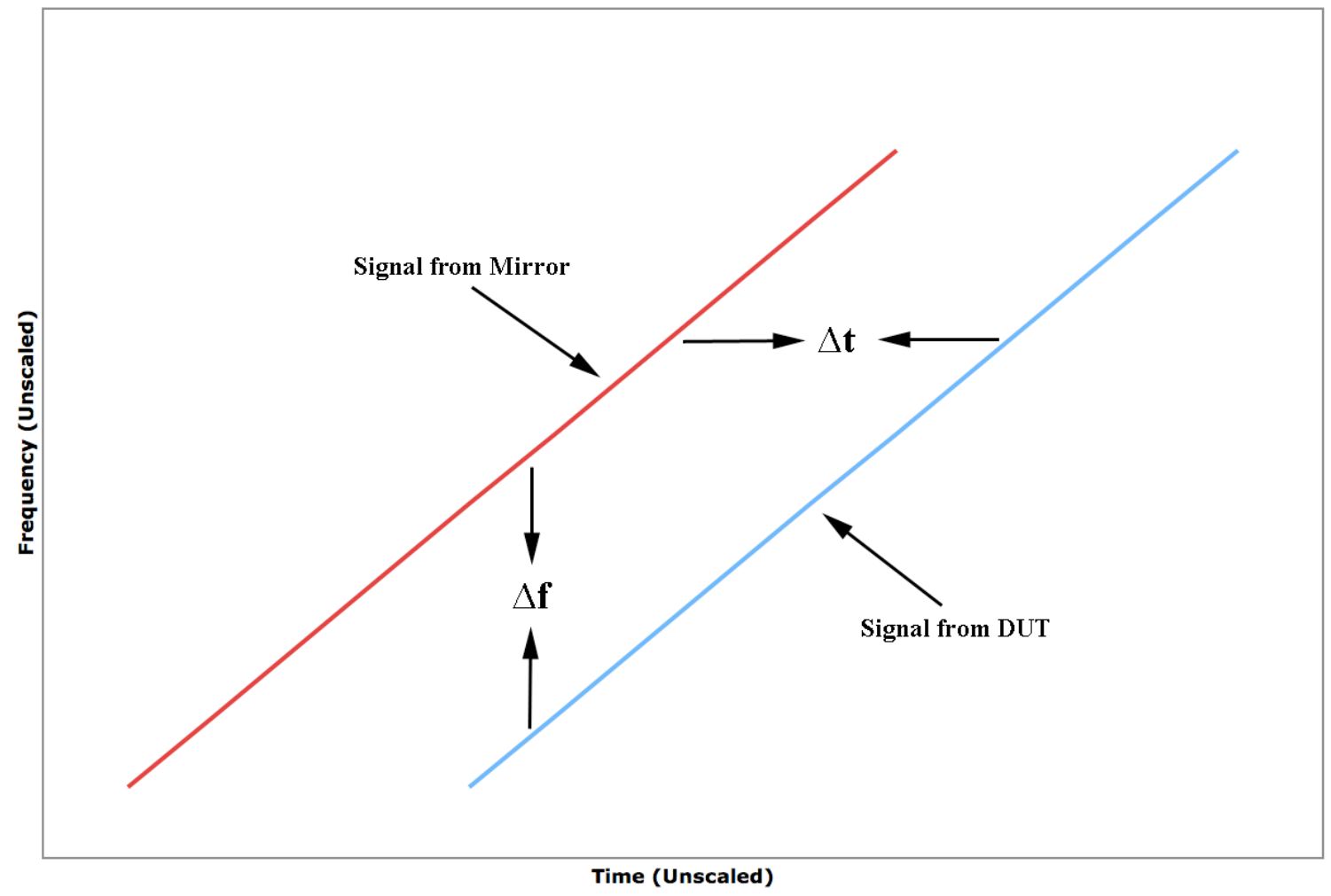

Figure 7.21: Combined signals from the stationary mirror and the DUT. $\Delta t$ is the time difference between the path lengths $L 1$ and $L 2 . \Delta f$ is the beat frequency between the two signals, which also gives the time delay of the DUT signal. Any defects in the linearity, will give multiple $\Delta$ f and cause widening of the peak in the frequency domain, which also causes decreased time resolution. 
Figure (7.22) shows the FFT analysis of three different sweeps. The first (top trace) is the L-band power leveled frequency ramp, the second (middle trace) is the Lband power leveled frequency ramp with the stitches manually "fixed" in Excel after downloading from the oscilloscope, and the third (lowest trace) is a pure sinusoid generated in Excel with the same frequency, amplitude, and quantization. The HP 54501A Digitizing Oscilloscope only uses a small number of bits, 15, for storing the trace. This results in the oscilloscope quantizing its waveform in data represented in signed values from 0 to 32,640, which induces a large source of broadband noise. Figure (7.7) demonstrated the quantization error present in the digitization process. The lowest trace is necessary since the quantization introduces a large source of noise in the FFT analysis. Therefore the lowest trace is the lowest we can expect the top or middle traces to get with no stitching errors. 


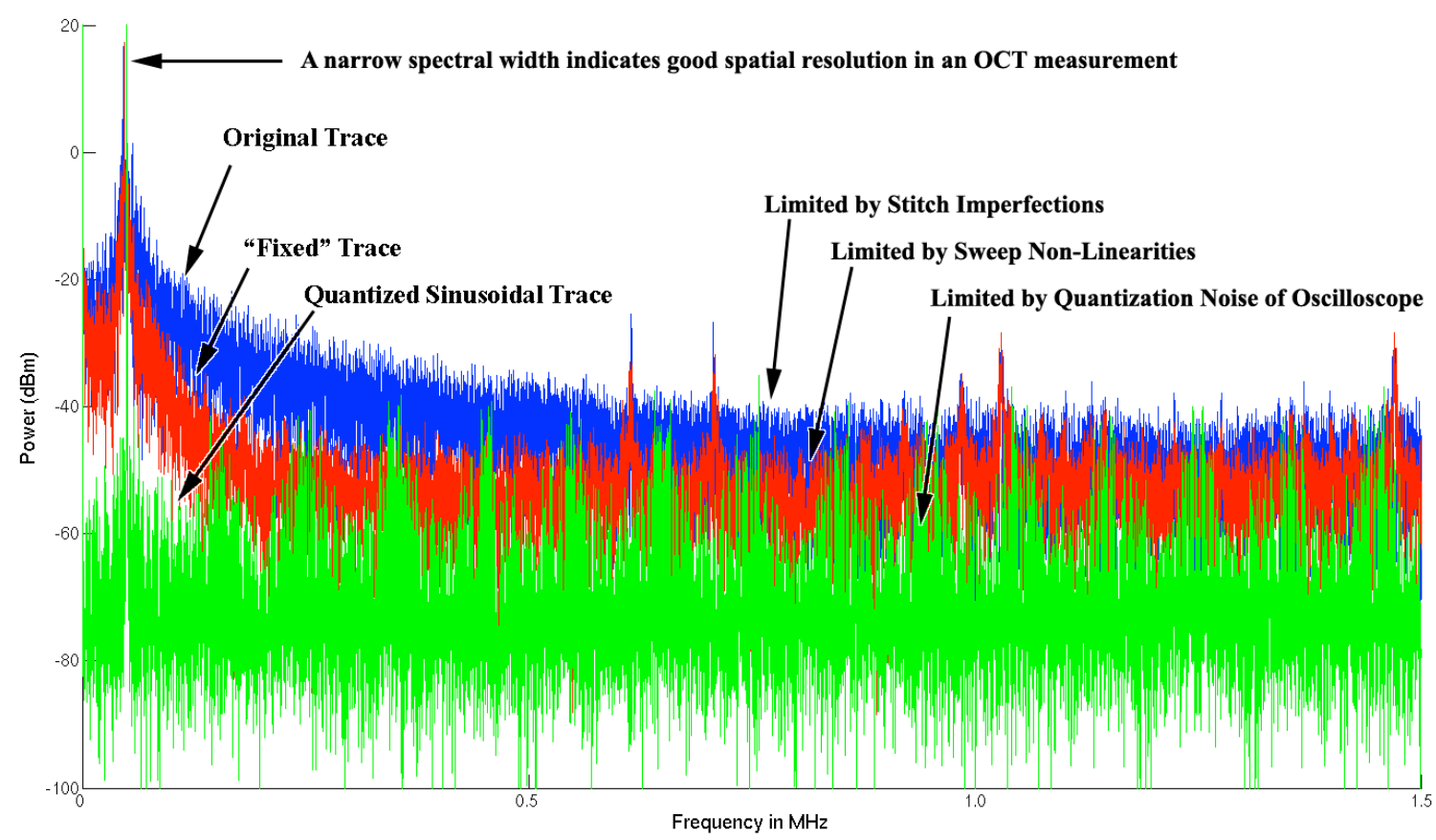

Figure 7.22: Original trace (Top), "fixed" trace (middle), and quantized sinusoid trace (bottom) using FFT analysis. The original trace has the widest peak spectral width, as well as broadband noise, and is limited by the stitching errors of the trace. The "fixed" trace has a much narrower peak, as well as much lower broadband noise, and is limited mostly by small variations from a linear frequency ramp. The quantized sinusoid has extremely narrow peak spectral width, but has a more comparable broadband noise as the quantized trace and is limited by quantization noise.

One can see in Figure (7.22) that fixing the stitches can gain as much as $20 \mathrm{~dB}$ of attenuation over the original data. The $-3 \mathrm{~dB}$ bandwidth of the traces also decreases if the stitches are fixed. Table (7.3) gives the $-3 \mathrm{~dB}$ bandwidth data for the three traces in Figure (7.22).

\begin{tabular}{|c|c|c|c|}
\hline Trace & Original (Blue) & Fixed (Red) & Sinusoid (Green) \\
\hline -3 dB Bandwidth & $\mathbf{4 8 2 . 4 ~ H z}$ & $273.3 \mathrm{~Hz}$ & $\mathbf{2 9 . 0 ~ H z}$ \\
\hline
\end{tabular}

Table 7.3: -3 dB bandwidths for the three traces shown in Figure (7.21).

Performing OCT tests on the various laser ramps created gives insight into the characteristics of the laser output. An Agilent 8504B Michelson Interferometer provides a tunable delay for the OCT test. Figure (7.23) shows the OCT test setup used in this experiment. The laser output is split into two paths by the $8504 \mathrm{~B}$. One path is delayed a set amount by a amount of time in the 8504B. The two outputs are then combined again, 
converted into an electrical signal by a photodiode, amplified, and then sent to an Agilent 8568B Electrical Spectrum Analyzer. The spectrum analyzer displays the spectral content of the combined signal and allows the viewing of the beat signal.

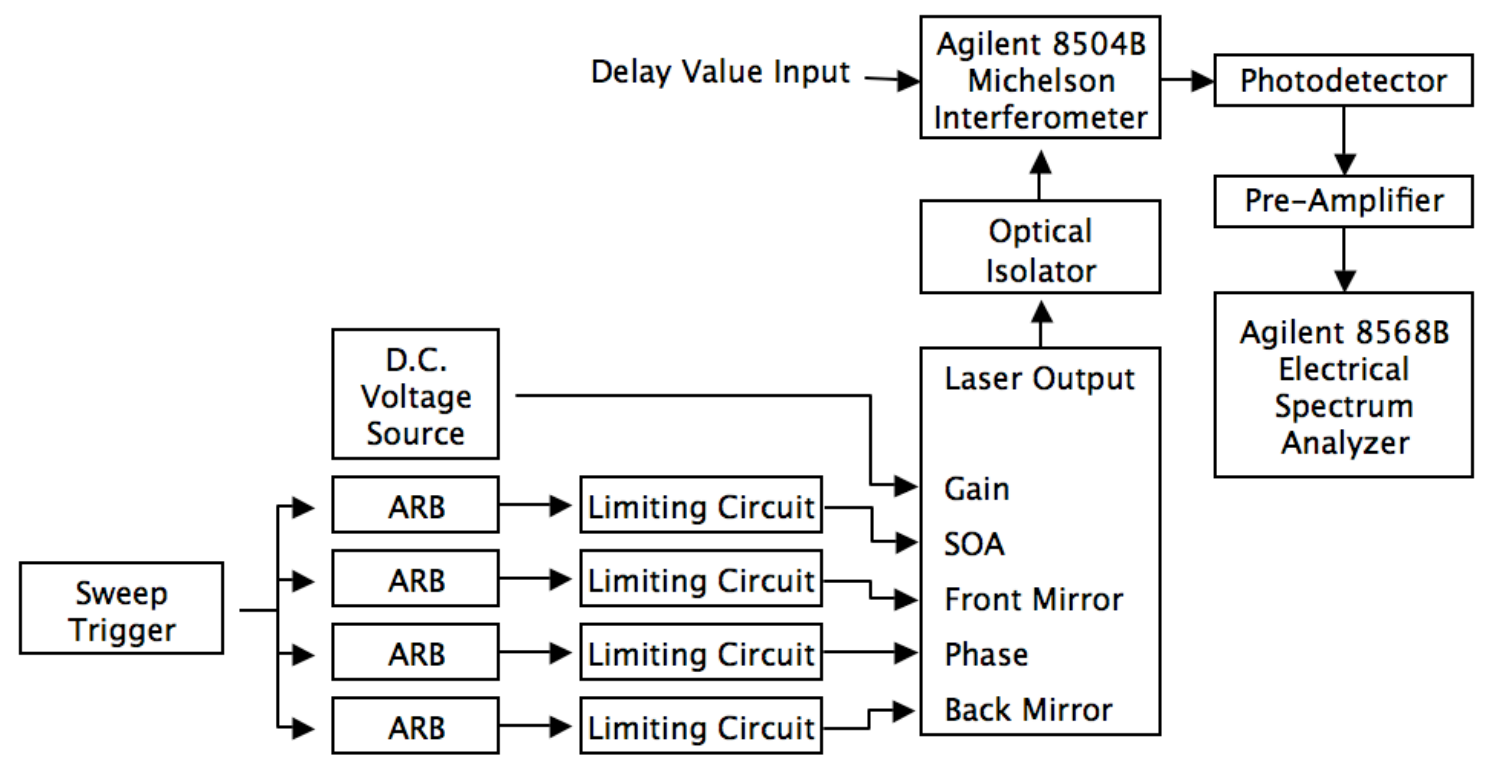

Figure 7.23: Test setup used for OCT testing of the L-band and C-band frequency ramps.

Figure (7.24) shows the result of using a $3 \mathrm{~mm}$ delay on the $8504 \mathrm{~B}$ with the output from the L-band power leveled linear frequency ramp. The spike of $-19.38 \mathrm{dBm}$ at $11.7 \mathrm{kHz}$ results from the beat frequency between the original laser output and the 3 $\mathrm{mm}$ delayed version. The spike at D.C. comes from the local oscillator signal in the spectrum analyzer getting converted down to $0 \mathrm{~Hz}$. 


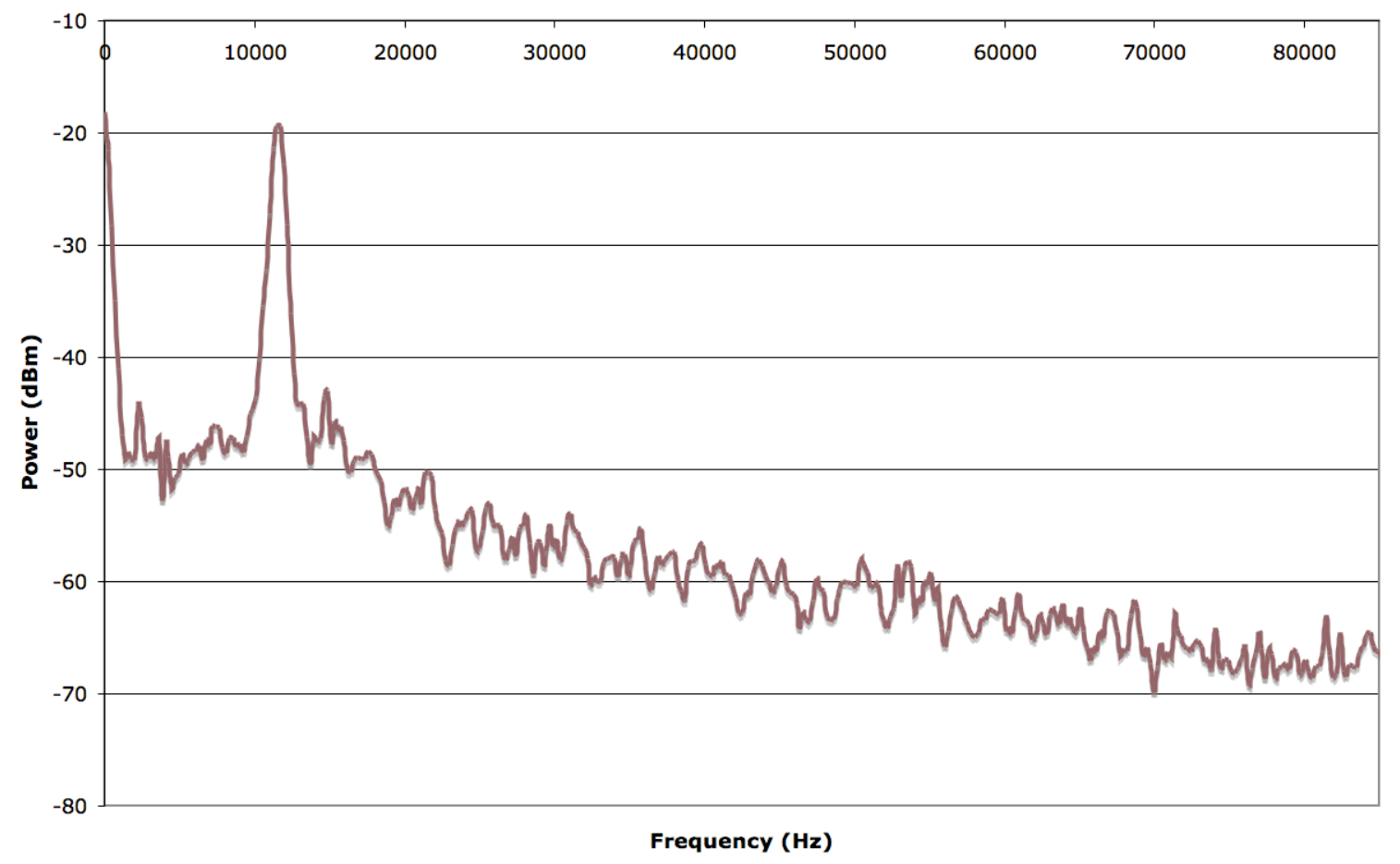

Figure 7.24: Spectrum analyzer output resulting from a $3 \mathrm{~mm}$ delay setting on the $8504 \mathrm{~B}$.

The measurement shown in Figure (7.24) needs to be repeated for values from 0 $\mathrm{mm}$ to $20 \mathrm{~mm}$ delay. Figure (7.25) displays all the measurements of the L-band power leveled linear frequency ramp with various delay lengths overlaid together. 


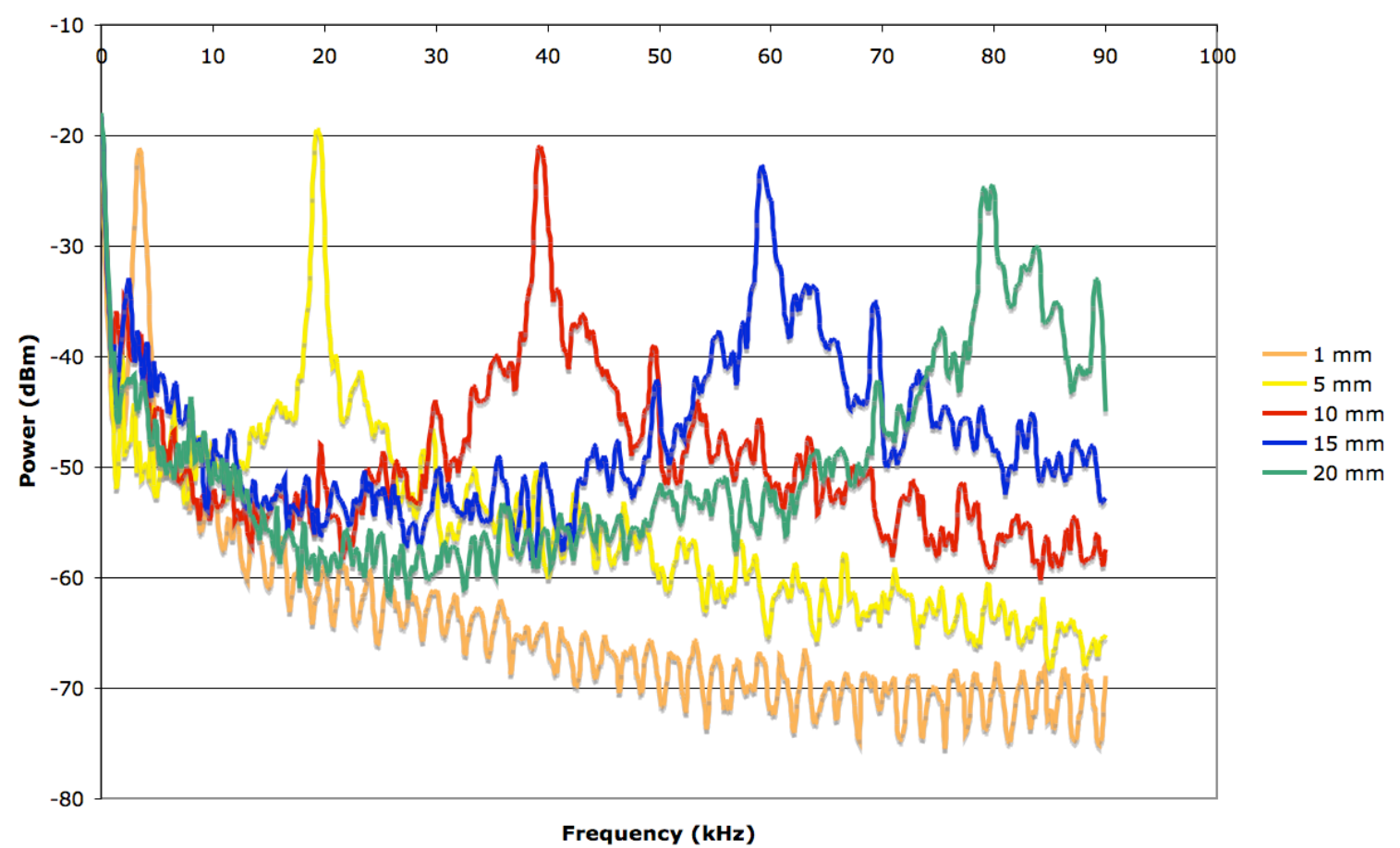

Figure 7.25: Combined OCT measurements showing the beat signals for selected delays from $0 \mathrm{~mm}$ to $20 \mathrm{~mm}$. Note the broadening of the peak signal as the delay increases. As the delay increases in an OCT test, the non-linearities effect the measurement more and more.

One drawback from the measurement shown in Figure (7.25) depends both on the linearity of the frequency ramp, but also on the laser linewidth. The first data extracted from the measurements shown in Figure (7.25) consists of the maximum power level of the peak in each separate delay measurement. The peak power level tends to drop as the delay increases. The more linear the frequency ramp is, the less the peak power level drops as the delay increases. Figure (7.26) compares this data for L-band laser ramps at $100 \mathrm{~Hz}$ repetition rate, Figure (7.27) shows the same information but for the C-band laser at $100 \mathrm{~Hz}$ repetition rate, Figure (7.28) has the L-band $10 \mathrm{kHz}$ repetition rate data, and Figure (7.29) illustrates the $\mathrm{C}$-band $10 \mathrm{kHz}$ repetition rate data. 
100 Hz L-band OCT Peak Power Data

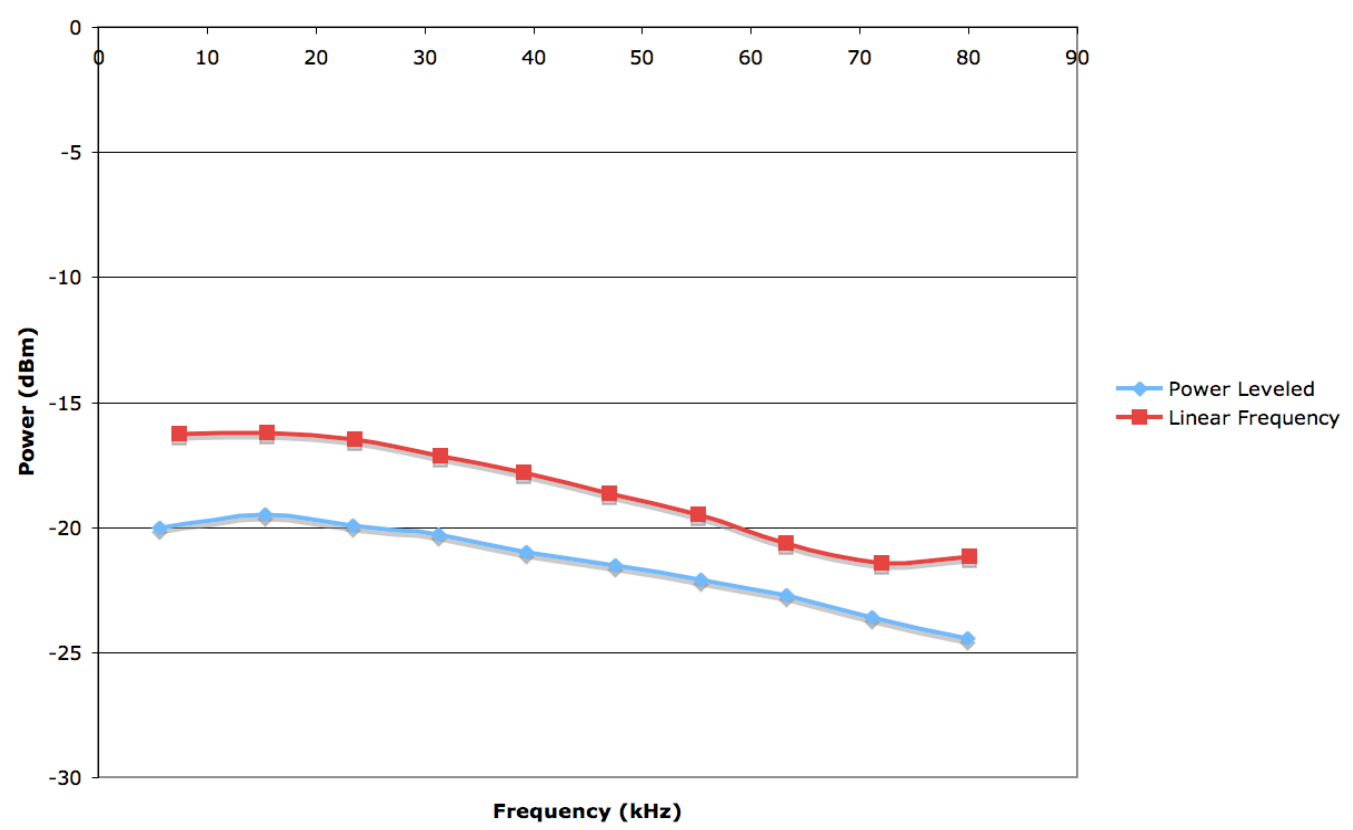

Figure 7.26: OCT peak power data for $L$-band laser at $100 \mathrm{~Hz}$ repetition rate for delays of $2,4,6,8$, $10,12,14,16,18$, and $20 \mathrm{~mm}$. The "Blue" trace is the power leveled linear frequency ramp. The "Red" trace is the non-power leveled linear frequency ramp.

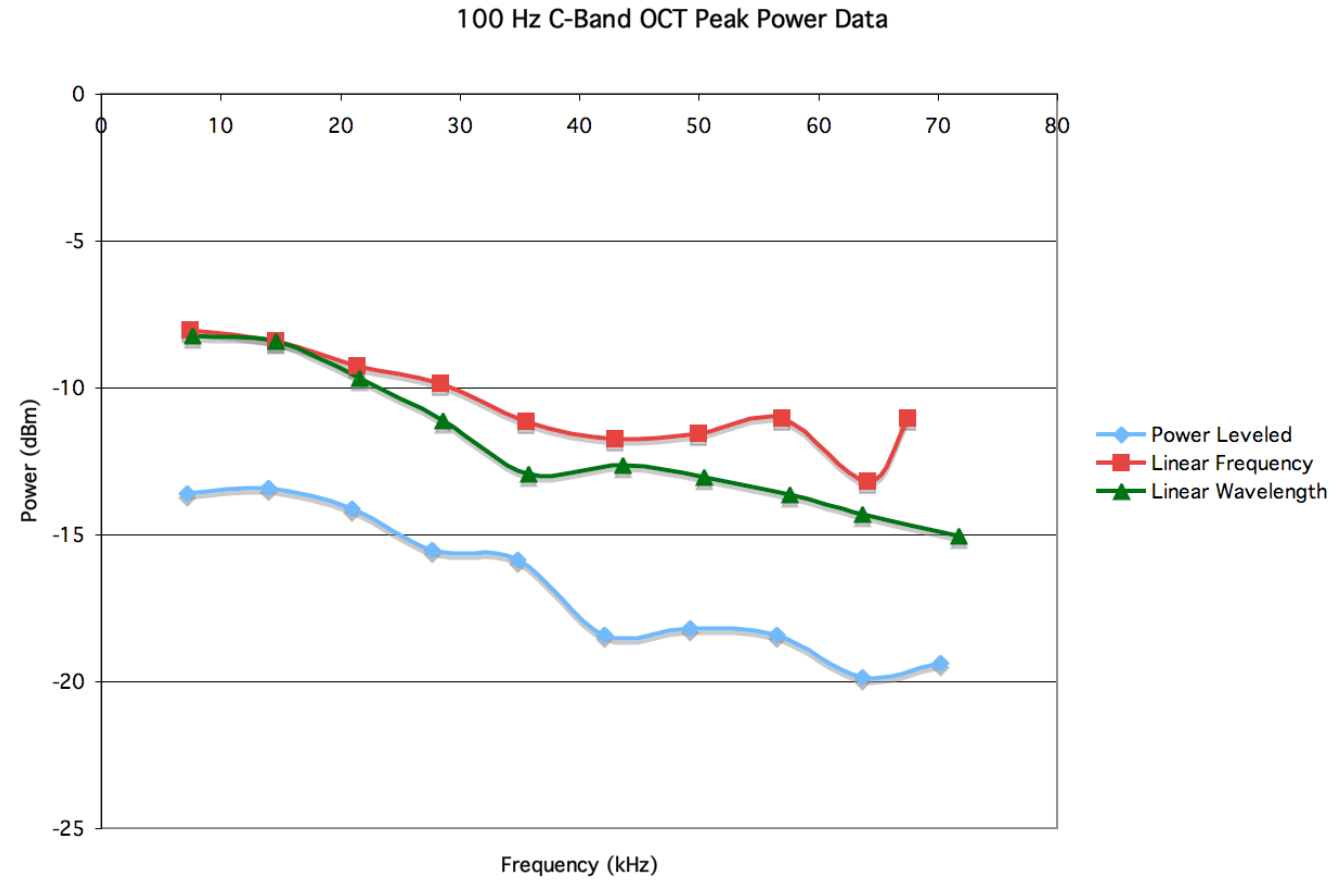

Figure 7.27: OCT peak power data for $\mathrm{C}$-band laser at $100 \mathrm{~Hz}$ repetition rate for delays of 2, 4, 6, 8, $10,12,14,16,18$, and $20 \mathrm{~mm}$. The "Blue" trace is the power leveled linear frequency ramp. The "Red" trace is the non-power leveled linear frequency ramp. Also included is a linear wavelength ramp which peak power values drop off much more quickly than the linear frequency ramps. 
10 kHz L-Band OCT Peak Power Data

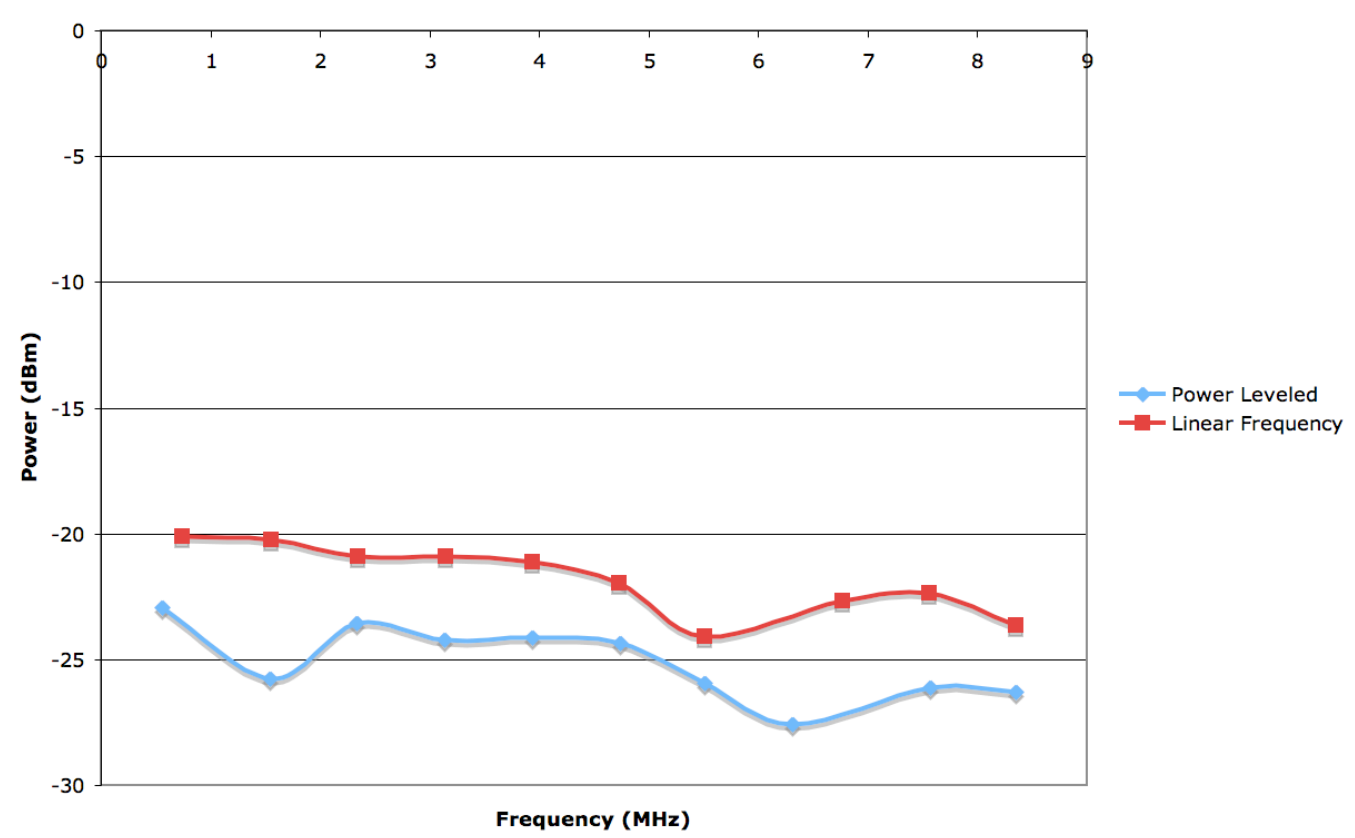

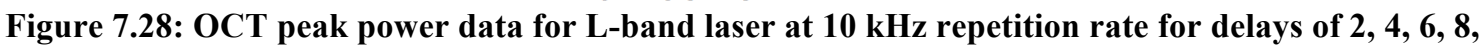
$10,12,14,16,18$, and $20 \mathrm{~mm}$. The "Blue" trace is the power leveled linear frequency ramp. The "Red" trace is the non-power leveled linear frequency ramp.

10 kHz C-Band OCT Peak Power Data

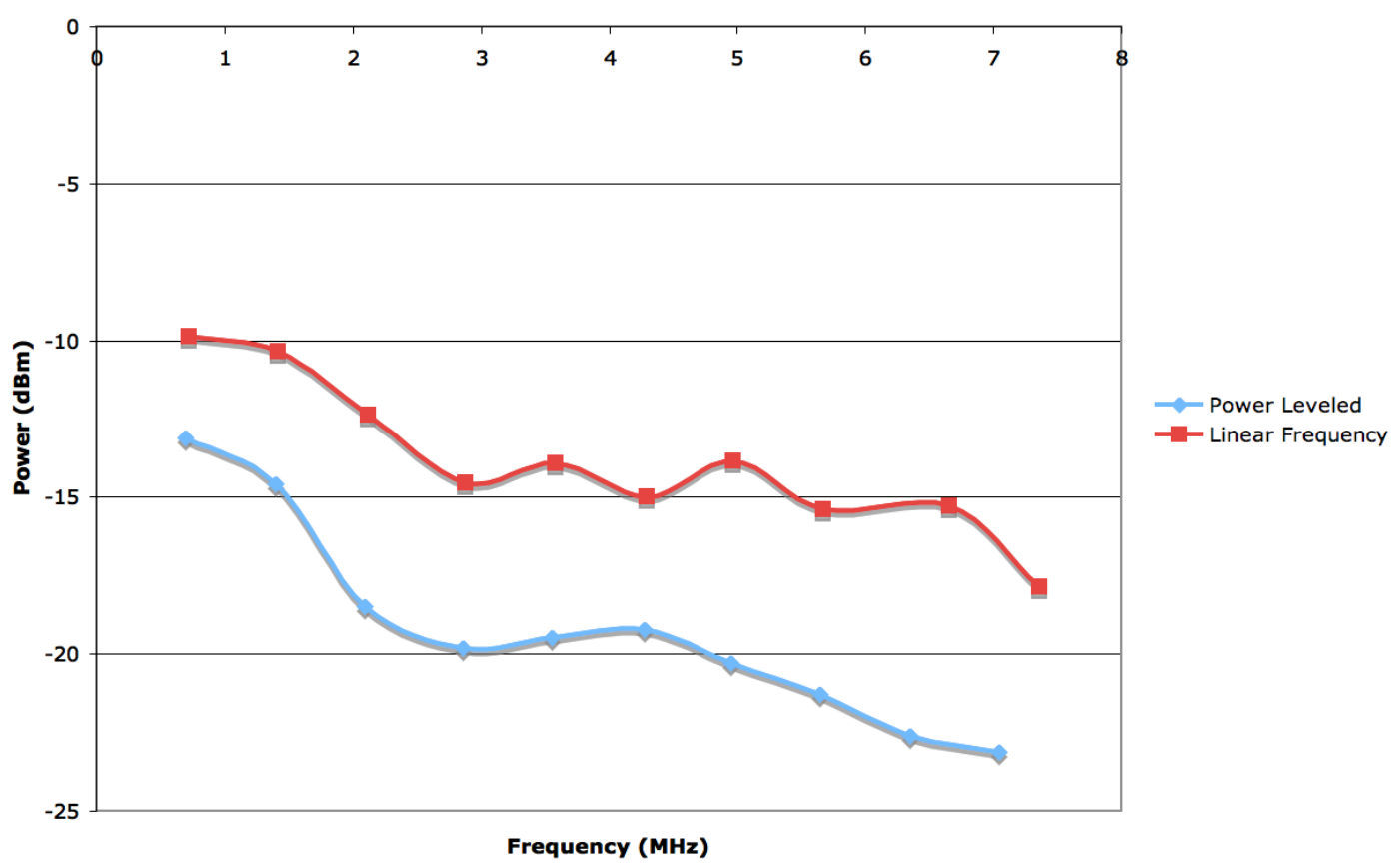

Figure 7.29: OCT peak power data for C-band laser at $10 \mathrm{kHz}$ repetition rate for delays of 2, 4, 6, 8, $10,12,14,16,18$, and $20 \mathrm{~mm}$. The "Blue" trace is the power leveled linear frequency ramp. The "Red" trace is the non-power leveled linear frequency ramp. 
From Figure (7.27) one can see that the linear frequency ramp is more linear than the linear wavelength ramp, as expected from Section 4.3. Figure (7.26), Figure (7.27), Figure (7.28), and Figure (7.29) also show that leveling the laser output power versus frequency, as in Chapter 6, does not have any notable effect on the linearity of the frequency ramp. Power leveling does not have a noticeable effect on the linearity because the main source of non-linearity of the frequency ramp is due to the stitching errors as shown in Figure (7.22).

The second set of data extracted from the OCT test measurements consists of the $-3 \mathrm{~dB}$ width of the beat signal. As the delay increases, in the OCT test, the spectral width of the pulse tends to increase. This can limit the resolution of the OCT measurement. Table (7.4) and Table (7.5) compile this information for the L-band and C-band lasers respectively.

\begin{tabular}{|c|c|c|c|c|}
\hline \multicolumn{4}{|c|}{ L-band OCT Pulse -3 dB Width Data } \\
\hline Rate & \multicolumn{2}{|c|}{$100 \mathrm{~Hz}$} & \multicolumn{2}{c|}{$10 \mathrm{kHz}$} \\
\hline Type & Power Leveled & Linear Frequency & Power Leveled & Linear Frequency \\
\hline $1.5 \mathrm{~mm}$ & $1.131 \mathrm{kHz}$ & $1.085 \mathrm{kHz}$ & $110.3 \mathrm{kHz}$ & $108.5 \mathrm{kHz}$ \\
\hline $10 \mathrm{~mm}$ & $7.937 \mathrm{kHz}$ & $7.914 \mathrm{kHz}$ & $447.1 \mathrm{kHz}$ & $789.3 \mathrm{kHz}$ \\
\hline $20 \mathrm{~mm}$ & $159.1 \mathrm{kHz}$ & $159.3 \mathrm{kHz}$ & $16.70 \mathrm{MHz}$ & $16.70 \mathrm{MHz}$ \\
\hline
\end{tabular}

Table 7.4: Beat signal spectral width data for the $\mathrm{L}$-band laser at $100 \mathrm{~Hz}$ and $10 \mathrm{kHz}$ repetition rates.

\begin{tabular}{|c|c|c|c|c|}
\hline \multicolumn{5}{|c|}{ C-band OCT Pulse -3 dB Width Data } \\
\hline Rate & \multicolumn{2}{|c|}{$100 \mathrm{~Hz}$} & \multicolumn{2}{c|}{$10 \mathrm{kHz}$} \\
\hline Type & Power Leveled & Linear Frequency & Power Leveled & Linear Frequency \\
\hline $1.5 \mathrm{~mm}$ & $1.062 \mathrm{kHz}$ & $1.176 \mathrm{kHz}$ & $110.8 \mathrm{kHz}$ & $108.5 \mathrm{kHz}$ \\
\hline $10 \mathrm{~mm}$ & $7.055 \mathrm{kHz}$ & $7.372 \mathrm{kHz}$ & $719.1 \mathrm{kHz}$ & $714.6 \mathrm{kHz}$ \\
\hline $20 \mathrm{~mm}$ & $157.0 \mathrm{kHz}$ & $135.0 \mathrm{kHz}$ & $14.09 \mathrm{MHz}$ & $14.72 \mathrm{MHz}$ \\
\hline
\end{tabular}

Table 7.5: Beat signal spectral width data for the C-band laser at $100 \mathrm{~Hz}$ and $10 \mathrm{kHz}$ repetition rates.

In this chapter sources of, fixes for, stability of, physical limitations of, and OCT implications of stitching errors were outlined. Future work needs to be done in fixing the 
every stitching error present in the linear frequency ramps since detailed analysis of the linear frequency ramps can occur until the stitching errors are minimized. 


\section{Chapter 8 - Conclusions and Future Work}

This thesis presents power leveled linear frequency ramps that are a useful for OCT measurements. These linear frequency ramps have been shown to cover the Lband, C-band, and a concatenated $\mathrm{L}$ and $\mathrm{C}$ band and we created by modulating four bias ports per laser with tuning waveforms. Three ports of the SG-DBR were used as a frequency control, while the fourth port controlled the output power of the laser.

First, in Chapter 4 L-band and C-band linear frequency ramps were created by adapting techniques from previous research. The main contribution presented in Chapter 4 was the interpolation program which spaced front mirror, back mirror, and phase ARB waveform data points linearly in frequency. Additional research includes creating an automated program that could perform the three-dimensional characterization of the SGDBR laser would save hours in creating a linear frequency ramp. Optimizing the 0.3 nanometer primary side modes of the laser output is the primary issue in creating such a program

Secondly in Chapter 5, the output laser power ripple versus frequency was reduced by modulating the current into the SOA. Output power of the laser versus SOA ARB voltage was characterized. This allowed for a fourth waveform inputted to the SOA section to be used for power leveling the linear frequency ramp. Future research includes refining the power leveling by doing additional leveling on the power leveled linear frequency ramp to flatten the output power versus frequency much more.

Thirdly in Chapter 6, the L-band and C-band linear frequency ramps were concatenated into a single linear L/C-band linear frequency ramp. Concatenating two 
laser ramps into a single ramp allowed for increasing the spectral width of the OCT source, which increases the time resolution of the OCT measurement.

Fourth, Chapter 7 analyzed stitching errors that occur in the linear frequency ramp which are the primary source of error. The stitching errors were shown to repeat frequency coverage in the linear frequency ramp and a method of deleting the extra frequencies from the waveforms was outlined. Further work includes fixing each of the 50 stitching errors in the L-band and C-band linear frequency ramps so accurate measurements of the lasers capabilities can occur.

Fifth, Chapter 7 then showed that at faster rates there was a dead time associated with the $\mathrm{ARB}$ or even the laser sections themselves. A method of delaying the frequency sweep until the each component had settled was shown.

Sixth, Chapter 7 also showed some of the effects of residual stitching errors. FFT analysis was used to calculate the amount of noise was introduced into the measurement from different types of non-linearities.

Seventh, Chapter 7 finally investigated the fundamental switching times of the SG-DBR laser. While the switching times were found to be less than $1 \mathrm{~ns}$, this was a small signal test and large signals in the SG-DBR laser will cause much longer switching times of approximately $5 \mathrm{~ns}$. Further research includes new smaller, more compact, and faster control circuit which could drive the four ports with waveforms with less than $5 \mathrm{~ns}$ rise and fall times. The current system using Agilent 33220A Arbitrary Waveform Generators with $50 \mathrm{~ns}$ rise and fall times are far too slow for repetition rates of $10 \mathrm{kHz}$ and above. Also the large test setup means that there is little guarantee that each ARB sends the waveform into its respective port and the exact same time, since the ARB has 
approximately $15 \mathrm{~ns}$ of trigger jitter and the cables used to connect the ARB will delay each path slightly differently.

In conclusion, this thesis presents the SG-DBR laser as a practical solution to the need for high repetition rate and wide spectral range sources for Swept Frequency OCT. Removing each stitching error in the linear frequency ramp of the L-band and C-band lasers require additional research. The capability of concatenating arbitrarily many overlapping wavelength SG-DBR lasers to create a single linear frequency ramp with high repetition rates is advantageous for increasing time resolution in OCT measurements. 


\section{References}

[1] Dennis Derickson, Mike Bernacil, Andrew DeKelaita, Ben Maher, Shane O'Connor, Matthew N. Sysak, and Leif Johanssen, "SGDBR single-chip wavelength tunable lasers for swept source OCT," Electrical Engineering Department, California Polytechnic State University, San Luis Obispo.

[2] Shane O'Connor, "High Speed Wavelength Tuning of SG-DBR Lasers for Light Detection and Ranging and Optical Coherence Tomography," Electrical Engineering Department, California Polytechnic State University, San Luis Obispo. June 2008.

[3] JDS Uniphase, “JDSU Widely Tunable Laser Diode Module,” Customer Interface Document. April 2008.

[4] Govind P. Agrawal, Fiber-Optic Communication Systems, New York, New York: John Wiley \& Sons, Inc. 2002.

[5] Gert Scarlet, Geert Morthier, and Roel Baets, "Control of Widely Tunable SSGDBR Lasers for Dense Wavelength Division Multiplexing," Journal of Lightwave Technology, vol. 18, pp. 1128-1138, 8 Aug. 2000.

[6] P. R. Herczfeld, A. J. C. Vieira and Y. Li, "Chirped LIDAR-RADAR for Medical Diagnostics," Center for Microwave-Lightwave Engineering, Drexel University.

[7] “Optical Coherence Tomography Protocol," Fundus Photograph Reading Center. Department of Ophthalmology and Visual Sciences University of Wisconsin Madison, 2001, 25 March 2008, $<$ http://eyephoto.ophth.wisc.edu/ResearchAreas/oct/oct1.htm>.

[8] Thorlabs, “Optical Coherence Tomography," (White Paper, Thorlabs).

[9] Dennis Derickson, Fiber Optic Test and Measurement, Upper Saddle River, New Jersey: Prentice Hall, 1998.

[10] M. Musa and S. Salous, "Ambiguity elimination in HF FMCW radar systems," Department of Electrical Engineering \& Electronics, University of Manchester Institute of Science \& Technology, 12 Jan. 2000.

[11] John E. Simsarian, Michael C. Larson, Henry E. Garrett, Hong Xu, and Timothy A. Strand, "Less Than 5-ns Wavelength Switching With an SG-DBR Laser," IEEE Photonics Technology Letters, vol.18, pp. 565-567, 15 Feb. 2006.

[12] David K. Cheng, Field and Wave Electromagnetics, New York, New York: Addison-Wesley. 1992. 


\section{Appendix}

SG-DBR Pin-out and Operating Currents

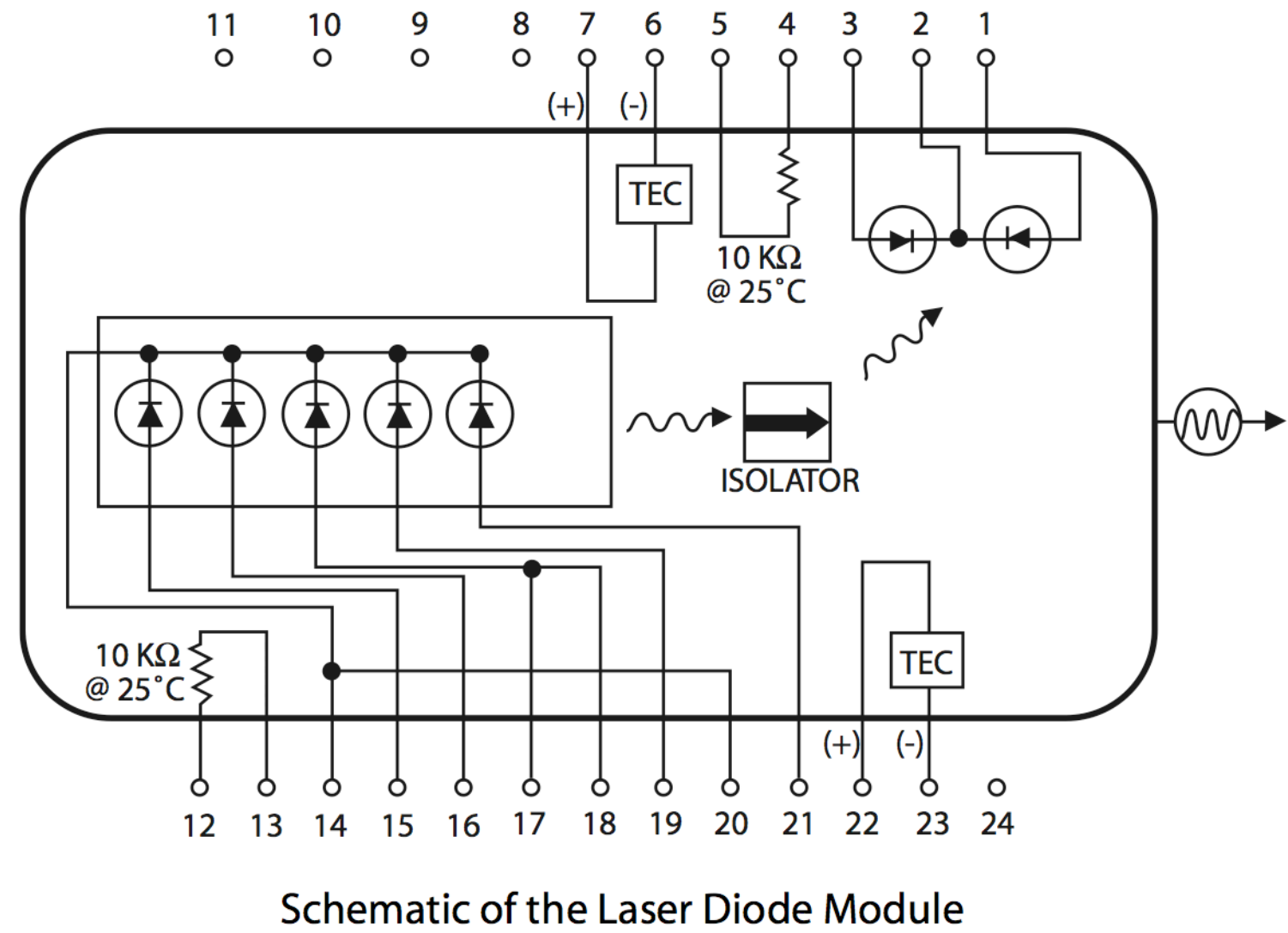




\begin{tabular}{|c|c|c|}
\hline Pin Number & Description & Maximum Safe Operating Current \\
\hline 1 & Etalon Photodiode Anode (-) & \\
\hline 2 & Photodiode Common Cathode (+) & \\
\hline 3 & Reference Photodiode Anode (-) & \\
\hline 4 & Locker Thermistor & \multirow{2}{*}{$100 \mu \mathrm{A}$} \\
\hline 5 & Locker Thermistor & \\
\hline 6 & Locker TEC (-) & \\
\hline 7 & Locker TEC (+) & \\
\hline 8 & $\mathrm{~N} / \mathrm{C}$ & \\
\hline 9 & $\mathrm{~N} / \mathrm{C}$ & \\
\hline 10 & $\mathrm{~N} / \mathrm{C}$ & \\
\hline 11 & $\mathrm{~N} / \mathrm{C}$ & \\
\hline 12 & Laser Thermistor & \multirow{2}{*}{$100 \mu \mathrm{A}$} \\
\hline 13 & Laser Thermistor & \\
\hline 14 & Laser Cathode (-) & \\
\hline 15 & Back Mirror Anode (+) & $60 \mathrm{~mA}$ \\
\hline 16 & Phase Anode (+) & $20 \mathrm{~mA}$ \\
\hline 17 & Gain Anode (+) & \multirow{2}{*}{$150 \mathrm{~mA}$} \\
\hline 18 & Gain Anode (+) & \\
\hline 19 & Front Mirror Anode (+) & $35 \mathrm{~mA}$ \\
\hline 20 & Laser Cathode (-) & \\
\hline 21 & SOA Anode $(+)$ & $195 \mathrm{~mA}$ \\
\hline 22 & Laser TEC (+) & \\
\hline 23 & Laser TEC (-) & \\
\hline 24 & $\mathrm{~N} / \mathrm{C}$ & \\
\hline
\end{tabular}

